REPRESENTATION THEORY

An Electronic Journal of the American Mathematical Society

Volume 3, Pages 281-353 (September 28, 1999)

S $1088-4165(99) 00083-7$

\title{
BASES IN EQUIVARIANT $K$-THEORY. II
}

\author{
G. LUSZTIG
}

\begin{abstract}
In this paper we establish a connection between the "bases" in Bases in equivariant $K$-theory, Represent. Theory 2 (1999), 298-369 and the periodic $W$-graphs introduced in Periodic $W$-graphs, Represent. Theory 1 (1997), 207-279.
\end{abstract}

\section{INTRODUCTION}

0.1. Let $e$ be a nilpotent element in a simple Lie algebra over C. In [L5] we have introduced (under some hypothesis on $e$ ) a candidate for a signed basis of the equivariant $K$-theory of the variety $\mathcal{B}_{e}$ of Borel subalgebras that contain $e$, and we have speculated on its possible connections with the unrestricted representations of the corresponding Lie algebra in characteristic $p$. (For an updated and revised form of these speculations see [L7].) The main purpose of this paper is to establish a connection between these $K$-theory "bases" and the construction of periodic $W$ graphs in [L4]. (As mentioned in [L5, 0.4], the desire to explain those periodic $W$-graphs in $K$-theoretic terms was one of the motivations of [L5].)

The paper is organized as follows.

In Section 1 we establish the freeness of the equivariant $K$-groups used in this paper. This freeness property was conjectured in [L5, 11.7]; a key ingredient in the proof is provided by the results in [DLP].

In Section 2 we establish a duality between the equivariant $K$-theory of $\mathcal{B}_{e}$ and that of the corresponding Slodowy variety $\Lambda_{e}$. Here we must in general extend scalars to rational numbers (due probably to the author's ignorance) but in the case relevant to periodic $W$-graphs the duality works over $\mathbf{Z}$.

In Section 3 we introduce (following [L7]) an element $\nabla_{e}$ in the representation ring and prove a key property of it (Theorem 3.5) which was stated without proof in [L7]. The element $\nabla_{e}$ is one of the ingredients in the conjecture [L7] on unrestricted representations.

In Section 4 we prove that the structure sheaf on $\Lambda_{e}$ is an antispherical vector for the Hecke algebra action.

In Section 5 we extend the definition $[\mathrm{L} 5,12.18]$ of $\mathbf{B}_{\mathcal{B}_{e}}^{ \pm}, \mathbf{B}_{\Lambda_{e}}^{ \pm}$to a general $e$.

In Section 6 we assume that we are given a Levi of a parabolic subalgebra which contains $e$ and we introduce two varieties $\mathfrak{Z}, \tilde{\mathfrak{Z}}$ such that $\mathcal{B}_{e} \subset \tilde{\mathfrak{Z}} \subset \mathfrak{Z} \subset \Lambda_{e}$. In Section 7 we show that the equivariant $K$-groups of these two varieties may be regarded as induced modules of the affine Hecke algebras. (See Theorem 7.11.)

Received by the editors March 9, 1999 and, in revised form, April 15, 1999 and August 7, 1999. 1991 Mathematics Subject Classification. Primary 20G99.

Supported by the Ambrose Monnel Foundation and the National Science Foundation.

(C)1999 American Mathematical Society 
This can be viewed as a substantial strengthening of the induction theorem in [KL].

In Section 8 we study the case where $e$ is regular in a Levi of a parabolic subalgebra. In this case we have $\mathfrak{Z}=\tilde{\mathfrak{Z}}$ and we can describe in detail the equivariant $K$-theory of $\mathfrak{Z}$. (In the case where $e=0, \mathfrak{Z}$ is more or less the same as the Steinberg variety of triples; but in the general case, the proof in Section 6 of the fact that $\mathfrak{Z}$ admits a cell decomposition with all cells of the same dimension is rather more difficult than for $e=0$.)

In Section 9 we review some definitions and results of [L4] in a somewhat modified form. (In particular, the polynomials $\pi_{B, A}$ in 9.17 are obtained from the polynomials $\Pi_{A, B}$ in $[\mathrm{L} 4,11.2]$ by applying an order reversing involution of the set of alcoves and by replacing $v$ by $-v$.)

In Section 10 we establish the connection between our $K$-theory "bases" and the constructions of [L4] (in the form of Section 9).

Section 11 contains an inversion formula extending a result in $[\mathrm{L} 4, \S 12]$.

Section 12 contains an extension of [L4, 11.17]. Note that the proof of [L4, 11.17] relied on [L4, 8.9] whose proof, as A. Braverman pointed out to me, contained a gap. By using Theorem 7.11 instead of the induction theorem [KL], that gap is filled and at same time we have a proof of the conjecture in the first line of [L4, 8.13] concerning the poles of an intertwining operator.

In Section 13 we construct an element of $\mathbf{B}_{\mathcal{B}_{e}}^{ \pm}$for $e$ as in Section 8, assumed to be of Richardson type, corresponding to an irreducible component of $\mathcal{B}_{e}$ which is a flag manifold of a smaller group; this element is essentially a square root of the canonical bundle of that component.

In Section 14 we show that the element in Section 13 generates in a suitable sense the equivariant $K$-theory of $\mathcal{B}_{e}$ (if the group is simply connected). This last condition is relaxed in Section 15.

Section 16 contains a number of examples.

Section 17 contains some conjectures. In 17.1 we explain a conjecture relating the $K$-theory "bases" with cells in the affine Weyl group. In 17.2 we restate and extend the conjecture in $[\mathrm{L} 7,2.4]$ which relates the $K$-theory "bases" with unrestricted representations in characteristic $p$ and in 17.3 we state a strengthening of this conjecture in the case where $e$ is as in Section 8 .

I wish to thank J. C. Jantzen and D. A. Vogan for pointing out a number of misprints and inaccuracies in an earlier version of this paper.

0.2. Notation. Let $G$ be a connected, reductive algebraic group. Let $\mathfrak{g}$ be the Lie algebra of $G$ and let $\mathfrak{g}_{\text {nil }}$ be the variety of nilpotent elements in $\mathfrak{g}$. Let $\mathcal{B}$ be the variety of all Borel subalgebras of $\mathfrak{g}$. Let $\nu=\operatorname{dim} \mathcal{B}$. Let $\Lambda^{\prime}=\{(y, \mathfrak{b}) \in \mathfrak{g} \times \mathcal{B} \mid y \in \mathfrak{b}\}$, $\Lambda=\left\{(y, \mathfrak{b}) \in \Lambda^{\prime} \mid y \in \mathfrak{g}_{n i l}\right\}$. The group $G \times \mathbf{C}^{*}$ acts on $\mathcal{B}$ by $(g, \lambda): \mathfrak{b} \mapsto \operatorname{Ad}(g) \mathfrak{b}$ and on $\mathfrak{g}$ by $(g, \lambda): y \mapsto \lambda^{-2} \operatorname{Ad}(g) y$. Then $G \times \mathbf{C}^{*}$ acts on $\Lambda^{\prime}, \Lambda$ by the restriction of the action on $\mathfrak{g} \times \mathcal{B}$ (simultaneous action on both factors).

For any parabolic subalgebra $\mathfrak{p}$ of $\mathfrak{g}$ we denote by $\mathfrak{n}_{\mathfrak{p}}$ the nil-radical of $\mathfrak{p}$. A parabolic subalgebra $\mathfrak{p}$ of $\mathfrak{g}$ is said to be almost minimal if the variety of Borel subalgebras contained in $\mathfrak{p}$ is 1-dimensional. Let $I$ be a finite set indexing the $G$-orbits on the set of almost minimal parabolic subalgebras of $\mathfrak{g}$. A parabolic subalgebra in the $G$-orbit indexed by $i$ is said to have type $i$. If $K$ is a subset of $I$, we say that a parabolic subalgebra $\mathfrak{p}$ of $\mathfrak{g}$ has type $K$, if $K$ is the set of all $i \in I$ such that $\mathfrak{p}$ contains some parabolic subalgebra of type $i$. 
Let $\mathbf{X}$ be the set of isomorphism classes of algebraic $G$-equivariant line bundles on $\mathcal{B}$. We regard $\mathbf{X}$ as a (finitely generated free) abelian group under the operation given by tensor product of line bundles. Let $\mathcal{X}$ be a free abelian group (in additive notation) with a given isomorphism $\mathcal{X} \stackrel{\sim}{\rightarrow} \mathbf{X}$ denoted by $x \mapsto L_{x}$. Thus, $L_{x+x^{\prime}}=$ $L_{x} \otimes L_{x^{\prime}}$ for $x, x^{\prime} \in \mathcal{X}$. For $i \in I$, let $\alpha_{i} \in \mathcal{X}, \check{\alpha}_{i}: \mathcal{X} \rightarrow \mathbf{Z}$ be as in [L5, 7.2]. Let $\mathcal{X}_{\geq}=\sum_{i \in I} \mathbf{N} \alpha_{i} \subset \mathcal{X}, \mathcal{X}_{\leq}=-\mathcal{X}_{\geq}$. Let $\sigma_{i}: \mathcal{X} \rightarrow \mathcal{X}$ be the involution given by $x \mapsto{ }^{\sigma_{i}} x=x-\check{\alpha}_{i}(x) \alpha_{i}$. Let $W$ be the subgroup of $A u t(\mathcal{X})$ generated by the elements $\sigma_{i}, i \in I$. (A Weyl group with simple reflections $\sigma_{i}$ and length function $l: W \rightarrow \mathbf{N}$.)

Let $\mathcal{A}=\mathbf{Z}\left[v, v^{-1}\right]$ where $v$ is an indeterminate. Let $\mathcal{H}$ be the affine Hecke algebra attached to $G$, that is, the associative $\mathcal{A}$-algebra with 1 defined by the generators $\tilde{T}_{w}, w \in W$ and $\theta_{x}, x \in \mathcal{X}$ and the relations:

(a) $\left(\tilde{T}_{\sigma_{i}}+v^{-1}\right)\left(\tilde{T}_{\sigma_{i}}-v\right)=0$;

(b) $\tilde{T}_{w} \tilde{T}_{w^{\prime}}=\tilde{T}_{w w^{\prime}}$ if $l\left(w w^{\prime}\right)=l(w)+l\left(w^{\prime}\right)$;

(c) $\theta_{x} \tilde{T}_{\sigma_{i}}-\tilde{T}_{\sigma_{i}} \theta_{\sigma_{i x}}=\left(v-v^{-1}\right) \theta_{\frac{[x]-\left[\sigma_{i x]}\right]}{1-\left[-\alpha_{i}\right]}}$;

(d) $\theta_{x} \theta_{x^{\prime}}=\theta_{x+x^{\prime}}$

(e) $\theta_{0}=1$.

Here relation (c) has the same meaning as in [L5, 1.19].

In this paper, all algebraic varieties are assumed to be quasiprojective over $\mathbf{C}$, unless otherwise specified.

For a finite set $X$ we denote by $|X|$ the number of elements of $X$.

\section{ERRATA TO [L5]}

2.18, line 2: delete "the equality ${ }^{\sigma_{i}} \rho=\rho-\alpha_{i}$ "

3.8(b): replace $n<n_{0}$ by $n>n_{0}$

p.314, line 4: replace $[x] m,[x] \tilde{m},\left[x^{\prime}\right] m^{\prime},\left[x^{\prime}\right] \tilde{m}^{\prime}$ by ${ }_{\iota} m,{ }_{\iota} \tilde{m}, \iota^{\prime} m^{\prime},{ }_{\iota}{ }^{\prime} \tilde{m}^{\prime}$

3.14(b): replace $\tilde{b}$ by $\tilde{\hat{b}}$

3.14(c): replace $b$ by $\hat{b}$

p.315, line $-2,-1$ : replace $\Pi^{\prime}$ by $\Pi$

p.315, line -1: replace last $=$ by $\epsilon$

p.316, line 3: replace last $=$ by $\in$

p.316, line 7: insert ) after $\mathcal{A}$

$5.2(\mathrm{a})$ : replace, by :

5.17: replace "We apply the identity 5.15 (a) with" by "Let"

5.17: replace "as in the proof of 5.15" by "as in the proof of 5.14"

7.9 , line 5: replace $p_{12}, p_{13}, p_{23}$ by $\pi_{12}, \pi_{23}, \pi_{13}$

7.9 (a): replace $p_{12}, p_{13}, p_{23}$ by $\pi_{12}, \pi_{13}, \pi_{23}$

7.14, line 2: replace $\Lambda^{2}$ by $\Lambda \times \mathcal{B}$

7.16 , line 4 : replace "line bundle" by "vector bundle"

$7.18(\mathrm{a})$ : replace $v$ by $v^{2}$

7.19, last line: replace $\mathbf{Z}_{i}$ by $\bar{Z}_{i}$

$7.23,7.24,7.25$ : replace $\tilde{T}_{i}$ by $\tilde{T}_{\sigma_{i}}$

8.3,8.4: replace $s_{i}$ by $\sigma_{i}$

8.4, line 1,2: replace $h: Z_{w} \rightarrow Z_{\leq w}$ by $h: Z_{\leq w} \rightarrow Z_{w}$

p.337, line 1: delete and replace by "We have"

p.337, line 2: replace $k^{*}$ by $h^{*}$

8.7, line 2: replace $Z_{w}$ by $Z_{w^{\prime}}$ 
8.7(b): replace the last $K$ by $K_{\mathcal{G}}$

8.11: line 4 of proof; replace $v \xi$ by $v \xi^{\prime}$

8.11: last line of proof; replace 10.1 by 8.10

9.7, line 1: replace $D^{\prime}\left(\xi 0\right.$ by $D^{\prime}(\xi)$

p.343, line -3: replace $Z_{0}=\left\{(y, \mathfrak{b}) \in \Lambda \mid \mathfrak{b}^{\prime}=\mathfrak{b}_{0}\right\}$ by $Z_{0}=\left\{(y, \mathfrak{b}) \in \Lambda \mid y \in \mathfrak{n}_{0}\right\}$

10.6, line -5 : the last arrow should have a $\sim$ on top

10.8, 10.10, 10.11, 10.12: replace $g, \tilde{g}$ by $g^{-1}, \tilde{g}^{-1}$

10.10, line 2: replace $L_{x^{\prime}}$ by $L_{x}$

11.3 , line 3: replace $(0, \mathfrak{b})$ by $(e, \mathfrak{b})$

11.3: replace $\lim _{t \rightarrow 0}$ by $\lim _{\lambda \rightarrow 0}$

11.3: replace $\lim _{t \rightarrow \infty}$ by $\lim _{\lambda \rightarrow \infty}$

11.4(a): replace $\mathcal{B}_{e, \mu}^{\mathbf{C}^{*}}$ by $\mathcal{B}_{e, \mu}$

11.4(b): replace $\Lambda_{e, \mu}^{\mathrm{C}^{*}}$ by $\Lambda_{e, \mu}$

11.10: replace $V e c_{\Lambda_{e}}$ by $V e c_{H}\left(\Lambda_{e}\right)$

11.10 (last line): replace $2 \operatorname{dim} \mathfrak{g} / \mathfrak{z}(f)$ by $\operatorname{dim} \mathfrak{g} / \mathfrak{z}(f)$ (twice)

12.1-12.4 replace by: Let $e, f, h, C, \mathfrak{c}, H$ be as in 11.1. We can find an opposition $\varpi$ of $\mathfrak{g}$ such that $\varpi(e)=-e, \varpi(f)=-f, \varpi(h)=h$ and $\varpi=-1$ on $\mathfrak{c}$. We fix such a $\varpi$.

12.9: replace first two sentences by: "Let $d(e)=(1 / 2) \operatorname{dim} \operatorname{Ad}(G) e . "$

12.18 (beginning) add: Let $L$ be the centralizer of $C$ in $G$. In the remainder of this paper we assume that the centralizer of $e, f, g$ in $L$ is equal to the centre of $L$. Then $\varpi$ is uniquely defined by $e, f, h, C$ up to conjugation by $\operatorname{Ad}(c)$ where $c \in C$.

$14.2-14.5$ : replace by the revision in $[\mathrm{L} 7,2.4]$ or by 17.2 in this paper

\section{Contents}

1. Freeness

2. Perfectness of a bilinear pairing

3. The element $\nabla_{e} \in R_{H}$

4. $\mathcal{H}$-module structures

5. The sets $\mathbf{B}_{\mathcal{B}_{e}}^{ \pm}, \mathbf{B}_{\Lambda_{e}}^{ \pm}$

6. The varieties $\mathfrak{Z}, \tilde{\mathfrak{Z}}$

7. $K_{H}(\mathfrak{Z}), K_{H}(\tilde{\mathfrak{Z}})$ as induced $\mathcal{H}$-modules

8. The case where $e$ is regular in $\mathfrak{l}$

9. A review of [L4]

10. Relating $\mathbf{B}_{\mathcal{B}_{e}}^{ \pm}, \mathbf{B}_{\Lambda_{e}}^{ \pm}$to $[\mathrm{L} 4]$

11. Inversion

12. Boundedness of poles

13. Example of an element of $\mathbf{B}_{\mathcal{B}_{e}}^{ \pm}$

14. A case where the $\left(\mathcal{H}, R_{H}\right)$-module $K_{H}\left(\mathcal{B}_{e}\right)$ is cyclic

15. Relaxing the simply connectedness assumption

16. Examples

17. Conjectures

\section{Freeness}

1.1. Let $X$ be an algebraic variety. Let $K(X)$ be the Grothendieck group of the category of coherent sheaves on $X$. Let $H_{u}(X)$ be the degree $u$ integral Borel-Moore homology of $X$. Let $\chi_{X}$ be the Euler characteristic of $X$ in Borel-Moore homology. Let $A_{*}(X)=\bigoplus_{u} A_{u}(X)$ be the Chow group of $X$ (see [F, 1.3]). 
Let $H$ be a torus and assume that $X$ has a given $H$-action. The fixed point set of $H$ on $X$ is denoted by $X^{H}$. Let $\operatorname{Coh}_{H}(X)$ be the abelian category whose objects are the coherent $H$-equivariant sheaves on $X$. Let $\operatorname{Vec}_{H}(X)$ be the full subcategory of $\mathrm{Coh}_{H}(X)$ whose objects are the $H$-equivariant vector bundles on $X$; we identify an object of $\operatorname{Vec}_{H}(X)$ with its sheaf of sections. Let $\mathfrak{O}_{X}$ (or sometimes $\mathbf{C}$ ) be the structure sheaf of $X$ regarded naturally as an object of $\operatorname{Vec}_{H}(X)$. When $H=\{1\}$ we write $\operatorname{Vec}(X)$ instead of $\operatorname{Vec}_{H}(X)$.

Let $K_{H}(X)$ be the Grothendieck group of the category of coherent $H$-equivariant sheaves on $X$. Occasionally, we shall also consider the higher analogues (Quillen, Thomason) $K_{H}^{i}(X)$ of $K_{H}(X)$. In fact we shall only need $K_{H}^{0}(X)=K_{H}(X)$ and $K_{H}^{1}(X)$. Note that $K_{H}^{i}(X)$ are naturally modules over $R_{H}$, the representation ring of $H$. Recall that $R_{H}$ is naturally the group $\operatorname{ring} \mathbf{Z}[\hat{H}]$ where $\hat{H}=\operatorname{Hom}\left(H, \mathbf{C}^{*}\right)$. When $K_{H}(X)$ is a finitely generated free $R_{H}$-module, we shall write $\operatorname{rk} K_{H}(X)$ for its rank (over $R_{H}$ ).

Let $\mathfrak{R}$ be the quotient field of $R_{H}$. For any $R_{H}$-module $M$ we set $M_{\mathfrak{R}}=\mathfrak{R} \otimes_{R_{H}}$ $M$.

1.2. As in [DLP], we say that an algebraic variety $X$ has property $(S)$ if $H_{u}(X)$ is zero for $u$ odd, has no torsion for even $u$ and the canonical map $A_{u}(X) \rightarrow H_{2 u}(X)$ (see $[\mathrm{F}, 19.1])$ is an isomorphism.

Lemma 1.3. If $X$ has property $(S)$, then $K(X)$ is a free abelian group of rank $\chi_{X}$.

It is known that there exists a finite filtration of $K(X)$ whose associated graded $\operatorname{gr} K(X)$ is a homomorphic image of $A_{*}(X)$ (see [F, p. 285]) and that $\mathbf{Q} \otimes \operatorname{gr} K(X) \cong$ $\mathbf{Q} \otimes A_{*}(X)$ (see [F, pp. 354, 355]). By our assumption, $A_{*}(X) \cong \mathbf{Z}^{n}$ for some $n \in \mathbf{N}$. Hence $\operatorname{gr} K(X)$ is either $\mathbf{Z}^{n}$ or $\mathbf{Z}^{n^{\prime}} \oplus$ torsion with $n^{\prime}<n$. The second alternative cannot occur since $\mathbf{Q} \otimes \operatorname{gr} K(X) \cong \mathbf{Q}^{n}$. Thus, $\operatorname{gr} K(X) \cong \mathbf{Z}^{n}$. This forces $K(X) \cong \mathbf{Z}^{n}$. The lemma is proved.

1.4. Assume that $X$ is smooth and projective. Consider the $\mathbf{Z}$-bilinear pairing

(a) $K(X) \times K(X) \rightarrow \mathbf{Z}, \quad E, E^{\prime} \mapsto \pi_{*}\left(E \otimes E^{\prime}\right)$

where $E, E^{\prime} \in \operatorname{Vec}(X)$ and $\pi$ is the canonical map of $X$ to the point.

We say that $X$ has property $\left(S^{\prime}\right)$ if $K(X)$ is a free abelian group of finite rank and the pairing (a) is perfect.

Note that, if $X$ is assumed to have property $(S)$, then the pairing

$$
(\mathbf{Q} \otimes K(X)) \times(\mathbf{Q} \otimes K(X)) \rightarrow \mathbf{Q}
$$

obtained from (a) by extension of scalars is automatically perfect. (This follows easily from the fact that the rational cohomology of $X$ satisfies Poincaré duality and the fact that the Todd class of $X$ is invertible in the rational cohomology algebra of $X$.)

1.5. Let $H$ be a torus. Let $X$ be a smooth projective variety with $H$-action. Consider the $R_{H}$-bilinear pairing

(a) $K_{H}(X) \times K_{H}(X) \rightarrow R_{H}, E, E^{\prime} \mapsto \pi_{*}\left(E \otimes E^{\prime}\right)$

where $E, E^{\prime} \in \operatorname{Vec}_{H}(X)$ and $\pi$ is the canonical map of $X$ to the point. We say that $X$ has property $\left(S_{H}^{\prime}\right)$ if $K_{H}(X)$ is a free $R_{H}$-module of finite rank and the pairing (a) is perfect. 
Lemma 1.6. Assume that $X=X_{1} \cup X_{2} \cup \cdots \cup X_{r}$ is a partition into $H$-stable subsets such that $X_{\leq j}=X_{1} \cup X_{2} \cup \cdots \cup X_{j}$ is closed in $X$ for any $j$, that $X_{j}^{H}$ is closed in $X^{H}$ for any $j$ and that for any $j, K_{H}\left(X_{j}^{H}\right), K_{H}\left(X_{j}\right)$ are free $R_{H}$-modules of the same finite rank $a_{j}$. Then, for any $j, K_{H}\left(X_{\leq j}{ }^{H}\right), K_{H}\left(X_{\leq j}\right)$ are free $R_{H^{-}}$ modules of rank $\sum_{j^{\prime} ; j^{\prime} \leq j} a_{j^{\prime}}$. In particular, $K_{H}\left(X^{H}\right), K_{H}(X)$ are free $R_{H}$-modules of $\operatorname{rank} \sum_{j} a_{j}$.

We argue by induction on $j$. The result is trivial for $j=0$. Assume that $j \geq 1$ and that the result is known for $j-1$. By assumption, $K_{H}\left(X_{j}^{H}\right), K_{H}\left(X_{j}\right)$ are free of rank $a_{j}$ over $R_{H}$. Since $X_{\leq j}^{H}$ is the disjoint union (as variety) of $X_{j^{\prime}}^{H}$ with $j^{\prime} \leq j$, we see that $K_{H}\left(X_{\leq j}^{H}\right)$ is free of rank $\sum_{j^{\prime} ; j^{\prime} \leq j} a_{j^{\prime}}$ over $R_{H}$ and we have a natural exact sequence

$$
0 \rightarrow K_{H}\left(X_{\leq j-1}^{H}\right) \rightarrow K_{H}\left(X_{\leq j}^{H}\right) \rightarrow K_{H}\left(X_{j}^{H}\right) \rightarrow 0
$$

Hence in the commutative diagram

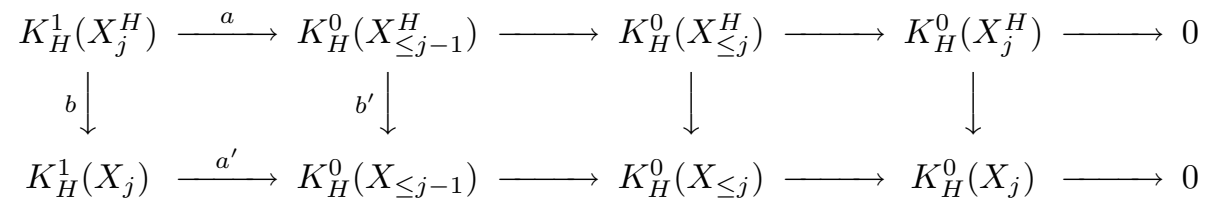

with exact rows (part of the long exact sequence in higher $K$-theory), and with vertical maps given by direct image induced by the inclusion, the boundary map $a$ is 0 . Let $x \in K_{H}^{1}\left(X_{j}\right)$. We show that $a^{\prime} x=0$. By [Th], $b$ is an isomorphism after tensoring by $\mathfrak{R}$. Hence we can find $y \in K_{H}^{1}\left(X_{j}^{H}\right)$ and $f \in R_{H}-\{0\}$ such that $f x=b(y)$. Then $f a^{\prime}(x)=a^{\prime} b(y)=b^{\prime} a(y)=0$ since $a=0$. By the induction hypothesis, $K_{H}^{0}\left(X_{\leq j-1}\right)$ is free over $R_{H}$ hence from $f a^{\prime}(x)=0$ we deduce $a^{\prime}(x)=0$. Thus, we have $a^{\prime}=0$ and we have an exact sequence of $R_{H}$-modules:

$$
0 \rightarrow K_{H}\left(X_{\leq j-1}\right) \rightarrow K_{H}\left(X_{\leq j}\right) \rightarrow K_{H}\left(X_{j}\right) \rightarrow 0
$$

where $K_{H}\left(X_{\leq j-1}\right)$ is free of rank $\sum_{j^{\prime} ; j^{\prime}<j} a_{j^{\prime}}$ and $K_{H}\left(X_{j}\right)$ is free of rank $a_{j}$. It follows that $K_{H}\left(X_{\leq j}\right)$ is free of rank $\sum_{j^{\prime} ; j^{\prime} \leq j} a_{j^{\prime}}$. The lemma is proved.

Lemma 1.7. Let $X$ be a smooth variety with $H$-action. Assume that we are given a homomorphism of algebraic groups $\mathbf{C}^{*} \rightarrow H$ and that, for any connected component $\mu$ of the fixed point set $X^{\mathbf{C}^{*}}$, the $R_{H}$-module $K_{H}(\mu)$ is free of finite rank.

(a) If the induced $\mathbf{C}^{*}$-action contracts (as $\lambda \in \mathbf{C}^{*}$ tends to 0 ) $X$ to a compact subset, then $K_{H}(X)$ is a free $R_{H}$-module of the same rank as $K_{H}\left(X^{\mathbf{C}^{*}}\right)$.

(b) If the induced $\mathbf{C}^{*}$-action contracts (as $\lambda \in \mathbf{C}^{*}$ tends to $\infty$ ) $X$ to a compact subset, then $K_{H}(X)$ is a free $R_{H}$-module of the same rank as $K_{H}\left(X^{\mathbf{C}^{*}}\right)$.

(c) If $X$ is projective and any connected component of $X^{\mathbf{C}^{*}}$ satisfies $S_{H}^{\prime}$, then $X$ satisfies $S_{H}^{\prime}$.

Let $\lambda, x \mapsto \lambda \circ x$ denote the $\mathbf{C}^{*}$-action on $X$ (restriction of the $H$-action). For any connected component $\mu$ of $X^{\mathbf{C}^{*}}$ we denote by $\mu_{+}$, in case (a), (resp. by $\mu_{-}$, in case (b)) the set of all $x \in X$ such that $r_{+}(x)=\lim _{\lambda \rightarrow 0} \lambda \circ x \in \mu$ (resp. $r_{-}(x)=\lim _{\lambda \rightarrow \infty} \lambda \circ x \in \mu$.) It is known that $\mu^{+}$, in case (a), (resp. $\mu^{-}$, in case (b)) is a smooth subvariety of $X$ and that

(d) $r_{+}: \mu_{+} \rightarrow \mu$, in case (a) (resp. $r_{-}: \mu_{-} \rightarrow \mu$, in case (b)) are naturally $H$ equivariant vector bundles. (If $X$ is projective, this follows from [BB]; the general 
case can be reduced to this case, as in [KL, 4.6], by using a smooth $H$-equivariant compactification of $X$, which exists by Hironaka.)

Moreover, one can arrange the connected components of $X^{\mathrm{C}^{*}}$ in a sequence $\mu_{1}, \ldots, \mu_{t}$ such that, for $j=1, \ldots, t$,

(e) $\mu_{\leq j}^{+}:=\mu_{1}^{+} \cup \mu_{2}^{+} \cup \cdots \cup \mu_{j}^{+}$is closed in $X$ (in case (a))

and

(f) $\mu_{\geq j}^{-}:=\mu_{t}^{-} \cup \mu_{t-1}^{-} \cup \cdots \cup \mu_{j}^{-}$is closed in $X$ (in case (b)).

Note that $\left(\mu_{j}^{+}\right)_{j \in[1, t]}$ is a partition of $X$ (in case (a)) and $\left(\mu_{j}^{-}\right)_{j \in[1, t]}$ is a partition of $X$ (in case (b)).

In case (a), let $j \in[1, t]$. Since $\mu_{j}^{+}$is an $H$-equivariant vector bundle over $\mu_{j}$ and $K_{H}\left(\mu_{j}\right)$ is a free $R_{H}$-module of finite rank, it follows that $K_{H}\left(\mu_{j}^{+}\right)$is a free $R_{H^{-}}$ module of the same rank as $K_{H}\left(\mu_{j}\right)$. Hence by $1.6, K_{H}(X)$ is a free $R_{H}$-module of the same rank as $\bigoplus_{j} K_{H}\left(\mu_{j}\right)=K_{H}\left(X^{\mathbf{C}^{*}}\right)$. This proves (a). The proof of (b) is entirely similar.

We prove (c). In this case, both $\mu^{+}, \mu^{-}$are defined for an irreducible component $\mu$ of $X^{\mathbf{C}^{*}}$ and both maps in (d) are defined. Moreover, we can arrange the connected components of $X^{\mathbf{C}^{*}}$ in a sequence $\mu_{1}, \ldots, \mu_{t}$ such that, for $j=1, \ldots, t$, both (e),(f) hold and also

(g) $\mu_{j}^{+} \cap \mu_{j^{\prime}}^{-}=\emptyset$ for $j<j^{\prime}$,

(h) $\mu_{j}^{+}, \mu_{j}^{-}$intersect transversally at $\mu_{j}$ for any $j$.

Let $j \in[1, t]$. We define a pairing $K_{H}\left(\mu_{\leq j}^{+}\right) \times K_{H}\left(\mu_{\geq j}^{-}\right) \rightarrow R_{H}$ as the composition

$$
K_{H}\left(\mu_{\leq j}^{+}\right) \times K_{H}\left(\mu_{\geq j}^{-}\right) \rightarrow K_{H}(X) \times K_{H}(X) \rightarrow R_{H}
$$

where the first map is given by the direct image under the inclusions and the second map is as in 1.5(a). Using ( $\mathrm{g}$ ), we see that this pairing is 0 on the image of

$$
K_{H}\left(\mu_{\leq j-1}^{+}\right) \times K_{H}\left(\mu_{\geq j}^{-}\right) \rightarrow K_{H}\left(\mu_{\leq j}^{+}\right) \times K_{H}\left(\mu_{\geq j}^{-}\right)
$$

and on the image of

$$
K_{H}\left(\mu_{\leq j}^{+}\right) \times K_{H}\left(\mu_{\geq j+1}^{-}\right) \rightarrow K_{H}\left(\mu_{\leq j}^{+}\right) \times K_{H}\left(\mu_{\geq j}^{-}\right)
$$

(given again by direct image). Hence it induces a pairing

$$
\left(K_{H}\left(\mu_{\leq j}^{+}\right) / K_{H}\left(\mu_{\leq j-1}^{+}\right)\right) \times\left(K_{H}\left(\mu_{\geq j}^{-}\right) / K_{H}\left(\mu_{\geq j+1}^{-}\right)\right) \rightarrow R_{H}
$$

or equivalently a pairing

$$
K_{H}\left(\mu_{j}^{+}\right) \times K_{H}\left(\mu_{j}^{-}\right) \rightarrow R_{H} .
$$

We identify $K_{H}\left(\mu_{j}^{+}\right)=K_{H}\left(\mu_{j}\right), K_{H}\left(\mu_{j}^{-}\right)=K_{H}\left(\mu_{j}\right)$ using the inverse image under the maps (d), with $\mu=\mu_{j}$. Then the last pairing becomes

(i) $K_{H}\left(\mu_{j}\right) \times K_{H}\left(\mu_{j}\right) \rightarrow R_{H}$.

Using (h) and the definitions, we see that this coincides with the pairing 1.5(a) for $X$ replaced by $\mu_{j}$. We see that the pairing 1.5(a) for $X$ may be identified with a pairing

$$
\bigoplus_{j} K_{H}\left(\mu_{j}\right) \times \bigoplus_{j} K_{H}\left(\mu_{j}\right) \rightarrow R_{H}
$$


of the form

$$
\left(\sum_{j} \xi_{j}, \sum_{j} \xi_{j}^{\prime}\right)=\sum_{j \geq j^{\prime}}\left(\xi_{j}, \xi_{j^{\prime}}^{\prime}\right)_{j, j^{\prime}}
$$

where $(,)_{j, j^{\prime}}: K_{H}\left(\mu_{j}\right) \times K_{H}\left(\mu_{j^{\prime}}\right) \rightarrow R_{H}$ are bilinear pairings defined for $j \geq j^{\prime}$ such that $(,)_{j, j}$ is as in (i). Since, by assumption, $(,)_{j, j}$ is perfect for all $j$, it follows that our pairing $K_{H}(X) \times K_{H}(X) \rightarrow R_{H}$ is perfect. The lemma is proved.

1.8. Throughout this paper we fix an $\mathfrak{s l}_{2}$-triple $(e, f, h)$ in $\mathfrak{g}$, that is, three elements $e, h, f$ of $\mathfrak{g}$ such that $[h, e]=2 e,[h, f]=-2 f,[e, f]=h$. Let $\zeta: S L_{2} \rightarrow G$ be the associated homomorphism as in [L5, 11.1].

Remark. In this paper, we shall refer a number of times to [L5] for definitions which make sense for our general $G$, even though in [L5], $G$ was assumed to be semisimple, simply-connected.

Let $\phi: \mathbf{C}^{*} \rightarrow G$ be the homomorphism defined by $\phi(\lambda)=\zeta\left(\begin{array}{cc}\lambda & 0 \\ 0 & \lambda^{-1}\end{array}\right)$. We have $\mathfrak{g}=\bigoplus_{n \in \mathbf{Z}} \mathfrak{g}_{n}$ where $\mathfrak{g}_{n}=\{x \in \mathfrak{g} \mid[h, x]=n x\}$. Let

$$
\mathcal{B}_{e}=\{\mathfrak{b} \in \mathcal{B} \mid e \in \mathfrak{b}\} .
$$

For any subspace $\mathfrak{m}$ of $\mathfrak{g}$ we set $\mathfrak{z}_{\mathfrak{m}}(f)=\{x \in \mathfrak{m} \mid[x, f]=0\}$. Let

$$
\begin{gathered}
\Sigma_{e}^{\prime}=\left\{y \in \mathfrak{g} \mid y-e \in \mathfrak{z}_{\mathfrak{g}}(f)\right\}, \quad \Sigma_{e}=\Sigma_{e}^{\prime} \cap \mathfrak{g}_{n i l}, \\
\Lambda_{e}^{\prime}=\left\{(y, \mathfrak{b}) \in \Sigma_{e}^{\prime} \times \mathcal{B} \mid y \in \mathfrak{b}\right\}, \quad \Lambda_{e}=\left\{(y, \mathfrak{b}) \in \Sigma_{e} \times \mathcal{B} \mid y \in \mathfrak{b}\right\} .
\end{gathered}
$$

It is known that $\Lambda_{e}$ is smooth [S, p. 65], irreducible. We have inclusions $\mathcal{B}_{e} \subset \Lambda_{e} \subset$ $\Lambda_{e}^{\prime}$; the first inclusion is $\mathfrak{b} \mapsto(e, \mathfrak{b})$; the second inclusion is the obvious one.

Let $C$ be a maximal torus of the simultaneous centralizer of $e, f, h$ in $G$. Let $H=C \times \mathbf{C}^{*}$. From now on, the notation $\mathfrak{R}, M_{\mathfrak{R}}$ in 1.1 will refer to this $H$.

The group $H$ acts on $\mathcal{B}_{e}, \mathfrak{g}, \Lambda_{e}, \Lambda_{e}^{\prime}$ by restriction of the action of $G \times \mathbf{C}^{*}$ on $\mathcal{B}, \mathfrak{g}, \Lambda, \Lambda^{\prime}$, where we regard $H$ as a subgroup of $G \times \mathbf{C}^{*}$ by $(c, \lambda) \mapsto(c \phi(\lambda), \lambda)$. In particular, the $H$-action on $\mathfrak{g}$ is

$$
(c, \lambda): y \mapsto \lambda^{-2} \operatorname{Ad}(c \phi(\lambda)) y
$$

and the $H$-action on $\mathcal{B}$ is

$$
(c, \lambda): \mathfrak{b} \mapsto \lambda^{-2} \operatorname{Ad}(c \phi(\lambda)) \mathfrak{b}=\operatorname{Ad}(c \phi(\lambda)) \mathfrak{b} .
$$

The subvarieties $\{e\}, \mathfrak{z}_{\mathfrak{g}}(f), \Sigma_{e}^{\prime}, \Sigma_{e}$ of $\mathfrak{g}$ are $H$-stable.

Any variety with $H$-action will be also regarded as a variety with $\mathbf{C}^{*}$-action, by restricting the $H$-action to $\mathbf{C}^{*}$ via $\mathbf{C}^{*} \rightarrow H, \lambda \mapsto(1, \lambda)$.

Taking the fixed point sets of $H$ on $\mathcal{B}_{e}, \Lambda_{e}, \Lambda_{e}^{\prime}$ we obtain

(a) $\mathcal{B}_{e}^{H}=\Lambda_{e}^{H}=\Lambda_{e}^{\prime H}$.

In fact we have the stronger statement that already the fixed point sets of $\mathbf{C}^{*}$ coincide:

(b) $\mathcal{B}_{e}^{\mathbf{C}^{*}}=\Lambda_{e}^{\mathbf{C}^{*}}=\Lambda_{e}^{\prime \mathbf{C}^{*}}$.

This is because the $\mathbf{C}^{*}$-action on $\mathfrak{z}_{\mathfrak{g}}(f)$ has all weights $<0$, hence $\mathfrak{z}_{\mathfrak{g}}(f) \mathbf{C}^{*}=\{0\}$.

The varieties in (a),(b) are both smooth and projective, since $\Lambda_{e}$ is smooth and $\mathcal{B}_{e}$ is projective.

Lemma 1.9 ([DLP, 3.9]). Any connected component of the fixed point set $\mathcal{B}_{e}^{H}$ has property $(S)$. 
Lemma 1.10. Let $\mu$ be a connected component of the fixed point set $\mathcal{B}_{e}^{\mathbf{C}^{*}}=\Lambda_{e}^{\mathbf{C}^{*}}$ with its induced $H$-action. Then the $R_{H}$-modules $K_{H}(\mu), K_{H}\left(\mu^{H}\right)$ are free of the same (finite) rank.

We apply Lemma 1.7(a) with $X=\mu$ and for a homomorphism $\tau: \mathbf{C}^{*} \rightarrow H$ which is in general position so that the fixed point set $\mu^{\tau\left(\mathbf{C}^{*}\right)}$ is equal to $\mu^{H}$. That lemma is applicable since, for each connected component $\mu^{\prime}$ of $\mu^{H}$ we have $K_{H}\left(\mu^{\prime}\right)=R_{H} \otimes K\left(\mu^{\prime}\right)$ (since $H$ acts trivially on $\mu^{\prime}$ ) and $R_{H} \otimes K\left(\mu^{\prime}\right)$ is a free $R_{H^{-}}$ module of finite rank by Lemmas $1.9,1.3$. We deduce that the $R_{H}$-module $K_{H}(\mu)$ is a free $R_{H}$-module of the same (finite) rank as $K_{H}\left(\mu^{H}\right)$. The lemma follows.

Lemma 1.11. (a) The $\mathbf{C}^{*}$-action on $\Lambda_{e}$ is, for $\lambda \rightarrow \infty$, a contraction to the compact subvariety $\mathcal{B}_{e}^{\mathbf{C}^{*}}$ of $\Lambda_{e}$.

(b) The $R_{H}$-modules $K_{H}\left(\Lambda_{e}\right), K_{H}\left(\Lambda_{e}^{H}\right)$ are free of the same finite rank.

(a) follows immediately from the fact that the weights of the $\mathbf{C}^{*}$-action on $\mathfrak{z}_{\mathfrak{g}}(f)$ are $<0$. To prove (b), we apply Lemma $1.7(\mathrm{~b})$ with $X=\Lambda_{e}$. That lemma is applicable in view of (a) and 1.10. The lemma is proved.

1.12. Let $\mathfrak{q}=\bigoplus_{n>0} \mathfrak{g}_{n}$. Then $\mathfrak{q}$ is a parabolic subalgebra of $\mathfrak{g}$ and $\mathfrak{n}_{\mathfrak{q}}=\bigoplus_{n>0} \mathfrak{g}_{n}$. Let $Q, U_{Q}$ be the closed connected subgroups of $G$ corresponding to $\mathfrak{q}, \mathfrak{n}_{\mathfrak{q}}$. Then $C$ and $\phi\left(\mathbf{C}^{*}\right)$ are subgroups of $Q$. Hence, if $\mathbf{O}$ is a $Q$-orbit on $\mathcal{B}$, then $\mathbf{O}$ is an $H$-stable subvariety of $\mathcal{B}$. In particular, $\mathbf{O}$ has a $\mathbf{C}^{*}$-action (see 1.8 ).

Lemma 1.13. (a) This $\mathbf{C}^{*}$-action is, for $\lambda \rightarrow 0$, a contraction of $\mathbf{O}$ to its compact subvariety $\mathbf{O}^{\mathbf{C}^{*}}$.

(b) The restriction of the $\mathbf{C}^{*}$-action above from $\mathbf{O}$ to its closed subvariety $\mathcal{B}_{e} \cap \mathbf{O}$ is, for $\lambda \rightarrow 0$, a contraction of $\mathcal{B}_{e} \cap \mathbf{O}$ to $\mathcal{B}_{e} \cap \mathbf{O}^{\mathbf{C}^{*}}=\left(\mathcal{B}_{e} \cap \mathbf{O}\right)^{\mathbf{C}^{*}}$.

(c) The $R_{H}$-modules $K_{H}\left(\mathcal{B}_{e} \cap \mathbf{O}\right), K_{H}\left(\mathcal{B}_{e}^{H} \cap \mathbf{O}\right)$ are free of the same finite rank.

(d) The $R_{H}$-modules $K_{H}\left(\mathcal{B}_{e}\right), K_{H}\left(\mathcal{B}_{e}^{H}\right)$ are free of the same finite rank.

We prove (a). We denote our $\mathbf{C}^{*}$-action on $\mathcal{B}$ by $\lambda, \mathfrak{b} \mapsto \lambda \circ \mathfrak{b}$. If $\mathfrak{b} \in \mathbf{O}$, then $\mathfrak{b}=\operatorname{Ad}(u) \mathfrak{b}_{1}$ for some $u \in U_{Q}$ and $\mathfrak{b}_{1} \in \mathbf{O}^{\mathbf{C}^{*}}$. We have $\lambda \circ \mathfrak{b}=\operatorname{Ad}\left(u_{\lambda}\right)\left(\mathfrak{b}_{1}\right)$ where $u_{\lambda}=\phi(\lambda)(u) \phi(\lambda)^{-1} \in U_{Q}$. From the definition of $U_{Q}$ we see that $\lim _{\lambda \rightarrow 0} u_{\lambda}=1$ in $U_{Q}$. Hence (a) holds. Now (b) follows immediately from (a).

We prove (c). Note that any connected component of $\left(\mathcal{B}_{e} \cap \mathbf{O}\right)^{\mathbf{C}^{*}}$ is also a connected component of $\mathcal{B}_{e}^{\mathbf{C}^{*}}$. (Indeed, when $\mathbf{O}$ varies, the sets $\left(\mathcal{B}_{e} \cap \mathbf{O}\right)^{\mathbf{C}^{*}}$ form a partition of $\mathcal{B}_{e}^{\mathbf{C}^{*}}$ into closed subsets.) Hence, by 1.10 , for any connected component $\mu$ of $\left(\mathcal{B}_{e} \cap \mathbf{O}\right)^{\mathbf{C}^{*}}$, the $R_{H}$-module $K_{H}(\mu)$ is free of finite rank. We can therefore apply 1.7(a) with $X=\mathcal{B}_{e} \cap \mathbf{O}$, which is smooth by [DLP, 3.4]. (Here we use (b).) We deduce that $K_{H}\left(\mathcal{B}_{e} \cap \mathbf{O}\right)$ is a free $R_{H}$-module of the same rank as $K_{H}\left(\left(\mathcal{B}_{e} \cap \mathbf{O}\right)^{\mathbf{C}^{*}}\right)$. Using again 1.10, we have that $K_{H}\left(\mu^{H}\right)$ is free over $R_{H}$ of the same rank as $K_{H}(\mu)$, for any $\mu$ as above. Taking disjoint union over all $\mu$, we deduce that

$$
\left.K_{H}\left(\left(\left(\mathcal{B}_{e} \cap \mathbf{O}\right)^{\mathbf{C}^{*}}\right)^{H}\right)=K_{H}\left(\left(\mathcal{B}_{e} \cap \mathbf{O}\right)\right)^{H}\right)
$$

is free over $R_{H}$ of the same $\operatorname{rank} K_{H}\left(\left(\mathcal{B}_{e} \cap \mathbf{O}\right)^{\mathbf{C}^{*}}\right)$. This proves (c).

Now (d) follows from (c) for various $\mathbf{O}$, using 1.6. The lemma is proved.

The following result has been conjectured and proved in some special cases in $[\mathrm{L} 5, \S 11]$.

Theorem 1.14. (a) The $R_{H}$-modules $K_{H}\left(\mathcal{B}_{e}\right)$ and $K_{H}\left(\Lambda_{e}\right)$ are free of rank equal to $\chi_{\mathcal{B}_{e}^{H}}=\chi_{\mathcal{B}_{e}}$. 
(b) The canonical maps $K_{H}\left(\mathcal{B}_{e}^{H}\right) \rightarrow K_{H}\left(\mathcal{B}_{e}\right), K_{H}\left(\Lambda_{e}^{H}\right) \rightarrow K_{H}\left(\Lambda_{e}\right)$ (direct image) induced by the inclusions $\mathcal{B}_{e}^{H} \subset \mathcal{B}_{e}, \Lambda_{e}^{H} \subset \Lambda_{e}$ are injective. They induce isomorphisms

$$
K_{H}\left(\mathcal{B}_{e}^{H}\right)_{\mathfrak{R}} \stackrel{\sim}{\rightarrow} K_{H}\left(\mathcal{B}_{e}\right)_{\mathfrak{R}}, \quad K_{H}\left(\Lambda_{e}^{H}\right)_{\mathfrak{R}} \stackrel{\sim}{\rightarrow} K_{H}\left(\Lambda_{e}\right)_{\mathfrak{R}}
$$

(c) The canonical map $K_{H}\left(\mathcal{B}_{e}\right) \rightarrow K_{H}\left(\Lambda_{e}\right)$ (direct image) induced by the inclusion $\mathcal{B}_{e} \subset \Lambda_{e}$ is injective. It induces an isomorphism $K_{H}\left(\mathcal{B}_{e}\right)_{\mathfrak{R}} \stackrel{\sim}{\rightarrow} K_{H}\left(\Lambda_{e}\right)_{\mathfrak{R}}$.


are free of the same rank. The method of proof of $1.13(\mathrm{~d})$ also yields that $\chi_{\mathcal{B}_{e}^{H}}=$ $\chi_{\mathcal{B}_{e}}$. It remains to show that $\operatorname{rk} K_{H}\left(\mathcal{B}_{e}^{H}\right)=\chi_{\mathcal{B}_{e}^{H}}$. This follows from 1.9 and 1.3. This proves (a).

The second sentence in (b) follows from [Th]. The first sentence in (b) follows from the second sentence since, by (a), the natural maps

$$
K_{H}\left(\mathcal{B}_{e}^{H}\right) \rightarrow K_{H}\left(\mathcal{B}_{e}^{H}\right)_{\Re}, K_{H}\left(\Lambda_{e}^{H}\right) \rightarrow K_{H}\left(\Lambda_{e}^{H}\right)_{\mathfrak{R}}
$$

are injective. The second sentence in (c) follows from (b) since $\mathcal{B}_{e}^{H}=\Lambda_{e}^{H}$. The first sentence in (c) follows from the second sentence since we have naturally $K_{H}\left(\mathcal{B}_{e}\right) \subset$ $K_{H}\left(\mathcal{B}_{e}\right)_{\mathfrak{R}}, K_{H}\left(\Lambda_{e}\right) \subset K_{H}\left(\Lambda_{e}\right)_{\mathfrak{R}}$ by the freeness statement in (a). The theorem is proved.

1.15. We shall identify the $\mathfrak{R}$-vector spaces $K_{H}\left(\mathcal{B}_{e}\right)_{\mathfrak{R}}$ and $K_{H}\left(\Lambda_{e}\right)_{\mathfrak{R}}$ via $1.14(\mathrm{c})$; we denote them by $\mathfrak{E}_{e}$. Then we have naturally $K_{H}\left(\mathcal{B}_{e}\right) \subset K_{H}\left(\Lambda_{e}\right) \subset \mathfrak{E}_{e}$.

1.16. Now let $\tilde{G} \rightarrow G$ be a surjective homomorphism of algebraic groups with central kernel and with $\tilde{G}$ connected, reductive. Then $e, h, f$ gives rise canonically to an analogous triple denoted again by $e, h, f$ in the Lie algebra of $\tilde{G}$. We can naturally identify the varieties $\mathcal{B}_{e}, \Lambda_{e}$ defined in terms of $G$ with those defined in terms of $\tilde{G}$. Let $\tilde{C}$ be the maximal torus of the simultaneous centralizer of $e, h, f$ in $\tilde{G}$ whose image in $G$ equals $C$. Let $\tilde{H}=\tilde{C} \times \mathbf{C}^{*}$. We have an obvious (injective) ring homomorphism $R_{H} \rightarrow R_{\tilde{H}}$ and obvious $R_{H}$-linear maps

$$
K_{H}\left(\mathcal{B}_{e}\right) \rightarrow K_{\tilde{H}}\left(\mathcal{B}_{e}\right), K_{H}\left(\Lambda_{e}\right) \stackrel{\sim}{\rightarrow} K_{\tilde{H}}\left(\Lambda_{e}\right) .
$$

Proposition 1.17. These maps induce isomorphisms of $R_{H}$-modules

(a) $R_{\tilde{H}} \otimes_{R_{H}} K_{H}\left(\mathcal{B}_{e}\right) \stackrel{\sim}{\rightarrow} K_{\tilde{H}}\left(\mathcal{B}_{e}\right)$,

(b) $R_{\tilde{H}} \otimes_{R_{H}} K_{H}\left(\Lambda_{e}\right) \stackrel{\sim}{\rightarrow} K_{\tilde{H}}\left(\Lambda_{e}\right)$.

By partitioning $\mathcal{B}_{e}, \Lambda_{e}$ as in the proofs of $1.11,1.13$ (which rely on $1.7,1.10$ ) and using short exact sequences as in 1.6, we are reduced to proving the analogous result in the case where $\mathcal{B}_{e}, \Lambda_{e}$ are replaced by a connected component $\mu$ of $\mathcal{B}_{e}^{H}=$ $\Lambda_{e}^{H}=\mathcal{B}_{e}^{\tilde{H}}=\Lambda_{e}^{\tilde{H}}$. In this case the result is obvious since $H, \tilde{H}$ act trivially on $\mu$ so that $K_{H}(\mu)=R_{H} \otimes K(\mu), K_{\tilde{H}}(\mu)=R_{\tilde{H}} \otimes K(\mu)$.

1.18. In the next lemma we will restrict the $H$ actions to $\mathbf{C}^{*}$ as in 1.8 .

Lemma 1.19. The natural homomorphisms $R_{\mathbf{C}^{*}} \otimes_{R_{H}} K_{H}\left(\mathcal{B}_{e}\right) \stackrel{\sim}{\rightarrow} K_{\mathbf{C}^{*}}\left(\mathcal{B}_{e}\right)$, $R_{\mathbf{C}^{*}} \otimes_{R_{H}} K_{H}\left(\Lambda_{e}\right) \stackrel{\sim}{\rightarrow} K_{\mathbf{C}^{*}}\left(\Lambda_{e}\right)$ are isomorphisms of $R_{\mathbf{C}^{*}-\text { modules. }}$

The proof is similar to that of 1.17 . 


\section{PERfECTNESS OF A BILINEAR PAIRING}

2.1. Let $\mathfrak{b} \in \mathcal{B}$. Let $\mathbf{O}$ be the $Q$-orbit of $\mathfrak{b}$. Then $\mathcal{B}_{e} \cap \mathbf{O}$ is smooth (see [DLP, 3.4]). We may regard $\mathcal{B}_{e} \cap \mathbf{O}$ as a submanifold of $\Lambda_{e}$. Thus the tangent space $\mathcal{T}^{\prime}$ of $\mathcal{B}_{e} \cap \mathbf{O}$ at $\mathfrak{b}$ is naturally a subspace of the tangent space $\mathcal{T}$ of $\Lambda_{e}$ at $(e, \mathfrak{b})$ which is itself naturally a subspace of the tangent space $\mathcal{T}_{0}$ of $\Lambda$ at $(e, \mathfrak{b})$.

Assume now that, in addition, we have $h \in \mathfrak{b}$. Then $(e, \mathfrak{b}) \in \Lambda^{\mathbf{C}^{*}}$. Then $\mathbf{C}^{*}$ acts naturally on $\mathcal{T}$ and this action preserves the subspace $\mathcal{T}^{\prime}$. Hence there is an induced $\mathbf{C}^{*}$-action on $\mathcal{T} / \mathcal{T}^{\prime}$.

Proposition 2.2. All weights of the $\mathbf{C}^{*}$-action on $\mathcal{T} / \mathcal{T}^{\prime}$ are $<0$.

$\theta(\xi, \eta)=([\xi, e]-\eta, \xi \bmod \mathfrak{b})$ is a linear map $\theta: \mathfrak{g} \times \mathfrak{n}_{\mathfrak{b}} \rightarrow \mathfrak{g} \times(\mathfrak{g} / \mathfrak{b})$. We have canonically

$$
\mathcal{T}_{0}=\operatorname{Im}(\theta)=\mathfrak{g} \times \mathfrak{n}_{\mathfrak{b}} / \operatorname{Ker}(\theta)=\frac{\mathfrak{g} \times \mathfrak{n}_{\mathfrak{b}}}{\{(\zeta,[\zeta, e]) \mid \zeta \in \mathfrak{b}\}} .
$$

Now $\mathcal{T}=\left\{\left(\xi^{\prime}, \eta^{\prime}\right) \in \operatorname{Im}(\theta) \mid\left[\xi^{\prime}, f\right]=0\right\} \subset \mathcal{T}_{0}$. Hence

$$
\mathcal{T}=\tilde{\mathcal{T}} /\{(\zeta,[\zeta, e]) \mid \zeta \in \mathfrak{b}\}
$$

where $\tilde{\mathcal{T}}=\left\{(\xi, \eta) \in \mathfrak{g} \times \mathfrak{n}_{\mathfrak{b}} \mid[[\xi, e]-\eta, f]=0\right\}$. From the definition we have

$$
\mathcal{T}^{\prime}=\tilde{\mathcal{T}}^{\prime} / \mathfrak{b} \text { where } \tilde{\mathcal{T}}^{\prime}=\{\xi \in \mathfrak{q} \mid[\xi, e] \in \mathfrak{b}\} .
$$

The inclusion $\mathcal{T}^{\prime} \subset \mathcal{T}$ with $\mathcal{T}$ is induced by the inclusion $\tilde{\mathcal{T}}^{\prime} \rightarrow \tilde{\mathcal{T}}$ given by $\xi \mapsto$ $(\xi,[\xi, e])$. It follows that $\mathcal{T} / \mathcal{T}^{\prime}=\tilde{\mathcal{T}} / \tilde{\mathcal{T}}^{\prime}$. We have exact sequences

$$
0 \rightarrow \mathfrak{z}_{\mathfrak{g}}(e) \rightarrow \tilde{\mathcal{T}} \rightarrow \mathfrak{n}_{\mathfrak{b}} \rightarrow 0
$$

(the first map is $\zeta \mapsto(\zeta, 0)$; the second map is $(\xi, \eta) \mapsto \eta)$ and

$$
0 \rightarrow \mathfrak{z}_{\mathfrak{g}}(e) \rightarrow \tilde{\mathcal{T}}^{\prime} \rightarrow \mathfrak{n}_{\mathfrak{b}} \cap \bigoplus_{n \geq 2} \mathfrak{g}_{n} \rightarrow 0
$$

(the first map is $\zeta \mapsto \zeta$; the second map is $\xi \mapsto[\xi, e]$ ). The exactness of the first sequence follows from the equality $\mathfrak{g}=\operatorname{Im} \operatorname{ad}(e) \oplus \operatorname{Ker} \operatorname{ad}(f)$. The exactness of the second sequence follows from the equality $[\mathfrak{q}, e]=\bigoplus_{n \geq 2} \mathfrak{g}_{n}$. (Both follow from the representation theory of $\mathfrak{s l}_{2}$.)

From the exact sequences above it follows that

$$
\tilde{\mathcal{T}} / \tilde{\mathcal{T}}^{\prime}=\mathfrak{n}_{\mathfrak{b}} /\left(\mathfrak{n}_{\mathfrak{b}} \cap \bigoplus_{n \geq 2} \mathfrak{g}_{n}\right)=\mathfrak{n}_{\mathfrak{b}} \cap \bigoplus_{n \leq 1} \mathfrak{g}_{n} .
$$

Thus the $\mathbf{C}^{*}$-action on $\mathcal{T} / \mathcal{T}^{\prime}$ corresponds to the $\mathbf{C}^{*}$-action on $\mathfrak{n}_{\mathfrak{b}} \cap \bigoplus_{n \leq 1} \mathfrak{g}_{n}$ (restriction of the $\mathbf{C}^{*}$-action on $\mathfrak{g}$ ). This action restricted to $\mathfrak{g}_{n}$ is by multiplication by $\lambda^{-2+n}$. Since $-2+n<0$ for $n \leq 1$, the proposition is proved.

Corollary 2.3. Let us write $\mathcal{T}=\mathcal{T}^{<0} \oplus \mathcal{T}^{0} \oplus \mathcal{T}>0, \mathcal{T}^{\prime}=\mathcal{T}^{\prime<0} \oplus \mathcal{T}^{\prime 0} \oplus \mathcal{T}^{\prime>0}$, where $\mathcal{T}^{<0}, \mathcal{T}^{\prime<0}$ (resp. $\mathcal{T}^{>0}, \mathcal{T}^{\prime>0}$ ) denote the the sum of $<0$ (resp. $>0$ ) weight spaces of the $\mathbf{C}^{*}$-action and $\mathcal{T}^{0}, \mathcal{T}^{\prime 0}$ denote the 0 -weight spaces. Then

(a) $\mathcal{T}^{0}=\mathcal{T}^{\prime 0}$,

(b) $\mathcal{T}^{>0}=\mathcal{T}^{\prime>0}$. 
2.4. Let (:) $: K_{H}\left(\mathcal{B}_{e}\right) \times K_{H}\left(\Lambda_{e}\right) \rightarrow R_{H}$ be the $R_{H}$-bilinear pairing defined in [L5, 12.11].

Theorem 2.5. (a) (:) with scalars extended from $R_{H}$ to $\mathbf{Q} \otimes R_{H}$ is perfect.

(b) Assume that any irreducible component of $\mathcal{B}_{e}^{H}$ has property $\left(S^{\prime}\right)$. (See 1.4.) Then (:) itself is perfect.

We prove (b). Let $X$ be a smooth $H$-equivariant compactification of $\Lambda_{e}$. We consider the $\mathbf{C}^{*}$-action on $X$ given by the restriction of the $H$-action, as in 1.8. For any connected component $\mu$ of $X^{\mathrm{C}^{*}}$ we consider the smooth submanifolds $\mu_{+}, \mu^{-}$ of $X$ and the vector bundle maps $r_{+}: \mu_{+} \rightarrow \mu, r_{-}: \mu_{-} \rightarrow \mu$ as in 1.7(d). We can arrange the connected components of $X^{\mathbf{C}^{*}}$ in a sequence $\mu_{1}, \ldots, \mu_{t}$ such that, for $j=1, \ldots, t, 1.7(\mathrm{e}),(\mathrm{f}),(\mathrm{g}),(\mathrm{h})$ hold. Note that $\left(\mu_{j}^{+}\right)_{j \in[1, t]}$ and $\left(\mu_{j}^{-}\right)_{j \in[1, t]}$ are two partitions of $X$. Since the $\mathbf{C}^{*}$-action is, for $\lambda \rightarrow \infty$ a contraction of $\Lambda_{e}$ to a compact subset of $\Lambda_{e}$, we see that, for $j \in[1, t]$, we have either $\mu_{j} \subset \Lambda_{e}$ or $\mu_{j} \subset X-\Lambda_{e}$. Let $J$ be the set of $j \in[1, t]$ for which the first alternative holds. For $j \in J$ we have

$$
\mu_{j}^{-}=\left\{x \in \Lambda_{e} \mid \lim _{\lambda \rightarrow \infty} \lambda x \in \mu_{j}\right\}
$$

(using again the contraction property on $\Lambda_{e}$ ). Since, for any $Q$-orbit $\mathbf{O}$ on $\mathcal{B}$, the set $\mathcal{B}_{e}^{\mathbf{C}^{*}} \cap \mathbf{O}$ is compact, we see that for any $j \in J$ we have $\mu_{j} \subset \mathcal{B}_{e}^{\mathbf{C}^{*}} \cap \mathbf{O}$ for a unique $\mathbf{O}$ as above. Let $\mu_{j}^{+}=\left\{x \in \mathcal{B}_{e} \cap \mathbf{O} \mid \lim _{\lambda \rightarrow 0} \lambda x \in \mu_{j}\right\}$.

By 1.13(b) and the proof of 1.7(a), the sets $\mu_{j}^{\prime+}$ (with $j \in J$ ) form a partition of $\mathcal{B}_{e}$ and each $\mu_{j}^{\prime+}$ is naturally a vector bundle over $\mu_{j}$ with fibres of dimension equal to $\operatorname{dim} \mathcal{T}^{\prime>0}$. On the other hand, $\mu_{j}^{+}$is naturally a vector bundle over $\mu_{j}$ with fibres of dimension equal to $\operatorname{dim} \mathcal{T}^{>0}$. Since $\mu_{j}^{\prime+} \subset \mu_{j}^{+}$and $\operatorname{dim} \mathcal{T}^{\prime>0}=\operatorname{dim} \mathcal{T}>0$, (see 2.3), it follows that we must have $\mu_{j}^{+}=\mu_{j}^{+}$for any $j \in J$.

Thus, the $\mu_{j}^{+}$with $j \in J$ form a partition of $\mathcal{B}_{e}$. On the other hand, the $\mu_{j}^{-}$ with $j \in J$ form a partition of $\mathcal{B}_{e}$. Let us write $J=\left\{j_{1}<j_{2}<\cdots<j_{p}\right\}$. Then properties 1.7(e)-(h) of the $\mu_{j}^{ \pm}$imply the following: $\mu_{j_{1}}^{+} \cup \mu_{j_{2}}^{+} \cup \cdots \cup \mu_{j_{k}}^{+}$is closed in $\mathcal{B}_{e}$ for $k \in[1, p]$ (since it is the intersection of $\mu_{1}^{+} \cup \mu_{2}^{+} \cup \cdots \cup \mu_{j_{k}}^{+}$with $\mathcal{B}_{e}$ ); $\mu_{j_{p}}^{-} \cup \mu_{j_{p-1}}^{-} \cup \cdots \cup \mu_{j_{k}}^{-}$is closed in $\Lambda_{e}$ for $k \in[1, p]$ (equivalently: $\mu_{j_{1}}^{-} \cup \mu_{j_{2}}^{-} \cup \cdots \cup$ $\mu_{j_{k-1}}^{-}$is open in $\Lambda_{e}$, since it is the intersection of $\mu_{1}^{-} \cup \mu_{2}^{-} \cup \cdots \cup \mu_{j_{k-1}}^{-}$with $\Lambda_{e}$ ); $\mu_{j_{k}}^{+} \cap \mu_{j_{k^{\prime}}}^{-}=\emptyset$ for $k<k^{\prime}$, (since $j_{k}<j_{k^{\prime}}$ ); $\mu_{j_{k}}^{+}, \mu_{j_{k}}^{-}$intersect transversally at $\mu_{j_{k}}$ for any $k$. Repeating now the argument in the proof of $1.7(\mathrm{c})$, we see that the pairing $(:): K_{H}\left(\mathcal{B}_{e}\right) \times K_{H}\left(\Lambda_{e}\right) \rightarrow R_{H}$ may be identified with a pairing

$$
\bigoplus_{k} K_{H}\left(\mu_{j_{k}}\right) \times \oplus_{k} K_{H}\left(\mu_{j_{k}}\right) \rightarrow R_{H}
$$

of the form

$$
\left(\sum_{k} \xi_{k}, \sum_{k} \xi_{k}^{\prime}\right)=\sum_{k>k^{\prime}}\left(\xi_{j_{k}}, \xi_{j_{k^{\prime}}}^{\prime}\right)_{k, k^{\prime}}
$$

where $(,)_{k, k^{\prime}}: K_{H}\left(\mu_{j_{k}}\right) \times K_{H}\left(\mu_{j_{k^{\prime}}}\right) \rightarrow R_{H}$ are bilinear pairings defined for $k \geq k^{\prime}$ such that $(,)_{k, k}$ is as in $1.5(\mathrm{a})$.

Hence to prove that (:) is perfect, it is enough to show that each $(,)_{k, k}$ is perfect. We are reduced to showing that any connected component $\mu$ of $\mathcal{B}_{e}^{\mathrm{C}^{*}}$ satisfies $\left(S_{H}^{\prime}\right)$ (see 1.5). 
Let $\tau: \mathbf{C}^{*} \rightarrow H$ be as in the proof of Lemma 1.10. Using Lemma 1.7(c) we are reduced to showing that each connected component of $\mu^{\tau\left(\mathbf{C}^{*}\right)}=\mu^{H}$ has property $\left(S_{H}^{\prime}\right)$. Since $H$ acts trivially on that component, it is enough to show that the component has property $\left(S^{\prime}\right)$. But this is just the assumption of (b). Hence (b) is proved.

Now the proof of (a) is entirely similar; it uses the fact that property $\left(S^{\prime}\right)$ is automatically satisfied over $\mathbf{Q}$ in the presence of property $(S)$ (see 1.4). The theorem is proved.

2.6. The $R_{H}$-bilinear pairing (:) in 2.4 extends uniquely to an $\mathfrak{R}$-bilinear pairing $\mathfrak{E}_{e} \times \mathfrak{E}_{e} \rightarrow \mathfrak{R}$ (see 1.15) which will be denoted again by (:).

Lemma 2.7. This pairing is perfect and symmetric.

The fact that it is perfect follows from 2.5. To show that it is symmetric, it suffices to show that its restriction $K_{H}\left(\mathcal{B}_{e}\right) \times K_{H}\left(\mathcal{B}_{e}\right) \rightarrow R_{H}$ is symmetric. This restriction attaches to $F, F^{\prime} \in \operatorname{Coh}_{H}\left(\mathcal{B}_{e}\right)$ the element $\pi_{*}\left(F \otimes_{\Lambda_{e}}^{L} F^{\prime}\right)$ where $\otimes_{\Lambda_{e}}^{L}$ is a Tor-product as in [L5, 6.4] relative to the smooth manifold $\Lambda_{e}$ and its closed subvarieties $\mathcal{B}_{e}, \mathcal{B}_{e}$ and $\pi$ is the map from $\mathcal{B}_{e}$ to the point. This is obviously symmetric in $F, F^{\prime}$. The lemma is proved.

\section{The Element $\nabla_{e} \in R_{H}$}

3.1. The one-dimensional representation $p r_{2}: G \times \mathbf{C}^{*} \rightarrow \mathbf{C}^{*}$ will be denoted by $v$ (regarded as an indeterminate). Similarly, the restriction of this representation to a closed subgroup of $G \times \mathbf{C}^{*}$ (such as $H$ ) is denoted by $v$. Thus, we have $v \in R_{H}$ and the direct product decomposition $H=C \times \mathbf{C}^{*}$ defines an isomorphism of rings $R_{H}=R_{C}\left[v, v^{-1}\right]$. This makes $R_{H}$ into an $\mathcal{A}$-algebra. In particular, any $R_{H^{-}}$ module, for example $K_{H}(X)$ where $X$ is a variety with $H$-action, can be naturally regarded as an $\mathcal{A}$-module.

For any (finite dimensional) $H$-module $V$ we set

$$
V=\sum(-1)^{t} V^{(t)} \in R_{H}
$$

where $V^{(t)}$ is the $t$-th exterior power of $V$. Let $V^{*}$ be the dual $H$-module. We set $\Omega_{V}=V^{*(d)}$ where $d=\operatorname{dim} V$. We have

(a) $V^{*}=(-1)^{d} \Omega_{V} V$ where $d=\operatorname{dim} V$.

Let $V^{\dagger}$ be the $H$-module with underlying space $V$ in which $(c, \lambda)$ acts as $\left(c^{-1}, \lambda\right)$ in the original $H$-action on $V$. This extends by linearity to a ring involution ${ }^{\dagger}$ : $R_{H} \rightarrow R_{H}$. Let ${ }^{-}: R_{H} \rightarrow R_{H}$ be the ring involution which maps an $H$-module $V$ to $\left(V^{*}\right)^{\dagger}=\left(V^{\dagger}\right)^{*}$.

Let $\mathfrak{t}$ be the abstract Cartan algebra of $\mathfrak{g}$. For any $\mathfrak{b} \in \mathcal{B}$ we have naturally $\mathfrak{b} / \mathfrak{n}_{\mathfrak{b}}=\mathfrak{t}$. The group $H$ acts on $\mathfrak{t}$ by $(c, \lambda): t \mapsto \lambda^{-2} t$. Hence $\mathfrak{t}, \mathfrak{t}^{*}$ are well defined elements of $R_{H}$. Note that $\mathfrak{t}=\left(1-v^{-2}\right)^{r}, \mathfrak{t}^{*}=\left(1-v^{2}\right)^{r}$ where $r=\operatorname{dim} \mathfrak{t}$.

Note that $\mathfrak{z}_{\mathfrak{g}}(f)$ is an $H$-submodule of $\mathfrak{g}$. Hence $\mathfrak{z}_{\mathfrak{g}}(f), \mathfrak{z}_{\mathfrak{g}}(f)^{*}$ are well defined elements of $R_{H}$. Let

$$
\nabla_{e}=\mathfrak{z}_{\mathfrak{g}}(f) \cdot \mathbb{t}^{-1} \in \mathfrak{R}, \quad \nabla_{e}^{\prime}=\mathfrak{z}_{\mathfrak{g}}(f)^{*} \cdot \mathfrak{t}^{*} \in \mathfrak{R} .
$$

Lemma 3.2. (a) We have $\nabla_{e} \in 1+v^{-1} R_{C}\left[v^{-1}\right] \subset R_{H}$.

(b) We have $\nabla_{e}^{\dagger}=\nabla_{e}$. 
We prove (a). Since $\mathfrak{z}_{\mathfrak{g}}(f) \subset \bigoplus_{n \leq 0} \mathfrak{g}_{n}$, we see that the $H$-module $\mathfrak{z}_{\mathfrak{g}}(f)$ is of the form $\bigoplus_{\rho, m<0} V_{\rho, m} \otimes \rho \otimes v^{m}$ where $\rho$ runs over the characters $C \rightarrow \mathbf{C}^{*}$ and $V_{\rho, m}$ are $\mathbf{C}$-vector spaces of finite dimension, say $d_{\rho, m}$. We have

$$
\mathfrak{z}_{\mathfrak{g}}(f)=\prod_{\rho, m<0}\left(1-\rho \otimes v^{m}\right)^{d_{\rho, m}}
$$

Hence to prove (a) it suffices to show that

$$
\left(1-v^{-2}\right)^{-r} \prod_{m<0}\left(1-v^{m}\right)^{d_{1, m}} \in 1+v^{-1} \mathbf{Z}\left[v^{-1}\right] .
$$

This, in turn, would follow from the inequality

(c) $\sum_{m \text { even }} d_{1, m} \geq r$.

Let $\tilde{\mathfrak{g}}$ be the centralizer of $C$ in $\mathfrak{g}$. Then $e$ is a "distinguished" nilpotent element of $\tilde{\mathfrak{g}}$, hence as is well known, $e$ is an "even" nilpotent element of $\tilde{\mathfrak{g}}$. In other words, $\tilde{\mathfrak{g}} \cap \mathfrak{g}_{m} \neq 0$ implies that $m$ is even. Thus, in (c) the condition that $m$ is even is automatic, so it can be dropped, and then (c) just states that $\operatorname{dim}(\tilde{\mathfrak{g}} \cap \mathfrak{z} \mathfrak{g}(f)) \geq r$. Now $\tilde{\mathfrak{g}}$ is a reductive Lie algebra of rank $r$ hence, as is well known, the centralizer in $\tilde{\mathfrak{g}}$ of any element in $\tilde{\mathfrak{g}}$ (for example $f$ ) has dimension at least $r$. This proves (a).

We prove (b). We can find a Lie algebra involution $\varpi: \mathfrak{g} \rightarrow \mathfrak{g}$ such that $\varpi(e)=-e, \varpi(h)=h, \varpi(f)=-f$ and $\varpi=-1$ on the Lie algebra of $C$ (this can be easily deduced from $5.1(\mathrm{a}))$. Then $\varpi$ defines an isomorphism of the $H$-module $\mathfrak{z} \mathfrak{g}(f)$ with the $H$-module $\mathfrak{z}_{\mathfrak{g}}(f)^{\dagger}$. This proves (b).

3.3. Let $\pi: \Lambda_{e}^{\prime} \rightarrow \mathfrak{t}$ be the morphism which sends $(y, \mathfrak{b}) \in \Lambda_{e}^{\prime}$ to the image of $y$ in $\mathfrak{b} / \mathfrak{n}_{\mathfrak{b}}=\mathfrak{t}$. Note that $\pi$ is $H$-equivariant and that $\Lambda_{e}=\pi^{-1}(0)$. Consider the direct image maps

$$
K_{H}\left(\mathcal{B}_{e}\right) \stackrel{j_{*}}{\longrightarrow} K_{H}\left(\Lambda_{e}\right) \stackrel{j_{*}^{\prime}}{\longrightarrow} K_{H}\left(\Lambda_{e}^{\prime}\right) .
$$

where $j: \mathcal{B}_{e} \rightarrow \Lambda_{e}, j^{\prime}: \Lambda_{e} \rightarrow \Lambda_{e}^{\prime}$ are the imbeddings.

Lemma 3.4. (a) $j_{*}^{\prime} j_{*} \mathfrak{O}_{\mathcal{B}_{e}}=\mathfrak{z}_{\mathfrak{g}}(f)^{*} \mathfrak{O}_{\Lambda_{e}^{\prime}}$ in $K_{H}\left(\Lambda_{e}^{\prime}\right)$.

(b) $j_{*}^{\prime} \mathfrak{O}_{\Lambda_{e}}=\mathfrak{t}^{*} \mathfrak{O}_{\Lambda_{e}^{\prime}}$ in $K_{H}\left(\Lambda_{e}^{\prime}\right)$.

We prove (a). Let $E^{t}$ be the $t$-th exterior power of $\mathfrak{z}_{\mathfrak{g}}(f)^{*}$, regarded as an $H$-equivariant vector bundle on $\Lambda_{e}^{\prime}$. We have a canonical map of vector bundles $E^{t+1} \rightarrow E^{t}$ which at $(y, \mathfrak{b}) \in \Lambda_{e}^{\prime}$ is interior product by $y-e \in \mathfrak{z}_{\mathfrak{g}}(f)$. Now $\cdots \rightarrow$ $E^{2} \rightarrow E^{1} \rightarrow E^{0}$ is a free resolution of $j_{*}^{\prime} j_{*} \mathfrak{O}_{\mathcal{B}_{e}}$ and gives

$$
j_{*}^{\prime} j_{*} \mathfrak{O}_{\mathcal{B}_{e}}=\sum_{t}(-1)^{t} E^{t}=\mathfrak{z}_{\mathfrak{g}}(f)^{*} \mathfrak{O}_{\Lambda_{e}^{\prime}} \in K_{H}\left(\Lambda_{e}^{\prime}\right)
$$

(a) is proved.

We prove (b). Let $E^{\prime t}$ be the vector bundle on $\Lambda_{e}^{\prime}$ whose fibre at $(y, \mathfrak{b})$ is the dual of the $t$-th exterior power of $\mathfrak{b} / \mathfrak{n}_{\mathfrak{b}}$, regarded as an $H$-equivariant vector bundle on $\Lambda_{e}^{\prime}$. We have a canonical map of vector bundles $E^{\prime t+1} \rightarrow E^{\prime t}$ which at $(y, \mathfrak{b}) \in \Lambda_{e}^{\prime}$ is interior product by the image of $y$ in $\mathfrak{b} / \mathfrak{n}_{\mathfrak{b}}$. Now $\cdots \rightarrow E^{\prime 2} \rightarrow E^{\prime 1} \rightarrow E^{\prime 0}$ is a free resolution of $j_{*}^{\prime} \mathfrak{O}_{\Lambda_{e}}$ and gives

$$
j_{*}^{\prime} \mathfrak{O}_{\Lambda_{e}}=\sum_{t}(-1)^{t} E^{\prime t}=\mathfrak{t}^{*} \mathfrak{O}_{\Lambda_{e}^{\prime}} \in K_{H}\left(\Lambda_{e}^{\prime}\right) .
$$

(b) is proved. 
Theorem 3.5. $\nabla_{e} K_{H}\left(\Lambda_{e}\right) \subset j_{*} K_{H}\left(\mathcal{B}_{e}\right)$.

First we show that $j_{*}^{\prime}$ is injective. Since $K_{H}\left(\Lambda_{e}\right)$ is free as an $R_{H}$-module, the canonical map $K_{H}\left(\Lambda_{e}\right) \rightarrow K_{H}\left(\Lambda_{e}\right)_{\mathfrak{R}}$ is injective. Since $H$ has the same fixed point set on $\Lambda_{e}$ as on $\Lambda_{e}^{\prime}$, we see that $j_{*}^{\prime}$ induces an isomorphism $K_{H}\left(\Lambda_{e}\right)_{\mathfrak{R}} \stackrel{\sim}{\rightarrow} K_{H}\left(\Lambda_{e}^{\prime}\right)_{\mathfrak{R}}$ (see $[\mathrm{Th}]$ ). The injectivity of $j_{*}^{\prime}$ follows.

Using 3.4 we have

$$
\mathfrak{t}^{*} j_{*}^{\prime} j_{*} \mathfrak{O}_{\mathcal{B}_{e}}=\mathfrak{t}^{*} \cdot \mathfrak{z}_{\mathfrak{g}}(f)^{*} \mathfrak{O}_{\Lambda_{e}^{\prime}}=\mathfrak{z}_{\mathfrak{g}}(f)^{*} j_{*}^{\prime}\left(\mathfrak{O}_{\Lambda_{e}}\right) .
$$

Hence

$$
j_{*}^{\prime}\left(\mathfrak{t}^{*} j_{*} \mathfrak{O}_{\mathcal{B}_{e}}-\mathfrak{z}_{\mathfrak{g}}(f)^{*} \mathfrak{O}_{\Lambda_{e}}\right)=0
$$

Using the injectivity of $j_{*}^{\prime}$, we deduce

$$
\mathfrak{t}^{*} j_{*} \mathfrak{O}_{\mathcal{B}_{e}}=\mathfrak{z}_{\mathfrak{g}}(f)^{*} \mathfrak{O}_{\Lambda_{e}} .
$$

If $E \in \operatorname{Vec}_{H}\left(\Lambda_{e}\right)$, then $\mathfrak{z}_{\mathfrak{g}}(f)^{*} E=\mathfrak{t}^{*} j_{*} \mathcal{O}_{\mathcal{B}_{e}} \otimes E=\mathfrak{t}^{*} j_{*}\left(\left.E\right|_{\mathcal{B}_{e}}\right)$. Now the elements $E$ generate $K_{H}\left(\Lambda_{e}\right)$ as an abelian group, since $\Lambda_{e}$ is smooth. Hence

$$
\mathfrak{z}_{\mathfrak{g}}(f)^{*} K_{H}\left(\Lambda_{e}\right) \subset \mathfrak{t}^{*} j_{*} K_{H}\left(\mathcal{B}_{e}\right)
$$

By 3.1(a), $\mathfrak{z}_{\mathfrak{g}}(f)^{*}$ is equal to $\mathfrak{z}_{\mathfrak{g}}(f)$ times an invertible element of $R_{H}$. Similarly, $\mathfrak{t}^{*}=(-1)^{r} v^{2 r}[\mathfrak{t}$. It follows that

$$
\mathfrak{z} \mathfrak{g}(f) K_{H}\left(\Lambda_{e}\right) \subset \mathfrak{t} j_{*} K_{H}\left(\mathcal{B}_{e}\right) .
$$

Hence $\mathfrak{t} \nabla_{e} K_{H}\left(\Lambda_{e}\right) \subset \mathfrak{t} j_{*} K_{H}\left(\mathcal{B}_{e}\right)$. Since $K_{H}\left(\Lambda_{e}\right)$ is a free $R_{H}$-module, we can cancel $\mathfrak{t}$. The theorem is proved.

3.6. Let $K_{H}^{\prime}\left(\mathcal{B}_{e}\right)$ be the Grothendieck group of the category of $H$-equivariant vector bundles on $\mathcal{B}_{e}$. Consider the diagram

$$
K_{H}\left(\Lambda_{e}\right) \stackrel{a}{\rightarrow} K_{H}^{\prime}\left(\mathcal{B}_{e}\right) \stackrel{b}{\rightarrow} K_{H}\left(\mathcal{B}_{e}\right) \stackrel{j_{*}}{\rightarrow} K_{H}\left(\Lambda_{e}\right)
$$

( $a$ is given by restricting vector bundles; (b) is the obvious map). The following strengthening of 3.5 holds:

$$
\nabla_{e} K_{H}\left(\Lambda_{e}\right) \subset j_{*} b K_{H}^{\prime}\left(\mathcal{B}_{e}\right) .
$$

Indeed, the proof of 3.5 shows that for any $E \in \operatorname{Vec}_{H}\left(\Lambda_{e}\right)$, the elements $\nabla_{e} E$ and $j_{*} b a(E)$ are equal up to an invertible element of $R_{H}$.

\section{4. $\mathcal{H}$-Module STRUCTURES}

4.1. In this section we define $\mathcal{H}$-module structures on $K_{H}\left(\mathcal{B}_{e}\right), K_{H}\left(\Lambda_{e}\right)$ by reduction to the case where $G$ is simply connected which is treated in [L5]. (See [L5, 0.2, $0.5,7.9]$ for historical remarks and references to original sources.) 
4.2. Let $i \in I$ and let $\mathcal{O}_{\sigma_{i}} \subset \mathcal{B} \times \mathcal{B}$ be the corresponding $G$-orbit (see [L5, 7.3]); let $\overline{\mathcal{O}}_{i}$ be the closure of $\mathcal{O}_{\sigma_{i}}$ in $\mathcal{B} \times \mathcal{B}$. Let $Z_{i}$ be the subvariety of $\Lambda \times \Lambda$ consisting of all $\left(y, \mathfrak{b}, y, \mathfrak{b}^{\prime}\right)$ such that $\left(\mathfrak{b}, \mathfrak{b}^{\prime}\right) \in \mathcal{O}_{\sigma_{i}}$. As in [L5, 7.16], let $\bar{Z}_{i}$ be the subvariety of $\Lambda \times \Lambda$ consisting of all $\left(y, \mathfrak{b}, y, \mathfrak{b}^{\prime}\right)$ such that $\left(\mathfrak{b}, \mathfrak{b}^{\prime}\right) \in \overline{\mathcal{O}}_{i}$ and $y$ belongs to $\mathfrak{n}_{\mathfrak{p}}$ where $\mathfrak{p}$ is the unique parabolic subalgebra of type $i$ containing $\mathfrak{b}, \mathfrak{b}^{\prime}$.

Let $L^{\prime} \otimes L^{\prime \prime} \in \operatorname{Vec}_{G}\left(\overline{\mathcal{O}}_{i}\right)$ be defined as in [L5, 7.19]. Here $L^{\prime}, L^{\prime \prime}$ are line bundles on $\mathcal{B}$ that are only equivariant under the simply connected covering of the derived group of $G$, but $L^{\prime} \otimes L^{\prime \prime}$ is actually equivariant under the adjoint group of $G$ and we regard it as a $G$-equivariant line bundle with trivial action of the centre of $G$. We also regard it as an object of $\operatorname{Vec}_{G \times \mathbf{C}^{*}}\left(\overline{\mathcal{O}}_{i}\right)$ with trivial action of $\mathbf{C}^{*}$ or as an object of $\operatorname{Vec}_{H}\left(\overline{\mathcal{O}}_{i}\right)$ (via our imbedding $H \subset G \times \mathbf{C}^{*}$ ). The inverse image of this line bundle under the canonical map $\bar{Z}_{i} \rightarrow \overline{\mathcal{O}}_{i}$ is denoted again by $L^{\prime} \otimes L^{\prime \prime} \in \operatorname{Vec}_{H}\left(\bar{Z}_{i}\right)$.

4.3. Let $\mathcal{Y}$ be an $H$-stable closed subvariety of $\Lambda$. As in [L5, 10.22], we say that $\mathcal{Y}$ is $i$-saturated if the following holds:

$$
\left(y, \mathfrak{b}^{\prime}\right) \in \mathcal{Y},(y, \mathfrak{b}) \in \Lambda,\left(\mathfrak{b}, \mathfrak{b}^{\prime}\right) \in \mathcal{O}_{\sigma_{i}} \Longrightarrow(y, \mathfrak{b}) \in \mathcal{Y} .
$$

For such $\mathcal{Y}$, we define $r_{i}: K_{H}(\mathcal{Y}) \rightarrow K_{H}(\mathcal{Y})$ by

$$
r_{i}(F)=q_{*}\left(v^{-1}\left(L^{\prime} \otimes L^{\prime \prime}\right) \otimes^{L}(\mathbf{C} \otimes F)\right)
$$

where $\otimes^{L}$ (see $\left.[\mathrm{L} 5,6.4]\right)$ is computed in the smooth manifold $\Lambda^{2}$ relative to the closed subvarieties $\bar{Z}_{i}$ and $\Lambda \times \mathcal{Y}$ with intersection

$$
\mathfrak{B}=\left\{\left(y, \mathfrak{b}, y, \mathfrak{b}^{\prime}\right) \in \bar{Z}_{i} \mid\left(y, \mathfrak{b}^{\prime}\right) \in \mathcal{Y}\right\}
$$

here, $q: \mathfrak{B} \rightarrow \mathcal{Y}$ is given by $q\left(y, \mathfrak{b}, y, \mathfrak{b}^{\prime}\right)=(y, \mathfrak{b})$. (Note that $q$ is well defined since $\mathcal{Y}$ is $i$-saturated.) Using the definition and [L5, 6.5] we can check the following.

(a) If $\mathcal{Y}^{\prime} \subset \mathcal{Y}$ are two $H$-stable $i$-saturated closed subsets of $\Lambda$, then the maps $r_{i}: K_{H}(\mathcal{Y}) \rightarrow K_{H}(\mathcal{Y}), r_{i}: K_{H}\left(\mathcal{Y}^{\prime}\right) \rightarrow K_{H}\left(\mathcal{Y}^{\prime}\right)$ are compatible with the direct image map $K_{H}(\mathcal{Y}) \rightarrow K_{H}\left(\mathcal{Y}^{\prime}\right)$ induced by the inclusion $\mathcal{Y}^{\prime} \subset \mathcal{Y}$.

Proposition 4.4. (a) There is a unique $\mathcal{H}$-module structure on $K_{H}\left(\mathcal{B}_{e}\right)$ in which $v \in \mathcal{H}$ acts as $v \in R_{H},-\tilde{T}_{\sigma_{i}}-v^{-1} \in \mathcal{H}$ acts as $r_{i}: K_{H}\left(\mathcal{B}_{e}\right) \rightarrow K_{H}\left(\mathcal{B}_{e}\right)$ for any $i \in I$ and $\theta_{x}(x \in \mathcal{X})$ acts as tensor product with $L_{x}$ (regarded as an object of $\operatorname{Vec}_{G \times \mathbf{C}^{*}}(\mathcal{B})$ with trivial action of $\mathbf{C}^{*}$, or via $\mathcal{B}_{e} \rightarrow \mathcal{B}, H \subset G \times \mathbf{C}^{*}$, as an object of $\left.\operatorname{Vec}_{H}\left(\mathcal{B}_{e}\right)\right)$. The $\mathcal{H}$-module structure commutes with the $R_{H}$-module structure.

(b) The same holds if in (a) we replace $\mathcal{B}_{e}$ by $\Lambda_{e}$.

(c) The canonical imbedding $K_{H}\left(\mathcal{B}_{e}\right) \rightarrow K_{H}\left(\Lambda_{e}\right)$ (direct image under $\mathcal{B}_{e} \subset \Lambda_{e}$ ) is $\mathcal{H}$-linear.

We prove (a). In the case where $G$ is simply connected, this follows from [L5, 10.1, 7.25]. The same argument applies more generally to the case where $G$ has simply connected derived group. For general $G$ we can find a surjective homomorphism $\tilde{G} \rightarrow G$ with central kernel where $\tilde{G}$ is connected reductive with simply connected derived group. Using 1.17, the fact that $K_{H}\left(\mathcal{B}_{e}\right)$ is a free $R_{H}$-module (see 1.14) and the fact that the canonical map $R_{H} \rightarrow R_{\tilde{H}}$ is injective (where $\tilde{H}$ is as in 1.16), we see that $K_{H}\left(\mathcal{B}_{e}\right)$ is naturally imbedded in $K_{\tilde{H}}\left(\mathcal{B}_{e}\right)$. Hence the necessary relations between the generators of $\mathcal{H}$ hold on $K_{H}\left(\mathcal{B}_{e}\right)$ since they hold on the larger space $K_{\tilde{H}}\left(\mathcal{B}_{e}\right)$. This proves (a). The proof of (b) is entirely similar. Now (c) follows immediately from $4.3(\mathrm{a})$. The proposition is proved. 
4.5. From 4.4 we deduce that there is a unique $\mathcal{H}$-module structure on the $\mathfrak{R}$ vector space $\mathfrak{E}_{e}$ whose restriction to $K_{H}\left(\mathcal{B}_{e}\right), K_{H}\left(\Lambda_{e}\right)$ is as in $4.4(\mathrm{a}),(\mathrm{b})$ and which commutes with the $\mathfrak{R}$-module structure. For any $\xi, \xi^{\prime} \in \mathfrak{E}_{e}$ we have

$$
\begin{gathered}
\left(\tilde{T}_{w} \xi: \xi^{\prime}\right)=\left(\xi: \tilde{T}_{w^{-1}} \xi^{\prime}\right), \\
\left(\theta_{x} \xi: \xi^{\prime}\right)=\left(\xi: \theta_{x} \xi^{\prime}\right),
\end{gathered}
$$

where $w \in W$ and $x \in \mathcal{X}$. (See [L5, 12.12].)

Theorem 4.6. Fix $i \in I$. We have $\tilde{T}_{\sigma_{i}} \mathfrak{O}_{\Lambda_{e}}=-v^{-1} \mathfrak{O}_{\Lambda_{e}}$ in $K_{H}\left(\Lambda_{e}\right)$.

The proof will be given in 4.9 .

4.7. For a parabolic subalgebra $\mathfrak{p}$ of $\mathfrak{g}$ we denote by $\mathfrak{r}_{\mathfrak{p}}$ the solvable radical of $\mathfrak{p}$.

Let $\mathcal{Z}_{i}$ be the variety of all quadruples $\left(y, \mathfrak{b}, y, \mathfrak{b}^{\prime}\right) \in \mathfrak{g} \times \mathcal{B} \times \mathfrak{g} \times \mathcal{B}$ such that, if $\mathfrak{p}$ (resp. $\mathfrak{p}^{\prime}$ ) is the unique parabolic of type $i$ containing $\mathfrak{b}\left(\right.$ resp. $\mathfrak{b}^{\prime}$ ), then $\mathfrak{p}=\mathfrak{p}^{\prime}$ and $y \in \mathfrak{r}_{\mathfrak{p}}$.

(This implies $y \in \mathfrak{b} \cap \mathfrak{b}^{\prime}$ since $\mathfrak{r}_{\mathfrak{p}} \subset \mathfrak{b} \cap \mathfrak{b}^{\prime}$.) Recall that $\mathfrak{t}$ is the abstract Cartan algebra of $\mathfrak{g}$. Let $r: \mathcal{Z}_{i} \rightarrow \mathfrak{t}$ be the morphism which sends $\left(y, \mathfrak{b}, y, \mathfrak{b}^{\prime}\right)$ to the image of $y$ in $\mathfrak{b} / \mathfrak{n}_{\mathfrak{b}}=\mathfrak{b}^{\prime} / \mathfrak{n}_{\mathfrak{b}^{\prime}}=\mathfrak{t}$. The image of $r$ is actually a hyperplane $\mathfrak{t}_{i}$ in $\mathfrak{t}$. (This is the fixed point set of the simple reflection in $\mathfrak{t}$ determined by $i$.) Hence $r$ defines a morphism $r^{\prime}: \mathcal{Z}_{i} \rightarrow \mathfrak{t}_{i}$. A standard argument shows that

(a) $r^{\prime}$ is smooth.

Note that $\mathcal{Z}_{i}$ is smooth, connected of dimension $2 \nu+\operatorname{dim} \mathfrak{t}-1$. We have $r^{\prime-1}(0)=\bar{Z}_{i}$. It is smooth, connected of dimension $2 \nu$.

Lemma 4.8. The variety $\left\{\left(y, \mathfrak{b}, y, \mathfrak{b}^{\prime}\right) \in \mathcal{Z}_{i} \mid y \in \Sigma_{e}\right\}$ is smooth of pure dimension $\operatorname{dim} \Lambda_{e}$.

Let $\mathcal{Z}_{i, \Sigma_{e}^{\prime}}=\left\{\left(y, \mathfrak{b}, y, \mathfrak{b}^{\prime}\right) \in \mathcal{Z}_{i} \mid y \in \Sigma_{e}^{\prime}\right\}$. We have a cartesian diagram



where the horizontal maps are given by the $G$-action and the vertical maps are the obvious projections. The lower horizontal map is known to be smooth $[\mathrm{S}]$ with fibres of pure dimension $\operatorname{dim} \Sigma_{e}^{\prime}$. Hence the upper horizontal map is smooth with fibres of pure dimension $\operatorname{dim} \Sigma_{e}^{\prime}$. Hence the composition

$$
G \times \mathcal{Z}_{i, \Sigma_{e}^{\prime}} \rightarrow \mathcal{Z}_{i} \stackrel{r^{\prime}}{\longrightarrow} \mathfrak{t}_{i}
$$

is smooth with fibres of pure dimension $\operatorname{dim} \Sigma_{e}^{\prime}+2 \nu$. (See 4.7(a).) This is the same as the composition $G \times \mathcal{Z}_{i, \Sigma_{e}^{\prime}} \stackrel{p r_{2}}{\longrightarrow} \mathcal{Z}_{i, \Sigma_{e}^{\prime}} \stackrel{r^{\prime \prime}}{\longrightarrow} \mathfrak{t}_{i}$ where $r^{\prime \prime}$ is the restriction of $r^{\prime}$. It follows that $\mathcal{Z}_{i, \Sigma_{e}^{\prime}} \stackrel{r^{\prime \prime}}{\longrightarrow} \mathfrak{t}_{i}$ is smooth with fibres of pure dimension

$$
\operatorname{dim} \Sigma_{e}^{\prime}+2 \nu-\operatorname{dim} G=\operatorname{dim} \Sigma_{e}^{\prime}-\operatorname{dim} \mathfrak{t}=\operatorname{dim} \Lambda_{e} .
$$

In particular, $r^{\prime \prime-1}(0)=\left\{\left(y, \mathfrak{b}, y, \mathfrak{b}^{\prime}\right) \in \mathcal{Z}_{i} \mid y \in \Sigma_{e}\right\}$ is smooth of pure dimension $\operatorname{dim} \Lambda_{e}$. 
4.9. Proof of Theorem 4.6. The smooth subvarieties $\bar{Z}_{i}, \Lambda \times \Lambda_{e}$ of $\Lambda^{2}$ (of dimension $\left.2 \nu, 2 \nu+\operatorname{dim} \Lambda_{e}\right)$ intersect transversally in $\left\{\left(y, \mathfrak{b}, y, \mathfrak{b}^{\prime}\right) \in \mathcal{Z}_{i} \mid y \in \Sigma_{e}\right\}$, a smooth submanifold of dimension $\operatorname{dim} \Lambda_{e}$. (See Lemma 4.8.) Hence the Tor-product $v^{-1}\left(L^{\prime} \otimes L^{\prime \prime}\right) \otimes_{\Lambda^{2}}^{L}(\mathbf{C} \otimes \mathbf{C})$ (notation of $[L 5,6.4]$ ), computed in $\Lambda^{2}$ relative to its subvarieties $\bar{Z}_{i}, \Lambda \times \Lambda_{e}$, is just the vector bundle obtained by restricting the vector bundles $v^{-1}\left(L^{\prime} \otimes L^{\prime \prime}\right), \mathbf{C} \otimes \mathbf{C}$ to the intersection and then taking the usual tensor product. We obtain the vector bundle $v^{-1}\left(L^{\prime} \otimes L^{\prime \prime}\right)$ on $\left\{\left(y, \mathfrak{b}, y, \mathfrak{b}^{\prime}\right) \in \mathcal{Z}_{i} \mid y \in \Sigma\right\}$. We now map the last space to $\Lambda_{e}$ by $q:\left(y, \mathfrak{b}, y, \mathfrak{b}^{\prime}\right) \mapsto(y, \mathfrak{b})$. Then

$$
q_{*}\left(v^{-1}\left(L^{\prime} \otimes L^{\prime \prime}\right)\right)=r_{i}\left(\mathfrak{O}_{\Lambda_{e}}\right) .
$$

Let $\Lambda_{e, i}$ be the subvariety of $\Lambda_{e}$ consisting of those $(y, \mathfrak{b})$ such that $y$ is contained in the nil-radical of the unique parabolic of type $i$ that contains $\mathfrak{b}$. Note that $q$ factors as

$$
\left\{\left(y, \mathfrak{b}, y, \mathfrak{b}^{\prime}\right) \in \mathcal{Z}_{i} \mid y \in \Sigma_{e}\right\} \stackrel{q^{\prime}}{\longrightarrow} \Lambda_{e, i} \rightarrow \Lambda_{e}
$$

where the last map is the inclusion. We want to show that $r_{i}\left(\mathfrak{O}_{\Lambda_{e}}\right)=0$. It is enough to show that $q_{*}^{\prime}\left(v^{-1}\left(L^{\prime} \otimes L^{\prime \prime}\right)\right)=0$. Now $q^{\prime}$ is a $P^{1}$-bundle. Indeed, if $(y, \mathfrak{b}) \in \Lambda_{e, i}$, then the fibre of $q^{\prime}$ at $(y, \mathfrak{b})$ is isomorphic to the space of all $\mathfrak{b}^{\prime}$ contained in the unique parabolic of type $i$ that contains $\mathfrak{b}$. Hence to show that $q_{*}^{\prime}\left(v^{-1}\left(L^{\prime} \otimes L^{\prime \prime}\right)\right)=0$ it is enough to show that the cohomology of any fibre of $q^{\prime}$ with coefficients in the restriction of $v^{-1}\left(L^{\prime} \otimes L^{\prime \prime}\right)$ is zero. But this restriction is a square root of the cotangent bundle of $P^{1}$. The theorem follows.

$$
\text { 5. The SETS } \mathbf{B}_{\mathcal{B}_{e}}^{ \pm}, \mathbf{B}_{\Lambda_{e}}^{ \pm}
$$

5.1. Recall that $(e, h, f)$ has been fixed in 1.8. Let $I_{e}$ be the set of irreducible components of $\mathcal{B}_{e}$. Recall that (by Spaltenstein) all irreducible components of $\mathcal{B}_{e}$ have the same dimension, say, $b(e)$. The homology space $H_{2 b(e)}\left(\mathcal{B}_{e}, \mathbf{C}\right)$ has therefore a natural basis indexed by $I_{e}$ and so it can be identified naturally with the abstract vector space $\mathbf{C}\left[I_{e}\right]$ with basis indexed by $I_{e}$. According to Springer, $W$ acts naturally on $H_{2 b(e)}\left(\mathcal{B}_{e}, \mathbf{C}\right)$. Hence $W$ acts naturally on $\mathbf{C}\left[I_{e}\right]$. We shall be particularly interested in the action of $w_{0}$, the longest element of $W$, on $\mathbf{C}\left[I_{e}\right]$.

We now make the assumption (until the end of 5.5) that $(e, h, f)$ is distinguished in $\mathfrak{g}$, that is, $y \in \mathfrak{g},[y, e]=[y, h]=[y, f]=0 \Longrightarrow y \in \operatorname{centre}(\mathfrak{g})$. Let $\tilde{A}$ be the group of all automorphisms of the Lie algebra $\mathfrak{g}$ which keep each of $e, h, f$ fixed and induce the identity on the centre of $\mathfrak{g}$. This group is finite. Let $A$ be the intersection of $\tilde{A}$ with the group of inner automorphisms of $\mathfrak{g}$. Let $A^{1}$ be the intersection of $\tilde{A}$ with the set of automorphisms of $\mathfrak{g}$ which induce the automorphism $w \mapsto w_{0} w w_{0}^{-1}$ of $W$. (We identify $W$ with the "abstract" Weyl group of $\mathfrak{g}$ in the standard way; then any automorphism of the Lie algebra $\mathfrak{g}$ induces an automorphism of $W$.) The group $\tilde{A}$ acts naturally on $I_{e}$ hence on $\mathbf{C}\left[I_{e}\right]$. We have

(a) $A^{1} \neq \emptyset$.

This can be proved as follows. By a result of Sekiguchi [Se] (conjectured by Kostant) the following result is equivalent to (a):

(b) if $\mathcal{O}$ is a distinguished nilpotent $G$-orbit in a real split form of $\mathfrak{g}$, then $\mathcal{O}$ has at least one real point.

By Bala-Carter, we can find a parabolic subalgebra $\mathfrak{p}$ of $\mathfrak{g}$ with nil-radical $\mathfrak{n}$ such that $\mathcal{O} \cap \mathfrak{n}$ is open dense in $\mathfrak{n}$. We may assume that $\mathfrak{p}$ is defined over $\mathbf{R}$. Then $\mathfrak{n}$ is defined over $\mathbf{R}$. Hence the set of real points of $\mathfrak{n}$ is Zariski dense in $\mathfrak{n}$. In particular, 
it must have non-empty intersection with the non-empty open subset $\mathcal{O} \cap \mathfrak{n}$ of $\mathfrak{n}$. A point in this intersection is a real point of $\mathcal{O}$. This proves (b) hence also (a).

(Note that (b) remains true if we drop the word "distinguished".)

Proposition 5.2. There exists a unique involution $u_{0}: I_{e} \rightarrow I_{e}$ such that the linear map $\mathbf{C}\left[I_{e}\right] \rightarrow \mathbf{C}\left[I_{e}\right]$ induced by $u_{0}$ coincides with $\pm w_{0}: \mathbf{C}\left[I_{e}\right] \rightarrow \mathbf{C}\left[I_{e}\right]$.

Moreover, if we assume that either $\mathfrak{g}$ has no factor of type $E_{8}$ or that $\mathfrak{g}$ is simple of type $E_{8}$ and $e$ is not of type $E_{8}\left(b_{6}\right)$, then there is a unique involution $a^{1} \in A^{1}$ such that $a^{1}$ induces $u_{0}: I_{e} \rightarrow I_{e}$.

(We use the notation of $[\mathrm{C}]$ for nilpotent classes in exceptional Lie algebras.)

If $T: \mathbf{C}\left[I_{e}\right] \rightarrow \mathbf{C}\left[I_{e}\right]$ commutes with the $W$-action on $\mathbf{C}\left[I_{e}\right]$ then, by the theory of Springer correspondence, we have $T=\sum_{a \in A} c_{a} a$ for some $c_{a} \in \mathbf{C}$. We apply this to $T=w_{0} a_{1}$ where $a_{1} \in A^{1}$. (Note that $T$ commutes with the $W$-action on $\mathbf{C}\left[I_{e}\right]$.) Hence we have $w_{0} a_{1}=\sum_{a \in A} c_{a} a: \mathbf{C}\left[I_{e}\right] \rightarrow \mathbf{C}\left[I_{e}\right]$ for some $c_{a} \in \mathbf{C}$. Hence we have $w_{0}=\sum_{a^{\prime} \in A^{1}} c_{a^{\prime}} a^{\prime}: \mathbf{C}\left[I_{e}\right] \rightarrow \mathbf{C}\left[I_{e}\right]$ for some $c_{a^{\prime}} \in \mathbf{C}$.

To prove the proposition we may assume that $\mathfrak{g}$ is simple. We shall consider the various cases separately.

(I) If $\mathfrak{g}$ has type $A_{n}$, the result is trivial: $I_{e}$ has a single element and $u_{0}=1$.

(II) Assume now that $\mathfrak{g}=\mathfrak{s o}(V)$ where $V$ is an $N$-dimensional vector space with a non-singular symmetric bilinear form and $N=2 n+1 \geq 5$. We can write $V=V_{1} \oplus V_{2} \oplus \ldots \oplus V_{s}$ (orthogonal decomposition) where each $V_{j}$ is stable under $e, h, f$ and is a single Jordan block under $e$; we may assume that $\operatorname{dim}\left(V_{j}\right)=2 k_{j}+1$ where $0 \leq k_{1}<k_{2}<\cdots<k_{s}$. Note that $s=2 s_{1}+1$ for some integer $s_{1}$. Let $a^{1}: V \rightarrow V$ be the automorphism whose restriction to $V_{j}$ is multiplication by $(-1)^{n+k_{j}-1+j}$ for all $j$. This induces an automorphism of $\mathfrak{g}$ denoted again by $a^{1}$. We have $a^{1} \in A=A^{1}$. We have

(a) $w_{0}=\epsilon a^{1}$

as linear maps $\mathbf{C}\left[I_{e}\right] \rightarrow \mathbf{C}\left[I_{e}\right]$, where $\epsilon=(-1)^{s_{1}}(-1)^{k_{2}+k_{4}+\cdots+k_{2 s_{1}}}$.

This can be proved as follows. Let $\tilde{k}_{j}=k_{j}+j-1$. As a $W \times A$ module we have $\mathbf{C}\left[I_{e}\right]=\bigoplus_{\rho} E_{\rho} \otimes \rho$ where $\rho$ runs over all characters $A \rightarrow\{1,-1\}$ and $E_{\rho}$ is a $W$-module (irreducible or zero) which is explicitly known (see [L2, Sec. 11, 13]). More precisely, the various $\rho$ such that $E_{\rho} \neq 0$ are in bijection with the subsets $J \subset[1, s]$ such that $|J|=s_{1}$. (In this correspondence, $\rho(a)=\prod_{j \in J * J_{0}} \operatorname{det}\left(a, V_{j}\right)$ for $a \in A$; here $J_{0}=\left\{2,4, \ldots, 2 s_{1}\right\}$ and $J * J_{0}$ is the symmetric difference.) If $\rho$ corresponds to $J$, then $E_{\rho}$ is the irreducible $W$-module corresponding to the ordered pair of partitions in which the first partition has parts $\tilde{k}_{i_{p}}-2(p-1)$ where $i_{1}<i_{2}<\ldots<i_{s_{1}+1}$ are the elements of $[1, s]-J$ and the second partition has parts $\tilde{k}_{j_{p}}-2(p-1)$ where $j_{1}<j_{2}<\ldots<j_{s_{1}}$ are the elements of $J$. The action of $w_{0}$ on this representation of $W$ is by multiplication by $(-1)^{g_{J}}$ where $g_{J}=\sum_{j \in J} \tilde{k}_{j}$. Note that $g_{J}$ is defined for any subset $J$ of $[1, s]$ and it satisfies $g_{J * J^{\prime}}=g_{J}+g_{J^{\prime}}$ $\bmod 2$. We set $f_{J}=g_{J * J_{0}}$. We have $f_{J}=g_{J}+g_{J_{0}} \bmod 2$. Then $J \rightarrow(-1)^{f_{J}}$ is a homomorphism of the $\mathbf{F}_{2}$ vector space $\{J|J \subset[1, s]| J \mid,=0 \bmod 2\}$ into $\{ \pm 1\}$. This vector space is generated by the elements $J_{i}=\{J \mid J=[1, s]-\{i\}\}$ for various $i \in[1, s]$ (which satisfy a single relation: their sum is zero).

Now $A$ is naturally the dual of this $\mathbf{F}_{2}$-vector space. Also $A$ is contained in $\mathbf{F}_{2}^{s}$ (with natural basis $a_{i}, i \in[1, s]$ ); more precisely, $A$ is the set of elements in $\mathbf{F}_{2}^{s}$ whose sum of coordinates is 0 . The basis $a_{i}$ is on the one hand in natural bijection with the Jordan blocks of $e$ and on the other hand is in duality with the "basis" 
$\left(J_{i}\right)$. We have $g_{[1, s]-\{i\}}=n-\tilde{k}_{i}$. Hence, if we set $a=\sum_{I \in[1, s]}\left(n-\tilde{k}_{i}\right) a_{i} \in A$, then $a$ acts on the irreducible representation of $A$ corresponding to $J * J_{0}$ as $(-1)^{f_{J}}$; this is $(-1)^{g_{J_{0}}}$ times the action of $w_{0}$ on the corresponding representation of $W$ (if it is non-zero). In particular we have $a=a^{1}$. This proves (a) since

$$
g_{J_{0}}=s_{1}+k_{2}+k_{4}+\cdots+k_{2 s_{1}} \bmod 2 .
$$

The proposition follows in this case. Note also that in our case we have $\epsilon=(-1)^{b(e)}$.

The proof for type $D_{n}$ is similar. Assume that $\mathfrak{g}=\mathfrak{s o}(V)$ where $V$ is an $N$ dimensional vector space with a non-singular symmetric bilinear form and $N=$ $2 n \geq 8$.

We can write $V=V_{1} \oplus V_{2} \oplus \ldots \oplus V_{s}$ (orthogonal decomposition) where each $V_{j}$ is stable under $e, h, f$ and is a single Jordan block under $e$; we may assume that $\operatorname{dim}\left(V_{j}\right)=2 k_{j}+1$ where $0 \leq k_{1}<k_{2}<\cdots<k_{s}$. Note that $s=2 s_{1}$ for some integer $s_{1}$. Let $a^{1}: V \rightarrow V$ be the automorphism whose restriction to $V_{j}$ is multiplication by $(-1)^{k_{j}-1+j}$ for all $j$. This induces an automorphism of $\mathfrak{g}$ denoted again by $a^{1}$. We have $a^{1} \in A^{1}$. We again have $w_{0}=\epsilon a^{1}$ as linear maps $\mathbf{C}\left[I_{e}\right] \rightarrow \mathbf{C}\left[I_{e}\right]$, where $\epsilon= \pm 1$.

(III) Assume next that $\mathfrak{g}=\mathfrak{s p}(V)$ where $V$ is a $2 n$-dimensional vector space with a non-singular symplectic bilinear form and $n \geq 2$. We can write $V=V_{1} \oplus V_{2} \oplus \ldots \oplus$ $V_{s}$ (orthogonal decomposition) where each $V_{j}$ is stable under $e, h, f$ and is a single Jordan block under $e$; we may assume that $\operatorname{dim}\left(V_{j}\right)=2 k_{j}$ where $0 \leq k_{1}<k_{2}<$ $\cdots<k_{s}, s$ odd. Let $a^{1}: V \rightarrow V$ be the automorphism whose restriction to $V_{j}$ is multiplication by $(-1)^{k_{j}-1+j}$ for all $j$. This induces an automorphism of $\mathfrak{g}$ denoted again by $a^{1}$. We have $a^{1} \in A^{1}$. We have $w_{0}= \pm a^{1}$ as linear maps $\mathbf{C}\left[I_{e}\right] \rightarrow \mathbf{C}\left[I_{e}\right]$. The proof is similar to the orthogonal case. It is based on [L2, Sec. 11, 12]. The proposition follows in this case.

(IV) The same method (based on the explicit knowledge of the Springer correspondence; see for example [C, 13.3]) works for $\mathfrak{g}$ of type $G_{2}, F_{4}, E_{7}, E_{8}$, except in the case where $e$ is of type $E_{8}\left(b_{6}\right)$. We see that $u_{0}=1$ in the following cases: $\mathfrak{g}$ of type $G_{2} ; \mathfrak{g}$ of type $F_{4}$ and $e$ not subregular; $\mathfrak{g}$ of type $E_{7}$ and $e$ not of type $E_{7}\left(a_{3}\right)$; $\mathfrak{g}$ of type $E_{8}$ and $e$ of type

$$
E_{8}, E_{8}\left(a_{1}\right), E_{8}\left(a_{2}\right), E_{8}\left(a_{5}\right), E_{8}\left(b_{5}\right), E_{8}\left(a_{6}\right), E_{8}\left(a_{7}\right) .
$$

On the other hand $u_{0}$ is given by the action of the unique element of order 2 in $A$ in the following cases: $\mathfrak{g}$ of type $F_{4}$ and $e$ subregular; $\mathfrak{g}$ of type $E_{7}$ and $e$ of type $E_{7}\left(a_{3}\right) ; \mathfrak{g}$ of type $E_{8}$ and $e$ of type $E_{8}\left(a_{3}\right), E_{8}\left(a_{4}\right), E_{8}\left(b_{4}\right)$.

(V) Assume now that $\mathfrak{g}$ is of type $E_{6}$. If $e$ is of type $E_{6}$ or $E_{6}\left(a_{1}\right)$, then $A^{1}$ consists of a single element $a^{\prime}$. By the earlier part of the proof we must have $w_{0}=$ $c a^{\prime}: \mathbf{C}\left[I_{e}\right] \rightarrow \mathbf{C}\left[I_{e}\right]$ for some $c \in \mathbf{C}$. Since $w_{0}^{2}=1, a^{\prime 2}=1$, we must have $c= \pm 1$, hence we can take $u_{0}$ to be induced by $a^{\prime}$. If $e$ is of type $E_{6}\left(a_{3}\right)$, then $A$ has order 2 , hence $A^{1}$ consists of two elements $a_{1}^{\prime}, a_{2}^{\prime}$ both of order 2 ; we have $A=\left\{1, a_{1}^{\prime} a_{2}^{\prime}\right\}$. By the earlier part of the proof we must have $w_{0}=c_{1} a_{1}^{\prime}+c_{2} a_{2}^{\prime}: \mathbf{C}\left[I_{e}\right] \rightarrow \mathbf{C}\left[I_{e}\right]$ for some $c_{1}, c_{2} \in \mathbf{C}$. Since $w_{0}^{2}=1$, we have

$$
\left(c_{1}^{2}+c_{2}^{2}\right) 1+2 c_{1} c_{2} a_{1}^{\prime} a_{2}^{\prime}=1: \mathbf{C}\left[I_{e}\right] \rightarrow \mathbf{C}\left[I_{e}\right] .
$$

But $a_{1}^{\prime} a_{2}^{\prime}, 1$ are linearly independent as endomorphisms of $\mathbf{C}\left[I_{e}\right]$ since both characters of $A$ appear with non-zero multiplicity in $\mathbf{C}\left[I_{e}\right]$. It follows that $c_{1}^{2}+c_{2}^{2}=1$ and $2 c_{1} c_{2}=0$. Hence $\left(c_{1}, c_{2}\right)$ must be $(0, \pm 1)$ or $( \pm 1,0)$. Thus we may assume that 
$w_{0}= \pm a_{1}^{\prime}$. We can then take $u_{0}$ to be induced by $a_{1}^{\prime}$. Thus the proposition holds in this case.

(VI) Finally, assume that $\mathfrak{g}$ is of type $E_{8}$ and $e$ is of type $E_{8}\left(b_{6}\right)$. In this case $A$ may be identified with $S_{3}$, the symmetric group in 3 letters. Let $u, r, \epsilon$ denote the trivial, reflection, sign representation of $S_{3}$. As a $W \times A$ module we have $\mathbf{C}\left[I_{e}\right]=E_{u} \otimes u \oplus E_{r} \otimes r \oplus E_{\epsilon} \otimes \epsilon$. Here $E_{u}, E_{r}, E_{\epsilon}$ are irreducible $W$-modules which are explicitly known; in particular, $w_{0}$ acts on them as $1,1,-1$ respectively. By an earlier result we have

(b) $w_{0}=a+b s+b^{\prime} s^{\prime}+b^{\prime \prime} s s^{\prime} s+c s s^{\prime}+c^{\prime} s^{\prime} s$

as maps $\mathbf{C}\left[I_{e}\right] \rightarrow \mathbf{C}\left[I_{e}\right]$. Here $a, b, b^{\prime}, b^{\prime \prime}, c, c^{\prime} \in \mathbf{C}$ and $s, s^{\prime}$ are distinct elements of order 2 in $S_{3}$. Since the $W$-action commutes with the $A$-action, the right hand side of (b) must commute with the $A$-action on $\mathbf{C}\left[I_{e}\right]$. Since all irreducible representations of $A$ appear in $\mathbf{C}\left[I_{e}\right]$, it follows that the right hand side of (b) must come from a central element of the group algebra $\mathbf{C}[A]$. Hence $b=b^{\prime}=b^{\prime \prime}, c=c^{\prime}$.

Writing the equality (b) on the summand $E_{u} \otimes u$ gives $1=a+3 b+2 c$. Writing the equality (b) on the summand $E_{r} \otimes r$ gives $1=a+0 b-c$. Writing the equality (b) on the summand $E_{\epsilon} \otimes \epsilon$ gives $-1=a-3 b+2 c$. Thus we have a system of three linear equations for $a, b, c$. It gives $a=2 / 3, b=1 / 3, c=-1 / 3$. Thus we have $w_{0}=T$ on $\mathbf{C}\left[I_{e}\right]$ where $T=\left(2+s+s^{\prime}+s s^{\prime} s-s s^{\prime}-s^{\prime} s\right) / 3 \in \mathbf{C}[A]$.

Note that $T$ acts as 1 on $u$ and $r$; it acts as -1 on $\epsilon$. Now $w_{0}$ preserves the lattice $\mathbf{Z}\left[I_{e}\right]$ (more generally, the $W$-action on the homology of $\mathcal{B}_{e}$ with complex coefficients is compatible with the integral structure; this can be deduced from the intersection cohomology definition [L2] of the Springer representations). Moreover, $T$ preserves the direct sum decomposition $\mathbf{C}\left[I_{e}\right]=\bigoplus_{O} \mathbf{C}[O]$ where $O$ runs over the $A$-orbits in $I_{e}$ and $\mathbf{C}[O]$ is the subspace of $\mathbf{C}\left[I_{e}\right]$ spanned by the elements in $O$. Hence $w_{0}=T$ maps $\mathbf{Z}[O]=\mathbf{Z}\left[I_{e}\right] \cap \mathbf{C}[O]$ into itself.

In particular there is no $A$-orbit $O$ with trivial isotropy group since $T$ would not preserve $\mathbf{Z}[O]$.

Hence $I_{e}$ is a union of $A$-orbits $O$ consisting of 1,2 or 3 elements. Consider an orbit $O$ with $|O|=1$. We have $O=\{x\}, T x=(2 x+3 x-2 x) / 3=x$.

Consider an orbit $O$ with $|O|=2$. We have $O=\left\{x, x^{\prime}\right\}$, where $s s^{\prime}(x)=$ $x, s^{\prime} s(x)=x$. We have $s(x)=x^{\prime}, s^{\prime}\left(x^{\prime}\right)=x, s s^{\prime} s(x)=x^{\prime}$. Hence $T x=\left(2 x+3 x^{\prime}-\right.$ $2 x) / 3=x^{\prime}$. Similarly $T x^{\prime}=x$.

Consider an orbit $O$ with $|O|=3$. Since $\mathbf{C}[O]$ is isomorphic to $u+r$ as an $A$-module, $T$ acts as 1 on $\mathbf{C}[O]$. We have $O=\left\{x, x^{\prime}, x^{\prime \prime}\right\}$ and $T x=x, T x^{\prime}=$ $x^{\prime}, T x^{\prime \prime}=x^{\prime \prime}$. Thus, $T$ acts on $\mathbf{C}\left[I_{e}\right]$ as a permutation of the basis $I_{e}$ : the elements in any 2-element $A$-orbit are interchanged and any other element is fixed. Since $w_{0}=T$, the proposition holds in this case. The proposition is proved.

5.3. Note that in the last case considered ( $\mathfrak{g}$ of type $E_{8}$ and $e$ of type $\left.E_{8}\left(b_{6}\right)\right)$ the permutation $u_{0}$ of $I_{e}$ is not induced by an element of $A^{1}$ (unlike all the other cases).

5.4. An involution $\varpi$ of the Lie algebra $\mathfrak{g}$ has a fixed point set of dimension equal to at least the number of positive roots of $\mathfrak{g}$. If this fixed point set has dimension equal to the number of positive roots, then $\varpi$ is called an opposition of $\mathfrak{g}$. Equivalently, an opposition of $\mathfrak{g}$ is a Lie algebra involution $\varpi: \mathfrak{g} \rightarrow \mathfrak{g}$ such that there exists a Cartan subalgebra of $\mathfrak{g}$ contained in the -1 eigenspace of $\varpi$. The set of oppositions of $\mathfrak{g}$ is non-empty and the identity component of the group of automorphisms of $\mathfrak{g}$ acts on it transitively by conjugation. 
Let $\iota=\operatorname{Ad}(\phi(\sqrt{-1})): \mathfrak{g} \rightarrow \mathfrak{g}$ where $\phi$ is as in 1.8. We have $\mathfrak{g}=\bigoplus_{m \in \mathbf{Z}} \mathfrak{g}_{m}$ where $\mathfrak{g}_{m}$ is the $m$-eigenspace of $\operatorname{ad}(h): \mathfrak{g} \rightarrow \mathfrak{g}$. Since $e, h, f$ is distinguished, we have $\mathfrak{g}_{m}=0$ for odd $m$. Now $\iota$ restricted to $\mathfrak{g}_{m}$ is multiplication by $(-1)^{m / 2}$ for any even $m$. This shows that $\iota$ is independent of the choice of $\sqrt{-1}$.

Proposition 5.5. Assume that either $\mathfrak{g}$ has no factor of type $E_{8}$ or that $\mathfrak{g}$ is simple of type $E_{8}$ and $e$ is not of type $E_{8}\left(b_{6}\right)$. Let $a^{1} \in A^{1}$ be as in 5.2. Then $\iota a^{1}=a^{1} \iota$ is an opposition of $\mathfrak{g}$.

We may assume that $\mathfrak{g}$ is simple. Assume first that $\mathfrak{g}$ is of exceptional type. Then, for any involution $a^{\prime} \in A^{1}$, the product $\iota a^{\prime}=a^{\prime} \iota$ is an opposition of $\mathfrak{g}$. This can be checked by direct computation, using the knowledge of the Dynkin diagram of $e$, or it can be deduced from the tables in [D].

Next we assume that $\mathfrak{g}$ is of type $B_{n}$ or $D_{n}$ and use the notation in the proof of 5.2 (case II).

We may assume that $G=S O(V)$. Then, on $V_{j}, \phi(\sqrt{-1}) \in G$ acts with $k_{j}+1$ eigenvalues 1 and $k_{j}$ eigenvalues $-1\left(\right.$ if $\left.k_{j}=0 \bmod 2\right)$ and with $k_{j}$ eigenvalues 1 and $k_{j}+1$ eigenvalues -1 (if $k_{j}=1 \bmod 2$ ). We regard $a^{1} \in O(V)$. Then, on $V_{j}$, $a^{1}$ acts as $(-1)^{n-1+j}$ (if $k_{j}=0 \bmod 2$ ) and acts as $(-1)^{n+j}\left(\right.$ if $\left.k_{j}=1 \bmod 2\right)$. Hence on $V_{j}, \phi(\sqrt{-1}) a^{1}=a^{1} \phi(\sqrt{-1})$ acts with

$k_{j}+1$ eigenvalues $1, k_{j}$ eigenvalues -1 if $k_{j}$ is even, $j=n-1 \bmod 2$,

$k_{j}$ eigenvalues $1, k_{j}+1$ eigenvalues -1 if $k_{j}$ is even, $j=n \bmod 2$,

$k_{j}$ eigenvalues $1, k_{j}+1$ eigenvalues -1 if $k_{j}$ is odd, $j=n \bmod 2$,

$k_{j}+1$ eigenvalues $1, k_{j}$ eigenvalues -1 if $k_{j}$ is odd, $j=n-1 \bmod 2$.

The number of eigenvalues 1 of $\phi(\sqrt{-1}) a^{1}: V \rightarrow V$ is therefore

$$
\begin{aligned}
N_{1} & =\sum_{j ; k_{j}=0} \sum_{\bmod 2 ; j=n-1}\left(k_{j}+1\right)+\sum_{j ; k_{j}=0} \sum_{\bmod 2 ; j=n} k_{j}+\sum_{j ; k_{j}=1} \sum_{\bmod 2 ; j=n \bmod 2}\left(k_{j}+1\right) \\
& =\sum_{j} k_{j}+\sharp(j ; j=n-1 \bmod 2)=(N-s) / 2+x
\end{aligned}
$$

where $x=s / 2$ if $s$ is even (hence $N=2 n) ; x=(s+1) / 2$ if $s$ is odd and $n$ is even (hence $N=2 n+1) ; x=(s-1) / 2$ if $s$ is odd and $n$ is odd (hence $N=2 n+1$ ).

Let $N_{-1}$ be the number of eigenvalues -1 of $\phi(\sqrt{-1}) a^{1}: V \rightarrow V$.

If $N$ and $s$ are odd and $n$ is even we have $N_{1}=n+1$ hence $N_{-1}=n$. If $N$ and $s$ are odd and $n$ is odd we have $N_{1}=n$ hence $N_{-1}=n+1$. If $N$ and $s$ are even we have $N_{1}=n$ hence $N_{-1}=n$. It follows that $\phi(\sqrt{-1}) a^{1}: V \rightarrow V$ induces an opposition of $\mathfrak{g}$.

Next we assume that $\mathfrak{g}$ is of type $C_{n}$ and use the notation in the proof of 5.2 (case III). We may assume that $G=S p(V)$. Then, on $V_{j}, \phi(\sqrt{-1}) \in G$ acts with $k_{j}$ eigenvalues $\sqrt{-1}$ and $k_{j}$ eigenvalues $-\sqrt{-1}$. We regard $a^{1} \in S p(V)$. Then, on $V_{j}, a^{1}$ acts as \pm 1 . Hence on $V_{j}, \phi(\sqrt{-1}) a^{1}=a^{1} \phi(\sqrt{-1})$ acts with $k_{j}$ eigenvalues $\sqrt{-1}$ and $k_{j}$ eigenvalues $-\sqrt{-1}$.

It follows that $\phi(\sqrt{-1}) a^{1}: V \rightarrow V$ has $n$ eigenvalues $\sqrt{-1}$ and $n$ eigenvalues $-\sqrt{-1}$. Then $\phi(\sqrt{-1}) a^{1}: V \rightarrow V$ induces an involution of $\mathfrak{g}$ which has exactly $n^{2}$ eigenvalues equal to 1 hence is an opposition of $\mathfrak{g}$.

A similar argument applies when $\mathfrak{g}$ is of type $A_{n}$. This completes the proof. 
5.6. We no longer assume that $(e, h, f)$ is distinguished in $\mathfrak{g}$. Let $\mathfrak{A}$ be the set of all automorphisms $g: \mathfrak{g} \stackrel{\sim}{\rightarrow} \mathfrak{g}$ such that

$g(e)=-e, g(h)=h, g(f)=-f$,

$g=-1$ on the Lie algebra of $C$,

$g$ induces on $W$ the automorphism $w \mapsto w_{0} w w_{0}^{-1}$.

Note that $g \in \mathfrak{A} \Longrightarrow g^{-1} \in \mathfrak{A}$.

If $g \in \mathfrak{A}$, then $g$ defines an automorphism $(y, \mathfrak{b}) \mapsto(-g(y), g(\mathfrak{b}))$ of $\Lambda$ which maps $\mathcal{B}_{e}$ into itself and $\Lambda_{e}$ into itself; the resulting automorphisms of $\mathcal{B}_{e}, \Lambda_{e}$ are denoted again by $g$. This automorphism of $\Lambda_{e}$ (and hence its restriction to $\mathcal{B}_{e}$ ) is compatible with the action of $H$ in the following way:

$$
g((c, \lambda)(y, \mathfrak{b}))=\left(c^{-1}, \lambda\right) g(y, \mathfrak{b}) \quad \text { for }(c, \lambda) \in H,(y, \mathfrak{b}) \in \Lambda_{e} .
$$

Hence, as in [L5, 12.6], $g$ induces automorphisms $g^{*}$ of $K_{H}\left(\mathcal{B}_{e}\right)$ and $K_{H}\left(\Lambda_{e}\right)$ which are semilinear with respect to the involution ${ }^{\dagger}: R_{H} \rightarrow R_{H}$. There is a unique automorphism $g^{*}: \mathfrak{E}_{e} \rightarrow \mathfrak{E}_{e}$, semilinear with respect to the field involution ${ }^{\dagger}: \mathfrak{R} \rightarrow$ $\mathfrak{R}$ (induced by ${ }^{\dagger}: R_{H} \rightarrow R_{H}$ ) whose restriction to $K_{H}\left(\mathcal{B}_{e}\right), K_{H}\left(\Lambda_{e}\right)$ is $g^{*}$ from the previous sentence. For $\xi, \xi^{\prime} \in \mathfrak{E}_{e}$, we have

$$
\left(\xi: g^{*} \xi^{\prime}\right)=\left(\left(g^{-1}\right)^{*} \xi: \xi^{\prime}\right)^{\dagger},
$$

as in $[\mathrm{L} 5,12.12]$.

5.7. In the remainder of this paper we assume that either

(a) $\mathfrak{g}$ is simple of type $E_{8}$ and $e$ is of type $E_{8}\left(b_{6}\right)$, or

(b) the projection of $e$ onto any simple factor of type $E_{8}$ of $\mathfrak{g}$ is not of type $E_{8}\left(b_{6}\right)$.

We define $\Upsilon: \mathfrak{E}_{e} \rightarrow \mathfrak{E}_{e}$ as follows. In case (a) we set

$$
\Upsilon=\left(2(\iota .1)^{*}+(\iota s)^{*}+\left(\iota s^{\prime}\right)^{*}+\left(\iota s s^{\prime} s\right)^{*}-\left(\iota s s^{\prime}\right)^{*}-\left(\iota s^{\prime} s\right)^{*}\right) / 3
$$

where $\iota$ is as in 5.4, and $1, s, s^{\prime}, s s^{\prime} s, s s^{\prime}, s^{\prime} s$ are the elements of $A$ as in 5.2 (VI). (For any $a \in A$ we have $\iota a \in \mathfrak{A}$.)

In case (b) we set

$$
\Upsilon=\varpi^{*}
$$

where $\varpi \in \mathfrak{A}$ is defined as follows. Let $L$ be the centralizer of $C$ (see 1.8) in $G$ and let $\mathfrak{l}$ be the Lie algebra of $L$. Then $(e, h, f)$ is distinguished in $\mathfrak{l}$. Let $\varpi_{0}: \mathfrak{l} \rightarrow \mathfrak{l}$ be the opposition $\iota a^{1}=a^{1} \iota$ of $\mathfrak{l}$ defined as in 5.5 in terms of $e, h, f$ and $\mathfrak{l}$ (instead of $\mathfrak{g})$. Note that $\varpi_{0}(x)=-x$ for all $x$ in the Lie algebra of $C$. It is easy to see that any opposition of $\mathfrak{l}$ is the restriction of an opposition of $\mathfrak{g}$. In particular, we can find an opposition $\varpi: \mathfrak{g} \rightarrow \mathfrak{g}$ such that $\left.\varpi\right|_{\mathfrak{r}}=\varpi_{0}$.

We show that $\varpi$ is uniquely determined up to replacing $\varpi$ by $\operatorname{Ad}(c) \varpi \operatorname{Ad}\left(c^{-1}\right)$ with $c \in C$ (this is an opposition with the same properties as $\varpi$ ). Indeed, let $\varpi^{\prime}$ be another opposition of $\mathfrak{g}$ such that $\left.\varpi^{\prime}\right|_{\mathfrak{r}}=\varpi_{0}$. We have $\varpi^{\prime}=\operatorname{Ad}(g) \varpi$ for some $g \in G$ such that $\operatorname{Ad}(g)$ is the identity on $\mathfrak{l}$ hence $g=z c$ where $c \in C$ and $z$ is in the centre of $G$. Replacing $g$ by $z^{-1} g$ we see that we may assume that $g=c$. Let $c_{1} \in C$ be such that $c_{1}^{2}=c$. Then $\varpi^{\prime}=\operatorname{Ad}\left(c_{1}^{2}\right) \varpi=\operatorname{Ad}\left(c_{1}\right) \varpi \operatorname{Ad}\left(c_{1}^{-1}\right)$ as claimed.

Note that $\varpi \in \mathfrak{A}$ and $\varpi^{*}$ is independent of the choice of $\varpi$.

In case (b), $\Upsilon$ restricts to maps $K_{H}\left(\mathcal{B}_{e}\right) \rightarrow K_{H}\left(\mathcal{B}_{e}\right), K_{H}\left(\Lambda_{e}\right) \rightarrow K_{H}\left(\Lambda_{e}\right)$ denoted again by $\Upsilon$. Conjecturally, the same holds also in case (a). We will assume that this is indeed the case.

It is easy to see that $\Upsilon^{2}=1$. 
5.8. Extending [L5, 12.14], we define a pairing $(\|): \mathfrak{E}_{e} \times \mathfrak{E}_{e} \rightarrow \mathfrak{R}$ by

$$
\left(\xi|| \xi^{\prime}\right)=(-v)^{\nu-2 b(e)}\left(\xi: \tilde{T}_{w_{0}} \Upsilon\left(\xi^{\prime}\right)\right)=(-v)^{\nu-2 b(e)}\left(\tilde{T}_{w_{0}} \Upsilon(\xi), \xi^{\prime}\right)^{\dagger}
$$

where $(:)$ is as in 2.6. (The second equality follows from 4.5(a), 5.6(a).)

Note that $\tilde{T}_{w_{0}} \Upsilon=\Upsilon \tilde{T}_{w_{0}}$. (This follows from the equality $\tilde{T}_{\sigma_{i}} g^{*}=g^{*} \tilde{T}_{w_{0} \sigma_{i} w_{0}^{-1}}$ valid for any $g \in \mathfrak{A}, i \in I$; in turn, the last equality follows directly from the definitions.)

The pairing (\|) is $\mathfrak{R}$-linear in the first variable and $\mathfrak{R}$-semilinear (see 5.6) in the second variable. Moreover, using 2.7, we see that it is perfect and satisfies

$$
\left(\xi \| \xi^{\prime}\right)=\left(\xi^{\prime} \| \xi\right)^{\dagger}
$$

for $\xi, \xi^{\prime} \in \mathfrak{E}_{e}$. Note that

(a) (II) restricts to a pairing $(\|): K_{H}\left(\mathcal{B}_{e}\right) \times K_{H}\left(\Lambda_{e}\right) \rightarrow R_{H}$.

5.9. $R_{H}=R_{C}\left[v, v^{-1}\right]$ is naturally a subring of $R_{C}\left(\left(v^{-1}\right)\right)$. From 3.2(a) we see that $\nabla_{e}$ is an invertible element of $R_{C}\left(\left(v^{-1}\right)\right)$. Hence $\nabla_{e}^{-1} R_{H}$ (a subset of $\mathfrak{R}$ ) can also be regarded as a subset of $R_{C}\left(\left(v^{-1}\right)\right)$.

Let $p \mapsto p^{(0)}$ be the group homomorphism $R_{C} \rightarrow \mathbf{Z}$ which takes a non-trivial character of $C$ to 0 and takes the trivial character of $C$ to 1 . Let

$$
\partial: R_{C}\left[v, v^{-1}\right] \rightarrow \mathbf{Z}\left[v, v^{-1}\right]
$$

be the group homomorphism defined by $\sum_{n} p_{n} v^{n} \rightarrow \sum_{n} p_{n}^{(0)} v^{n}$. Here $p_{n} \in R_{C}$.

Lemma 5.10. (a) For $\xi, \xi^{\prime} \in K_{H}\left(\mathcal{B}_{e}\right)$, we have $\left(\xi \| \xi^{\prime}\right) \in R_{H}$.

(b) For $\xi, \xi^{\prime} \in K_{H}\left(\Lambda_{e}\right)$, we have $\left(\xi \| \xi^{\prime}\right) \in \nabla_{e}^{-1} R_{H}$.

(a) follows from 5.8(a) since $K_{H}\left(\mathcal{B}_{e}\right) \subset K_{H}\left(\Lambda_{e}\right)$. To prove (b) it is enough to check that $\left(\nabla_{e} \xi \| \xi^{\prime}\right) \in R_{H}$. This follows from 5.8(a) since $\nabla_{e} \xi \in K_{H}\left(\mathcal{B}_{e}\right)$. (See 3.5.) The lemma is proved.

5.11. Extending $[\mathrm{L} 5,12.9]$ we define a map $\tilde{\beta}: K_{H}\left(\mathcal{B}_{e}\right) \rightarrow K_{H}\left(\mathcal{B}_{e}\right)$ by

$$
\xi^{\prime} \mapsto(-v)^{-\nu} \Upsilon \tilde{T}_{w_{0}}^{-1} D_{\mathcal{B}_{e}}\left(\xi^{\prime}\right)
$$

and a map $\beta: K_{H}\left(\Lambda_{e}\right) \rightarrow K_{H}\left(\Lambda_{e}\right)$ by

$$
\xi^{\prime} \mapsto(-v)^{-\nu+2 b(e)} \Upsilon \tilde{T}_{w_{0}}^{-1} D_{\Lambda_{e}}\left(\xi^{\prime}\right) .
$$

Here $D_{\mathcal{B}_{e}}: K_{H}\left(\mathcal{B}_{e}\right) \rightarrow K_{H}\left(\mathcal{B}_{e}\right), D_{\Lambda_{e}}: K_{H}\left(\Lambda_{e}\right) \rightarrow K_{H}\left(\Lambda_{e}\right)$ is Serre-Grothendieck duality [L5, 6.10]. Note that $\tilde{\beta}, \beta$ are not compatible with the inclusion $K_{H}\left(\mathcal{B}_{e}\right) \subset$ $K_{H}\left(\Lambda_{e}\right)$. The maps $\tilde{\beta}, \beta$ are $\mathcal{H}$-antilinear (see [L5, 2.1]) involutions. (Compare [L5, 12.10].) We define

$$
\begin{gathered}
\mathbf{B}_{\mathcal{B}_{e}}^{ \pm}=\left\{\xi \in K_{H}\left(\mathcal{B}_{e}\right) \mid \tilde{\beta}(\xi)=\xi,(\xi|| \xi) \in 1+v^{-1} R_{C}\left[v^{-1}\right]\right\}, \\
\mathbf{B}_{\Lambda_{e}}^{ \pm}=\left\{\xi \in K_{H}\left(\Lambda_{e}\right) \mid \beta(\xi)=\xi,(\xi|| \xi) \in 1+v^{-1} R_{C}\left[\left[v^{-1}\right]\right]\right\} .
\end{gathered}
$$

(Compare [L5, 12.18].) The following property is obvious:

(a) If $\xi \in \mathbf{B}_{\mathcal{B}_{e}}^{ \pm}, \xi^{\prime} \in \mathbf{B}_{\Lambda_{e}}^{ \pm}, \tau \in \hat{C}$ and $\epsilon \in\{1,-1\}$, then $\epsilon \tau \xi \in \mathbf{B}_{\mathcal{B}_{e}}^{ \pm}, \epsilon \tau \xi^{\prime} \in \mathbf{B}_{\Lambda_{e}}^{ \pm}$.

The following is a variant of [L5, 12.19, 12.23]. 
Conjecture 5.12. (a) $\mathbf{B}_{\mathcal{B}_{e}}^{ \pm}$is a signed basis of the $\mathcal{A}$-module $K_{H}\left(\mathcal{B}_{e}\right)$.

(b) $\mathbf{B}_{\Lambda_{e}}^{ \pm}$is a signed basis of the $\mathcal{A}$-module $K_{H}\left(\Lambda_{e}\right)$.

(c) For $b, b^{\prime} \in \mathbf{B}_{\mathcal{B}_{e}}^{ \pm}$such that $\pm b^{\prime} \notin \hat{C} b$, we have $\left(b \| b^{\prime}\right) \in v^{-1} R_{C}\left[v^{-1}\right]$.

(d) For $b, b^{\prime} \in \mathbf{B}_{\Lambda_{e}}^{ \pm}$such that $\pm b^{\prime} \notin \hat{C} b$, we have $\left(b \| b^{\prime}\right) \in v^{-1} R_{C}\left[\left[v^{-1}\right]\right]$.

(e) For any $b \in \mathbf{B}_{\mathcal{B}_{e}}^{ \pm}$there exists $\tilde{b} \in \mathbf{B}_{\Lambda_{e}}^{ \pm}$such that $(b \| \tilde{b})=1$ and $\left(b^{\prime} \| \tilde{b}\right)=0$ for any $b^{\prime} \in \mathbf{B}_{\mathcal{B}_{e}}^{ \pm}$such that $b^{\prime}$ is not of the form $\pm \tau b$ for some $\tau \in \hat{C}$.

(f) $\tilde{b}$ in (e) is unique and $b \mapsto \tilde{b}$ is a bijection $\mathbf{B}_{\mathcal{B}_{e}}^{ \pm} \stackrel{\sim}{\rightarrow} \mathbf{B}_{\Lambda_{e}}^{ \pm}$.

(g) Let $b, b^{\prime} \in \mathbf{B}_{\Lambda_{e}}^{ \pm}$. Then either $\partial\left(\nabla_{e} b \| b^{\prime}\right) \in \mathbf{N}\left[-v^{-1}\right]$ or $-\partial\left(\nabla_{e} b \| b^{\prime}\right) \in \mathbf{N}\left[-v^{-1}\right]$.

(h) $v^{2 b(e)} \mathfrak{O}_{\Lambda_{e}} \in \mathbf{B}_{\Lambda_{e}}^{ \pm}$.

5.13. For $\xi \in \mathbf{B}_{\mathcal{B}_{e}}^{ \pm}$we write $(\xi|| \xi)=\sum_{n \geq 0} c_{n} v^{-n}$, where $c_{n} \in R_{C}$ is 0 for $n \gg 0$ and $c_{0}=1$. We show that

$$
c_{2 b(e)}=1 \text { and } c_{n}=0 \text { for } n>2 b(e) .
$$

Note that $\beta(\xi)=v^{2 b(e)} \tilde{\beta}(\xi)$ for $\xi \in K_{H}\left(\mathcal{B}_{e}\right)$. As in [L5, 12.15], we have

$$
(\tilde{\beta}(\xi) \| \xi)=\overline{(\xi \| \beta(\xi))}
$$

where ${ }^{-}: R_{C}\left[v, v^{-1}\right] \rightarrow R_{C}\left[v, v^{-1}\right]$ is the $R_{C}$-algebra involution which takes $v$ to $v^{-1}$. It follows that $(\tilde{\beta}(\xi) \| \xi)=\overline{\left(\xi \| v^{2 b(e)} \tilde{\beta}(\xi)\right)}$. Substituting $\tilde{\beta}(\xi)=\xi$ gives $(\xi \| \xi)=$ $v^{-2 b(e)} \overline{(\xi \| \xi)}$. Hence

$$
\sum_{n \geq 0} c_{n} v^{-n}=v^{-2 b(e)} \sum_{n \geq 0} c_{n} v^{n} .
$$

Comparing coefficients and using $c_{0}=1$, we deduce (a).

5.14. We now verify that the element in $5.12(\mathrm{~h})$ satisfies at least part of the defining properties of an element of $\mathbf{B}_{\Lambda_{e}}^{ \pm}$, namely that

(a) $\beta\left(v^{2 b(e)} \mathfrak{O}_{\Lambda_{e}}\right)=v^{2 b(e)} \mathfrak{O}_{\Lambda_{e}}$.

From $[\mathrm{L} 5,6.13,11.10]$ we have

(b) $D_{\Lambda_{e}}\left(\mathfrak{O}_{\Lambda_{e}}\right)=(-1)^{2 b(e)} v^{2 b(e)} \mathfrak{O}_{\Lambda_{e}}=v^{2 b(e)} \mathfrak{O}_{\Lambda_{e}}$.

Clearly, $\Upsilon \mathfrak{O}_{\Lambda_{e}}=\mathfrak{O}_{\Lambda_{e}}$. From 4.6 we have

Hence

(c) $\tilde{T}_{w_{0}}^{-1} \mathfrak{O}_{\Lambda_{e}}=(-v)^{\nu} \mathfrak{O}_{\Lambda_{e}}$.

$$
\begin{aligned}
& \beta\left(v^{2 b(e)} \mathfrak{O}_{\Lambda_{e}}\right)=v^{-2 b(e)}(-v)^{-\nu+2 b(e)} \Upsilon \tilde{T}_{w_{0}}^{-1} D_{\Lambda_{e}}\left(\mathfrak{O}_{\Lambda_{e}}\right) \\
& =(-v)^{-\nu} v^{2 b(e)} \Upsilon \tilde{T}_{w_{0}}^{-1} \mathfrak{O}_{\Lambda_{e}}=v^{2 b(e)} \Upsilon \mathfrak{O}_{\Lambda_{e}}=v^{2 b(e)} \mathfrak{O}_{\Lambda_{e}}
\end{aligned}
$$

This proves (a).

5.15. In this subsection we will restrict the $H$ actions to the subgroup $\mathbf{C}^{*}$ as in 1.8. Let $g: R_{H} \rightarrow R_{\mathbf{C}^{*}}$ be the ring homomorphism induced by the inclusion $\mathbf{C}^{*} \subset H$. We can define

$$
\begin{aligned}
& (\|): K_{\mathbf{C}^{*}}\left(\mathcal{B}_{e}\right) \times K_{\mathbf{C}^{*}}\left(\Lambda_{e}\right) \rightarrow R_{\mathbf{C}^{*}}, \\
& (\|): K_{\mathbf{C}^{*}}\left(\Lambda_{e}\right) \times K_{\mathbf{C}^{*}}\left(\Lambda_{e}\right) \rightarrow g\left(\nabla_{e}\right)^{-1} R_{\mathbf{C}^{*}}, \\
& (\|): K_{\mathbf{C}^{*}}\left(\mathcal{B}_{e}\right) \times K_{\mathbf{C}^{*}}\left(\mathcal{B}_{e}\right) \rightarrow R_{\mathbf{C}^{*}},
\end{aligned}
$$

by the same definitions as in $2.4,5.8,5.10$ (replacing $H$ by $\mathbf{C}^{*}$ ) or equivalently by change of scalars from $R_{H}$ to $R_{\mathbf{C}^{*}}$ (using 1.19). 
We can define $\tilde{\beta}: K_{\mathbf{C}^{*}}\left(\mathcal{B}_{e}\right) \rightarrow K_{\mathbf{C}^{*}}\left(\mathcal{B}_{e}\right), \underline{\beta}: K_{\mathbf{C}^{*}}\left(\Lambda_{e}\right) \rightarrow K_{\mathbf{C}^{*}}\left(\Lambda_{e}\right)$, in the same way as $\tilde{\beta}, \beta$ in 5.11 (replacing $H$ by $\mathbf{C}^{*}$ ) or, equivalently, by change of scalars from $R_{H}$ to $R_{\mathrm{C}^{*}}$ (using 1.19). We define

$$
\begin{gathered}
\underline{\mathbf{B}}_{\mathcal{B}_{e}}^{ \pm}=\left\{\xi \in K_{\mathbf{C}^{*}}\left(\mathcal{B}_{e}\right) \mid \underline{\tilde{\beta}}(\xi)=\xi,(\xi|| \xi) \in 1+v^{-1} \mathbf{Z}\left[v^{-1}\right]\right\}, \\
\underline{\mathbf{B}}_{\Lambda_{e}}^{ \pm}=\left\{\xi \in K_{\mathbf{C}^{*}}\left(\Lambda_{e}\right) \mid \underline{\beta}(\xi)=\xi,(\xi \| \xi) \in 1+v^{-1} \mathbf{Z}\left[\left[v^{-1}\right]\right]\right\} .
\end{gathered}
$$

In the case where $C=\{1\}$ we have $\mathbf{B}_{\mathcal{B}_{e}}^{ \pm}=\underline{\mathbf{B}}_{\mathcal{B}_{e}}^{ \pm}$, and $\mathbf{B}_{\Lambda_{e}}^{ \pm}=\underline{\mathbf{B}}_{\Lambda_{e}}^{ \pm}$.

We now state a variant of Conjecture 5.12.

Conjecture 5.16. (a) $\underline{\mathbf{B}}_{\mathcal{B}_{e}}^{ \pm}$is a signed basis of the $\mathcal{A}$-module $K_{\mathbf{C}^{*}}\left(\mathcal{B}_{e}\right)$.

(b) $\underline{\mathbf{B}}_{\Lambda_{e}}^{ \pm}$is a signed basis of the $\mathcal{A}$-module $K_{\mathbf{C}^{*}}\left(\Lambda_{e}\right)$.

(c) For $b, b^{\prime} \in \underline{\mathbf{B}}_{\mathcal{B}_{e}}^{ \pm}$such that $\pm b^{\prime} \neq b$, we have $\left(b \| b^{\prime}\right) \in v^{-1} \mathbf{Z}\left[v^{-1}\right]$.

(d) For $b, b^{\prime} \in \underline{\mathbf{B}}_{\Lambda_{e}}^{ \pm}$such that $\pm b^{\prime} \neq b$, we have $\left(b \| b^{\prime}\right) \in v^{-1} \mathbf{Z}\left[\left[v^{-1}\right]\right]$.

(e) For any $b \in \underline{\mathbf{B}}_{\mathcal{B}_{e}}^{ \pm}$there exists $\tilde{b} \in \underline{\mathbf{B}}_{\Lambda_{e}}^{ \pm}$such that $(b \| \tilde{b})=1$ and $\left(b^{\prime}|| \tilde{b}\right)=0$ for any $b^{\prime} \in \underline{\mathbf{B}}_{\mathcal{B}_{e}}^{ \pm}-\{ \pm b\}$.

(f) $\tilde{b}$ in $(e)$ is unique and $b \mapsto \tilde{b}$ is a bijection $\underline{\mathbf{B}}_{\mathcal{B}_{e}}^{ \pm} \stackrel{\sim}{\rightarrow} \underline{\mathbf{B}}_{\Lambda_{e}}^{ \pm}$.

(g) Let $b, b^{\prime} \in \underline{\mathbf{B}}_{\Lambda_{e}}^{ \pm}$. Then either $\left(g\left(\nabla_{e}\right) b \| b^{\prime}\right) \in \mathbf{N}\left[-v^{-1}\right]$ or $-\left(g\left(\nabla_{e}\right) b \| b^{\prime}\right) \in$ $\mathbf{N}\left[-v^{-1}\right]$.

(h) $v^{2 b(e)} \mathfrak{O}_{\Lambda_{e}} \in \underline{\mathbf{B}}_{\Lambda_{e}}^{ \pm}$.

5.17. Clearly, the obvious map $K_{H}\left(\mathcal{B}_{e}\right) \rightarrow K_{\mathbf{C}^{*}}\left(\mathcal{B}_{e}\right)$ carries $\mathbf{B}_{\mathcal{B}_{e}}^{ \pm}$into $\underline{\mathbf{B}}_{\mathcal{B}_{e}}^{ \pm}$, and the obvious map $K_{H}\left(\Lambda_{e}\right) \rightarrow K_{\mathbf{C}^{*}}\left(\Lambda_{e}\right)$ carries $\mathbf{B}_{\Lambda_{e}}^{ \pm}$into $\underline{\mathbf{B}}_{\Lambda_{e}}^{ \pm}$.

If we assume 5.12(a) and 5.16(a) we thus get a bijection between the set of $\hat{C}$-orbits on $\mathbf{B}_{\mathcal{B}_{e}}^{ \pm}$and the set $\underline{\mathbf{B}}_{\mathcal{B}_{e}}^{ \pm}$.

5.18. Assume that $G$ is adjoint. Let

$$
G_{1}=\left\{(g, \lambda) \in \operatorname{Aut}(\mathfrak{g}) \times \mathbf{C}^{*} \mid g(e)=\lambda^{2} e, g(h)=h, g(f)=\lambda^{-2} f\right\} .
$$

The map $H \rightarrow G_{1}$ given by $(c, \lambda) \rightarrow(\operatorname{Ad}(c \phi(\lambda)), \lambda)$ identifies $H$ with a maximal torus of $G_{1}$. Let $\mathcal{N}$ be the normalizer of $H$ in $G_{1}$. Then $\mathcal{N}$ acts on $\Lambda_{e}$ by $g:(y, \mathfrak{b}) \rightarrow\left(\lambda^{-2} g(y), g(\mathfrak{b})\right)$ and $\mathcal{B}_{e}$ is stable under this action. Let $g \in \mathcal{N}$. If $F \in \operatorname{Coh}_{H}\left(\mathcal{B}_{e}\right)$, then $g^{*} F$ is naturally an object of $\operatorname{Coh}_{H}\left(\mathcal{B}_{e}\right)$; this gives a group of homomorphisms $g^{*}: K_{H}\left(\mathcal{B}_{e}\right) \rightarrow K_{H}\left(\mathcal{B}_{e}\right)$. Similarly we get a group of homomorphisms $g^{*}: K_{H}\left(\Lambda_{e}\right) \rightarrow K_{H}\left(\Lambda_{e}\right)$. These homomorphisms commute with the actions of $\tilde{T}_{w_{0}}$, with the Serre-Grothendieck duality and with $\Upsilon$. Also they are $\mathcal{A}$-linear. Hence they commute with $\tilde{\beta}, \beta$ in 5.5 . Also, the inner product $(\|)$ is preserved by $g^{*}$ up to the automorphism of $R_{H}$ induced by $g: H \rightarrow H$. It follows that $g^{*}$ maps $\mathbf{B}_{\mathcal{B}_{e}}^{ \pm}$into itself and $\mathbf{B}_{\Lambda_{e}}^{ \pm}$into itself.

5.19. The results of [L6] suggest that the elements of $\mathbf{B}_{\Lambda_{e}}^{ \pm}$are, up to sign, vector bundles on $\Lambda_{e}$.

5.20. The fact that a nilpotent element of type $E_{8}\left(b_{6}\right)$ has an exceptional behaviour is not entirely surprising. Consider a simple algebraic group $G^{\prime}$ over $\bar{F}_{p}$ with a fixed split $F_{p}$-rational structure and let $u$ be a unipotent element in $G^{\prime}\left(F_{p}\right)$. Assume that $p$ is a good prime for $G^{\prime}$. Consider the action of the Frobenius map on the set of irreducible components of the variety of Borel subgroups containing $u$. This action is non-trivial if $G^{\prime}$ is of type $E_{8}, u$ is of type $E_{8}\left(b_{6}\right)$ and $p=-1$ $\bmod 3[\mathrm{BS}]$; it is trivial in all other cases. 
5.21. We want to give a heuristic motivation for our choice of $\Upsilon$ in 5.7. Assume that 5.16(a) holds. Then the group homomorphism $\left.\tilde{\beta}\right|_{v=1}: K\left(\mathcal{B}_{e}\right) \rightarrow K\left(\mathcal{B}_{e}\right)$ keeps fixed each element of a $\mathbf{Z}$-basis of $K\left(\mathcal{B}_{e}\right)$ hence is the identity map. In other words,

$$
\left.\Upsilon\right|_{v=1}=\left.\left.(-1)^{\nu} D_{\mathcal{B}_{e}}\right|_{v=1} \tilde{T}_{w_{0}}\right|_{v=1}: K\left(\mathcal{B}_{e}\right) \rightarrow K\left(\mathcal{B}_{e}\right) .
$$

Now $H_{2 b(e)}\left(\mathcal{B}_{e}, \mathbf{Z}\right)$ is naturally a quotient of $K\left(\mathcal{B}_{e}\right)$; on this quotient $\left.D_{\mathcal{B}_{e}}\right|_{v=1}$ becomes $(-1)^{b(e)}$ and $\left.\tilde{T}_{w_{0}}\right|_{v=1}$ becomes $(-1)^{\nu}$ times the action of $w_{0}$ in the Springer representation. Hence on this quotient we should have

(a) $\left.\Upsilon\right|_{v=1}=(-1)^{b(e)} w_{0}$.

This justifies our choice of $\Upsilon$ at least in the case where $(e, h, f)$ is distinguished. (The action of $\iota$ in 5.4 on $I_{e}$ is trivial since it comes from a $\mathbf{C}^{*}$-action on $\mathcal{B}_{e}$.)

We have not verified that our choice of $\Upsilon$ satisfies (a) in the non-distinguished case.

\section{The VARIETIES $\mathfrak{Z}, \tilde{\mathfrak{Z}}$}

6.1. In this section we assume that we are given a parabolic subalgebra $\mathfrak{p}$ of $\mathfrak{g}$ with Levi subalgebra $\mathfrak{l}$ such that $e, h, f$ and the Lie algebra of $C$ are contained in $\mathfrak{l}$. Let $\mathfrak{n}=\mathfrak{n}_{\mathfrak{p}}$. Let $P, L, U$ be the closed subgroups of $G$ corresponding to $\mathfrak{p}, \mathfrak{l}, \mathfrak{n}$. Let

$$
\begin{gathered}
\Pi^{\prime}=e+\mathfrak{z}_{\mathfrak{p}}(f), \quad \Pi=\Pi^{\prime} \cap \mathfrak{g}_{n i l}, \quad \tilde{\Pi}=e+\mathfrak{z}_{\mathfrak{n}}(f), \\
\mathfrak{Z}=\{(y, \mathfrak{b}) \in \Pi \times \mathcal{B} \mid y \in \mathfrak{b}\}, \quad \tilde{\mathfrak{Z}}=\{(y, \mathfrak{b}) \in \tilde{\Pi} \times \mathcal{B} \mid y \in \mathfrak{b}\} .
\end{gathered}
$$

We have

$$
\mathcal{B}_{e} \subset \tilde{\mathfrak{Z}} \subset \mathfrak{Z} \subset \Lambda_{e}
$$

since $\{e\} \subset \tilde{\Pi} \subset \Pi \subset \Sigma_{e}$.

Let $K$ be the subset of $I$ such that $\mathfrak{p}$ is of type $K$. Let $W^{*}$ be the subgroup of $W$ generated by $\left\{\sigma_{i} \mid i \in K\right\}$. Let $W_{*}$ be the set of all $w \in W$ such that $w$ has minimal length in the coset $w W^{*}$. For any $w \in W_{*}$, let $\mathbf{o}(w)$ be the set of all $\mathfrak{b} \in \mathcal{B}$ such that for some $\mathfrak{b}^{\prime} \in \mathcal{B}, \mathfrak{b}^{\prime} \subset \mathfrak{p}$ the relative position of $\mathfrak{b}, \mathfrak{b}^{\prime}$ is $w$. Then $\mathbf{o}(w)$ is a $P$-orbit on $\mathcal{B}$ (for the conjugation action) and $w \mapsto \mathbf{o}(w)$ is a bijection between $W_{*}$ and the set of $P$-orbits on $\mathcal{B}$.

For $w \in W_{*}$ we define

$$
\mathfrak{Z}_{w}=\{(y, \mathfrak{b}) \in \Pi \times \mathbf{o}(w) \mid y \in \mathfrak{b}\}, \quad \tilde{\mathfrak{Z}}_{w}=\{(y, \mathfrak{b}) \in \tilde{\Pi} \times \mathbf{o}(w) \mid y \in \mathfrak{b}\},
$$

so that we have partitions

$$
\mathfrak{Z}=\bigcup_{w \in W_{*}} \mathfrak{Z}_{w}, \quad \tilde{\mathfrak{Z}}=\bigcup_{w \in W_{*}} \tilde{\mathfrak{Z}}_{w}
$$

into locally closed pieces.

Let $\underline{\mathcal{B}}_{e} \subset \underline{\Lambda}_{e}$ be the varieties defined like $\mathcal{B}_{e} \subset \Lambda_{e}$ in terms of $\mathfrak{p} / \mathfrak{n}$ and the images of $e, h, f$ in $\mathfrak{p} / \mathfrak{n}$ instead of $\mathfrak{g}$ and $e, h, f$. Thus,

$$
\begin{aligned}
& \underline{\mathcal{B}}_{e}=\{(e, \mathfrak{b}) \mid \mathfrak{b} \in \mathcal{B}, \mathfrak{b} \subset \mathfrak{p}, e \in \mathfrak{b}\}, \\
& \underline{\Lambda}_{e}=\left\{(z, \mathfrak{b}) \mid \mathfrak{b} \in \mathcal{B}, \mathfrak{b} \subset \mathfrak{p}, z \in(\Pi+\mathfrak{n}) / \mathfrak{n}, z \in \mathfrak{n}_{\mathfrak{b}} / \mathfrak{n}\right\} .
\end{aligned}
$$

(We identify in an obvious way the variety of Borel subalgebras of $\mathfrak{p} / \mathfrak{n}$ with the variety of Borel subalgebras of $\mathfrak{p}$.) 
For any Borel subgroup $B$ of $G$ we set $B^{!}=(B \cap P) U$ (a Borel subgroup of $P$ ). Similarly, for any Borel subalgebra $\mathfrak{b}$ of $\mathfrak{g}$ we set $\mathfrak{b}^{!}=(\mathfrak{b} \cap \mathfrak{p})+\mathfrak{n}$ (a Borel subalgebra of $\mathfrak{p})$.

6.2. For any $w \in W_{*}$ we define morphisms $\tilde{\gamma}: \tilde{\mathfrak{Z}}_{w} \rightarrow \underline{\mathcal{B}}_{e}$ by $(y, \mathfrak{b}) \mapsto\left(e, \mathfrak{b}^{!}\right)$and $\gamma: \mathfrak{Z}_{w} \rightarrow \underline{\Lambda}_{e}$ by $(y, \mathfrak{b}) \mapsto\left(y+\mathfrak{n}, \mathfrak{b}^{!}\right)$.

Note that the $H$-action on $\Lambda_{e}$ leaves $\mathfrak{Z}, \mathfrak{Z}_{w}, \tilde{\mathfrak{Z}}, \tilde{\mathfrak{Z}}_{w}$ stable. $H$ also acts naturally on $\underline{\mathcal{B}}_{e}, \underline{\Lambda}_{e}$ in such a way that $\tilde{\gamma}, \gamma$ are $H$-equivariant.

Theorem 6.3. (a) $\mathfrak{Z}_{w}$ is a smooth variety of pure dimension $\operatorname{dim} \mathfrak{z}_{\mathfrak{p}}(f)-\operatorname{dim} \mathfrak{t}$.

(b) $\gamma$ can be regarded as an $H$-equivariant vector bundle of dimension $\operatorname{dim} \mathfrak{z}_{\mathfrak{n}}(f)$.

(c) $\tilde{\gamma}$ can be regarded as an $H$-equivariant vector bundle of dimension $\operatorname{dim} \mathfrak{z} \mathfrak{n}(f)$.

The proof of (a),(b),(c) will be given in $6.11,6.12,6.13$ respectively.

Lemma 6.4. Let $\mathfrak{b}_{1}, \mathfrak{b}_{2} \in \mathbf{o}(w)$. The following conditions are equivalent:

(a) $\mathfrak{b}_{1}^{!}=\mathfrak{b}_{2}^{!}$;

(b) $\operatorname{Ad}(u) \mathfrak{b}_{1}=\mathfrak{b}_{2}$ for some $u \in U$.

Assume that (a) holds. Let $B_{1}$ be the Borel subgroup with Lie algebra $\mathfrak{b}_{1}$. We have $\operatorname{Ad}(p) \mathfrak{b}_{1}=\mathfrak{b}_{2}$ for some $p \in P$. Hence $\mathfrak{b}_{1}^{!}=\mathfrak{b}_{2}^{!}=\left(\operatorname{Ad}(p) \mathfrak{b}_{1}\right)^{!}=\operatorname{Ad}(p)\left(\mathfrak{b}_{1}^{!}\right)$. Thus $p$ normalizes $\mathfrak{b}_{1}^{!}$so that $p \in B_{1}^{!}$and $p=u_{1} p_{1}$ with $u_{1} \in U, p_{1} \in B_{1} \cap P$. Then $\mathfrak{b}_{2}=\operatorname{Ad}(p) \mathfrak{b}_{1}=\operatorname{Ad}\left(u_{1}\right) \operatorname{Ad}\left(p_{1}\right) \mathfrak{b}_{1}=\operatorname{Ad}\left(u_{1}\right) \mathfrak{b}_{1}$ and $(\mathrm{b})$ holds.

Assume that (b) holds. We have $\mathfrak{b}_{2}^{!}=\left(\operatorname{Ad}(u) \mathfrak{b}_{1}\right)^{!}=\operatorname{Ad}(u)\left(\mathfrak{b}_{1}\right)^{!}=\mathfrak{b}_{1}^{!}$and (a) holds. The lemma is proved.

6.5. We set

$$
R=\{(y, \mathfrak{b}) \mid \mathfrak{b} \in \mathbf{o}(w), y \in \mathfrak{p} \cap \mathfrak{b}\}, \quad \underline{R}=\{(z, \mathfrak{b}) \mid \mathfrak{b} \in \mathcal{B}, \mathfrak{b} \subset \mathfrak{p}, z \in \mathfrak{b} / \mathfrak{n}\} .
$$

Note that $R$ is a smooth variety: the map $p r_{2}: R \rightarrow \mathbf{o}(w)$ makes $R$ into a vector bundle over $\mathbf{o}(w)$. Also $\underline{R}$ is a smooth variety: a vector bundle over $\{\mathfrak{b} \in \mathcal{B} \mid \mathfrak{b} \subset \mathfrak{p}\}$. Let $\pi: R \rightarrow \underline{R}$ be the morphism defined by $\pi(y, \mathfrak{b})=\left(y+\mathfrak{n}, \mathfrak{b}^{!}\right)$.

Let $S$ be the connected centre of $L$. We have $S \subset C$. Let $\mathbf{o}(w)^{S}$ be the fixed point set of the conjugation action of $S$ on $\mathbf{o}(w)$. The map $\mathbf{o}(w)^{S} \rightarrow\{\mathfrak{b} \in \mathcal{B} \mid \mathfrak{b} \subset \mathfrak{p}\}$ given by $\mathfrak{b} \mapsto \mathfrak{b}$ ! is an isomorphism.

The fixed point set of the natural $S$-action on $R$ is

$$
R_{0}=\left\{\left(y_{0}, \mathfrak{b}_{0}\right) \mid \mathfrak{b}_{0} \in \mathbf{o}(w)^{S}, y_{0} \in \mathfrak{l} \cap \mathfrak{b}_{0}\right\} .
$$

We have $R_{0} \subset R$ and the restriction of $\pi$ defines an isomorphism $R_{0} \stackrel{\sim}{\rightarrow} \underline{R}$.

Let $\left(y_{0}, \mathfrak{b}_{0}\right) \in R_{0}$. Let $F$ be the fibre of $\pi$ at $\pi\left(y_{0}, \mathfrak{b}_{0}\right)$. Consider the map $U \times\left(\mathfrak{n} \cap \mathfrak{b}_{0}\right) \rightarrow F$ given by

$$
(u, x) \mapsto\left(\operatorname{Ad}(u)\left(y_{0}+x\right), \operatorname{Ad}(u) \mathfrak{b}_{0}\right) .
$$

This map is constant on the orbits of the free action of $U \cap B_{0}$ on $U \times\left(\mathfrak{n} \cap \mathfrak{b}_{0}\right)$ given by

$$
u_{1}:(u, x) \mapsto\left(u u_{1}^{-1}, \operatorname{Ad}\left(u_{1}\right)\left(y_{0}+x\right)-y_{0}\right)
$$

hence it induces a morphism

$$
\frac{U \times\left(\mathfrak{n} \cap \mathfrak{b}_{0}\right)}{U \cap B_{0}} \rightarrow F
$$

Lemma 6.6. This is an isomorphism. In particular, $F$ is an irreducible variety of dimension $\operatorname{dim} U=\operatorname{dim} \mathfrak{n}$. 
We only prove bijectivity. The injectivity is obvious. The surjectivity follows from Lemma 6.4. The lemma is proved.

6.7. We can choose a homomorphism of algebraic groups $\tau^{\prime}: \mathbf{C}^{*} \rightarrow S$ such that $\lambda \mapsto \operatorname{Ad}\left(\tau^{\prime}(\lambda)\right)$ has $>0$ weights on $\mathfrak{n}$. We then define a $\mathbf{C}^{*}$-action on $R$ by

$$
\lambda:(y, \mathfrak{b}) \mapsto\left(\operatorname{Ad}\left(\tau^{\prime}(\lambda)\right) y, \operatorname{Ad}\left(\tau^{\prime}(\lambda)\right) \mathfrak{b}\right)
$$

and a $\mathbf{C}^{*}$-action on $\underline{R}$ by the identity map. Note that $\pi: R \rightarrow \underline{R}$ is $\mathbf{C}^{*}$-equivariant.

We show that, for any $(y, b) \in F$ we have $\lim _{\lambda \rightarrow 0} \lambda(y, \mathfrak{b})=\left(y_{0}, \mathfrak{b}_{0}\right)$.

We may write $(y, \mathfrak{b})=\left(\operatorname{Ad}(u)\left(y_{0}+x\right), \operatorname{Ad}(u) \mathfrak{b}_{0}\right)$ for some $(u, x) \in U \times\left(\mathfrak{n} \cap \mathfrak{b}_{0}\right)$. We have

$$
\begin{aligned}
& \lambda(y, \mathfrak{b})=\left(\operatorname{Ad}\left(\tau^{\prime}(\lambda)\right) \operatorname{Ad}(u)\left(y_{0}+x\right), \operatorname{Ad}\left(\tau^{\prime}(\lambda)\right) \operatorname{Ad}(u) \mathfrak{b}_{0}\right) \\
& =\left(\operatorname{Ad}\left(\tau^{\prime}(\lambda) u \tau^{\prime}\left(\lambda^{-1}\right)\right)\left(y_{0}+\operatorname{Ad}\left(\tau^{\prime}(\lambda)\right) x\right), \operatorname{Ad}\left(\tau^{\prime}(\lambda) u \tau^{\prime}\left(\lambda^{-1}\right)\right) \mathfrak{b}_{0}\right) .
\end{aligned}
$$

Now $\lim _{\lambda \rightarrow 0} \tau^{\prime}(\lambda) u \tau^{\prime}\left(\lambda^{-1}\right)=1$ and $\lim _{\lambda \rightarrow 0} \operatorname{Ad}\left(\tau^{\prime}(\lambda)\right) x=0$ (by the choice of $\tau^{\prime}$ ); our assertion follows.

6.8. Let $\tilde{R}$ be a smooth, connected, $\mathbf{C}^{*}$-equivariant compactification of $R$. Let $\mu$ be the connected component of the fixed point set of $\mathbf{C}^{*}$ that contains $R_{0}$ (which is clearly connected). Let $\mu^{+}$be the set of points $\xi \in \tilde{R}$ which flow as $\lambda \rightarrow 0$ to a point of $\mu$ (denoted $r(\xi)$ ). By [BB], $r: \mu^{+} \rightarrow \mu$ may be regarded as a vector bundle. By the results in 6.7, we have $R \subset \mu^{+}$. More precisely, we have $R \subset r^{-1}\left(R_{0}\right) \subset \mu^{+}$.

We show that $R=r^{-1}\left(R_{0}\right)$. It is enough to show that $r^{-1}\left(y_{0}, \mathfrak{b}_{0}\right) \subset R$ for any $\left(y_{0}, \mathfrak{b}_{0}\right) \in R_{0}$. We may assume that $\left(y_{0}, \mathfrak{b}_{0}\right)$ is the point in 6.5 . Since $R$ is open in $\tilde{R}$, we have $\operatorname{dim} \mu^{+}=\operatorname{dim} R$. Hence

$$
\operatorname{dim} r^{-1}\left(y_{0}, \mathfrak{b}_{0}\right)=\operatorname{dim} \mu^{+}-\operatorname{dim} \mu=\operatorname{dim} R-\operatorname{dim} \mu \leq \operatorname{dim} R-\operatorname{dim} R_{0}=\operatorname{dim} F .
$$

But $F \subset r^{-1}\left(y_{0}, \mathfrak{b}_{0}\right)$, hence $\operatorname{dim} r^{-1}\left(y_{0}, \mathfrak{b}_{0}\right)=\operatorname{dim} F$. Thus, $F$ must contain some open subset of $r^{-1}\left(y_{0}, \mathfrak{b}_{0}\right)$. Since the $\mathbf{C}^{*}$-action acts as contraction of $r^{-1}\left(y_{0}, \mathfrak{b}_{0}\right)$ to $\left(y_{0}, \mathfrak{b}_{0}\right)$ and $F$ is $\mathbf{C}^{*}$-stable, it follows that $F=r^{-1}\left(y_{0}, \mathfrak{b}_{0}\right)$. Thus, $R=r^{-1}\left(R_{0}\right)$ as claimed. We see that

(a) $\pi: R \rightarrow R_{0}=\underline{R}$ is naturally a vector bundle with fibres of dimension $\operatorname{dim} \mathfrak{n}$.

Lemma 6.9. (a) $\Pi^{\prime}$ is a transversal slice to the $P$-orbit of $e$ in $\mathfrak{p}$.

(b) The map $P \times \Pi^{\prime} \mapsto \mathfrak{p}$ (adjoint action of $P$ on $\mathfrak{p}$ ) is smooth with fibres of pure dimension $\operatorname{dim} \Pi^{\prime}=\operatorname{dim} \mathfrak{z}_{\mathfrak{p}}(f)$.

(a) is a generalization of a result of Slodowy $[\mathrm{S}]$ (which is the case $\mathfrak{p}=\mathfrak{l}=\mathfrak{g}$.) The proof is similar to that in $[\mathrm{S}]$. The infinitesimal version of the lemma is that $\mathfrak{p}=[\mathfrak{p}, e] \oplus \mathfrak{z}_{\mathfrak{p}}(f)$ which follows from the representation theory of $\mathfrak{s l}_{2}$ applied to the $\mathfrak{s l}_{2}$-module $\mathfrak{p}$. It follows that $\Pi^{\prime}$ is a transversal slice to the $P$-orbit of $e$ in $\mathfrak{p}$ at least near $e$. But we can remove the words "near $e$ " using the action of $\mathbf{C}^{*}$ on $\Pi^{\prime}$ (see 1.8 ) which has only $<0$ weights on $\mathfrak{z}_{\mathfrak{p}}(f)$. This proves (a). Now (b) is just another way to state (a).

6.10. Let $\dot{\Pi}^{\prime}=\left\{(y, \mathfrak{b}) \in \Pi^{\prime} \times \mathbf{o}(w) \mid y \in \mathfrak{b}\right\}$. We have a cartesian diagram

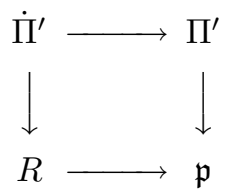


where the vertical maps are inclusions and the horizontal maps are given by the first projection. Hence we have a cartesian diagram

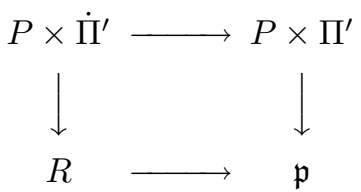

where the vertical maps are given by the Ad-action. Using this and 6.9(b), we deduce that

(a) $P \times \dot{\Pi}^{\prime} \rightarrow R$ (adjoint action) is smooth with fibres of pure dimension $\operatorname{dim} \Pi^{\prime}=$ $\operatorname{dim} \mathfrak{z}_{\mathfrak{p}}(f)$.

6.11. Let $\mathfrak{t}$ be the canonical Cartan algebra of $\mathfrak{p} / \mathfrak{n}$. We have a canonical map $\delta: \underline{R} \rightarrow \underline{\mathfrak{t}}$ which associates to $(z, \mathfrak{b}) \in \underline{R}$ the image of $z$ in $\mathfrak{b} / \mathfrak{n}_{\mathfrak{b}}=\underline{\mathfrak{t}}$. By a well known property of the reductive Lie algebra $\mathfrak{p} / \mathfrak{n}, \delta$ is smooth with fibres of pure $\operatorname{dimension} \operatorname{dim} \mathfrak{p} / \mathfrak{n}-\operatorname{dim} \underline{t}$. Combining this with $6.10(\mathrm{a}), 6.8(\mathrm{a})$, we see that the composition

$$
P \times \dot{\Pi}^{\prime} \rightarrow R \stackrel{\pi}{\rightarrow} \underline{R} \stackrel{\delta}{\rightarrow} \underline{\mathfrak{t}}
$$

is smooth with fibres of pure $\operatorname{dimension} \operatorname{dim} \mathfrak{z} \mathfrak{p}(f)+\operatorname{dim} \mathfrak{n}+\operatorname{dim} \mathfrak{p} / \mathfrak{n}-\operatorname{dim} \underline{\mathfrak{t}}$. But this map factorizes as

$$
P \times \dot{\Pi}^{\prime} \stackrel{p r_{2}}{\longrightarrow} \dot{\Pi}^{\prime} \stackrel{\rho}{\longrightarrow} \underline{\mathfrak{t}}
$$

where $\rho$ is the restriction of $\delta \pi$. (We use that $\delta \pi$ is constant on $P$-orbits.) Hence the composition $P \times \dot{\Pi}^{\prime} \stackrel{p r_{2}}{\longrightarrow} \dot{\Pi}^{\prime} \stackrel{\rho}{\longrightarrow} \underline{\mathfrak{t}}$ is smooth with fibres of pure dimension $\operatorname{dim} \mathfrak{z}_{\mathfrak{p}}(f)+\operatorname{dim} \mathfrak{p}-\operatorname{dim} \underline{\mathfrak{t}}$. It follows that $\dot{\Pi}^{\prime} \stackrel{\rho}{\rightarrow} \underline{\mathfrak{t}}$ is smooth with fibres of pure dimension $\operatorname{dim} \mathfrak{z}_{\mathfrak{p}}(f)-\operatorname{dim} \underline{\mathfrak{t}}$. In particular, its fibre at 0 , that is $\mathfrak{Z}_{w}$, is smooth of pure dimension $\operatorname{dim} \mathfrak{z}_{\mathfrak{p}}(f)-\operatorname{dim} \underline{\mathfrak{t}}$. Thus, 6.3(a) is proved.

6.12. Let $\mathfrak{Z}_{w}^{S}$ be the fixed point set of the $S$-action on $\mathfrak{Z}_{w}$ (restriction of the $S$ action on $R$ ). We have

$$
\mathfrak{Z}_{w}^{S}=\left\{\left(y_{0}, \mathfrak{b}_{0}\right) \mid \mathfrak{b}_{0} \in \mathbf{o}(w)^{S}, y_{0} \in e+\mathfrak{z} \mathfrak{r}(f), y_{0} \in \mathfrak{n}_{\mathfrak{b}_{0}}\right\} .
$$

Hence $\pi: R \rightarrow \underline{R}$ restricts to an isomorphism $\mathfrak{Z}_{w}^{S} \stackrel{\sim}{\rightarrow} \underline{\Lambda}_{e}$.

Since $\pi: R \rightarrow \underline{R}$ may be regarded as a vector bundle (by 6.8(a)) with a linear fibre preserving $\mathbf{C}^{*}$-action with $>0$ weights (see 6.7$)$ and $\mathfrak{Z}_{w}$ is a closed, smooth $\mathbf{C}^{*}$-stable subvariety of $R$, we see that $\lim _{\lambda \rightarrow 0} \lambda(y, \mathfrak{b})$ exists for any $(y, \mathfrak{b})$ and is exactly the point of $\mathfrak{Z}_{w}^{\mathbf{C}^{*}}$ corresponding to $\pi(y, \mathfrak{b}) \in \underline{\Lambda}_{e}$. (The $\mathbf{C}^{*}$-action considered here is the one in 6.7.)

We choose an $H$-equivariant smooth compactification of $\mathfrak{Z}_{w}$; this is also $\mathbf{C}^{*}$ equivariant since the action of $\mathbf{C}^{*}$ in 6.7 is part of the $H$-action. Arguing as in 6.8 , we see that the canonical map $\mathfrak{Z}_{w} \rightarrow \mathfrak{Z}_{w}^{\mathrm{C}^{*}}=\mathfrak{Z}_{w}^{S}=\underline{\Lambda}_{e}$ is naturally an (Hequivariant) vector bundle with fibres of dimension

$$
\operatorname{dim} \mathfrak{Z}_{w}-\operatorname{dim} \underline{\Lambda}_{e}=\operatorname{dim} \mathfrak{z}_{\mathfrak{p}}(f)-\operatorname{dim} \mathfrak{t}-\left(\operatorname{dim} \mathfrak{z}_{\mathfrak{l}}(f)-\operatorname{dim} \mathfrak{t}\right)=\operatorname{dim} \mathfrak{z}_{\mathfrak{n}}(f) .
$$

This proves $6.3(\mathrm{~b})$. 
6.13. We consider the commutative diagram

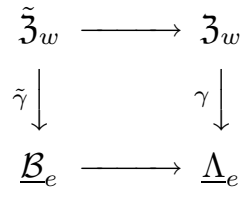

where the horizontal maps are the obvious inclusions. It is easy to see that this diagram is cartesian. Hence 6.3(c) follows from 6.3(b). Theorem 6.3 is proved.

6.14. For any subset $\mathcal{K}$ of $W_{*}$ we set $\mathfrak{Z}_{\mathcal{K}}=\bigcup_{w \in \mathcal{K}} \mathfrak{Z}_{w}, \tilde{\mathfrak{Z}}_{\mathcal{K}}=\bigcup_{w \in \mathcal{K}} \tilde{Z}_{w}$.

Let $\leq$ be the partial order on $W_{*}$ given by $w \leq w^{\prime}$ whenever $\mathbf{o}(w)$ is contained in the closure of $\mathbf{o}\left(w^{\prime}\right)$ in $\mathcal{B}$.

Let $w \in W_{*}$. We shall write $\mathfrak{Z}_{\leq w}, \tilde{\mathfrak{Z}}_{\leq w}$ instead of $\mathfrak{Z}_{\mathcal{K}}, \tilde{\mathfrak{Z}}_{\mathcal{K}}$, where $\mathcal{K}=\left\{w^{\prime} \mid w^{\prime} \leq\right.$ $w\}$; these are closed subvarieties of $\mathfrak{Z}, \tilde{\mathfrak{Z}}$. We shall write $\mathfrak{Z}_{<w}, \tilde{\mathfrak{Z}}_{<w}$ instead of $\mathfrak{Z}_{\mathcal{K}}, \tilde{\mathfrak{Z}}_{\mathcal{K}}$, where $\mathcal{K}=\left\{w^{\prime} \mid w^{\prime}<w\right\}$; these are closed subvarieties of $\mathfrak{Z}, \tilde{\mathfrak{Z}}$. We shall write $\mathfrak{Z}_{\geq w}, \tilde{\mathfrak{Z}}_{\geq w}$ instead of $\mathfrak{Z}_{\mathcal{K}}, \tilde{\mathfrak{Z}}_{\mathcal{K}}$ where $\mathcal{K}=\left\{w^{\prime} \mid w^{\prime} \geq w\right\}$; these are open subvarieties of $\mathfrak{Z}, \tilde{\mathfrak{Z}}$.

A subset $\mathcal{K}$ of $W_{*}$ is said to be closed if

$$
w \in \mathcal{K}, w^{\prime} \leq w \Longrightarrow w^{\prime} \in \mathcal{K} .
$$

In this case, $\mathfrak{Z}_{\mathcal{K}}, \tilde{\mathfrak{Z}}_{\mathcal{K}}$ are closed in $\mathfrak{Z}, \tilde{\mathfrak{Z}}$ respectively.

Lemma 6.15. Assume that $\mathcal{K} \subset W_{*}$ is closed.

(a) $K_{H}\left(\mathfrak{Z}_{\mathcal{K}}\right)$ is a free $R_{H}$-module of rank $|\mathcal{K}| \chi_{\underline{\mathcal{B}}_{e}}$.

(b) $K_{H}\left(\tilde{\mathfrak{Z}}_{\mathcal{K}}\right)$ is a free $R_{H}$-module of rank $|\mathcal{K}| \chi_{\underline{\mathcal{B}}_{e}}$.

We prove (a) by induction on $|\mathcal{K}|$. (Compare with the proof of 1.6.) If $\mathcal{K}=\emptyset$, the result is trivial. Assume now that $\mathcal{K} \neq \emptyset$. We choose $w \in \mathcal{K}$ which is maximal for $\leq$ on $\mathcal{K}$. Then $\mathcal{K}^{\prime}=\mathcal{K}-\{w\}$ is closed; we may assume that the result is true for $\mathcal{K}^{\prime}$. We have a partition $\mathfrak{Z}_{\mathcal{K}}=\mathfrak{Z}_{\mathcal{K}^{\prime}} \cup \mathfrak{Z}_{w}$ with $\mathfrak{Z}_{\mathcal{K}^{\prime}}$ closed in $\mathfrak{Z}_{\mathcal{K}}$ and $\mathfrak{Z}_{w}$ open in $\mathfrak{Z} \mathcal{K}$. Hence we have an associated long exact sequence in higher $K$-theory:

$$
K_{H}^{1}\left(\mathfrak{Z}_{w}\right) \stackrel{a^{\prime}}{\rightarrow} K_{H}^{0}\left(\mathfrak{Z}_{\mathcal{K}^{\prime}}\right) \rightarrow K_{H}^{0}\left(\mathfrak{Z}_{\mathcal{K}}\right) \rightarrow K_{H}^{0}\left(\mathfrak{Z}_{w}\right) \rightarrow 0 .
$$

If we take fixed point sets of the $S$-action, we get a partition $\mathfrak{Z}_{\mathcal{K}}^{S}=\mathfrak{Z}_{\mathcal{K}^{\prime}}^{S} \cup \mathfrak{Z}_{w}^{S}$ where, this time, $\mathfrak{Z}_{\mathcal{K}^{\prime}}^{S}$ and $\mathfrak{Z}_{w}^{S}$ are both open and closed in $\mathfrak{Z}_{\mathcal{K}}^{S}$. Hence in the corresponding long exact sequence in higher $K$-theory

$$
K_{H}^{1}\left(\mathfrak{Z}_{w}^{S}\right) \stackrel{a}{\rightarrow} K_{H}^{0}\left(\mathfrak{Z}_{\mathcal{K}^{\prime}}^{S}\right) \rightarrow K_{H}^{0}\left(\mathfrak{Z}_{\mathcal{K}}^{S}\right) \rightarrow K_{H}^{0}\left(\mathfrak{Z}_{w}^{S}\right) \rightarrow 0
$$

the boundary map $a$ is 0 . Let $x \in K^{1}\left(\mathfrak{Z}_{w}\right)$. We show that $a^{\prime}(x)=0$. Consider the commutative diagram

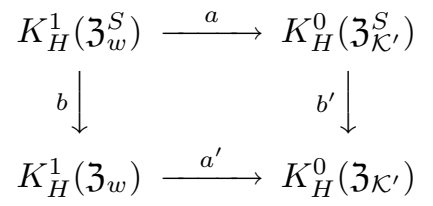

with vertical maps given by direct image induced by the inclusion. By $[\mathrm{Th}], b$ is an isomorphism after tensoring by $\mathfrak{R}$. Hence we can find $y \in K^{1}\left(\mathfrak{Z}_{w}^{S}\right)$ and $f \in R_{H}-\{0\}$ such that $f x=b(y)$. Then $f a^{\prime}(x)=a^{\prime} b(y)=b^{\prime} a(y)=0$ since $a=0$. By the induction hypothesis, $K_{H}^{0}\left(\mathfrak{Z}_{\mathcal{K}^{\prime}}\right)$ is free over $R_{H}$ hence from $f a^{\prime}(x)=0$ we 
deduce $a^{\prime}(x)=0$. Thus, we have $a^{\prime}=0$. Thus, we have an exact sequence of $R_{H}$-modules:

$$
0 \rightarrow K_{H}^{0}\left(\mathfrak{Z}_{\mathcal{K}^{\prime}}\right) \rightarrow K_{H}^{0}\left(\mathfrak{Z}_{\mathcal{K}}\right) \rightarrow K_{H}^{0}\left(\mathfrak{Z}_{w}\right) \rightarrow 0
$$

where $K_{H}^{0}\left(\mathfrak{Z}_{\mathcal{K}^{\prime}}\right)$ is free of rank $\left|\mathcal{K}^{\prime}\right| \chi_{\underline{\mathcal{B}}_{e}}$ and $K_{H}^{0}\left(\mathfrak{Z}_{w}\right)$ is free of rank $\chi_{\underline{\mathcal{B}}_{e}}$ (see 6.3(b), $1.14(\mathrm{a}))$. It follows that $K_{H}^{0}\left(\mathfrak{Z}_{\mathcal{K}}\right)$ is free of rank $|\mathcal{K}| \chi_{\underline{\mathcal{B}}_{e}}$. This proves (a). The proof of (b) is entirely similar.

6.16. A similar argument shows that we have exact sequences of $R_{H}$-modules:

$$
\begin{aligned}
& 0 \rightarrow K_{H}\left(\mathfrak{Z}_{\mathcal{K}}\right) \rightarrow K_{H}(\mathfrak{Z}) \rightarrow K_{H}\left(\mathfrak{Z}-\mathfrak{Z}_{\mathcal{K}}\right) \rightarrow 0, \\
& 0 \rightarrow K_{H}\left(\tilde{\mathfrak{Z}}_{\mathcal{K}}\right) \rightarrow K_{H}(\tilde{\mathfrak{Z}}) \rightarrow K_{H}\left(\tilde{\mathfrak{Z}}-\tilde{\mathfrak{Z}}_{\mathcal{K}}\right) \rightarrow 0,
\end{aligned}
$$

which allows us to identify $K_{H}\left(\mathfrak{Z}_{\mathcal{K}}\right)$ with an $R_{H}$-submodule of $K_{H}(\mathfrak{Z})$ and $K_{H}\left(\tilde{\mathfrak{Z}}_{\mathcal{K}}\right)$ with an $R_{H}$-submodule of $K_{H}(\tilde{\mathfrak{Z}})$. Note that

(a) $K_{H}\left(\mathfrak{Z}_{\mathcal{K}}\right)=\sum_{w \in \mathcal{K}} K_{H}\left(\mathfrak{Z}_{\leq w}\right)$,

(b) $K_{H}\left(\tilde{\mathfrak{Z}}_{\mathcal{K}}\right)=\sum_{w \in \mathcal{K}} K_{H}\left(\tilde{\mathfrak{Z}}_{\leq w}\right)$,

as submodules of $K_{H}(\mathfrak{Z}), K_{H}(\tilde{\mathfrak{Z}})$ respectively.

We prove (a) by induction on $|\mathcal{K}|$. When $|\mathcal{K}|=0$ the result is obvious. Assume now that $|\mathcal{K}|>0$. We choose $w \in \mathcal{K}$ which is maximal for $\leq$ on $\mathcal{K}$. Then $\mathcal{K}^{\prime}=$ $\mathcal{K}-\{w\}$ is closed. Let $\xi \in K_{H}\left(\mathfrak{Z}_{\mathcal{K}}\right)$.

By the exact sequence 6.15 (c) for $\mathfrak{Z}_{\leq w}$ instead of $\mathfrak{Z}_{\mathcal{K}}$, we see that there exists $\xi^{\prime} \in K_{H}\left(\mathfrak{Z}_{\leq w}\right)$ such that $\xi, \xi^{\prime}$ have the same image in $K_{H}\left(\mathfrak{Z}_{w}\right)$. By the exactness of $6.15(\mathrm{c})$, the difference $\xi-\xi^{\prime}$ belongs to $K_{H}\left(\mathfrak{Z}_{\mathcal{K}^{\prime}}\right)$. By the induction hypothesis, $K_{H}\left(\mathfrak{Z} \mathcal{K}^{\prime}\right)$ is contained in the right hand side of (a). Hence $\xi$ is contained in the right hand side of (a). Thus, the left hand side of (a) is contained in the right hand side. The reverse inclusion is obvious. This proves (a). The proof of (b) is entirely similar.

Theorem 6.17. (a) $K_{H}(\mathfrak{Z})$ and $K_{H}(\tilde{\mathfrak{Z}})$ are free $R_{H}$-modules of the same rank as $K_{H}\left(\mathcal{B}_{e}\right)$ and $K_{H}\left(\Lambda_{e}\right)$.

(b) The direct image maps $K_{H}\left(\mathcal{B}_{e}\right) \rightarrow K_{H}(\tilde{\mathfrak{Z}}) \rightarrow K_{H}(\mathfrak{Z}) \rightarrow K_{H}\left(\Lambda_{e}\right)$ (induced by the inclusions $\mathcal{B}_{e} \subset \tilde{\mathfrak{Z}} \subset \mathfrak{Z} \subset \Lambda_{e}$ ) are injective.

The fact that $K_{H}(\mathfrak{Z})$ and $K_{H}(\tilde{\mathfrak{Z}})$ are free $R_{H}$-modules of finite rank is a special case of Lemma 6.15. This implies the remaining statements since the maps in (b) become isomorphisms after tensoring by $\mathfrak{R}$ over $R_{H}$ (by [Th], since the $H$-fixed points are the same on all four varieties).

\section{7. $K_{H}(\mathfrak{Z}), K_{H}(\tilde{\mathfrak{Z}})$ AS INDUCED $\mathcal{H}$-MOdules}

7.1. In this section we preserve the setup of 6.1.

Given $w \in W_{*}$ and $i \in I$, let ${ }_{i} \mathbf{o}(w)$ be the set of all $\mathfrak{b} \in \mathcal{B}$ such that $\left(\mathfrak{b}, \mathfrak{b}^{\prime}\right) \in \overline{\mathcal{O}}_{i}$ for some $\mathfrak{b}^{\prime} \in \mathbf{o}(w)$. There are three possibilities:

(a) $l\left(\sigma_{i} w\right)=l(w)+1$ and $\sigma_{i} w \notin W_{*}$. Then $\sigma_{i} w=w \sigma_{j}$ for some $j \in K$ and ${ }_{i} \mathbf{O}(w)=\mathbf{o}(w)$

(b) $l\left(\sigma_{i} w\right)=l(w)+1$ and $\sigma_{i} w \in W_{*}$. Then $w<\sigma_{i} w$ and ${ }_{i} \mathbf{o}(w)=\mathbf{o}(w) \cup \mathbf{o}\left(\sigma_{i} w\right)$;

(c) $l\left(\sigma_{i} w\right)=l(w)-1$. Then $\sigma_{i} w \in W_{*}, \sigma_{i} w<w$ and ${ }_{i} \mathbf{o}(w)=\mathbf{o}(w) \cup \mathbf{o}\left(\sigma_{i} w\right)$.

In 7.2-7.7 we assume that $w, i$ are as in (c).

Lemma 7.2. $\mathfrak{Z}_{\leq w}$ and $\tilde{\mathfrak{Z}}_{\leq w}$ are $i$-saturated. 
The proof is immediate.

7.3. From 7.2 we see that $r_{i}: K_{H}\left(\mathfrak{Z}_{\leq w}\right) \rightarrow K_{H}\left(\mathfrak{Z}_{\leq w}\right)$ and $r_{i}: K_{H}\left(\tilde{\mathfrak{Z}}_{\leq w}\right) \rightarrow$ $K_{H}\left(\tilde{\mathfrak{Z}}_{\leq w}\right)$ are defined. (See 4.3.) Let $f: K_{H}\left(\mathfrak{Z}_{\leq \sigma_{i} w}\right) \rightarrow K_{H}\left(\mathfrak{Z}_{w}\right)$ be the composition

$$
K_{H}\left(\mathfrak{Z}_{\leq \sigma_{i} w}\right) \rightarrow K_{H}\left(\mathfrak{Z}_{\leq w}\right) \stackrel{r_{i}}{\longrightarrow} K_{H}\left(\mathfrak{Z}_{\leq w}\right) \stackrel{k^{*}}{\longrightarrow} K_{H}\left(\mathfrak{Z}_{w}\right)
$$

where the first map is direct image under $\mathfrak{Z}_{\leq \sigma_{i} w} \subset \mathfrak{Z}_{\leq w}$ and $k: \mathfrak{Z}_{w} \rightarrow \mathfrak{Z}_{\leq w}$ is the inclusion. Let $\tilde{f}: K_{H}\left(\tilde{\mathfrak{Z}}_{\leq \sigma_{i} w}\right) \rightarrow K_{H}\left(\tilde{\mathfrak{Z}}_{\leq w}\right)$ be the composition

$$
K_{H}\left(\tilde{\mathfrak{Z}}_{\leq \sigma_{i} w}\right) \rightarrow K_{H}\left(\tilde{\mathfrak{Z}}_{\leq w}\right) \stackrel{r_{i}}{\rightarrow} K_{H}\left(\tilde{\mathfrak{Z}}_{\leq w}\right) \stackrel{\tilde{k}^{*}}{\rightarrow} K_{H}\left(\tilde{\mathfrak{Z}}_{w}\right)
$$

where the first map is direct image under $\tilde{\mathfrak{Z}}_{\leq \sigma_{i} w} \subset \tilde{\mathfrak{Z}}_{\leq w}$ and $\tilde{k}: \tilde{\mathfrak{Z}}_{w} \rightarrow \tilde{\mathfrak{Z}}_{\leq w}$ is the inclusion.

Lemma 7.4. (a) The composition $K_{H}\left(\mathfrak{Z}_{<\sigma_{i} w}\right) \rightarrow K_{H}\left(\mathfrak{Z}_{\leq \sigma_{i} w}\right) \stackrel{f}{\rightarrow} K_{H}\left(\mathfrak{Z}_{w}\right)$ is zero.

(b) The composition $K_{H}\left(\tilde{\mathfrak{Z}}_{<\sigma_{i} w}\right) \rightarrow K_{H}\left(\tilde{\mathfrak{Z}}_{\leq \sigma_{i} w}\right) \stackrel{\tilde{f}}{\rightarrow} K_{H}\left(\tilde{\mathfrak{Z}}_{w}\right)$ is zero.

The proof is entirely analogous to that of $[\mathrm{L} 5,8.7(\mathrm{a})]$.

7.5. Using Lemma 7.4, the exact sequence

$$
0 \rightarrow K_{H}\left(\mathfrak{Z}_{<\sigma_{i} w}\right) \rightarrow K_{H}\left(\mathfrak{Z}_{\leq \sigma_{i} w}\right) \rightarrow K_{H}\left(\mathfrak{Z}_{\sigma_{i} w}\right) \rightarrow 0
$$

(a special case of the exact sequence 6.15(c)) and the analogous exact sequence

$$
0 \rightarrow K_{H}\left(\tilde{\mathfrak{Z}}_{<\sigma_{i} w}\right) \rightarrow K_{H}\left(\tilde{\mathfrak{Z}}_{\leq \sigma_{i} w}\right) \rightarrow K_{H}\left(\tilde{\mathfrak{Z}}_{\sigma_{i} w}\right) \rightarrow 0
$$

we see that

(a) there is a unique $R_{H}$-linear map $f^{\prime}: K_{H}\left(\mathfrak{Z}_{\sigma_{i} w}\right) \rightarrow K_{H}\left(\mathfrak{Z}_{w}\right)$ such that the composition $K_{H}\left(\mathfrak{Z}_{\leq \sigma_{i} w}\right) \stackrel{k^{* *}}{\longrightarrow} K_{H}\left(\mathfrak{Z}_{\sigma_{i} w}\right) \stackrel{f^{\prime}}{\longrightarrow} K_{H}\left(\mathfrak{Z}_{w}\right)$ is equal to $f$;

(b) there is a unique $R_{H}$-linear map $\tilde{f}^{\prime}: K_{H}\left(\tilde{\mathfrak{Z}}_{\sigma_{i} w}\right) \rightarrow K_{H}\left(\tilde{\mathfrak{Z}}_{w}\right)$ such that the composition $K_{H}\left(\tilde{\mathfrak{Z}}_{\leq \sigma_{i} w}\right) \stackrel{k^{*}}{\longrightarrow} K_{H}\left(\tilde{\mathfrak{Z}}_{\sigma_{i} w}\right) \stackrel{\tilde{f}^{\prime}}{\longrightarrow} K_{H}\left(\tilde{\mathfrak{Z}}_{w}\right)$ is equal to $\tilde{f}$.

Here $k^{\prime}$ denotes the open imbedding $\mathfrak{Z}_{\sigma_{i} w} \rightarrow \mathfrak{Z}_{\leq \sigma_{i} w}$ or $\tilde{\mathfrak{Z}}_{\sigma_{i} w} \rightarrow \tilde{\mathfrak{Z}}_{\leq \sigma_{i} w}$.

Lemma 7.6. (a) We have a commutative diagram

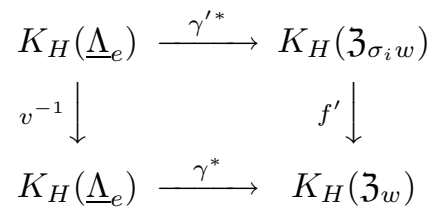

(b) We have a commutative diagram

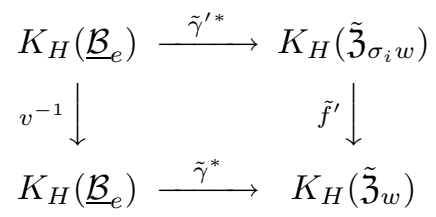

where $\gamma, \tilde{\gamma}$ are as in 6.2 and $\gamma^{\prime}, \tilde{\gamma}^{\prime}$ are the analogous maps for $\sigma_{i} w$ instead of $w$.

(c) $f^{\prime}, \tilde{f}^{\prime}$ are isomorphisms; $f, \tilde{f}$ are surjective. 
We prove (a). Since $\underline{\Lambda}_{e}$ is smooth, it is enough to show that $f^{\prime} \gamma^{\prime *} E=v^{-1} \gamma^{*} E \in$ $K_{H}\left(\mathfrak{Z}_{w}\right)$ for any $E \in \operatorname{Vec}_{H}\left(\underline{\Lambda}_{e}\right)$. Let $E_{1}=\gamma^{*} E \in \operatorname{Vec}_{H}\left(\mathfrak{Z}_{\sigma_{i} w}\right)$. Let $F^{\prime} \in$ $K_{H}\left(Z_{\leq \sigma_{i} w}\right)$ be such that $k^{\prime *} F_{1}=E_{1}$ in $K_{H}\left(Z_{\sigma_{i} w}\right)$. Let $F \in K_{H}\left(Z_{\leq w}\right)$ be the direct image of $F^{\prime}$ under $Z_{\leq \sigma_{i} w} \subset Z_{\leq w}$. Let

$$
\begin{aligned}
& V^{\prime}=\left\{\left(y, \mathfrak{b}, y, \mathfrak{b}^{\prime}\right) \in Z_{i} \mid\left(y, \mathfrak{b}^{\prime}\right) \in \mathfrak{Z}_{\sigma_{i} w}\right\}, \\
& \mathfrak{B}^{\prime}=\left\{\left(y, \mathfrak{b}, y, \mathfrak{b}^{\prime}\right) \in \bar{Z}_{i} \mid\left(y, \mathfrak{b}^{\prime}\right) \in \mathfrak{Z}_{\leq \sigma_{i} w}\right\}
\end{aligned}
$$

We have a cartesian diagram

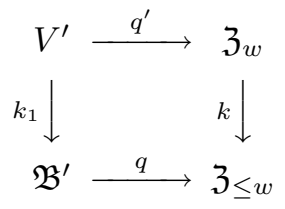

where $q^{\prime}\left(y, \mathfrak{b}, y, \mathfrak{b}^{\prime}\right)=(y, \mathfrak{b})$ (an isomorphism); $k_{1}$ is the obvious open imbedding; $q: \mathfrak{B}^{\prime} \rightarrow \mathfrak{Z}_{\leq w}$ is given by $q\left(y, \mathfrak{b}, y, \mathfrak{b}^{\prime}\right)=(y, \mathfrak{b})$. Then

$$
r_{i}(F)=q_{*}\left(v^{-1}\left(L^{\prime} \otimes L^{\prime \prime}\right) \otimes^{L}\left(\mathbf{C} \otimes F_{1}\right)\right)
$$

where $\otimes^{L}$ is computed in the smooth manifold $\Lambda^{2}$ relative to the closed subvarieties $\bar{Z}_{i}$ and $\Lambda \times \mathfrak{Z}_{\leq \sigma_{i} w}$ with intersection $\mathfrak{B}^{\prime}$. We have

$$
\begin{aligned}
& f\left(F_{1}\right)=k^{*} r_{i}(F)=k^{*} q_{*}\left(v^{-1}\left(L^{\prime} \otimes L^{\prime \prime}\right) \otimes_{\Lambda^{2}}^{L}\left(\mathbf{C} \otimes F_{1}\right)\right) \\
& =q_{*}^{\prime} k_{1}^{*}\left(v^{-1}\left(L^{\prime} \otimes L^{\prime \prime}\right) \otimes_{\Lambda^{2}}^{L}\left(\mathbf{C} \otimes F_{1}\right)\right) .
\end{aligned}
$$

Let $U^{\prime}$ be the set of all $\left(x, \mathfrak{b} ; x^{\prime}, \mathfrak{b}^{\prime}\right) \in \Lambda \times \Lambda$ such that $\mathfrak{b}^{\prime} \in \mathfrak{Z}_{\geq \sigma_{i} w}$ and such that the relative position of $\left(\mathfrak{b}, \mathfrak{b}^{\prime}\right)$ (an element of the Weyl group) contains the simple reflection $\sigma_{i}$ in any reduced expression. Then $U^{\prime}$ is open in $\Lambda \times \Lambda$ hence it is smooth. We have

$$
\begin{gathered}
U^{\prime} \cap \bar{Z}_{i}=U^{\prime} \cap Z_{i}=\left\{\left(x, \mathfrak{b} ; x, \mathfrak{b}^{\prime}\right) \in \Lambda^{2} \mid\left(\mathfrak{b}, \mathfrak{b}^{\prime}\right) \in \mathcal{O}_{i}, \mathfrak{b}^{\prime} \in \mathfrak{Z}_{\geq \sigma_{i} w}\right\} \\
U^{\prime} \cap\left(\Lambda \times \mathfrak{Z} \leq \sigma_{i} w\right)=U^{\prime} \cap\left(\Lambda \times \mathfrak{Z}_{\sigma_{i} w}\right) \\
U^{\prime} \cap \mathfrak{B}^{\prime}=V^{\prime}
\end{gathered}
$$

By [L5, 6.6], we have

$$
\begin{aligned}
& k_{1}^{*}\left(v^{-1}\left(L^{\prime} \otimes L^{\prime \prime}\right) \otimes_{\Lambda^{2}}^{L}\left(\mathbf{C} \otimes F_{1}\right)\right)=v^{-1}\left(L^{\prime} \otimes L^{\prime \prime}\right) \otimes_{U^{\prime}}^{L}\left(\mathbf{C} \otimes k^{\prime *} F_{1}\right) \\
& =v^{-1}\left(L^{\prime} \otimes L^{\prime \prime}\right) \otimes_{U^{\prime}}^{L}\left(\mathbf{C} \otimes E_{1}\right),
\end{aligned}
$$

where $\otimes_{U^{\prime}}^{L}$ is computed in the smooth manifold $U^{\prime}$ relative to the closed subvarieties $U^{\prime} \cap Z_{i}$ and $\Lambda \times \mathfrak{Z}_{\sigma_{i} w}$ with intersection $V^{\prime}=\mathfrak{Z}_{w}$. The subvarieties $U^{\prime} \cap Z_{i}$ and $\Lambda \times \mathfrak{Z}_{\sigma_{i} w}$ of $U^{\prime}$ are smooth (see 6.3(a)) and the intersection is transversal. (We have $\operatorname{dim}\left(U^{\prime} \cap Z_{i}\right)=\operatorname{dim} Z_{i}=2 \nu, \operatorname{dim}\left(\Lambda \times \mathfrak{Z}_{\sigma_{i} w}\right)=2 \nu+\operatorname{dim} \mathfrak{Z}_{\sigma_{i} w}=2 \nu+\operatorname{dim} \mathfrak{Z}_{w}$.) Hence $v^{-1}\left(L^{\prime} \otimes L^{\prime \prime}\right) \otimes_{U}^{L}\left(\mathbf{C} \otimes E_{1}\right)$ is the usual tensor product of the restrictions of the vector bundles $v^{-1}\left(L^{\prime} \otimes L^{\prime \prime}\right), \mathbf{C} \otimes E_{1}$ to $V^{\prime}=\mathfrak{Z}_{w}$. The restriction of $v^{-1}\left(L^{\prime} \otimes L^{\prime \prime}\right)$ is $v^{-1} \mathbf{C}$ (as in the proof of $[\mathrm{L} 5,8.8]$ ) and the restriction of $\mathbf{C} \otimes E_{1}$ is $\gamma^{*} E \in \operatorname{Vec}_{H}\left(\mathfrak{Z}_{w}\right)$. Thus (a) is proved. 
We prove (b). We claim that the diagram

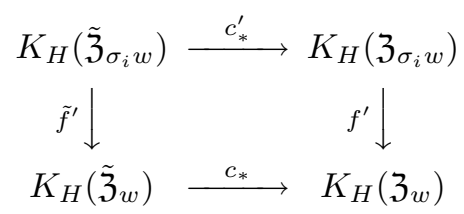

is commutative. Here $c, c^{\prime}$ are the obvious inclusions. It is enough to show that the analogous diagram

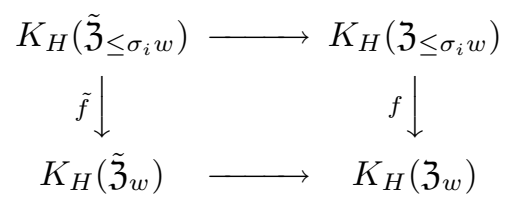

and this follows from the property 4.3(a) of the maps $r_{i}$. This establishes the claim. Using the claim and the injectivity of $c_{*}$, we see that in order to prove (b), it is enough to prove the commutativity of the diagram

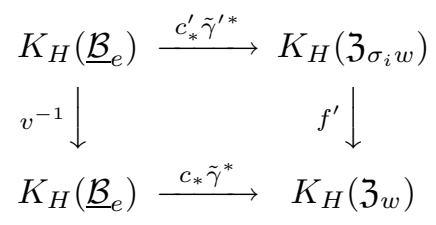

Using the cartesian diagram in 6.13 for $w$ and for $\sigma_{i} w$, we see that the commutativity of the last diagram is a consequence of (a). This proves (b).

The first assertion of (c) follows from (a),(b) using Theorem 6.3(b),(c). The second assertion of (c) follows from the first assertion of (c). The lemma is proved.

Lemma 7.7. (a) Let $\xi \in K_{H}\left(\mathfrak{Z}_{\leq w}\right)$. There exist $\xi^{\prime} \in K_{H}\left(\mathfrak{Z}_{\leq \sigma_{i} w}\right)$ and $\xi^{\prime \prime} \in$ $K_{H}\left(\mathfrak{Z}_{<w}\right)$ such that $\xi=r_{i}\left(j_{*}\left(\xi^{\prime}\right)\right)+j_{*}^{\prime}\left(\xi^{\prime \prime}\right)$. Here $j: \mathfrak{Z}_{\leq \sigma_{i} w} \rightarrow \mathfrak{Z}_{\leq w}, j^{\prime}: \mathfrak{Z}_{<w} \rightarrow \mathfrak{Z}_{\leq w}$ are the inclusions.

(b) Let $\tilde{\xi} \in K_{H}\left(\tilde{\mathfrak{Z}}_{\leq w}\right)$. There exist $\tilde{\xi}^{\prime} \in K_{H}\left(\tilde{\mathfrak{Z}}_{<\sigma_{i} w}\right)$ and $\tilde{\xi}^{\prime \prime} \in K_{H}\left(\tilde{\mathfrak{Z}}_{<w}\right)$ such that $\tilde{\xi}=r_{i}\left(\tilde{j}_{*}\left(\tilde{\xi}^{\prime}\right)\right)+\tilde{j}_{*}^{\prime}\left(\tilde{\xi}^{\prime \prime}\right)$. Here $\tilde{j}: \tilde{\mathfrak{Z}}_{\leq \sigma_{i} w} \rightarrow \tilde{\tilde{\mathfrak{Z}}}_{\leq w}, \tilde{j}^{\prime}: \tilde{\mathfrak{Z}}_{<w} \rightarrow \tilde{\mathfrak{Z}}_{\leq w}$ are the inclusions.

We prove (a). Consider the exact sequence

$$
0 \rightarrow K_{H}\left(\mathfrak{Z}_{<w}\right) \stackrel{j_{*}^{\prime}}{\longrightarrow} K_{H}\left(\mathfrak{Z}_{\leq w}\right) \stackrel{k^{*}}{\longrightarrow} K_{H}\left(\mathfrak{Z}_{w}\right) \rightarrow 0
$$

(a special case of the exact sequence 6.15(c)). By the surjectivity of $f$ in 7.6(c), there exists $\xi^{\prime} \in K_{H}\left(\mathfrak{Z}_{\leq \sigma_{i} w}\right)$ such that $k^{*} \xi=f\left(\xi^{\prime}\right)=k^{*} r_{i} j_{*}\left(\xi^{\prime}\right)$, hence $\xi-r_{i}\left(j_{*}\left(\xi^{\prime}\right)\right) \in$ Ker $k^{*}=\operatorname{Im} j_{*}^{\prime}$. Thus, $\xi-r_{i}\left(j_{*}\left(\xi^{\prime}\right)\right)=j_{*}^{\prime}\left(\xi^{\prime \prime}\right)$ for some $\xi^{\prime \prime} \in K_{H}\left(\mathfrak{Z}_{<w}\right)$. This proves (a). The proof of (b) is entirely similar.

Proposition 7.8. The imbeddings $K_{H}\left(\mathcal{B}_{e}\right) \rightarrow K_{H}(\tilde{\mathfrak{Z}}) \rightarrow K_{H}(\mathfrak{Z}) \rightarrow K_{H}\left(\Lambda_{e}\right)$ (see 6.17(b)) identify $K_{H}\left(\mathcal{B}_{e}\right), K_{H}(\tilde{\mathfrak{Z}}), K_{H}(\mathfrak{Z})$ with $\mathcal{H}$-submodules of $K_{H}\left(\Lambda_{e}\right)$.

This follows easily from 4.3(a). 
7.9. Note that $K, W^{*}$ play the same role for $P / U$ as $I, W$ for $G$.

By restricting line bundles in $\mathbf{X}$ to $\{\mathfrak{b} \in \mathcal{B} \mid \mathfrak{b} \subset \mathfrak{p}\}$ (identified naturally with the flag manifold of $\mathfrak{p} / \mathfrak{n}$ ) we obtain an isomorphism of $\mathbf{X}$ defined in terms of $G$ with $\mathbf{X}$ defined in terms of $P / U$. Hence $\mathcal{X}$ can be taken to be the same for $G$ and for $P / U$. We see that the affine Hecke algebra $\underline{\mathcal{H}}$ associated to $P / U$ may be naturally identified with the $\mathcal{A}$-subalgebra of $\mathcal{H}$ generated by the elements $\tilde{T}_{\sigma_{i}}, i \in K$ and $\theta_{x}, x \in \mathcal{X}$

Applying 4.4 to $P / U$ instead of $G$ we obtain natural $\underline{\mathcal{H}}$-module structures on $K_{H}\left(\underline{\mathcal{B}}_{e}\right), K_{H}\left(\underline{\Lambda}_{e}\right)$.

7.10. It is clear that $\mathfrak{Z}_{1}=\{(y, \mathfrak{b}) \in \mathfrak{Z} \mid \mathfrak{b} \subset \mathfrak{p}\}$ (resp. $\tilde{\mathfrak{Z}}_{1}=\{(y, \mathfrak{b}) \in \tilde{\mathfrak{Z}} \mid \mathfrak{b} \subset \mathfrak{p}\}$ is an $i$-saturated closed subvariety of $\mathfrak{Z}$ (resp. $\tilde{\mathfrak{Z}}$ ) for any $i \in K$. It follows that the images of the natural imbeddings $K_{H}\left(\mathfrak{Z}_{1}\right) \rightarrow K_{H}(\mathfrak{Z}), K_{H}\left(\tilde{\mathfrak{Z}}_{1}\right) \rightarrow K_{H}(\tilde{\mathfrak{Z}})$ are $\underline{\mathcal{H}}$ submodules of the $\mathcal{H}$-modules $K_{H}(\mathfrak{Z}), K_{H}(\tilde{\mathfrak{Z}})$. By $6.3, \mathfrak{Z}_{1}$ and $\tilde{\mathfrak{Z}}_{1}$ can be regarded as vector bundles (of the same dimension) over $\underline{\Lambda}_{e}, \underline{\mathcal{B}}_{e}$. Hence by taking inverse images we have isomorphisms

(a) $K_{H}\left(\underline{\Lambda}_{e}\right) \stackrel{\sim}{\rightarrow} K_{H}\left(\mathfrak{Z}_{1}\right), K_{H}\left(\underline{\mathcal{B}}_{e}\right) \stackrel{\sim}{\rightarrow} K_{H}\left(\tilde{\mathfrak{Z}}_{1}\right)$.

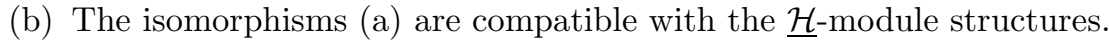

(We omit the proof.) We shall use (a) to identify $K_{H}\left(\underline{\mathcal{B}}_{e}\right)$ with the $\underline{\mathcal{H}}$-submodule $K_{H}\left(\tilde{\mathfrak{Z}}_{1}\right)$ of $K_{H}(\tilde{\mathfrak{Z}})$ and to identify $K_{H}\left(\underline{\Lambda}_{e}\right)$ with the $\underline{\mathcal{H}}$-submodule $K_{H}\left(\overline{\mathfrak{Z}}_{1}\right)$ of $K_{H}(\mathfrak{Z})$. The inclusions of these submodules induce $\mathcal{H}$-linear maps

(c) $\mathcal{H} \otimes \underline{\mathcal{H}} K_{H}\left(\underline{\Lambda}_{e}\right) \rightarrow K_{H}(\mathfrak{Z})$,

(d) $\mathcal{H} \otimes_{\mathcal{H}} K_{H}\left(\underline{\mathcal{B}}_{e}\right) \rightarrow K_{H}(\tilde{\mathfrak{Z}})$.

Theorem 7.11. The $\mathcal{H}$-linear maps 7.10(c),(d) are isomorphisms.

We show that $7.10(\mathrm{~d})$ is surjective. An equivalent statement is that the $\mathcal{H}$ submodule $\mathcal{M}$ of $K_{H}(\tilde{\mathfrak{Z}})$ generated by $K_{H}\left(\tilde{\mathfrak{Z}}_{1}\right)$ is the whole of $K_{H}(\tilde{\mathfrak{Z}})$. It is enough to show that, for any $w \in W_{*}$, the $R_{H}$-submodule $K_{H}\left(\tilde{\mathfrak{Z}}_{\leq w}\right)$ is contained in $\mathcal{M}$. We argue by induction on $l(w)$. When $w=1$, we have $\tilde{\mathfrak{Z}}_{\leq 1}=\tilde{\mathfrak{Z}}_{1}$ and the result is clear. Assume now that $w \neq 1$. We can find $i \in I$ such that $l\left(\sigma_{i} w\right)=l(w)-1$ (so that $\left.\sigma_{i} w \in W_{*}\right)$.

From 6.16(b), we see that $K_{H}\left(\tilde{\mathfrak{Z}}_{<w}\right)$ (identified with an $R_{H}$-submodule of $K_{H}(\tilde{\mathfrak{Z}})$ ) is equal to $\sum_{w^{\prime} \in W_{*} ; w^{\prime}<w} K_{H}\left(\tilde{\mathfrak{Z}}_{\leq w^{\prime}}\right)$ where each term of the sum is identified with an $R_{H}$-submodule of $K_{H}(\tilde{\mathfrak{Z}})$. Hence by the induction hypothesis, we have

(a) $K_{H}\left(\tilde{\mathfrak{Z}}_{\leq \sigma_{i} w}\right) \subset K_{H}\left(\tilde{\mathfrak{Z}}_{<w}\right) \subset \mathcal{M}$.

Let $\tilde{\xi} \in K_{H}\left(\tilde{\mathfrak{Z}}_{\leq w}\right)$. By $7.7(\mathrm{~b})$, there exist $\tilde{\xi}^{\prime} \in K_{H}\left(\tilde{\mathfrak{Z}}_{\leq \sigma_{i} w}\right)$ and $\tilde{\xi}^{\prime \prime} \in K_{H}\left(\tilde{\mathfrak{Z}}_{<w}\right)$ such that $\tilde{\xi}=\left(-\tilde{T}_{\sigma_{i}}-v^{-1}\right)\left(\tilde{\xi}^{\prime}\right)+\tilde{\xi}^{\prime \prime}$. Using (a), we see that $\tilde{\xi} \in \mathcal{M}$. This completes the induction. Thus, $7.10(\mathrm{~d})$ is surjective.

Next we note that $\mathcal{H}$ is a free (right) $\underline{\mathcal{H}}$-module of rank $\left|W / W^{*}\right|$; hence $\mathcal{H} \otimes_{\underline{\mathcal{H}}}$ $K_{H}\left(\underline{\mathcal{B}}_{e}\right)$ is isomorphic as an $R_{H}$-module to a direct sum of $\left|W / W^{*}\right|$ copies of $K_{H}\left(\underline{\mathcal{B}}_{e}\right)$ hence is free of rank $\left|W / W^{*}\right| \chi_{\underline{\mathcal{B}}_{e}}$ (see 1.14(a)) hence by 6.15(b), has the same rank as $K_{H}(\tilde{\mathfrak{Z}})$. (Note that $\left|W / W^{*}\right|=\left|W_{*}\right|$.) Thus, $7.10(\mathrm{~d})$ is a surjective $R_{H}$-linear map between two finitely generated free $R_{H}$-modules of the same rank. Hence it is necessarily an isomorphism. The proof that 7.10(a) is an isomorphism is entirely similar. The theorem is proved. 


\section{THE CASE WHERE $e$ IS REGULAR IN $\mathfrak{l}$}

8.1. In this section we preserve the setup of 6.1. We assume in addition that $e$ is a regular nilpotent element of $\mathfrak{l}$. Note that, in our case, $\Pi=\tilde{\Pi}$. (If $x \in \mathfrak{z}_{\mathfrak{p}}(f)$ and $e+x$ is nilpotent, then writing $x=x^{\prime}+x^{\prime \prime}$ with $x^{\prime} \in \mathfrak{z}_{\mathfrak{r}}(f), x^{\prime \prime} \in \mathfrak{z}_{\mathfrak{n}}(f)$, we have that $e+x^{\prime}$ is a nilpotent element of a cross section in $\mathfrak{l}$ to the regular nilpotent class in $\mathfrak{l}$, hence $x^{\prime}=0$ and $e+x=e+x^{\prime \prime} \in \tilde{\Pi}$.) It follows that

$$
\mathfrak{Z}=\tilde{\mathfrak{Z}} \text {. }
$$

We have $S=C$ where, as in $6.5, S$ is the connected centre of $L$. The fixed point set $\mathcal{B}_{e}^{H}=\Lambda_{e}^{H}$ is the finite set $\left\{\left(e, \mathfrak{b}_{w}\right) \mid w \in W_{*}\right\}$, where $\mathfrak{b}_{1}$ is the unique Borel subalgebra of $\mathfrak{p}$ such that $e \in \mathfrak{b}_{1}$ and, more generally, $\mathfrak{b}_{w}$ is the unique Borel subalgebra in $\mathbf{o}(w)$ that contains $\mathfrak{b}_{1} \cap \mathfrak{l}$. Hence Theorem 2.5(b) is applicable and yields:

(a) The $R_{H}$-bilinear pairing (:): $K_{H}\left(\mathcal{B}_{e}\right) \times K_{H}\left(\Lambda_{e}\right) \rightarrow R_{H}$ is perfect.

For any $w \in W_{*}, \mathfrak{Z}_{w}$ may be regarded as a $\mathbf{C}$-vector space of dimension $\operatorname{dim} \mathfrak{z}_{\mathfrak{n}}(f)$ with linear $H$-action (see $6.3(\mathrm{~b}))$. The origin of this vector space is the unique $(e, \mathfrak{b})$ such that $\mathfrak{b} \in \mathbf{o}(w)$ and $\mathfrak{b}$ contains $\mathfrak{b}_{1} \cap \mathfrak{l}$.

Let $\varpi: \mathfrak{g} \rightarrow \mathfrak{g}$ be as in 5.7 .

8.2. If $x \in \mathcal{X}$, then $C$ acts on the fibre at $\mathfrak{b}_{1}$ of the $G$-equivariant line bundle $L_{x}$ (since $\operatorname{Ad}(c) \mathfrak{b}_{1}=\mathfrak{b}_{1}$ for $c \in C$ ); this action is via a character $C \rightarrow \mathbf{C}^{*}$. This defines a homomorphism $\mathcal{X} \rightarrow \hat{C}$. For $i \in K, x=\alpha_{i}$ is in the kernel of this homomorphism. (Indeed, in this case the fibre of $L_{x}$ at $\mathfrak{b}_{1}$ is $\mathfrak{p}_{i} / \mathfrak{b}_{1}$ where $\mathfrak{p}_{i}$ is the unique parabolic subalgebra of type $i$ containing $\mathfrak{b}_{1}$. Now there exists $\xi \in \mathfrak{p}_{i} \cap \mathfrak{l}$ whose image in the line $\mathfrak{p}_{i} / \mathfrak{b}_{1}$ is non-zero. Since the adjoint action of $C$ on $\mathfrak{l}$ is trivial, our assertion follows.) Thus, setting $\mathcal{X}_{K}=\sum_{i \in K} \mathbf{Z} \alpha_{i} \subset \mathcal{X}$, we have an induced homomorphism

(a) $\mathcal{X} / \mathcal{X}_{K} \rightarrow \hat{C}$.

This is surjective. (Indeed, let $C^{\prime}$ be a maximal torus of $G$ such that $C \subset C^{\prime}$ and $\operatorname{Lie}\left(C^{\prime}\right) \subset \mathfrak{b}_{1}$. Then $\mathcal{X} \rightarrow \hat{C}$ factors as $\mathcal{X} \rightarrow \hat{C}^{\prime} \rightarrow \hat{C}$ where the first homomorphism, defined in the same way as $\mathcal{X} \rightarrow \hat{C}$, is an isomorphism, and the second homomorphism, given by restricting characters, is surjective, since $C$ is a closed subgroup of the torus $C^{\prime}$.) Since (a) is a homomorphism of abelian groups of the same finite rank and $\hat{C}$ is free, it follows that (a) induces an isomorphism

(b) $\left(\mathcal{X} / \mathcal{X}_{K}\right) /$ torsion $\stackrel{\sim}{\rightarrow} \hat{C}$.

8.3. If $x \in \mathcal{X}$, then $\mathbf{C}^{*}$ acts on the fibre at $\mathfrak{b}_{1}$ of the $G$-equivariant line bundle $L_{x}$ by $\operatorname{Ad}(\phi(\lambda))$ (since $\operatorname{Ad}(\phi(\lambda)) \mathfrak{b}_{1}=\mathfrak{b}_{1}$ for $\lambda \in \mathbf{C}^{*}$ ); this action is via a character $\lambda \rightarrow \lambda^{n}$ of $\mathbf{C}^{*}$ with $n \in \mathbf{Z}$. Now $x \mapsto n$ defines a homomorphism $\mu_{K}^{\prime}: \mathcal{X} \rightarrow \mathbf{Z}$. It is characterized by:

(a) $\mu_{K}^{\prime}\left(\alpha_{i}\right)=-2$ for all $i \in K$ and $\bigcap_{i \in K} \operatorname{Ker} \check{\alpha}_{i} \subset \operatorname{Ker} \mu_{K}^{\prime}$.

The assertion concerning $\mu_{K}^{\prime}\left(\alpha_{i}\right)$ is proved as in [L5, 12.21]. We prove the second assertion of (a). Let $x \in \bigcap_{i \in K} \operatorname{Ker} \check{\alpha}_{i}$. Then $L_{x}$ is the inverse image of a $G$ equivariant line bundle $L^{\prime}$ on the variety of all parabolic subalgebras of type $K$. Thus the fibre of $L_{x}$ at $\mathfrak{b}_{1}$ may be canonically identified with the fibre of $L^{\prime}$ at $\mathfrak{p}$, so that $P$ acts naturally on this fibre. Hence the homomorphism $\mathbf{C}^{*} \rightarrow \mathbf{C}^{*}$ associated to $x$ is a composition $\mathbf{C}^{*} \rightarrow P \rightarrow \mathbf{C}^{*}$ where the first map is $\lambda \rightarrow \phi(\lambda)$ and the second map is some homomorphism which is necessarily trivial on the derived subgroup of $P$ and hence on the image of the first map. Thus the composition is trivial and (a) is proved. 
It is easy to see that

(b) $\mu_{K}^{\prime}=\sum_{i \in K} c_{i} \check{\alpha}_{i}: \mathcal{X} \rightarrow \mathbf{Z}$, where $c_{i} \in \mathbf{Z}$.

Lemma 8.4. The $\underline{\mathcal{H}}$-module $K_{H}\left(\underline{\mathcal{B}}_{e}\right)=R_{H}$ can be described as follows. For $i \in K$, $\tilde{T}_{\sigma_{i}}$ acts as multiplication by $-v^{-1}$. For $x \in \mathcal{X}, \theta_{x}$ acts as multiplication by the character of $H$ which is the image of $x$ under the composition

$$
\mathcal{X} \rightarrow \hat{C} \times \hat{\mathbf{C}}^{*}=\hat{H}
$$

(The first map has two components: the first one is described in 8.2(a); the second one is $\mu_{K}^{\prime}$ as in 8.3(a).)

The assertion about $\theta_{x}$ follows from 8.2, 8.3. The assertion about $\tilde{T}_{\sigma_{i}}$ is deduced from the assertion about $\theta_{x}$ as in [L5, 12.21].

We can now restate 7.11 in the present case as follows.

Proposition 8.5. We have a canonical isomorphism of $\mathcal{H}$ - and $R_{H}$-modules

$$
\mathcal{H} \otimes_{\underline{\mathcal{H}}} R_{H} \stackrel{\sim}{\rightarrow} K_{H}(\mathfrak{Z})
$$

where $R_{H}$ is regarded as a left $\underline{\mathcal{H}}$-module as in 8.4 .

8.6. For an $H$-stable closed subvariety $Y \subset \Lambda_{e}$ such that the natural map (direct image) $K_{H}(Y) \rightarrow K_{H}\left(\Lambda_{e}\right)$ is injective, we shall often identify $K_{H}(Y)$ with its image in $K_{H}\left(\Lambda_{e}\right)$.

Let $p=\left\{\left(e, \mathfrak{b}_{1}\right)\right\} \subset \mathfrak{Z}_{1} \cap \mathcal{B}_{e}$. We shall denote $\mathfrak{O}_{p} \in K_{H}(p)$ again by $p$. Thus we have

$$
p \in K_{H}\left(\left\{\mathfrak{b}_{1}\right\}\right) \subset K_{H}\left(\mathfrak{Z}_{1}\right) \cap K_{H}\left(\mathcal{B}_{e}\right) \subset K_{H}\left(\Lambda_{e}\right)
$$

By 8.4, 7.10(b), we have

(a) $\tilde{T}_{i} p=-v^{-1} p$ for any $i \in K$.

In our case, $\mathfrak{Z}_{1}$ (see 7.10) becomes

(b) $\mathfrak{Z}_{1}=\left\{\left(y, \mathfrak{b}_{1}\right) \mid y \in e+\mathfrak{z}_{\mathfrak{n}}(f)\right\}$.

Thus, we have $\mathfrak{O}_{\mathfrak{Z}_{1}} \in K_{H}\left(\mathfrak{Z}_{1}\right) \subset K_{H}(\mathfrak{Z}) \subset K_{H}\left(\Lambda_{e}\right)$. From (b) we see that

(c) $p=\mathfrak{z}_{\mathfrak{n}}(f)^{*} \mathfrak{O}_{\mathfrak{Z}_{1}} \in K_{H}\left(\Lambda_{e}\right)$.

Let $w_{0}^{*}$ be the longest element of $W^{*}$ and let $w_{0 *}=w_{0} w_{0}^{*}$. Then $w_{0 *} \in W_{*}$ and $\mathbf{o}\left(w_{0 *}\right)$ is the set of all $\mathfrak{b} \in \mathcal{B}$ such that $\mathfrak{b}$ is opposed to some Borel subalgebra of $\mathfrak{p}$. Thus $\mathbf{o}\left(w_{0 *}\right)$ is the open $P$-orbit in $\mathcal{B}$. Hence $\mathfrak{Z}_{w_{0 *}}$ is open in $\mathfrak{Z}$.

Lemma 8.7. $\mathfrak{Z}_{w_{0 *}}=\left\{(e, \mathfrak{b}) \mid \mathfrak{b} \in \mathbf{o}\left(w_{0 *}\right), e \in \mathfrak{b}\right\}$.

Let $(y, \mathfrak{b}) \in \mathfrak{Z}_{w_{0 *}}$. We must prove that $y=e$. Since $\mathfrak{b} \in \mathbf{o}\left(w_{0 *}\right)$, the intersection $\mathfrak{b} \cap \mathfrak{p}$ is contained in a Levi subalgebra $\mathfrak{l}^{\prime}$ of $\mathfrak{p}$. Since $y \in \mathfrak{b}$, it follows that $y \in \mathfrak{l}^{\prime}$. Hence for some $u \in U$ we have $\operatorname{Ad}(u) y \in \mathfrak{l}$. Since $y \in e+\mathfrak{n}$, we have $\operatorname{Ad}(u) y \in e+\mathfrak{n}$. This implies (since $\operatorname{Ad}(u) y \in \mathfrak{l}, e \in \mathfrak{l}$ ) that $\operatorname{Ad}(u) y=e$. In particular, $y \in \operatorname{Ad}(G) e$. Since $y-e \in \mathfrak{z} \mathfrak{g}(f), y$ is in a cross section in $\mathfrak{g}$ to the $G$-orbit of $e$. This cross section intersects the $G$-orbit of $e$ exactly in $e$. Hence we must have $y=e$. The lemma is proved.

Lemma 8.8. (a) The two smooth submanifolds $\varpi\left(\mathfrak{Z}_{1}\right)$ and $\mathfrak{Z}_{w_{0 *}}$ of $\Lambda_{e}$ intersect transversally at the point $\varpi\left(e, \mathfrak{b}_{1}\right)$.

(b) The subsets $\mathcal{B}_{e}-\mathfrak{Z}_{w_{0 *}}$ and $\varpi\left(\mathfrak{Z}_{1}\right)$ of $\Lambda_{e}$ are disjoint. 
(Note that $\mathfrak{Z}$ is not stable under $\varpi: \Lambda_{e} \rightarrow \Lambda_{e}$.)

We prove $(\mathrm{a})$. We have $\mathfrak{b}_{1}=\left(\mathfrak{b}_{1} \cap \mathfrak{l}\right) \oplus\left(\mathfrak{b}_{1} \cap \mathfrak{n}\right)$ and $\varpi\left(\mathfrak{b}_{1} \cap \mathfrak{l}\right)=\mathfrak{b}_{1} \cap \mathfrak{l}$, while $\varpi(\mathfrak{n})$ is a complement of $\mathfrak{p}$ in $\mathfrak{g}$ (since $\varpi=-1$ on LieC) hence $\varpi\left(\mathfrak{b}_{1} \cap \mathfrak{n}\right) \cap \mathfrak{p}=0$ and $\varpi\left(\mathfrak{b}_{1}\right) \cap \mathfrak{p}=\mathfrak{b}_{1} \cap \mathfrak{l}$. This shows that $\varpi\left(\mathfrak{b}_{1}\right) \in \mathbf{o}\left(w_{0 *}\right)$ hence

$$
\varpi\left(e, \mathfrak{b}_{1}\right)=\left(e, \varpi\left(\mathfrak{b}_{1}\right)\right) \in \varpi\left(\mathfrak{Z}_{1}\right) \cap \mathfrak{Z}_{w_{0 *}} .
$$

Conversely, let $(y, \mathfrak{b}) \in \varpi\left(\mathfrak{Z}_{1}\right) \cap \mathfrak{Z}_{w_{0 *}}$. Since $(y, \mathfrak{b}) \in \mathfrak{Z}_{w_{0 *}}$, we have $y=e$ and $\mathfrak{b} \in \mathbf{o}\left(w_{0 *}\right)$. (See 8.7.) Since $(y, \mathfrak{b}) \in \varpi\left(\mathfrak{Z}_{1}\right)$, we have $\mathfrak{b}=\varpi\left(\mathfrak{b}_{1}\right)$. Thus, $(y, \mathfrak{b})=$ $\left(e, \varpi\left(\mathfrak{b}_{1}\right)\right)$. Hence $\varpi\left(\mathfrak{Z}_{1}\right) \cap \mathfrak{Z}_{w_{0 *}}=\left\{\left(e, \varpi\left(\mathfrak{b}_{1}\right)\right)\right\}$.

To check that the intersection is transversal we also need a computation in the tangent space to $\Lambda_{e}$ at $\left(e, \varpi\left(\mathfrak{b}_{1}\right)\right)$ which we omit. We note only that the smooth manifold $\Lambda_{e}$ is of pure dimension

$$
\operatorname{dim} \mathfrak{z}_{\mathfrak{g}}(f)-\operatorname{dim} \mathfrak{t}=2 \operatorname{dim} \mathfrak{z}_{\mathfrak{n}}(f)+\operatorname{dim} \mathfrak{z}_{\mathfrak{r}}(f)-\operatorname{dim} \mathfrak{t}=2 \operatorname{dim} \mathfrak{z}_{\mathfrak{n}}(f)
$$

and, by $6.3, \varpi\left(\mathfrak{Z}_{1}\right), \mathfrak{Z}_{w_{0 *}}$ are smooth submanifolds of pure dimension $\operatorname{dim} \mathfrak{z}_{\mathfrak{n}}(f)$ of $\Lambda_{e}$. This completes the proof of (a).

We prove (b). Assume that $(y, \mathfrak{b}) \in\left(\mathcal{B}_{e}-\mathfrak{Z}_{w_{0 *}}\right) \cap \varpi\left(\mathfrak{Z}_{1}\right)$. Since $(y, \mathfrak{b}) \in \mathcal{B}_{e} \cap$ $\varpi\left(\mathfrak{Z}_{1}\right)$, we must have $(y, \mathfrak{b})=\left(e, \varpi\left(\mathfrak{b}_{1}\right)\right)$. By $(\mathrm{a})$, we have $\left(e, \varpi\left(\mathfrak{b}_{1}\right)\right) \in \mathfrak{Z}_{w_{0 *}}$. This contradiction proves (b).

Lemma 8.9. Let $w \in W_{*}$. Let $d_{w}$ be the image of $\mathfrak{O}_{\mathfrak{Z}_{1}}$ under the composition $K_{H}(\mathfrak{Z}) \stackrel{\tilde{T}_{w}}{\longrightarrow} K_{H}(\mathfrak{Z}) \stackrel{j^{*}}{\longrightarrow} K_{H}\left(\mathfrak{Z}_{w_{0 *}}\right)$ where $j$ is the open imbedding $\mathfrak{Z}_{w_{0 *}} \subset \mathfrak{Z}$.

(a) There exists an element $\xi \in K_{H}\left(\mathfrak{Z}_{\leq w}\right)$ such that the restriction of $\xi$ to $K_{H}\left(\mathfrak{Z}_{w}\right)$ is $(-v)^{-l(w)} \mathfrak{O}_{\mathfrak{Z}_{w}}$ and $\xi=d_{w}$ in $K_{H}(\mathfrak{Z})$.

(b) If $w=w_{0 *}$, then $d_{w}=(-v)^{-l(w)} \mathfrak{O}_{\mathfrak{Z}_{w_{0 *}}}$.

(c) If $w \neq w_{0 *}$, then $d_{w}=0$.

We prove (a) by induction on $l(w)$. If $l(w)=0$, the result is trivial. Assume now that $l(w)>0$. We can find $i \in I$ such that $l\left(\sigma_{i} w\right)=l(w)-1$; then $w^{\prime}=\sigma_{i} w \in W_{*}$. We have

$$
\tilde{T}_{w} \mathfrak{O}_{\mathfrak{Z}_{1}}=\tilde{T}_{\sigma_{i}} \tilde{T}_{w^{\prime}} \mathfrak{O}_{\mathfrak{Z}_{1}}=-r_{i} \tilde{T}_{w^{\prime}} \mathfrak{O}_{\mathfrak{Z}_{1}}-v^{-1} \tilde{T}_{w^{\prime}} \mathfrak{O}_{\mathfrak{Z}_{1}}
$$

By the induction hypothesis, there exists $\xi^{\prime} \in K_{H}\left(\mathfrak{Z}_{\leq w^{\prime}}\right)$ such that the restriction of $\xi^{\prime}$ to $K_{H}\left(\mathfrak{Z}_{w^{\prime}}\right)$ is $(-v)^{-l\left(w^{\prime}\right)} \mathfrak{O}_{\mathfrak{Z}_{w^{\prime}}}$ and $\xi^{\prime}=\tilde{T}_{w^{\prime}} \mathfrak{O}_{\mathfrak{Z}_{1}}$ in $K_{H}(Z)$. Now $\xi^{\prime} \in K_{H}\left(\mathfrak{Z}_{\leq w}\right)$ hence we can apply to it $r_{i}: K_{H}\left(\mathfrak{Z}_{\leq w}\right) \rightarrow K_{H}\left(\mathfrak{Z}_{\leq w}\right)$. (Note that $\mathfrak{Z}_{\leq w}$ is $i$-saturated.) By 7.6(a), the image of $r_{i}\left(\xi^{\prime}\right)$ under the restriction $K_{H}\left(\mathfrak{Z}_{\leq w}\right) \rightarrow K_{H}\left(\mathfrak{Z}_{w}\right)$ is equal to $v^{-1}(-v)^{-l\left(w^{\prime}\right)} \mathfrak{O}_{\mathfrak{Z}_{w}}$. We set $\xi=-r_{i}\left(\xi^{\prime}\right)-v^{-1} \xi^{\prime} \in K_{H}\left(\mathfrak{Z}_{\leq w}\right)$. Then $\xi$ has the required properties. This proves (a). Now (b),(c) follow easily from (a). The lemma is proved.

Lemma 8.10. Let $w \in W_{*}$. Let $(:): \mathfrak{E}_{e} \times \mathfrak{E}_{e} \rightarrow \mathfrak{R}$ be as in 2.6.

(a) If $w=w_{0 *}$, then $\left(\tilde{T}_{w} \mathfrak{O}_{\mathfrak{Z}_{1}}: \varpi^{*} \mathfrak{O}_{\mathfrak{Z}_{1}}\right)=(-v)^{-l(w)}$.

(b) If $w \neq w_{0 *}$, then $\left(\tilde{T}_{w} \mathfrak{O}_{\mathfrak{Z}_{1}}: \varpi^{*} \mathfrak{O}_{\mathfrak{Z}_{1}}\right)=0$.

Let $\xi \in K_{H}\left(\mathfrak{Z}-\mathfrak{Z}_{w_{0 *}}\right) \subset K_{H}(\mathfrak{Z}) \subset \mathfrak{E}_{e}$. We first show that

(c) $\left(\xi: \varpi^{*} \mathfrak{O}_{\mathfrak{Z}_{1}}\right)=0$.

By [Th], the direct image map $K_{H}\left(\mathcal{B}_{e}-\mathfrak{Z}_{w_{0 *}}\right) \rightarrow K_{H}\left(\mathfrak{Z}-\mathfrak{Z}_{w_{0 *}}\right)$ is an isomorphism after extension of scalars to $\mathfrak{R}$, since $\left(\mathcal{B}_{e}-\mathfrak{Z}_{w_{0 *}}\right)^{H}=\left(\mathfrak{Z}-\mathfrak{Z}_{w_{0 *}}\right)^{H}$. Hence there exists $\xi^{\prime} \in K_{H}\left(\mathcal{B}_{e}-\mathfrak{Z}_{w_{0 *}}\right)$ and $f \in R_{H}-\{0\}$ such that $f \xi$ is the image of $\xi^{\prime}$ under this map. It is then enough to show that 
(d) $\left(\xi^{\prime}: \varpi^{*} \mathfrak{D}_{\mathfrak{Z}_{1}}\right)=0$.

Here (:) may be considered as the pairing in 2.4 with $\xi^{\prime}$ regarded as an element of $K_{H}\left(\mathcal{B}_{e}\right)$ and $\varpi^{*} \mathfrak{D}_{\mathfrak{Z}_{1}}$ regarded as an element of $K_{H}\left(\Lambda_{e}\right)$. But $\xi^{\prime}$ comes from an element in $K_{H}\left(\mathcal{B}_{e}-\mathfrak{Z}_{w_{0 *}}\right)$ and $\varpi^{*} \mathfrak{O}_{\mathfrak{Z}_{1}}$ comes from an element in $K_{H}\left(\varpi\left(\mathfrak{Z}_{1}\right)\right)$. To prove $(\mathrm{d})$, it is enough, by the definition of $(:)$, to show that $\mathcal{B}_{e}-\mathfrak{Z}_{w_{0 *}}$ and $\varpi\left(\mathfrak{Z}_{1}\right)$ are disjoint subsets of $\Lambda_{e}$. But this is known from 8.8(b). Thus, (d) and hence (c) are proved.

Now (b) follows from (c) (which is applicable in view of $8.9(\mathrm{c})$ ).

We prove (a). Let $w=w_{0 *}$. Let $V$ be the closure of $\mathfrak{Z}_{w}$ in $\mathcal{B}_{e}$. (See 8.7.) Let $[V]$ be the image of $\mathfrak{O}_{V} \in K_{H}(V)$ under the direct image map $K_{H}(V) \rightarrow K_{H}\left(\mathcal{B}_{e}\right)$. By $8.9(\mathrm{~b}), \tilde{T}_{w} \mathfrak{O}_{\mathfrak{Z}_{1}}$ and $(-v)^{-l(w)}[V]$ (regarded as elements of $K_{H}(\mathfrak{Z})$ ) have the same restriction to $K_{H}\left(\mathfrak{Z}_{w}\right)$, hence $\tilde{T}_{w} \mathfrak{O}_{\mathfrak{Z}_{1}}-(-v)^{-l(w)}[V]$ is the image of an element $\xi \in K_{H}\left(\mathfrak{Z}-\mathfrak{Z}_{w}\right)$. Applying (c) to $\xi$, we deduce that

$$
\left(\tilde{T}_{w} \mathfrak{O}_{\mathfrak{Z}_{1}}: \varpi^{*} \mathfrak{O}_{\mathfrak{Z}_{1}}\right)=\left((-v)^{-l(w)}[V]: \varpi^{*} \mathfrak{O}_{\mathfrak{Z}_{1}}\right) .
$$

Hence, to prove (a), it is enough to prove that

(e) $\left([V]: \varpi^{*} \mathfrak{O}_{\mathfrak{Z}_{1}}\right)=1$.

Here (:) may be considered as the pairing in 2.4 with $[V]$ regarded as an element of $K_{H}\left(\mathcal{B}_{e}\right)$ and $\varpi^{*} \mathfrak{O}_{\mathfrak{Z}_{1}}$ regarded as an element of $K_{H}\left(\Lambda_{e}\right)$. Then (e) follows immediately from 8.8(a),(b). The lemma is proved.

We can reformulate Lemma 8.10 in terms of $(\|)$ as follows.

Lemma 8.11. Let $w \in W_{*}$.

(a) If $w=w_{0 *}$, then $\left(\tilde{T}_{w_{0}}^{-1} \tilde{T}_{w} \mathfrak{O}_{\mathfrak{Z}_{1}} \| \mathfrak{O}_{\mathfrak{Z}_{1}}\right)=(-v)^{\nu-2 b(e)}(-v)^{-l(w)}$.

(b) If $w \neq w_{0 *}$, then $\left(\tilde{T}_{w_{0}}^{-1} \tilde{T}_{w} \mathfrak{O}_{\mathfrak{Z}_{1}} \| \mathfrak{O}_{\mathfrak{Z}_{1}}\right)=0$.

Lemma 8.12. Let $c=v^{-2 b(e)}$. For any $w, w^{\prime} \in W_{*}$ we have

(a) $\left(\tilde{T}_{w^{-1}}^{-1} \mathfrak{O}_{\mathfrak{Z}_{1}} \| \tilde{T}_{w^{\prime-1}}^{-1} \mathfrak{O}_{\mathfrak{Z}_{1}}\right)=\delta_{w, w^{\prime}} c$.

From 8.11(a) we have

$$
\left(\tilde{T}_{w_{0}^{*}}^{-1} \mathfrak{O}_{\mathfrak{Z}_{1}} \| \mathfrak{O}_{\mathfrak{Z}_{1}}\right)=(-v)^{\nu-2 b(e)}(-v)^{-\nu+l\left(w_{0}^{*}\right)} .
$$

Substituting here $\tilde{T}_{w_{0}^{*}}^{-1} \mathfrak{O}_{\mathfrak{Z}_{1}}=(-v)^{l\left(w_{0}^{*}\right)} \mathfrak{O}_{\mathfrak{Z}_{1}}$ (which follows from 8.6(a),(c)) we obtain

$$
\left((-v)^{l\left(w_{0}^{*}\right)} \mathfrak{O}_{\mathfrak{Z}_{1}} \| \mathfrak{O}_{\mathfrak{Z}_{1}}\right)=(-v)^{-2 b(e)}(-v)^{l\left(w_{0}^{*}\right)},
$$

so that (a) holds for $w=w^{\prime}=1$. Similarly, from 8.11 (b) we deduce that (a) holds for $w \neq 1$ and $w^{\prime}=1$.

We prove (a) by induction on $l\left(w^{\prime}\right)$. If $l\left(w^{\prime}\right)=0$, then (a) holds for any $w$ as we have just seen. Assume now that $l\left(w^{\prime}\right)>0$. We can find $i \in I$ such that $l\left(\sigma_{i} w^{\prime}\right)=l\left(w^{\prime}\right)-1$. We have $\sigma_{i} w^{\prime} \in W_{*}$. For $w \in W_{*}$ we have

$$
\begin{aligned}
& \left(\tilde{T}_{w^{-1}}^{-1} \mathfrak{O}_{\mathfrak{Z}_{1}} \| \tilde{T}_{w^{\prime-1}}^{-1} \mathfrak{O}_{\mathfrak{Z}_{1}}\right)=\left(\tilde{T}_{w^{-1}}^{-1} \mathfrak{O}_{\mathfrak{Z}_{1}} \| \tilde{T}_{\sigma_{i}}^{-1} \tilde{T}_{\left(\sigma_{i} w^{\prime}\right)^{-1}}^{-1} \mathfrak{O}_{\mathfrak{Z}_{1}}\right) \\
& =\left(\tilde{T}_{\sigma_{i}}^{-1} \tilde{T}_{w^{-1}}^{-1} \mathfrak{O}_{\mathfrak{Z}_{1}} \| \tilde{T}_{\left(\sigma_{i} w^{\prime}\right)^{-1}}^{-1} \mathfrak{O}_{\mathfrak{Z}_{1}}\right) .
\end{aligned}
$$

Assume first that $l\left(\sigma_{i} w\right)=l(w)-1$. Then $\tilde{T}_{\sigma_{i}}^{-1} \tilde{T}_{w^{-1}}^{-1}=\left(v^{-1}-v\right) \tilde{T}_{w^{-1}}^{-1}+\tilde{T}_{\left(\sigma_{i} w\right)^{-1}}^{-1}$ and $\sigma_{i} w \in W_{*}$ hence (b) becomes (using the induction hypothesis):

$$
\left(v^{-1}-v\right)\left(\tilde{T}_{w^{-1}}^{-1} \mathfrak{O}_{\mathfrak{Z}_{1}} \| \tilde{T}_{\left(\sigma_{i} w^{\prime}\right)^{-1}}^{-1} \mathfrak{O}_{\mathfrak{Z}_{1}}\right)+\left(\tilde{T}_{\left(\sigma_{i} w\right)^{-1}}^{-1} \mathfrak{O}_{\mathfrak{Z}_{1}} \| \tilde{T}_{\left(\sigma_{i} w^{\prime}\right)^{-1}}^{-1} \mathfrak{O}_{\mathfrak{Z}_{1}}\right)
$$

$$
=\left(v^{-1}-v\right) \delta_{w, \sigma_{i} w^{\prime}} c+\delta_{\sigma_{i} w, \sigma_{i} w^{\prime}} c \text {. }
$$


We cannot have $w=\sigma_{i} w^{\prime}$ since $l\left(\sigma_{i} w\right)=l(w)-1$ and $l\left(\sigma_{i} \sigma_{i} w^{\prime}\right)=l\left(\sigma_{i} w^{\prime}\right)+1$. Hence (c) becomes $\delta_{\sigma_{i} w, \sigma_{i} w^{\prime}} c=\delta_{w, w^{\prime}} c$.

Assume next that $l\left(\sigma_{i} w\right)=l(w)+1$ and $\sigma_{i} w \in W_{*}$. Then $w \neq w^{\prime}, \tilde{T}_{\sigma_{i}}^{-1} \tilde{T}_{w^{-1}}^{-1}=$ $\tilde{T}_{\left(\sigma_{i} w\right)^{-1}}^{-1}$ and (b) becomes (using the induction hypothesis):

$$
\left(\tilde{T}_{\left(\sigma_{i} w\right)^{-1}}^{-1} \mathfrak{O}_{\mathfrak{Z}_{1}} \| \tilde{T}_{\left(\sigma_{i} w^{\prime}\right)^{-1}}^{-1} \mathfrak{O}_{\mathfrak{Z}_{1}}\right)=\delta_{\sigma_{i} w, \sigma_{i} w^{\prime}} c=\delta_{w, w^{\prime}} c=0 .
$$

Finally, assume that $l\left(\sigma_{i} w\right)=l(w)+1$ and $\sigma_{i} w \notin W_{*}$. Then $\sigma_{i} w=w \sigma_{j}$ where $j \in K$. Note also that $w \neq \sigma_{i} w^{\prime}$. (If we had $w=\sigma_{i} w^{\prime}$, then $\sigma_{i} w=w^{\prime}$ would be in $W_{*}$.) We have

$$
\tilde{T}_{\sigma_{i}}^{-1} \tilde{T}_{w^{-1}}^{-1}=\tilde{T}_{\left(\sigma_{i} w\right)^{-1}}^{-1}=\tilde{T}_{\left(w \sigma_{j}\right)^{-1}}^{-1}=\tilde{T}_{w^{-1}}^{-1} \tilde{T}_{\sigma_{j}}^{-1}
$$

and (b) becomes (using the induction hypothesis):

$\left(\tilde{T}_{w^{-1}}^{-1} \tilde{T}_{\sigma_{j}}^{-1} \mathfrak{O}_{\mathfrak{Z}_{1}} \| \tilde{T}_{\left(\sigma_{i} w^{\prime}\right)^{-1}}^{-1} \mathfrak{O}_{\mathfrak{Z}_{1}}\right)=(-v)\left(\tilde{T}_{w^{-1}}^{-1} \mathfrak{O}_{\mathfrak{Z}_{1}} \| \tilde{T}_{\left(\sigma_{i} w^{\prime}\right)^{-1}}^{-1} \mathfrak{O}_{\mathfrak{Z}_{1}}\right)=(-v) \delta_{w, \sigma_{i} w^{\prime}} c=0$.

The lemma is proved.

Lemma 8.13. (a) The elements $f \tilde{T}_{w^{-1}}^{-1} \mathfrak{O}_{\mathfrak{Z}_{1}}$ (where $f$ runs over $\hat{C} \subset R_{C} \subset R_{H}$ and $w$ runs over $W_{*}$ ) form an $\mathcal{A}$-basis of $K_{H}(\mathfrak{Z})$.

(b) Let $f, f^{\prime} \in \hat{C} \subset R_{C} \subset R_{H}$ and let $w, w^{\prime} \in W_{*}$. We have

$$
\left(f \tilde{T}_{w^{-1}}^{-1} \mathfrak{O}_{\mathfrak{Z}_{1}} \| f^{\prime} \tilde{T}_{w^{\prime-1}}^{-1} \mathfrak{O}_{\mathfrak{Z}_{1}}\right)=f f^{\prime-1} \delta_{w, w^{\prime}} c .
$$

(c) For any $\xi, \xi^{\prime} \in K_{H}(\mathfrak{Z})$ we have $\left(\xi \| \xi^{\prime}\right) \in R_{H}$.

(d) Let $\xi \in K_{H}(\mathfrak{Z})$. We have $(\xi \| \xi)=1$ if and only if $\xi= \pm v^{b(e)} f \tilde{T}_{w^{-1}}^{-1} \mathfrak{O}_{\mathfrak{Z}_{1}}$ for some $f, w$ as in (a).

(a) is clear from 7.11 ; (b) follows immediately from 8.12 ; (c) follows immediately from (a),(b). (Note that in (c) we have apriori only $\left(\xi \| \xi^{\prime}\right) \in \mathfrak{R}$.)

We prove $(\mathrm{d})$. We can write uniquely

$$
\xi=\sum_{w, f} c_{w, f} v^{b(e)} f \tilde{T}_{w^{-1}}^{-1} \mathfrak{O}_{\mathfrak{Z}_{1}}
$$

where $w, f$ are as in (a) and $c_{w, f} \in \mathcal{A}$ are zero for all but finitely many $w, f$. Assume that $(\xi \| \xi)=1$. From (b) we deduce $\sum_{w, f} c_{w, f}^{2}=1$. It follows easily that there is a unique $w, f$ for which $c_{w, f} \neq 0$ and for that $w, f$ we have in fact $c_{w, f}= \pm 1$. Hence $\xi= \pm f \tilde{T}_{w^{-1}}^{-1} \mathfrak{O}_{\mathfrak{Z}_{1}}$. The converse is clear from (b). The lemma is proved.

Lemma 8.14. Let $\xi \in K_{H}(\mathfrak{Z})$ be an element such that $\tilde{T}_{\sigma_{i}} \xi=-v^{-1} \xi$ for all $i \in I$. Then there exists $g \in R_{H}$ such that $\xi=g \sum_{w \in W_{*}}(-v)^{l(w)} \tilde{T}_{w^{-1}}^{-1} \mathfrak{O}_{\mathfrak{Z}_{1}}$.

By $8.13(\mathrm{a})$, we can write uniquely $\xi=\sum_{w \in W_{*}} g_{w} \tilde{T}_{w^{-1}}^{-1} \mathfrak{O}_{\mathfrak{Z}_{1}}$ where $g_{w} \in R_{H}$. As in the proof of 8.12 , we see that

$$
\begin{gathered}
\tilde{T}_{\sigma_{i}}^{-1} \xi=\sum_{\begin{array}{c}
w \in W_{*} \\
l\left(\sigma_{i} w\right)=l(w)-1
\end{array}} g_{w}\left(\left(v^{-1}-v\right) \tilde{T}_{w^{-1}}^{-1} \mathfrak{O}_{\mathfrak{Z}_{1}}+\tilde{T}_{\left(\sigma_{i} w\right)^{-1}}^{-1} \mathfrak{O}_{\mathfrak{Z}_{1}}\right) \\
+\sum_{\begin{array}{c}
w \in W_{*} \\
l\left(\sigma_{i} w\right)=l(w)+1 \\
\sigma_{i} w \in W_{*}
\end{array}} g_{w} \tilde{T}_{\left(\sigma_{i} w\right)^{-1}}^{-1} \mathfrak{O}_{\mathfrak{Z}_{1}}-v \sum_{\begin{array}{c}
w \in W_{*} \\
l\left(\sigma_{i} w\right)=l(w)+1 \\
\sigma_{i} w \notin W_{*}
\end{array}} g_{w} \tilde{T}_{w^{-1}}^{-1} \mathfrak{O}_{\mathfrak{Z}_{1}} .
\end{gathered}
$$

The condition that this equals $-v \xi$ can be written in the form $g_{w}=-v g_{\sigma_{i} w}$ for any $w \in W_{*}$ such that $l\left(\sigma_{i} w\right)=l(w)-1$. It follows that $g_{w}=(-v)^{l(w)} g_{1}$ for all $w \in W_{*}$. The lemma is proved. 
Lemma 8.15. Let $z=\mathfrak{z}_{\mathfrak{n}}(f) \in R_{H}$. Then $\bar{z}^{\dagger}=\mathfrak{z}_{\mathfrak{n}}(f)^{*} \in R_{H}$. Let $\rho$ be the one dimensional representation of $C$ on $\Omega_{\mathfrak{z}_{\mathfrak{n}}(f)}$ (restriction from $H$ ). We have

(a) $\bar{z}^{\dagger}=(-1)^{b(e)} v^{b(e)+l\left(w_{0 *}\right)} \rho z$;

(b) Let $\hat{C}_{\geq}$(resp. $\hat{C}_{\leq}$) be the image of $\mathcal{X}_{\geq}$(resp. $\left.\mathcal{X}_{\leq}\right)$under $\mathcal{X} \rightarrow \hat{C}$ (see 8.2) and let $\hat{C}_{>}=\hat{C}_{\geq}-\{1\}$ (resp. $\left.\hat{C}_{<}=\hat{C}_{\leq}-\{1\}\right)$. We have

$$
z=\prod_{u=1}^{p}\left(1-v^{n_{u}} \tau_{u}\right)
$$

where for any $u$ we have $n_{u} \in \mathbf{Z}_{<0}$ and $\tau_{u} \in \hat{C}_{<}$.

We prove (a). The restriction of the one-dimensional $H$-module $\Omega_{\mathfrak{z}_{\mathfrak{n}}(f)}$ to $1 \times \mathbf{C}^{*}$ is $v^{t}$ for some $t \in \mathbf{Z}$. Using 3.1(a) and the equality $b(e)=\operatorname{dim} \mathfrak{z}_{\mathfrak{n}}(f)$, we see that we only have to prove that

(c) $t=b(e)+l\left(w_{0 *}\right)$.

Let $\mathfrak{n}^{\prime}$ be the nil-radical of the parabolic subalgebra opposed to $\mathfrak{p}$ and containing $\mathfrak{l}$. Now $\varpi: \mathfrak{g} \rightarrow \mathfrak{g}$ defines an isomorphism $\mathfrak{z}_{\mathfrak{n}}(f)^{\dagger}=\mathfrak{z}_{\mathfrak{n}^{\prime}}(f)$ as $H$-modules. Hence the restriction of $\Omega_{\mathfrak{z}_{\mathfrak{n}^{\prime}}(f)}$ to $1 \times \mathbf{C}^{*}$ is $v^{t}$. Since $f$ is regular nilpotent in $\mathfrak{l}$, the restriction of $\Omega_{\mathfrak{z}_{\mathrm{l}}(f)}$ to $1 \times \mathbf{C}^{*}$ is $v^{2 l\left(w_{0}^{*}\right)+2 r}$ where $r$ is the rank of $G$ (or of $L$ ). From the direct sum decomposition $\mathfrak{z}_{\mathfrak{g}}(f)=\mathfrak{z}_{\mathfrak{n}}(f) \oplus \mathfrak{z} \mathfrak{r}(f) \oplus \mathfrak{z}_{\mathfrak{n}}(f)$ we deduce

$$
\Omega_{\mathfrak{z}_{\mathfrak{g}}(f)}=\Omega_{\mathfrak{z}_{\mathfrak{n}}(f)} \otimes \Omega_{\mathfrak{z}_{\mathfrak{l}}(f)} \otimes \Omega_{\mathfrak{z}_{\mathfrak{n}^{\prime}}(f)}
$$

hence the restriction of $\Omega_{\mathfrak{z}_{\mathfrak{g}}(f)}$ to $1 \times \mathbf{C}^{*}$ is $v^{2 t+2 l\left(w_{0}^{*}\right)+2 r}$. In the restriction of $\Omega_{\mathfrak{g}}$ to $1 \times \mathbf{C}^{*}$, the action of $(1, \lambda)$ is by multiplication by $\lambda^{2 \operatorname{dim} \mathfrak{g}}$. (We use the fact that $\operatorname{Ad}(\phi(\lambda)): \mathfrak{g} \rightarrow \mathfrak{g}$ has determinant 1 since $\phi(\lambda)$ is in the derived group of $G$.) Since $\Omega_{\mathfrak{g}}=\Omega_{\mathfrak{z}_{\mathfrak{g}}(f)} \otimes \Omega_{\mathfrak{g} / \mathfrak{z} \mathfrak{g}(f)}$, it follows that the restriction of $\Omega_{\mathfrak{g} / \mathfrak{z} \mathfrak{g}(f)}$ to $1 \times \mathbf{C}^{*}$ is $v^{2 \operatorname{dim} \mathfrak{g}-2 t-2 l\left(w_{0}^{*}\right)-2 r}$. This restriction can be also computed directly, using the fact that $\mathfrak{g} / \mathfrak{z}_{\mathfrak{g}}(f)$ has a natural symplectic form (Kirillov) which is preserved up to a scalar $\lambda^{-2}$ by the action of $(1, \lambda) \in 1 \times \mathbf{C}^{*}$; namely, the restriction is $v^{\operatorname{dim}(\mathfrak{g} / \mathfrak{z} \mathfrak{g}(f))}$. We deduce that

$$
v^{\operatorname{dim} \mathfrak{g} / \mathfrak{z}_{\mathfrak{g}}(f)}=v^{2 \operatorname{dim} \mathfrak{g}-2 t-2 l\left(w_{0}^{*}\right)-2 r}
$$

from which (c) follows easily.

Since $\mathfrak{z}_{\mathfrak{n}}(f) \subset \bigoplus_{n \leq 0} \mathfrak{g}_{n}$, we see that $z$ is a product of factors of the form $1-\tau v^{m}$ where $\tau \in \hat{C}$ and $m \in \mathbf{Z}_{<0}$. We have in fact $\tau \in \hat{C}_{<0}$ since $\mathfrak{z}_{\mathfrak{n}}(f) \subset \mathfrak{n}$. This proves (b). The lemma is proved.

Lemma 8.16. (a) $z K_{H}(\mathfrak{Z}) \subset K_{H}\left(\mathcal{B}_{e}\right)$;

$$
\text { (b) } z^{\dagger} K_{H}\left(\Lambda_{e}\right) \subset K_{H}(\mathfrak{Z}) \text {. }
$$

We prove (a). Since $\mathfrak{O}_{\mathfrak{Z}_{1}}$ generates $K_{H}(\mathfrak{Z})$ as an $\mathcal{H}$-module (see 8.13(a)) and $z, \bar{z}^{\dagger}$ differ only by an invertible element of $R_{H}$ (see $8.15(\mathrm{a})$ ), it is enough to show that $\bar{z}^{\dagger} \mathfrak{O}_{\mathfrak{Z}_{1}} \in K_{H}\left(\mathcal{B}_{e}\right)$. But $\bar{z}^{\dagger} \mathfrak{O}_{\mathfrak{Z}_{1}}=p$ (see 8.6(c)) and clearly $p \in K_{H}\left(\mathcal{B}_{e}\right)$. This proves (a).

We prove (b). Let $\xi \in K_{H}\left(\Lambda_{e}\right)$. Since the canonical inclusion $K_{H}(\mathfrak{Z}) \subset K_{H}\left(\Lambda_{e}\right)$ becomes an equality after tensoring with $\mathfrak{R}$ over $R_{H}$, we see (using 8.13(a)) that

$$
g z^{\dagger} \xi=\sum_{w \in W_{*}} g_{w} \tilde{T}_{w^{-1}}^{-1} \mathfrak{O}_{\mathfrak{Z}_{1}} \in K_{H}(\mathfrak{Z})
$$


for some $g, g_{w}$ in $R_{H}$ with $g \neq 0$. Using 8.12 we have

$$
\left(\tilde{T}_{w^{-1}}^{-1} \mathfrak{O}_{\mathfrak{J}_{1}} \| g z^{\dagger} \xi\right)=g_{w}^{\dagger} v^{-2 b(e)}
$$

or equivalently

$$
g^{\dagger}\left(z \tilde{T}_{w^{-1}}^{-1} \mathfrak{O}_{\mathfrak{Z}_{1}} \| \xi\right)=g_{w}^{\dagger} v^{-2 b(e)}
$$

for any $w \in W_{*}$. By definition of $(\|)$ we have $\left(\xi_{1} \| \xi\right) \in R_{H}$ for any $\xi_{1} \in K_{H}\left(\mathcal{B}_{e}\right)$. (Recall that $\xi \in K_{H}\left(\Lambda_{e}\right)$.) By (a), we have $z \tilde{T}_{w^{-1}}^{-1} \mathfrak{O}_{\mathfrak{Z}_{1}} \in K_{H}\left(\mathcal{B}_{e}\right)$, hence

$$
\left(z \tilde{T}_{w^{-1}}^{-1} \mathfrak{O}_{\mathfrak{Z}_{1}} \| \xi\right) \in R_{H} \text {. }
$$

It follows that $g_{w}^{\dagger} v^{-2 b(e)} \in R_{H} g^{\dagger}$. Hence $g_{w}=u_{w} g$ for some $u_{w} \in R_{H}$. We see that

$$
g z^{\dagger} \xi=\sum_{w \in W_{*}} u_{w} g \tilde{T}_{w^{-1}}^{-1} \mathfrak{O}_{\mathfrak{Z}_{1}} .
$$

We can cancel $g$ in both sides and we obtain

$$
z^{\dagger} \xi=\sum_{w \in W_{*}} u_{w} \tilde{T}_{w^{-1}}^{-1} \mathfrak{O}_{\mathfrak{Z}_{1}}
$$

This shows that $z^{\dagger} \xi \in K_{H}(\mathfrak{Z})$. The lemma is proved.

8.17. Remark. From 8.16 we obtain a new proof and a strengthening (in the present case) of 3.5 .

As in the proof of $8.15(\mathrm{a})$ we have

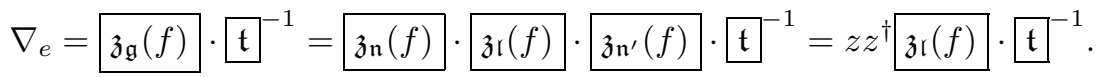

Now $\mathfrak{z} \mathfrak{r}(f) \cdot \mathfrak{t}^{-1} \in R_{H}$, by $3.2(\mathrm{a})$ applied to $\mathfrak{l}$ instead of $\mathfrak{g}$. It follows that $\nabla_{e}$ is divisible by $z z^{\dagger}$ in $R_{H}$.

Now 8.16(a),(b) shows that $z z^{\dagger} K_{H}\left(\Lambda_{e}\right) \subset K_{H}\left(\mathcal{B}_{e}\right)$. It follows that $\nabla_{e} K_{H}\left(\Lambda_{e}\right) \subset$ $K_{H}\left(\mathcal{B}_{e}\right)$, which is the statement of 3.5. Note that the statement $z z^{\dagger} K_{H}\left(\Lambda_{e}\right) \subset$ $K_{H}\left(\mathcal{B}_{e}\right)$ is stronger than the statement that $\nabla_{e} K_{H}\left(\Lambda_{e}\right) \subset K_{H}\left(\mathcal{B}_{e}\right)$.

Lemma 8.18. $\bar{z} \mathfrak{O}_{\Lambda_{e}}=\sum_{w \in W_{*}}(-v)^{l(w)} \tilde{T}_{w^{-1}}^{-1} \mathfrak{O}_{\mathfrak{Z}_{1}} \in K_{H}(\mathfrak{Z})$.

Let $\xi=\bar{z} \mathfrak{O}_{\Lambda_{e}}$ and let $\xi^{\prime}=\sum_{w \in W_{*}}(-v)^{l(w)} \tilde{T}_{w^{-1}}^{-1} \mathfrak{O}_{\mathfrak{Z}_{1}}$. By 8.15, 8.16, we have $\xi \in K_{H}(\mathfrak{Z})$. By 4.6 , we have $\tilde{T}_{\sigma_{i}} \xi=-v^{-1} \xi$ for all $i \in I$. Using 8.14, we deduce that $\xi=g \xi^{\prime}$ for some $g \in R_{H}$. From 8.13(b), we have

$$
\left(\mathfrak{O}_{\mathfrak{Z}_{1}} \| \xi\right)=g^{\dagger}\left(\mathfrak{O}_{\mathfrak{Z}_{1}} \| \xi^{\prime}\right)=g^{\dagger} v^{-2 b(e)} .
$$

We have

$$
\begin{aligned}
& \left(\mathfrak{O}_{\mathfrak{Z}_{1}} \| \xi\right)=\left(\mathfrak{O}_{\mathfrak{Z}_{1}} \| \bar{z} \mathfrak{O}_{\Lambda_{e}}\right)=\left(\bar{z}^{\dagger} \mathfrak{O}_{\mathfrak{Z}_{1}} \| \mathfrak{O}_{\Lambda_{e}}\right)=\left(p \| \mathfrak{O}_{\Lambda_{e}}\right) \\
& =(-v)^{\nu-2 b(e)}\left(p: \tilde{T}_{w_{0}} \varpi^{*} \mathfrak{O}_{\Lambda_{e}}\right)=(-v)^{\nu-2 b(e)}\left(p: \tilde{T}_{w_{0}} \mathfrak{O}_{\Lambda_{e}}\right)=(-v)^{-2 b(e)}\left(p: \mathfrak{O}_{\Lambda_{e}}\right) .
\end{aligned}
$$

(The last equality follows from 4.6.) Thus we have

$$
g^{\dagger}=\left(p: \mathfrak{O}_{\Lambda_{e}}\right) .
$$

Here (:) may be considered as the pairing in 2.4 with $p$ regarded as an element of $K_{H}\left(\mathcal{B}_{e}\right)$ and $\mathfrak{O}_{\Lambda_{e}}$ regarded as an element of $K_{H}\left(\Lambda_{e}\right)$. From the definition it is then obvious that $\left(p: \mathfrak{O}_{\Lambda_{e}}\right)=1$. Hence $g^{\dagger}=1$ and $g=1$. The lemma is proved. 
Lemma 8.19. We have

(a) $\quad z^{\dagger} v^{2 b(e)} \mathfrak{O}_{\Lambda_{e}}=(-1)^{l\left(w_{0 *}\right)} \rho \sum_{w \in W_{*}}(-v)^{l(w)-l\left(w_{0 *}\right)}(-v)^{b(e)} \tilde{T}_{w^{-1}}^{-1} \mathfrak{O}_{\mathfrak{Z}_{1}} \in K_{H}(\mathfrak{Z})$.

By $8.15(\mathrm{a}), 8.18$, the left hand side of (a) is

$$
\begin{aligned}
& (-1)^{b(e)} v^{-b(e)-l\left(w_{0 *}\right)} \rho \bar{z} v^{2 b(e)} \mathfrak{O}_{\Lambda_{e}} \\
& =(-1)^{b(e)} v^{-b(e)-l\left(w_{0 *}\right)} \rho v^{2 b(e)} \sum_{w \in W_{*}}(-v)^{l(w)} \tilde{T}_{w^{-1}}^{-1} \mathfrak{O}_{\mathfrak{Z}_{1}} .
\end{aligned}
$$

The lemma follows.

Proposition 8.20. $v^{2 b(e)} \mathfrak{O}_{\Lambda_{e}} \in \mathbf{B}_{\Lambda_{e}}^{ \pm}$.

By the argument in 5.14 we see that we only have to verify that

(a) $\left(v^{2 b(e)} \mathfrak{O}_{\Lambda_{e}} \| v^{2 b(e)} \mathfrak{O}_{\Lambda_{e}}\right) \in 1+v^{-1} R_{C}\left[\left[v^{-1}\right]\right]$.

By 8.19, the left hand side of (a) equals

$$
\left(z z^{\dagger}\right)^{-1} v^{2 b(e)}\left(\sum_{w \in W_{*}}(-v)^{l(w)-l\left(w_{0 *}\right)} \tilde{T}_{w^{-1}}^{-1} \mathfrak{O}_{\mathfrak{Z}_{1}} \| \sum_{w \in W_{*}}(-v)^{l(w)-l\left(w_{0 *}\right)} \tilde{T}_{w^{-1}}^{-1} \mathfrak{O}_{\mathfrak{Z}_{1}}\right)
$$

which, by 8.13 , is equal to

$$
\left(z z^{\dagger}\right)^{-1} \sum_{w \in W_{*}} v^{2 l(w)-2 l\left(w_{0 *}\right)}
$$

Using 8.15(b), we have $z z^{\dagger} \in 1+v^{-1} R_{C}\left[v^{-1}\right]$ hence $\left(z z^{\dagger}\right)^{-1} \in 1+v^{-1} R_{C}\left[\left[v^{-1}\right]\right]$. On the other hand, we have $\sum_{w \in W_{*}} v^{2 l(w)-2 l\left(w_{0 *}\right)} \in 1+v^{-1} \mathbf{Z}\left[v^{-1}\right]$ and (a) follows. The proposition is proved.

\section{A REVIEW OF [L4]}

9.1. We preserve the setup of 8.1. In addition we assume that $G$ is adjoint. Let $\mathcal{V}=\mathbf{R} \otimes \mathcal{X}$. We identify $\mathcal{X}$ with a subgroup of $\mathcal{V}$ by $x \mapsto 1 \otimes x$. We can regard $\check{\alpha}_{i}$ as R-linear forms on $\mathcal{V}$. Let $\mathcal{C}^{+}=\left\{x \in \mathcal{V} \mid \check{\alpha}_{i}(x)>0 \forall i \in I\right\}$. We extend the $W$-action on $\mathcal{X}$ to $\mathcal{V}$ in the obvious way. Let $\mathcal{I}$ be the subgroup of the group of affine transformations of $\mathcal{V}$ generated by $W$ and by the translations by elements of $\mathcal{X}$. The action of $\mathcal{I}$ on $\mathcal{V}$ is written as $(\omega, x) \mapsto{ }^{\omega} x$. Let $\mathcal{I}_{0}$ be the set of elements of $\mathcal{I}$ that are conjugate to $\sigma_{i}$ for some $i \in I$. For $\sigma \in \mathcal{I}_{0}$, let $h_{\sigma}$ be the fixed point set of $\sigma: \mathcal{V} \rightarrow \mathcal{V}$. Let $\mathfrak{F}$ be the set of hyperplanes in $\mathcal{V}$ of the form $h_{\sigma}$ for some $\sigma \in \mathcal{I}_{0}$. The set of points of $\mathcal{V}$ that are not contained in any hyperplane in $\mathcal{F}$ is a union of connected components called alcoves. Let $X$ be the set of alcoves. The set of points of $\mathcal{V}$ that belong to exactly one hyperplane in $\mathcal{F}$ is a union of connected components called faces. Let $S$ be the (finite) set of $\mathcal{I}$-orbits on the set of faces. If $s \in S$ and $F$ is a face in the $\mathcal{I}$-orbit of type $s$, we say that $F$ is of type $s$. Let opp :S $\rightarrow S$ be the involution defined by the following requirement: if $F$ is a face of type $s$, then $-F$ is a face of type $o p p(s)$.

For any alcove $A$ and any $s \in S$, there is a unique face $\delta_{s}(A)$ of type $s$ such that $\delta_{s}(A)$ is in the closure of $A$. For $s \in S$ and $A \in X$ we denote by $s A$ the unique alcove $\neq A$ such that $\delta_{s}(A)=\delta_{s}(s A)$. Then $A \mapsto s A$ is an involution of $X$. The maps $A \mapsto s A$ generate a subgroup of the group of permutations of $X$ which is a Coxeter group $\left(W^{a}, S\right)$ (an affine Weyl group). The permutation of $X$ corresponding to $w \in W^{a}$ is denoted by $A \mapsto w A$. 
For $h \in \mathcal{F}$ let $h^{+}$be the unique connected component of the complement of $h$ in $\mathcal{V}$ which contains $x+\mathcal{C}^{+}$for some $x \in \mathcal{V}$. For any $A \in X$ let $\mathcal{L}(A)$ be the set of all $s \in S$ such that, if $h$ is the hyperplane in $\mathcal{F}$ containing $\delta_{s}(A)$, then $A \subset h^{+}$.

Let $A_{0}^{+}$be the unique alcove contained in $\mathcal{C}^{+}$and having 0 in its closure.

For each $i \in I$ let $s_{i}^{0}$ be the type of the unique face of $A_{0}^{+}$that is contained in $\check{\alpha}_{i}^{-1}(0)$. Then $\sigma_{i} \mapsto s_{i}^{0}$ is an isomorphism of $W$ onto a subgroup of $W^{a}$ which carries $I$ bijectively onto a subset of $S$. We identify $W$ with its image in $W^{a}$.

The Hecke algebra (over $\mathcal{A}$ ) attached to this Coxeter group (as in $[L 5,1.4]$ is denoted in loc. cit. by $\mathcal{H}_{\mathrm{ad}}$. A homomorphism of algebras from our $\mathcal{H}$ to $\mathcal{H}_{\mathrm{ad}}$ is defined in [L5, 1.15-1.20] and is in fact an isomorphism. (We take $\epsilon=0$ in [L5, 1.15]). We use this to identify the two algebras. Thus, in $\mathcal{H}$ we have generators $\tilde{T}_{s}$ $(s \in S)$ which include the elements $\tilde{T}_{\sigma_{i}}$; we have $\tilde{T}_{s_{i}^{0}}=\tilde{T}_{\sigma_{i}}$ for $i \in I$; in $\mathcal{H}$ we also have elements $\tilde{T}_{w}$ for $w \in W^{a}$. Let ${ }^{-}: \mathcal{H} \rightarrow \mathcal{H}$ be the ring involution defined as in $[\mathrm{L} 5,1.5]$.

Let $\leq$ be the partial order on $X$ defined as in $[L 5,1.3]$.

9.2. Let $\mathcal{V}_{K}=\bigcap_{i \in K} \check{\alpha}_{i}^{-1}(0) \subset \mathcal{V}$. Let $\mathcal{F}^{K}$ be the set of all $h \in \mathcal{F}$ such that $h+\mathcal{V}_{K}=h$. The set of points of $\mathcal{V}$ that are not contained in any hyperplane in $\mathcal{F}^{K}$ is a union of connected components called $K$-alcoves. The subgroup $\mathcal{I}^{K}$ of $\mathcal{I}$ generated by $\left\{\sigma \in \mathcal{I}_{0} \mid h_{\sigma} \in \mathcal{F}^{K}\right\}$ acts transitively on the set of $K$-alcoves. Let $\Xi$ be a $K$-alcove. Let $X_{\Xi}$ be the set of alcoves that are contained in $\Xi$.

If $x \in \mathcal{X}$, then $x+\Xi$ is a $K$-alcove hence $x+\Xi=\omega^{-1} \Xi$ for a unique $\omega \in \mathcal{I}^{K}$. The map $x^{\prime} \mapsto^{\omega}\left(x+x^{\prime}\right)$ is then a homeomorphism $\gamma_{x}: \Xi \stackrel{\sim}{\rightarrow} \Xi$. If $A \in X_{\Xi}$, then $\gamma_{x}(A) \in X_{\Xi}$. As in [L4, 2.12], $x \mapsto \gamma_{x}$ is an action of $\mathcal{X}$ on $\Xi$ and an action of $\mathcal{X}$ on $X_{\Xi}$. The restrictions of these actions to $\mathcal{X}_{K}$ (see 8.2) are trivial, hence $x \mapsto \gamma_{x}$ can be regarded as an action of $\mathcal{X} / \mathcal{X}_{K}$ on $\Xi$ and an action of $\mathcal{X} / \mathcal{X}_{K}$ on $X_{\Xi}$.

Let $M^{\Xi}$ be the $\mathcal{A}$-module consisting of formal $\mathcal{A}$-linear combinations of alcoves $A \in X_{\Xi}$. The support of an element $m=\sum_{A \in X_{\Xi}} m_{A} A \in M^{\Xi}$ (with $m_{A} \in \mathcal{A}$ is the set $\operatorname{supp}(m)=\left\{A \in X_{\Xi} \mid m_{A} \neq 0\right\}$. Let $M_{c}^{\Xi}$ be the set of all $m \in M^{\Xi}$ such that $\operatorname{supp}(m)$ is finite.

Lemma 9.3. (a) There is a unique $\mathcal{H}$-module structure on the $\mathcal{A}$-module $M_{c}^{\Xi}$ such that for $s \in S$ and $A \in X_{\Xi}$ we have

$\tilde{T}_{s}(A)=s A$ if $s A \in X_{\Xi}, s \notin \mathcal{L}(A)$

$\tilde{T}_{s}(A)=s A+\left(v-v^{-1}\right) A$ if $s A \in X_{\Xi}, s \in \mathcal{L}(A)$;

$\tilde{T}_{s}(A)=v A$ if $s A \notin X_{\Xi}$.

(b) There is a unique $\mathcal{H}$-module structure on the $\mathcal{A}$-module $M_{c}^{\Xi}$ such that for $s \in S$ and $A \in X_{\Xi}$ we have

$\tilde{T}_{s}(A)=s A$ if $s A \in X_{\Xi}, s \notin \mathcal{L}(A)$

$\tilde{T}_{s}(A)=s A+\left(v-v^{-1}\right) A$ if $s A \in X_{\Xi}, s \in \mathcal{L}(A)$;

$\tilde{T}_{s}(A)=-v^{-1} A$ if $s A \notin X_{\Xi}$.

(a) is proved as in [L4, 4.7]. We shall deduce (b) from (a) as follows. Consider the involution of abelian groups $\psi: M_{c}^{\Xi} \rightarrow M_{c}^{\Xi}$ given by $\psi\left(v^{n} A\right)=(-v)^{-n} A$ for all $A \in X_{\Xi}, n \in \mathbf{Z}$. It is easy to check that $\tilde{T}_{s}: M_{c}^{\Xi} \rightarrow M_{c}^{\Xi}$ in (b) is equal to $\psi \tilde{T}_{s} \psi$ where $\tilde{T}_{s}$ is the map $M_{c}^{\Xi} \rightarrow M_{c}^{\Xi}$ in (a). Hence the braid group relations for the operators in (b) follow from the corresponding braid group relations for the operators in (a). The fact that the operators in (b) satisfy $\left(\tilde{T}_{s}+v^{-1}\right)\left(\tilde{T}_{s}-v\right)=0$ is obvious. The lemma is proved. 
9.4. We shall assume that $\Xi$ is the unique $K$-alcove that contains $A_{0}^{+}$. We shall denote by $\mathbf{M}_{c}$ the $\mathcal{H}$-module $M_{c}^{\Xi}$ described in 9.3(b).

Lemma 9.5. Let $\mu_{K}^{\prime}: \mathcal{X} \rightarrow \mathbf{Z}$ be as in 8.3. Let $x \in \mathcal{X}$. We have $\theta_{x} A_{0}^{+}=$ $(-v)^{\mu_{K}^{\prime}(x)} \gamma_{x}\left(A_{0}^{+}\right)$in $\mathbf{M}_{c}$.

See $[\mathrm{L} 4,7.5]$ for a closely related result.

Lemma 9.6. In $\mathbf{M}_{c}$ we have

(a) $\tilde{T}_{\sigma_{i}} A_{0}^{+}=-v^{-1} A_{0}^{+}$for all $i \in K$;

(b) $\tilde{T}_{w^{-1}}^{-1} A_{0}^{+}=w^{-1} A_{0}^{+} \in X_{\Xi}$ for all $w \in W_{*}$.

This follows easily from the definitions.

9.7. For $x \in \mathcal{X}$, the map $A \mapsto \gamma_{x} A$ in 9.2 extends to a $\mathcal{A}$-linear map $\mathbf{M}_{c} \rightarrow \mathbf{M}_{c}$ denoted again by $\gamma_{x}$. This map is in fact $\mathcal{H}$-linear (as in $[\mathrm{L} 4, \S 5]$ ). Thus, $\mathbf{M}_{c}$ becomes an $\mathcal{A}[\mathcal{X}]$-module.

We shall identify $\mathcal{X} / \mathcal{X}_{K}=\hat{C}$ by $8.2(\mathrm{~b})$. (In the present case, $\mathcal{X} / \mathcal{X}_{K}$ has no torsion since $G$ is adjoint.) We define an $R_{H}$-module structure on the $\mathcal{A}$-module $\mathbf{M}_{c}$ in which the generator $\tau \in \hat{C}$ of

$$
R_{H}=R_{C}\left[v, v^{-1}\right]=\mathcal{A}[\hat{C}]=\mathcal{A}\left[\mathcal{X} / \mathcal{X}_{K}\right]
$$

represented by $x \in \mathcal{X}$ acts by

$$
m \mapsto \tau m=(-1)^{\mu_{K}^{\prime}(x)} \gamma_{x}(m) .
$$

This again commutes with the $\mathcal{H}$-module structure.

(Note that the $R_{H}$-module structure and the $\mathcal{A}[\mathcal{X}]$-module structure on $\mathbf{M}_{c}$ are almost the same: they differ only by a sign; it will be convenient to use sometimes one, sometimes the other.)

Let $\tau \rightarrow \epsilon_{\tau}$ be the homomorphism $\hat{C} \rightarrow\{1,-1\}$ given by $\epsilon_{\tau}=(-1)^{\mu_{K}^{\prime}(x)}$ for $\tau, x$ as above. Then for any $B \in X_{\Xi}$ and $\tau \in \hat{C}$ we have $\tau B=\epsilon_{\tau} A$ for some $A \in X_{\Xi}$ which will be denoted $\tau * B$. Thus $\tau B=\epsilon_{\tau} \tau * B$ with $\tau * B \in X_{\Xi}$.

We define an $\mathcal{A}$-linear map $\mathcal{H} \otimes_{\mathcal{A}} R_{H} \rightarrow \mathbf{M}_{c}$ by

(a) $\xi \otimes f \mapsto(-v)^{-b(e)} \rho^{-1} f \xi\left(A_{0}^{+}\right)$

where $\xi \in \mathcal{H}, f \in R_{H}$.

Recall that $R_{H}$ is a module over the subalgebra $\underline{\mathcal{H}}$ of $\mathcal{H}$ (see 8.4). We show that for $\xi^{\prime} \in \underline{\mathcal{H}}, f \in R_{H}$, the element $\xi \xi^{\prime} \otimes f-\xi \otimes\left(\xi^{\prime} f\right)$ is mapped by (a) to 0 . It suffices to show that $\xi^{\prime}\left(f A_{0}^{+}\right)=\left(\xi^{\prime} f\right) A_{0}^{+}$for a set of algebra generators $\xi^{\prime}$ of $\underline{\mathcal{H}}$ and for $f \in R_{H}$. If $\xi^{\prime}=\theta_{x}$ where $x \in \mathcal{X}$, this follows from $9.5,8.4$; if $\xi^{\prime}=\tilde{T}_{\sigma_{i}}$ with $i \in K$, this follows from 9.6(a), 8.4. It follows that (a) factors through an $\mathcal{A}$-linear map

(b) $\mathcal{H} \otimes_{\underline{\mathcal{H}}} R_{H} \rightarrow \mathbf{M}_{c}$

(in fact an $\mathcal{H}$-linear and $R_{H}$-linear map).

9.8. Let $\kappa_{0}: \mathcal{V} \rightarrow \mathcal{V}$ be the involution $x \mapsto-w_{0}^{*} x$. Then $\kappa_{0}$ maps any alcove onto an alcove. Moreover, $\kappa_{0}(\Xi)=\Xi$. Hence $\kappa_{0}$ maps any alcove in $X_{\Xi}$ onto an alcove in $X_{\Xi}$. For any $x \in \mathcal{X}$ we have

(a) $\gamma_{x} \kappa_{0}=\kappa_{0} \gamma_{-x}$.

(See [L4, 2.3, 2.12(d)].) We define an involution of the abelian group $\mathbf{M}_{c}$ by $v^{n} A \mapsto$ $v^{-n} \kappa_{0}(A)$ for any $A \in X_{\Xi}$ and any $n \in \mathbf{Z}$. We denote it again by $\kappa_{0}$. This involution is $\mathcal{H}$-semilinear with respect to the ring involution of $\mathcal{H}$ given by 
(b) $\tilde{T}_{s} \mapsto \tilde{T}_{o p p(s)}^{-1}$ for $s \in S, v^{n} \mapsto v^{-n}$ for $n \in \mathbf{Z}$,

and $R_{H}$-semilinear with respect to $f \mapsto \bar{f}^{\dagger}$. (The first assertion is proved as in [L4, $6.5]$; the second assertion follows from (a).) Let

$$
\mathbf{e}_{0}=\sum_{w \in W_{*}}(-v)^{l(w)-l\left(w_{0 *}\right)}\left(w^{-1} A_{0}^{+}\right) \in \mathbf{M}_{c} .
$$

We have

$$
\kappa_{0}\left(\mathbf{e}_{0}\right)=\sum_{w \in W_{*}}(-v)^{-l(w)+l\left(w_{0 *}\right)}\left(w_{0}^{*} w^{-1} w_{0} A_{0}^{+}\right)=(-v)^{l\left(w_{0 *}\right)} \mathbf{e}_{0} .
$$

We consider the $\mathcal{A}$-algebra involution of $\mathcal{H}$ given by

(e) $\tilde{T}_{s} \mapsto \tilde{T}_{o p p(s)}$ for $s \in S$.

This carries $\theta_{x}$ to $\theta_{-w_{0} x}$ for all $x \in \mathcal{X}$.

Lemma 9.9. There exists a unique map $\mathbf{t}: \mathbf{M}_{c} \rightarrow z^{-1} \mathbf{M}_{c}$ (where $z^{-1} \mathbf{M}_{c}$ is regarded as a subspace of $\left.\left(\mathbf{M}_{c}\right)_{\mathfrak{R}}\right)$ such that $\mathbf{t}$ is $\mathcal{H}$-semilinear with respect to 9.8(e), $R_{H}$-semilinear with respect to $f \mapsto f^{\dagger}$, and such that $\mathbf{t}\left(\mathbf{e}_{0}\right)=\mathbf{e}_{0}$.

The uniqueness of $\mathbf{t}$ follows from the known fact that $\left(\mathbf{M}_{c}\right)_{\mathfrak{R}}$ is an irreducible $\mathcal{H}$-module and that the natural map $\mathbf{M}_{c} \rightarrow\left(\mathbf{M}_{c}\right)_{\mathfrak{R}}$ is injective (since $\mathbf{M}_{c}$ is free over $\left.R_{H}\right)$. The existence of $\mathbf{t}$ will be proved in 10.5.

Lemma 9.10. Let $\tilde{\mathbf{t}}: \mathbf{M}_{c} \rightarrow\left(z^{\dagger}\right)^{-1} \mathbf{M}_{c}$ be given by $\tilde{\mathbf{t}}(m)=\left(z^{\dagger}\right)^{-1} z \mathbf{t}(m)$. (Here $\left(z^{\dagger}\right)^{-1} \mathbf{M}_{c}$ is regarded as a subspace of $\left.\left(\mathbf{M}_{c}\right)_{\mathfrak{R}}\right)$. Let

$$
\tilde{\mathbf{e}}_{0}=(-v)^{l\left(w_{0 *}\right)} z \mathbf{e}_{0} \in \mathbf{M}_{c} .
$$

We have

$$
\begin{aligned}
& \text { (a) } \tilde{\mathbf{t}}\left(\tilde{\mathbf{e}}_{0}\right)=\tilde{\mathbf{e}}_{0} . \\
& \text { (b) } \kappa_{0}\left(\tilde{\mathbf{e}}_{0}\right)=(-1)^{l\left(w_{0 *}\right)}(-v)^{b(e)} \rho \tilde{\mathbf{e}}_{0} . \\
& \text { Indeed, } \tilde{\mathbf{t}}\left(\tilde{\mathbf{e}}_{0}\right)=\left(z^{\dagger}\right)^{-1} z \mathbf{t}\left((-v)^{l\left(w_{0 *}\right)} z \mathbf{e}_{0}\right)=(-v)^{l\left(w_{0 *}\right)} z \mathbf{e}_{0}=\tilde{\mathbf{e}}_{0}, \\
& \kappa_{0}\left(\tilde{\mathbf{e}}_{0}\right)=\kappa_{0}\left((-v)^{l\left(w_{0 *}\right)} z \mathbf{e}_{0}\right)=(-v)^{l\left(w_{0 *}\right)}(-v)^{-l\left(w_{0 *}\right)} \bar{z}^{\dagger} \mathbf{e}_{0}=(-v)^{-l\left(w_{0 *}\right)} \bar{z}^{\dagger} z^{-1} \tilde{\mathbf{e}}_{0} \\
& =(-1)^{l\left(w_{0 *}\right)}(-v)^{b(e)} \rho \tilde{\mathbf{e}}_{0} .
\end{aligned}
$$

The lemma is proved.

9.11. We define $\tilde{\mathbf{b}}: \mathbf{M}_{c} \rightarrow\left(z^{\dagger}\right)^{-1} \mathbf{M}_{c}$ by $\tilde{\mathbf{b}}(m)=(-1)^{l\left(w_{0 *}\right)}(-v)^{-b(e)} \rho \tilde{\mathbf{t}} \kappa_{0}(m)$.

We define $\mathbf{b}: \mathbf{M}_{c} \rightarrow z^{-1} \mathbf{M}_{c}$ by $\mathbf{b}(m)=(-v)^{-l\left(w_{0 *}\right)} \mathbf{t} \kappa_{0}(m)$.

Lemma 9.12. (a) $\tilde{\mathbf{b}}\left(\tilde{\mathbf{e}}_{0}\right)=\tilde{\mathbf{e}}_{0}$.

$$
\text { (b) } \mathbf{b}\left(\mathbf{e}_{0}\right)=\mathbf{e}_{0} \text {. }
$$

We have

$$
\begin{aligned}
\tilde{\mathbf{b}}\left(\tilde{\mathbf{e}}_{0}\right) & =(-1)^{l\left(w_{0 *}\right.}(-v)^{-b(e)} \rho \tilde{\mathbf{t}} \kappa_{0}\left(\tilde{\mathbf{e}}_{0}\right) \\
& =(-1)^{l\left(w_{0 *}\right.}(-v)^{-b(e)} \rho \tilde{\mathbf{t}}\left((-1)^{l\left(w_{0 *}\right)}(-v)^{b(e)} \rho \tilde{\mathbf{e}}_{0}\right)=\tilde{\mathbf{e}}_{0}, \\
\mathbf{b}\left(\mathbf{e}_{0}\right) & =(-v)^{-l\left(w_{0 *}\right)} \mathbf{t} \kappa_{0}\left(\mathbf{e}_{0}\right)=(-v)^{-l\left(w_{0 *}\right)} \mathbf{t}\left((-v)^{l\left(w_{0 *}\right)} \mathbf{e}_{0}\right)=\mathbf{e}_{0} .
\end{aligned}
$$

The lemma is proved. 
Lemma 9.13. (a) $\tilde{\mathbf{e}}_{0}$ is equal to $A_{0}^{+}$plus a finite $\mathcal{A}$-linear combination of alcoves $A \in X_{\Xi}, A<A_{0}^{+}$.

(b) Let $A_{0}^{!}=w_{0 *}^{-1} A_{0}^{+} \in X_{\Xi}$. Then $\mathbf{e}_{0}$ is equal to $A_{0}^{!}$plus a finite $\mathcal{A}$-linear combination of alcoves $A \in X_{\Xi}, A>A_{0}^{!}$.

(b) is obvious. We prove (a). We have $\mathbf{e}_{0}=(-v)^{-l\left(w_{0 *}\right)} A_{0}^{+}$plus a finite $\mathcal{A}$-linear combination of alcoves $A^{\prime} \in X_{\Xi}, A^{\prime}<A_{0}^{+}$. Moreover, by 8.15(b), we have $z$ equals 1 plus an $\mathcal{A}$-linear combination of elements in $\hat{C}_{<}$. One can show (see [L4, 2.10]) that, for any $A^{\prime} \in X_{\Xi}$ and any $\tau \in \hat{C}_{<}$, we have $\tau A^{\prime}<A^{\prime}$. This proves (a). The lemma is proved.

9.14. We can find (and we fix) $x_{1}, x_{2} \in \mathcal{X}$ such that, for any $w \in W_{*}$,

$$
{ }^{w^{-1}} A_{0}^{+} \subset \gamma_{x_{1}} A_{0}^{+}+\mathcal{C}^{+}, \quad w^{-1} A_{0}^{+} \subset \gamma_{x_{2}} A_{0}^{!}-\mathcal{C}^{+} .
$$

For any $A \in X_{\Xi}$ we define elements $p_{A}, p_{A}^{\prime}, q_{A}, q_{A}^{\prime}$ in $\mathbf{M}_{c}$ as follows. Assume first that $A={ }^{w^{-1}} A_{0}^{+}$for some $w \in W_{*}$. We have $A=y\left(\gamma_{x_{1}} A_{0}^{+}\right)$and $A=y^{\prime}\left(\gamma_{x_{2}} A_{0}^{!}\right)$for unique $y, y^{\prime} \in W^{a}$ and we set

$$
p_{A}=\tilde{T}_{y}\left(\gamma_{x_{1}} \tilde{\mathbf{e}}_{0}\right), p_{A}^{\prime}=\tilde{T}_{y^{-1}}^{-1}\left(\gamma_{x_{1}} \tilde{\mathbf{e}}_{0}\right), q_{A}=\tilde{T}_{y^{\prime}}\left(\gamma_{x_{2}} \mathbf{e}_{0}\right), q_{A}^{\prime}=\tilde{T}_{y^{\prime-1}}^{-1}\left(\gamma_{x_{2}} \mathbf{e}_{0}\right) .
$$

For general $A$, we can find a unique $x \in \mathcal{X}$ such that $A=\gamma_{x}\left(A^{\prime}\right)$ with $A^{\prime}={ }^{-1} A_{0}^{+}$ for some $w \in W_{*}$. Then $p_{A^{\prime}}, p_{A^{\prime}}^{\prime}, q_{A^{\prime}}, q_{A^{\prime}}^{\prime}$ are already defined and we set

$$
p_{A}=\gamma_{x} p_{A^{\prime}}, p_{A}^{\prime}=\gamma_{x} p_{A^{\prime}}^{\prime}, q_{A}=\gamma_{x} q_{A^{\prime}}, q_{A}^{\prime}=\gamma_{x} q_{A^{\prime}}^{\prime} .
$$

Using the definitions and 9.13, 9.12(a),(b) we see (as in [L4, 4.16]) that, for any $A \in X_{\Xi}$ :

(a) $p_{A}$ equals $A$ plus a finite $\mathcal{A}$-linear combination of alcoves $A_{1}<A$;

(b) $p_{A}^{\prime}$ equals $A$ plus a finite $\mathcal{A}$-linear combination of alcoves $A_{1}<A$;

(c) $q_{A}$ equals $A$ plus a finite $\mathcal{A}$-linear combination of alcoves $A_{1}>A$;

(d) $q_{A}^{\prime}$ equals $A$ plus a finite $\mathcal{A}$-linear combination of alcoves $A_{1}>A$;

(e) $\tilde{\mathbf{b}}\left(p_{A}\right)=p_{A}^{\prime}$;

(f) $\mathbf{b}\left(q_{A}\right)=q_{A}^{\prime}$.

9.15. The $\mathcal{H}$ structure of $\mathbf{M}_{c}$ extends in an obvious way to the whole of $M^{\Xi}$. We will denote $M^{\Xi}$ with this $\mathcal{H}$-module by $\mathbf{M}$. Similarly, the $\mathcal{A}[\mathcal{X}]$-module structure and the $R_{H}$-module structure on $\mathbf{M}_{c}$ extend in an obvious way to $\mathbf{M}$. Let $\mathbf{M}_{\leq}$ be the set of all $m \in \mathbf{M}$ such that $\operatorname{supp}(m)$ is bounded above under $\leq$. Let $\mathbf{M}_{\geq}$ be the set of all $m \in \mathbf{M}$ such that $\operatorname{supp}(m)$ is bounded below under $\leq$. Note that $\mathbf{M}_{\leq}, \mathbf{M}_{\geq}$are $\mathcal{H}$-submodules, $\mathcal{A}[\mathcal{X}]$-submodules and $R_{H}$-submodules of $\mathbf{M}$.

Let $R_{H, \leq}$ be the set of formal sums $\sum_{\tau} c_{\tau} \tau$ where $\tau$ runs over $\hat{C}$ and $c_{\tau} \in \mathcal{A}$ are zero except possibly for $\tau$ in some translate of $\hat{C}_{\leq}$in $\hat{C}$. Let $R_{H, \geq}$ be the set of formal sums $\sum_{\tau} c_{\tau} \tau$ where $\tau$ runs over $\hat{C}$ and $c_{\tau} \in \mathcal{A}$ are zero except possibly for $\tau$ in some translate of $\hat{C}_{\geq}$in $\hat{C}$. Then $R_{H, \leq}, R_{H, \geq}$ are naturally rings containing $R_{H}$, and $z, \bar{z}, z^{\dagger}, \bar{z}^{\dagger}$ are invertible elements of $R_{H, \leq}$ and $R_{H, \geq}$. (See 8.15(b).) Note that the $R_{H}$-module structure on $\mathbf{M}_{\leq}$extends naturally to an $R_{H, \leq- \text { module structure }}$ on $\mathbf{M}_{\leq}$and that the $R_{H}$-module structure on $\mathbf{M}_{\geq}$extends naturally to an $R_{H, \geq^{-}}$ module structure on $\mathbf{M}_{\geq}$.

Lemma 9.16. (a) There exists a unique map $\tilde{\mathbf{b}}^{\prime}: \mathbf{M}_{\leq} \rightarrow \mathbf{M}_{\leq}$which is $\mathcal{H}$-semilinear with respect to $\mathrm{t}^{-} \mathcal{H} \rightarrow \mathcal{H}, R_{H}$-semilinear with respect to $f \mapsto \bar{f}$, continuous (in the sense of $[\mathrm{L} 4,4.13]$ ), and satisfies $\tilde{\mathbf{b}}^{\prime}\left(\tilde{\mathbf{e}}_{0}\right)=\tilde{\mathbf{e}}_{0}$. 
(b) For any $A \in X_{\Xi}, \tilde{\mathbf{b}}^{\prime}(A)$ equals $A$ plus an (infinite) $\mathcal{A}$-linear combination of alcoves $A_{1}<A$.

(c) We have a commutative diagram

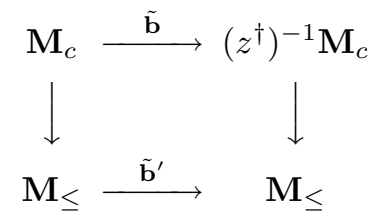

where the vertical maps are the natural imbeddings.

(d) $\tilde{\mathbf{b}}^{\prime 2}=1$.

(e) There exists a unique map $\mathbf{b}^{\prime}: \mathbf{M}_{\geq} \rightarrow \mathbf{M}_{\geq}$which is $\mathcal{H}$-semilinear with respect to ${ }^{-}: \mathcal{H} \rightarrow \mathcal{H}, R_{H^{-}}$-semilinear with respect to $\bar{f} \mapsto \bar{f}$, continuous (in the sense of $[\mathrm{L} 4,4.13])$, and satisfies $\mathbf{b}^{\prime}\left(\mathbf{e}_{0}\right)=\mathbf{e}_{0}$.

(f) For any $A \in X_{\Xi}, \mathbf{b}^{\prime}(A)$ equals $A$ plus an (infinite) $\mathcal{A}$-linear combination of alcoves $A_{1}>A$.

(g) We have a commutative diagram

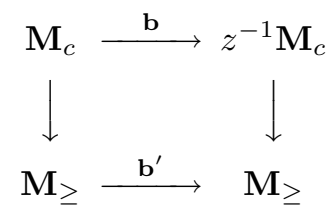

where the vertical maps are the natural imbeddings.

(h) $\mathbf{b}^{\prime 2}=1$.

Assume that for each $A \in X_{\Xi}$ we are given an element $L_{A} \in \mathbf{M}_{\leq}$so that $L_{\gamma_{x} A}=\gamma_{x} L_{A}$ for any $x \in \mathcal{X}$ and any $A$. Then there is a unique continuous map $L: \mathbf{M}_{\leq} \rightarrow \mathbf{M}_{\leq}$which is $R_{H^{-}}$-semilinear with respect to ${ }^{-}: R_{H} \rightarrow R_{H}$, and such that $L(A)=L_{A}$ for all $A \in X_{\Xi}$.

We apply this remark to the case where $L_{A}=\tilde{\mathbf{b}}(A) \in\left(z^{\dagger}\right)^{-1} \mathbf{M}_{c} \subset \mathbf{M}_{\leq}$for each $A$. The resulting map $\mathbf{M}_{\leq} \rightarrow \mathbf{M}_{\leq}$is denoted by $\tilde{\mathbf{b}}^{\prime}$. This map satisfies (c), hence it takes $\tilde{\mathbf{e}}_{0}$ to $\tilde{\mathbf{e}}_{0}$ (since $\tilde{\mathbf{b}}$ does). Hence it satisfies (a). Thus the existence part of (a) is established.

Now let $\tilde{\mathbf{b}}^{\prime}$ be any map as in (a). Using $9.15(\mathrm{a})$, any $A \in X_{\Xi}$ can be written uniquely as a series $A=\sum_{A^{\prime} \leq A} c_{A^{\prime}} p_{A^{\prime}}$ in $\mathbf{M}_{\leq}$where $c_{A^{\prime}} \in \mathcal{A}$ and $c_{A}=1$. Since $\tilde{\mathbf{b}}^{\prime}$

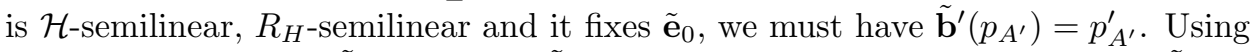
now the continuity of $\tilde{\mathbf{b}}^{\prime}$, we obtain $\tilde{\mathbf{b}}^{\prime}(A)=\sum_{A^{\prime}<A} \overline{{ }_{A_{A^{\prime}}}} p_{A^{\prime}}^{\prime}$. This shows that $\tilde{\mathbf{b}}^{\prime}(A)$ is uniquely determined for each $A$ and then, by the remark at the beginning of the proof, we see that $\tilde{\mathbf{b}}^{\prime}(m)$ is uniquely determined for each $m \in \mathbf{M}_{\leq}$. This completes the proof of (a) and (c). Now (b) follows from the formula $\tilde{\mathbf{b}}^{\prime}(A)=\sum_{A^{\prime} \leq A} \overline{c_{A^{\prime}}} p_{A^{\prime}}^{\prime}$ together with $9.15(\mathrm{~b})$.

Since $\tilde{\mathbf{b}}^{\prime 2}$ is $\mathcal{H}$-linear, $R_{H}$-linear, and it fixes $\tilde{\mathbf{e}}_{0}$, we have $\tilde{\mathbf{b}}^{\prime 2}\left(p_{A^{\prime}}\right)=p_{A^{\prime}}$. Since it is also continuous, we have $\tilde{\mathbf{b}}^{\prime 2}(A)=\sum_{A^{\prime} \leq A} c_{A^{\prime}} p_{A^{\prime}}$. Hence $\tilde{\mathbf{b}}^{\prime 2}(A)=A$. Using again the continuity of $\tilde{\mathbf{b}}^{\prime 2}$, we see that $\tilde{\mathbf{b}}^{\prime 2}(m)=m$ for all $m \in \mathbf{M}_{\leq}$. This proves (d). The proof of (e)-(h) is completely analogous to that of (a)-(d). (We replace $p_{A}, p_{A}^{\prime}$ by $q_{A}, q_{A}^{\prime}$.) The lemma is proved. 
9.17. Let $\mathfrak{m}=\left\{m=\sum_{A} m_{A} A \in \mathbf{M} \mid m_{A} \in \mathbf{Z}\left[v^{-1}\right] \forall A\right\}$. Let

$$
\mathfrak{m}_{c}=\mathfrak{m} \cap \mathbf{M}_{c}, \mathfrak{m}_{\leq}=\mathfrak{m} \cap \mathbf{M}_{\leq}, \mathfrak{m}_{\geq}=\mathfrak{m} \cap \mathbf{M}_{\geq} .
$$

Let $B \in X_{\Xi}$. Using 9.16(b),(d),(f),(h), we see as in [L4, 11.2] that

(a) there exists a unique element $B_{\leq} \in \mathfrak{m}_{\leq}$such that $B_{\leq}-B \in v^{-1} \mathfrak{m}_{\leq}$and such that $\tilde{\mathbf{b}}^{\prime}\left(B_{<}\right)=B_{<}$;

(b) we have $B_{\leq}=\sum_{A \in X_{\Xi} ; A \leq B} \tilde{\pi}_{A, B} A$ where $\tilde{\pi}_{A, B} \in v^{-1} \mathbf{Z}\left[v^{-1}\right]$ for all $A<B$ and $\tilde{\pi}_{B, B}=1$

(c) there exists a unique element $B_{\geq} \in \mathfrak{m}_{\geq}$such that $B \geq-B \in v^{-1} \mathfrak{m}_{\geq}$and such that $\mathbf{b}^{\prime}\left(B_{\geq}\right)=B_{\geq}$;

(d) we have $B_{\geq}=\sum_{A \in X_{\Xi} ; B \leq A} \pi_{B, A} A$ where $\pi_{B, A} \in v^{-1} \mathbf{Z}\left[v^{-1}\right]$ for all $B<A$ and $\pi_{B, B}=1$.

If $B=A_{0}^{!}$, then clearly $B \geq=\mathbf{e}_{0}$. (Note however, that $\tilde{\mathbf{e}}_{0}$ is not in general of the form $B \leq$ for some $B$.)

9.18. We define $\hat{\mathbf{b}}: \mathbf{M}_{c} \rightarrow\left(z^{\dagger}\right)^{-1} \mathbf{M}_{c}, \hat{\mathbf{b}}^{\prime}: \mathbf{M}_{\geq} \rightarrow \mathbf{M}_{\geq}$by

$$
\hat{\mathbf{b}}(m)=(-v)^{2 b(e)} \tilde{\mathbf{b}}(m)=\bar{z}^{\dagger}\left(z^{\dagger}\right)^{-1} \mathbf{b}(m), \quad \hat{\mathbf{b}}^{\prime}(m)=\bar{z}^{\dagger}\left(z^{\dagger}\right)^{-1} \mathbf{b}^{\prime}(m) .
$$

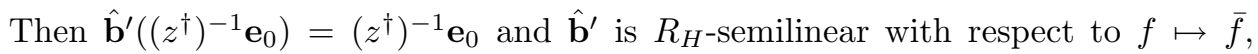
continuous. Moreover, $\hat{\mathbf{b}}^{\prime 2}=1$ and, for any $A \in X_{\Xi}, \mathbf{b}^{\prime}(A)$ equals $A$ plus a formal $\mathcal{A}$-linear combination of alcoves $A_{1}>A$. From this we deduce as in $[\mathrm{L} 4,11.2]$ that for any $B \in X_{\Xi}$ :

(a) there exists a unique element $\hat{B_{\geq}} \in \mathfrak{m}_{\geq}$such that $\hat{B_{\geq}}-B \in v^{-1} \mathfrak{m}_{\geq}$and such that $\hat{\mathbf{b}}^{\prime}\left(\hat{B_{\geq}}\right)=\hat{B_{\geq}}$

(b) we have $\hat{B}_{\geq}=\sum_{A \in X_{\Xi} ; B \leq A} \hat{\pi}_{B, A} A$ where $\hat{\pi}_{B, A} \in v^{-1} \mathbf{Z}\left[v^{-1}\right]$ for all $B<A$ and $\hat{\pi}_{B, B}=1$.

Note that $\left(z^{\dagger}\right)^{-1} B_{\geq}$satisfies the defining property (a) of $\hat{B_{\geq}}$, hence

$$
\hat{B_{\geq}}=\left(z^{\dagger}\right)^{-1} B_{\geq}
$$

9.19. We define $\check{\mathbf{b}}: \mathbf{M}_{c} \rightarrow z^{-1} \mathbf{M}_{c}, \check{\mathbf{b}}^{\prime}: \mathbf{M}_{\leq} \rightarrow \mathbf{M}_{\leq}$by

$$
\check{\mathbf{b}}(m)=(-v)^{2 l\left(w_{0 *}\right)} \mathbf{b}(m)=\bar{z} z^{-1} \tilde{\mathbf{b}}(m), \quad \check{\mathbf{b}}^{\prime}(m)=\bar{z} z^{-1} \tilde{\mathbf{b}}^{\prime}(m) .
$$

Then $\check{\mathbf{b}}^{\prime}\left(z^{-1} \tilde{\mathbf{e}}_{0}\right)=z^{-1} \tilde{\mathbf{e}}_{0}$ and $\check{\mathbf{b}}^{\prime}$ is $R_{H^{-}}$-semilinear with respect to $f \mapsto \bar{f}$, continuous. Moreover, $\check{\mathbf{b}}^{\prime 2}=1$ and, for any $A \in X_{\Xi}, \check{\mathbf{b}}^{\prime}(A)$ equals $A$ plus a formal $\mathcal{A}$-linear combination of alcoves $A_{1}<A$. From this we deduce as in $[\mathrm{L} 4,11.2]$ that for any $B \in X_{\Xi}$ :

(a) there exists a unique element $\check{B_{\leq}} \in \mathfrak{m}_{\leq}$such that $\check{B_{\leq}}-B \in v^{-1} \mathfrak{m}_{\leq}$and such that $\check{\mathbf{b}^{\prime}}\left(\check{B_{\leq}}\right)=\check{B_{\leq}}$

(b) we have $\check{B}_{\leq}=\sum_{A \in X \Xi ; \leq B} \check{\pi}_{A, B} A$ where $\check{\pi}_{A, B} \in v^{-1} \mathbf{Z}\left[v^{-1}\right]$ for all $A<B$ and $\check{\pi}_{B, B}=1$.

Note that $z^{-1} B_{\geq}$satisfies the defining property (a) of $\check{B_{\leq}}$, hence

$$
\check{B_{\leq}}=z^{-1} B_{\leq}
$$

9.20. We conjecture that

(a) $\tilde{\pi}_{A, B} \in \mathbf{N}\left[v^{-1}\right]$ for all $A \leq B$,

(b) $\pi_{B, A} \in \mathbf{N}\left[-v^{-1}\right]$ for all $B \leq A$. 


\section{Relating $\mathbf{B}_{\mathcal{B}_{e}}^{ \pm}, \mathbf{B}_{\Lambda_{e}}^{ \pm}$TO [L4]}

10.1. We preserve the setup of 9.1. In this section we give a proof of the existence part of Lemma 9.9 and describe a relationship between $\mathbf{B}_{\mathcal{B}_{e}}^{ \pm}, \mathbf{B}_{\Lambda_{e}}^{ \pm}$and the constructions in $\S 9$.

Lemma 10.2. There exists a (necessarily unique) isomorphism of $\mathcal{H}$ - and $R_{H^{-}}$ modules $\Phi: K_{H}(\mathfrak{Z}) \rightarrow \mathbf{M}_{c}$ such that $\Phi\left((-1)^{l\left(w_{0 *}\right)}(-v)^{b(e)} \rho \mathfrak{O}_{\mathfrak{Z}_{1}}\right)=A_{0}^{+}$. Under $\Phi$, the $\mathcal{A}$-basis $\left\{(-1)^{l\left(w_{0 *}\right)}(-v)^{b(e)} \rho f \tilde{T}_{w^{-1}}^{-1} \mathfrak{O}_{\mathfrak{Z}_{1}} \mid w \in W_{*}, f \in \hat{C}\right\}$ of $K_{H}(\mathfrak{Z})$ (see 8.13(a), 8.15) corresponds to the $\mathcal{A}$-basis $\left\{A \mid A \in X_{\Xi}\right\}$ of $\mathbf{M}_{c}$.

The uniqueness of $\Phi$ is clear since $K_{H}(\mathfrak{Z})$ is generated by $\mathfrak{O}_{\mathfrak{Z}_{1}}$ as a $\left(\mathcal{H}, R_{H}\right)$ bimodule. We prove the existence. We define $\Phi: K_{H}(\mathfrak{Z}) \rightarrow \mathbf{M}_{c}$ by

$$
\Phi(\xi)=(-1)^{l\left(w_{0 *}\right)} a_{1}\left(a_{2}(\xi)\right),
$$

where $a_{2}$ is the inverse of the isomorphism in 8.5 and $a_{1}$ is the map $9.7(\mathrm{~b})$. Then $\Phi$ is $\mathcal{H}$-linear and $R_{H}$-linear map. From the definitions, $\Phi$ carries the basis element $(-1)^{l\left(w_{0} *\right)}(-v)^{b(e)} \rho f \tilde{T}_{w^{-1}}^{-1} \mathfrak{O}_{\mathfrak{Z}_{1}}$ of $K_{H}(\mathfrak{Z})$ (where $w \in W_{*}, f \in \hat{C}$ ) onto $f \tilde{T}_{w^{-1}}^{-1} A_{0}^{+}=$ $f\left({ }^{w^{-1}} A_{0}^{+}\right)$. (The last equality follows from 9.6(b)). By $[\mathrm{L} 4,2.12(\mathrm{f})]$, the elements $f\left({ }^{w^{-1}} A_{0}^{+}\right)$above form an $\mathcal{A}$-basis of $\mathbf{M}_{c}$ which coincides with $\left\{A \mid A \in X_{\Xi}\right\}$. In particular, $\Phi$ is an isomorphism. The lemma is proved.

Lemma 10.3. $\Phi$ carries the element $z^{\dagger} v^{2 b(e)} \mathfrak{O}_{\Lambda_{e}} \in K_{H}(\mathfrak{Z})$ (see 8.16) to

$$
\mathbf{e}_{0}=\sum_{w \in W_{*}}(-v)^{l(w)-l\left(w_{0 *}\right) w^{-1}} A_{0}^{+} \in \mathbf{M}_{c} .
$$

This is a reformulation of 8.19 .

Lemma 10.4. Let $D_{\mathfrak{Z}}: K_{H}(\mathfrak{Z}) \rightarrow K_{H}(\mathfrak{Z})$ be Serre-Grothendieck duality on $\mathfrak{Z}$. For $m \in \mathbf{M}_{c}$ we have

$$
(-v)^{\nu-l\left(w_{0 *}\right)} v^{-2 b(e)} \bar{z}\left(z^{\dagger}\right)^{-1} \kappa_{0}(m)=\Phi\left(\tilde{T}_{w_{0}}^{-1} D_{\mathfrak{Z}}\left(\Phi^{-1}(m)\right)\right) .
$$

Both $m \mapsto(-v)^{\nu-l\left(w_{0 *}\right)} v^{-2 b(e)} \bar{z}\left(z^{\dagger}\right)^{-1} \kappa_{0}(m)$ and $m \rightarrow \Phi\left(\tilde{T}_{w_{0}}^{-1} D_{\mathfrak{Z}}\left(\Phi^{-1}(m)\right)\right)$ are

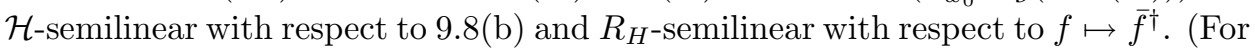
the second map this follows easily from [L5, 12.8, 9.7, 1.22], since $D_{\mathfrak{Z}}(\xi)=D_{\Lambda_{e}}(\xi)$ for $\xi \in K_{H}(\mathfrak{Z}) \subset K_{H}\left(\Lambda_{e}\right)$.) It follows that these two maps extend naturally to maps of the $\mathcal{H}$-module and $\mathfrak{R}$-vector space $\left(\mathbf{M}_{c}\right)_{\mathfrak{R}}$ into itself, which are $\mathcal{H}$-semilinear and $\mathfrak{R}$-semilinear in an obvious sense. Since the $\mathcal{H}$-module $\left(\mathbf{M}_{c}\right)_{\mathfrak{R}}$ is known to be (absolutely) irreducible and the natural map $\mathbf{M}_{c} \rightarrow\left(\mathbf{M}_{c}\right)_{\Re}$ is injective (since $\mathbf{M}_{c}$ is free over $R_{H}$ ), we see that it is enough to verify that our two maps have the same value on a particular non-zero vector of $\mathbf{M}_{c}$, which we take to be $\mathbf{e}_{0}$. (See 9.8(c).) We have

$$
\begin{aligned}
& \Phi\left(\tilde{T}_{w_{0}}^{-1} D_{\mathfrak{Z}}\left(\Phi^{-1}\left(\mathbf{e}_{0}\right)\right)\right)=\Phi\left(\tilde{T}_{w_{0}}^{-1} D_{\Lambda_{e}}\left(z^{\dagger} v^{2 b(e)} \mathfrak{O}_{\Lambda_{e}}\right)\right)=\Phi\left(\tilde{T}_{w_{0}}^{-1}\left(\bar{z} \mathfrak{O}_{\Lambda_{e}}\right)\right) \\
& =(-v)^{\nu} \bar{z} \Phi\left(\mathfrak{O}_{\Lambda_{e}}\right)=(-v)^{\nu} \bar{z}\left(z^{\dagger}\right)^{-1} v^{-2 b(e)} \mathbf{e}_{0},
\end{aligned}
$$

where we have used 10.3, 5.14(b), 5.14(c), 10.3. The lemma is proved.

10.5. We now prove the existence part of Lemma 9.9. Let $\tilde{\varpi}^{*}$ be the composition

$$
K_{H}(\mathfrak{Z}) \rightarrow K_{H}\left(\mathcal{B}_{e}\right) \stackrel{\varpi^{*}}{\longrightarrow} K_{H}\left(\mathcal{B}_{e}\right) \rightarrow z^{-1} K_{H}(\mathfrak{Z})
$$

where the first map is $\xi \mapsto z \xi$ (see $8.16(\mathrm{a})$ ) and the third map is $\xi \mapsto z^{-1} \xi$. Then $\tilde{\varpi}^{*}$ is $\mathcal{H}$-semilinear with respect to $9.8(\mathrm{e})$ (this is seen as in [L5, 12.6(a), 9.11]) 
and $R_{H}$-semilinear with respect to $f \mapsto f^{\dagger}$. We define $\mathbf{t}: \mathbf{M}_{c} \rightarrow z^{-1} \mathbf{M}_{c}$ as the composition

$$
\mathbf{M}_{c} \stackrel{\Phi^{-1}}{\longrightarrow} K_{H}(\mathfrak{Z}) \stackrel{\tilde{\varpi}^{*}}{\longrightarrow} z^{-1} K_{H}(\mathfrak{Z}) \stackrel{\Phi}{\longrightarrow} z^{-1} \mathbf{M}_{c}
$$

where the last $\Phi$ is the obvious extension of $K_{H}(\mathfrak{Z}) \stackrel{\Phi}{\rightarrow} \mathbf{M}_{c}$. We must only verify the equality

(a) $\mathbf{t}\left(\mathbf{e}_{0}\right)=\mathbf{e}_{0}$.

Since $\varpi^{*} \mathfrak{O}_{\Lambda_{e}}=\mathfrak{O}_{\Lambda_{e}}$ in $K_{H}\left(\Lambda_{e}\right)$ and $z^{\dagger} v^{2 b(e)} \mathfrak{O}_{\Lambda_{e}} \in K_{H}(\mathfrak{Z})$ (see 8.16(b)), we have $\tilde{\varpi}^{*}\left(z^{\dagger} v^{2 b(e)} \mathfrak{O}_{\Lambda_{e}}\right)=z^{-1} \varpi^{*}\left(z z^{\dagger} v^{2 b(e)} \mathfrak{O}_{\Lambda_{e}}\right)=z^{-1} z z^{\dagger} \varpi^{*}\left(v^{2 b(e)} \mathfrak{O}_{\Lambda_{e}}\right)=z^{\dagger} v^{2 b(e)} \mathfrak{O}_{\Lambda_{e}}$

in $K_{H}(\mathfrak{Z})$. We apply $\Phi$ and use 10.3 ; (a) follows. Lemma 9.9 is proved.

10.6. It will be convenient to extend $\tilde{\beta}, D_{\mathcal{B}_{e}}: K_{H}\left(\mathcal{B}_{e}\right) \rightarrow K_{H}\left(\mathcal{B}_{e}\right), \beta, D_{\Lambda_{e}}$ : $K_{H}\left(\Lambda_{e}\right) \rightarrow K_{H}\left(\Lambda_{e}\right)$, by the appropriate $R_{H}$-semilinearity, to $\mathfrak{R}$-semilinear operators $\tilde{\beta}, D, \beta, D: \mathfrak{E}_{e} \rightarrow \mathfrak{E}_{e}$. It will be also convenient to extend the operators

$$
\begin{aligned}
\kappa_{0}: \mathbf{M}_{c} & \rightarrow \mathbf{M}_{c}, \mathbf{t}: \mathbf{M}_{c} \rightarrow z^{-1} \mathbf{M}_{c}, \tilde{\mathbf{t}}: \mathbf{M}_{c} \rightarrow\left(z^{\dagger}\right)^{-1} \mathbf{M}_{c}, \\
\tilde{\mathbf{b}} & : \mathbf{M}_{c} \rightarrow\left(z^{\dagger}\right)^{-1} \mathbf{M}_{c}, \hat{\mathbf{b}}: \mathbf{M}_{c} \rightarrow\left(z^{\dagger}\right)^{-1} \mathbf{M}_{c},
\end{aligned}
$$

by the appropriate $R_{H}$-semilinearity, to $\Re$-semilinear operators

$$
\kappa_{0}, \mathbf{t}, \tilde{\mathbf{t}}, \tilde{\mathbf{b}}, \hat{\mathbf{b}}:\left(\mathbf{M}_{c}\right)_{\Re} \rightarrow\left(\mathbf{M}_{c}\right)_{\Re} .
$$

We extend $\Phi: K_{H}(\mathfrak{Z}) \rightarrow \mathbf{M}_{c}$ by $R_{H}$-linearity to an isomorphism $\Phi: \mathfrak{E}_{e} \stackrel{\sim}{\rightarrow}\left(\mathbf{M}_{c}\right)_{\Re}$. By 10.5, 9.10, we have

$$
\Phi \varpi^{*} \Phi^{-1}(m)=z\left(z^{\dagger}\right)^{-1} \mathbf{t}(m)=\tilde{\mathbf{t}}(m) .
$$

Using (a) and 10.4, we have

$$
\begin{gathered}
\Phi \tilde{\beta} \Phi^{-1}(m)=(-v)^{-\nu} \Phi \varpi^{*} \tilde{T}_{w_{0}}^{-1} D \Phi^{-1}(m)=(-v)^{-\nu} \Phi \varpi^{*} \Phi^{-1} \Phi \tilde{T}_{w_{0}}^{-1} D \Phi^{-1}(m) \\
=(-v)^{-\nu} \tilde{\mathbf{t}}\left((-v)^{\nu-l\left(w_{0 *}\right)} v^{-2 b(e)} \bar{z}\left(z^{\dagger}\right)^{-1} \kappa_{0}(m)\right)=(-1)^{l\left(w_{0 *}\right)}(-v)^{-b(e)} \rho \tilde{\mathbf{t}} \kappa_{0}(m) \\
=\tilde{\mathbf{b}}(m), \\
\Phi \beta \Phi^{-1}(m)=(-v)^{2 b(e)} \Phi \tilde{\beta} \Phi^{-1}(m)=(-v)^{2 b(e)} \tilde{\mathbf{b}}(m)=\hat{\mathbf{b}}(m), \text { hence } \\
\Phi \tilde{\beta} \Phi^{-1}=\tilde{\mathbf{b}}, \Phi \beta \Phi^{-1}=\hat{\mathbf{b}} .
\end{gathered}
$$

We now prove that

$$
\kappa_{0} \mathbf{t} \kappa_{0}=\mathbf{t}
$$

Since $\kappa_{0} \mathbf{t} \kappa_{0}$ has the same $\mathcal{H}$ - and $R_{H}$-semilinearity properties as $\mathbf{t}$, it suffices to show that $\kappa_{0} \mathbf{t} \kappa_{0}\left(\mathbf{e}_{0}\right)=\mathbf{e}_{0}$. This follows from 9.8(d) and 9.9.

The pairing $(\|): \mathfrak{E}_{e} \times \mathfrak{E}_{e} \rightarrow \mathfrak{R}$ can be transported under $\Phi: \mathfrak{E}_{e} \stackrel{\sim}{\rightarrow}\left(\mathbf{M}_{c}\right)_{\mathfrak{R}}$ to a pairing

(c) $(\|):\left(\mathbf{M}_{c}\right)_{\mathfrak{R}} \times\left(\mathbf{M}_{c}\right)_{\mathfrak{R}} \rightarrow \mathfrak{R}$.

The pairing (a) is $\mathfrak{R}$-linear in the first variable and $\mathfrak{R}$-semilinear (with respect to $\left.f \mapsto f^{\dagger}\right)$ in the second variable. Note that, by 8.13(b), for $A, B \in X_{\Xi}$ we have

(d) $(A \| B)=\epsilon_{\tau} \tau$ if $\tau * B=A$ for some $\tau \in \hat{C}$ (see 9.7); $(A \| B)=0$, otherwise. 
Proposition 10.7. (a) Let $\xi \in \mathbf{B}_{\mathcal{B}_{e}}^{ \pm}$. Then $\Phi(\xi)= \pm B_{\leq}$for a unique $B \in X_{\Xi}$. (Here we regard $\Phi(\xi) \in \mathbf{M}_{c} \subset \mathbf{M}_{\leq .}$)

(b) Let $\xi \in \mathbf{B}_{\Lambda_{e}}^{ \pm}$. Then $\Phi\left(z^{\dagger} \xi\right)= \pm B_{\geq}$for a unique $B \in X_{\Xi}$ (equivalently, $\Phi(\xi)= \pm \hat{B_{\geq}}$). (Here we regard $\Phi\left(z^{\dagger} \xi\right) \in \mathbf{M}_{c} \subset \mathbf{M}_{\geq}$.)

(c) Let $\xi \in K_{H}\left(\mathcal{B}_{e}\right)$. Assume that $\Phi(\xi) \in \mathbf{M}_{c}$ is equal to $\pm B_{\leq}$for some $B \in X_{\Xi}$. Then $\xi \in \mathbf{B}_{\mathcal{B}_{e}}^{ \pm}$.

(d) Let $\xi \in K_{H}\left(\Lambda_{e}\right)$. Assume that $\Phi\left(z^{\dagger} \xi\right) \in \mathbf{M}_{c}$ is equal to $\pm B_{\geq}$for some $B \in X_{\Xi}$. Then $\xi \in \mathbf{B}_{\Lambda_{e}}^{ \pm}$.

We prove (a). Since $\tilde{\beta}(\xi)=\xi$ and $\tilde{\mathbf{b}}=\Phi \tilde{\beta} \Phi^{-1}$, we have $\tilde{\mathbf{b}}(\Phi(\xi))=\Phi(\xi)$. Hence we have $\tilde{\mathbf{b}}^{\prime}(\Phi(\xi))=\Phi(\xi)$. Writing

$$
\xi=\sum_{f, w}(-1)^{l\left(w_{0 *}\right)}(-v)^{b(e)} \rho c_{f, w} f \tilde{T}_{w^{-1}}^{-1} \mathfrak{O}_{\mathfrak{Z}_{1}}
$$

(finite sum over $w \in W_{*}, f \in \hat{C}$ with $c_{f, w} \in \mathcal{A}$ ), see 8.13(a), and using the orthogonality formula $8.13(\mathrm{~b})$, we see that

$$
\partial(\xi \| \xi)=\sum_{f, w} c_{f, w}^{2} .
$$

By assumption, $(\xi \| \xi) \in 1+v^{-1} R_{C}\left[v^{-1}\right]$, hence $\partial(\xi \| \xi) \in 1+v^{-1} \mathbf{Z}\left[v^{-1}\right]$. Hence $\sum_{f, w} c_{f, w}^{2} \in 1+v^{-1} \mathbf{Z}\left[v^{-1}\right]$. It follows that $c_{f, w} \in v^{-1} \mathbf{Z}\left[v^{-1}\right]$ for all $(f, w)$ except one, for which $c_{f, w} \in \pm 1+v^{-1} \mathbf{Z}\left[v^{-1}\right]$. Thus, $\pm \Phi(\xi)$ satisfies the defining properties 9.17(a) of $B \leq$ for some $B \in X_{\Xi}$. This proves (a).

We prove (b). Since $\beta(\xi)=\xi$ and $\hat{\mathbf{b}}=\Phi \beta \Phi^{-1}$, we have $\hat{\mathbf{b}}(\Phi(\xi))=\Phi(\xi)$. Hence we have $\tilde{\mathbf{b}}^{\prime}(\Phi(\xi))=\Phi(\xi)$. Writing

$$
z^{\dagger} \xi=\sum_{f, w}(-1)^{l\left(w_{0 *}\right)}(-v)^{b(e)} \rho c_{f, w}^{\prime} f \tilde{T}_{w^{-1}}^{-1} \mathfrak{O}_{\mathfrak{Z}_{1}}
$$

(finite sum over $w \in W_{*}, f \in \hat{C}$ with $c_{f, w}^{\prime} \in \mathcal{A}$ ), see 8.13(a), and using the orthogonality formula $8.13(\mathrm{~b})$, we see that

$$
\partial\left(z^{\dagger} \xi \| z^{\dagger} \xi\right)=\sum_{f, w} c_{f, w}^{\prime}{ }^{2} .
$$

By assumption, $(\xi \| \xi) \in 1+v^{-1} R_{C}\left[\left[v^{-1}\right]\right]$. Hence

$$
\left(z^{\dagger} \xi \| z^{\dagger} \xi\right)=z z^{\dagger}(\xi|| \xi) \in 1+v^{-1} R_{C}\left[\left[v^{-1}\right]\right] .
$$

(See 8.15(b).) Since $z^{\dagger} \xi \in K_{H}(\mathfrak{Z})$ (see 8.16) we may use 8.13(c) to deduce that $\left(z^{\dagger} \xi \| z^{\dagger} \xi\right) \in R_{C}\left[v, v^{-1}\right]$. It follows that $\left(z^{\dagger} \xi \| z^{\dagger} \xi\right) \in 1+v^{-1} R_{C}\left[v^{-1}\right]$. Hence $\partial\left(z^{\dagger} \xi \| z^{\dagger} \xi\right) \in 1+v^{-1} \mathbf{Z}\left[v^{-1}\right]$.

It follows that $c_{f, w}^{\prime} \in v^{-1} \mathbf{Z}\left[v^{-1}\right]$ for all $(f, w)$ except one, for which $c_{f, w}^{\prime} \in$ $\pm 1+v^{-1} \mathbf{Z}\left[v^{-1}\right]$. Thus, there exists a unique $B \in X_{\Xi}$ such that

$$
\pm \Phi\left(z^{\dagger} \xi\right)-B \in v^{-1} \mathfrak{m} \cap \mathbf{M}_{c} .
$$

Now $\left(z^{\dagger}\right)^{-1} B-B \in v^{-1} \mathfrak{m}_{\geq}$and $\left(z^{\dagger}\right)^{-1}\left(v^{-1} \mathfrak{m}_{\geq}\right) \subset v^{-1} \mathfrak{m}_{\geq}$. Hence (e) implies

$$
\pm \Phi(\xi)-B \in v^{-1} \mathfrak{m}_{\geq}
$$

Thus, $\pm \Phi(\xi)$ satisfies the defining properties 9.18 (a) of $\hat{B_{\geq}}$for some $B \in X_{\Xi}$. This proves (b). 
We prove (c). Using 9.17(b) (where the sum is in our case known to be finite) and $10.6(\mathrm{~d})$, we see that

$$
\left(B_{\leq} \| B_{\leq}\right)=\sum_{A, \tau ; A \leq B, \tau * A \leq B} \tilde{\pi}_{\tau * A, B} \tilde{\pi}_{A, B} \epsilon_{\tau} \tau \in 1+v^{-1} R_{C}\left[v^{-1}\right] .
$$

Hence $(\xi|| \xi) \in 1+v^{-1} R_{C}\left[v^{-1}\right]$. From $\tilde{\mathbf{b}}\left(B_{\leq}\right)=B_{\leq}$we deduce that $\tilde{\beta}(\xi)=\xi$. This proves $(\mathrm{c})$. The proof of $(\mathrm{d})$ is similar to that of $(\mathrm{c})$. The proposition is proved.

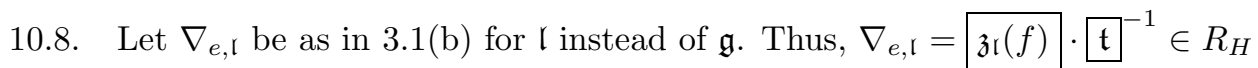
(see 3.2(a)). Since $f$ is regular nilpotent in $\mathfrak{l}$, we have $\nabla_{e, \mathfrak{l}} \in \mathcal{A}$.

Proposition 10.9. Let $\xi, \xi^{\prime} \in \mathbf{B}_{\Lambda_{e}}^{ \pm}$and $B, B^{\prime} \in X_{\Xi}$ be such that $\Phi(\xi)= \pm \hat{B_{\geq}}$, $\Phi\left(\xi^{\prime}\right)= \pm \hat{B_{>}^{\prime}}($ see 10.7(b)). Then

$\partial\left(\nabla_{e} \xi \| \xi^{\prime}\right)= \pm \nabla_{e, l} \sum_{A ; A \geq B ; A \geq B^{\prime}} \pi_{B, A} \pi_{B^{\prime}, A}$.

As in 8.17, we have $\nabla_{e}=z z^{\dagger} \nabla_{e, \mathfrak{l}}$. Hence

$$
\begin{aligned}
& \partial\left(\nabla_{e} \xi \| \xi^{\prime}\right)=\partial\left(z z^{\dagger} \nabla_{e, l} \xi \| \xi^{\prime}\right)=\partial \nabla_{e, \mathfrak{l}}\left(z^{\dagger} \xi \| z^{\dagger} \xi^{\prime}\right)= \pm \nabla_{e, l} \partial\left(z^{\dagger} \hat{B_{\geq}} \| z^{\dagger} \hat{B_{\geq}^{\prime}}\right) \\
& = \pm \nabla_{e, l} \partial\left(B_{\geq} \| B_{\geq}^{\prime}\right)= \pm \nabla_{e, l} \partial\left(\sum_{A ; A \geq B} \pi_{B, A} A \| \sum_{A^{\prime} ; A^{\prime} \geq B^{\prime}} \pi_{B^{\prime}, A^{\prime}} A^{\prime}\right) \\
& = \pm \nabla_{e, \mathfrak{l}} \sum_{A ; A \geq B ; A \geq B^{\prime}} \pi_{B, A} \pi_{B^{\prime}, A} .
\end{aligned}
$$

The proposition is proved.

Proposition 10.10. (a) Let $\xi, \xi^{\prime} \in \mathbf{B}_{\mathcal{B}_{e}}^{ \pm}$be such that $\pm \xi^{\prime} \notin \hat{C} \xi$. Let $B, B^{\prime} \in X_{\Xi}$ be such that $\Phi(\xi)= \pm B_{\leq}, \Phi\left(\xi^{\prime}\right)= \pm B_{\leq}^{\prime}($ see $10.7(a))$. We have $\left(\xi \| \xi^{\prime}\right) \in v^{-1} R_{C}\left[v^{-1}\right]$.

(b) Let $\xi, \xi^{\prime} \in \mathbf{B}_{\Lambda_{e}}^{ \pm}$be such that $\pm \xi^{\prime} \notin \hat{C} \xi$. Let $B, B^{\prime} \in X_{\Xi}$ be such that $\Phi(\xi)=$ $\pm \hat{B_{\geq}}, \Phi\left(\xi^{\prime}\right)= \pm \hat{B_{\geq}^{\prime}}\left(\right.$ see 10.7(b)). We have $\left(\xi \| \xi^{\prime}\right) \in v^{-1} R_{C}\left[\left[v^{-1}\right]\right]$.

We prove (a). We have

$$
\begin{aligned}
& \pm\left(\xi \| \xi^{\prime}\right)=\left(B_{\leq} \| B_{\leq}^{\prime}\right)=\left(\sum_{A} \tilde{\pi}_{A, B} A \| \sum_{A} \tilde{\pi}_{A, B^{\prime}} A\right) \\
& =\sum_{A, \tau} \epsilon_{\tau} \tilde{\pi}_{A * \tau, B} \tilde{\pi}_{A, B^{\prime}} \tau \in v^{-1} R_{C}\left[v^{-1}\right] .
\end{aligned}
$$

We prove (b). We have

$$
\begin{aligned}
& \pm z z^{\dagger}\left(\xi \| \xi^{\prime}\right)=\left(B_{\geq} \| B_{\geq}^{\prime}\right)=\left(\sum_{A} \pi_{B, A} A \| \sum_{A} \pi_{B^{\prime}, A} A\right) \\
& =\sum_{A, \tau} \epsilon_{\tau} \pi_{B, A * \tau} \pi_{B^{\prime}, A} \tau \in v^{-1} R_{C}\left[v^{-1}\right] .
\end{aligned}
$$

The proposition follows.

\section{INVERSION}

11.1. We preserve the setup of 9.1. Let $^{-}: \mathfrak{R} \rightarrow \mathfrak{R}$ be the field involution extending ${ }^{-}: R_{H} \rightarrow R_{H}$. 
Lemma 11.2. For $m, m^{\prime} \in\left(\mathbf{M}_{c}\right)_{\mathfrak{R}}$ we have

(a) $\left(\tilde{\mathbf{b}}(m) \| m^{\prime}\right)=\overline{\left(m \| \hat{\mathbf{b}}\left(m^{\prime}\right)\right)}$;

(b) $\left(\check{\mathbf{b}}(m) \| m^{\prime}\right)=\overline{\left(m \| \mathbf{b}\left(m^{\prime}\right)\right)}$.

(a) follows by transferring the identity [L5, 12.15] (which holds in our case with the same proof) and using 10.6. We shall deduce (b) from (a). We have $\check{\mathbf{b}}(m)=$ $\bar{z} z^{-1} \tilde{\mathbf{b}}(m)$ and $\hat{\mathbf{b}}\left(m^{\prime}\right)=\bar{z}^{\dagger}\left(z^{\dagger}\right)^{-1} \mathbf{b}\left(m^{\prime}\right)$ (see 9.19, 9.18). Hence

$$
\begin{aligned}
\left(\check{\mathbf{b}}(m) \| m^{\prime}\right) & =\bar{z} z^{-1}\left(\tilde{\mathbf{b}}(m) \| m^{\prime}\right)=\bar{z} z^{-1} \overline{\left(m \| \hat{\mathbf{b}}\left(m^{\prime}\right)\right)} \\
& =\bar{z} z^{-1} \overline{\left(m \| \bar{z}^{\dagger}\left(z^{\dagger}\right)^{-1} \mathbf{b}\left(m^{\prime}\right)\right)}=\overline{\left(m \| \mathbf{b}\left(m^{\prime}\right)\right)} .
\end{aligned}
$$

The lemma is proved.

11.3. We have

$$
\begin{aligned}
\tilde{\mathbf{b}}^{\prime}(A) & =\sum_{D \leq A} \tilde{r}_{D, A} D, \\
\mathbf{b}^{\prime}(A) & =\sum_{D \geq A} r_{A, D} D, \\
\hat{\mathbf{b}}^{\prime}(A) & =\sum_{D \geq A} \hat{r}_{A, D} D, \\
\check{\mathbf{b}}^{\prime}(A) & =\sum_{D \leq A} \check{r}_{D, A} D,
\end{aligned}
$$

where $\tilde{r}_{D, A}, r_{A, D}, \hat{r}_{A, D}, \check{r}_{D, A} \in \mathcal{A}$ are 1 for $D=A$. If $D \not \leq A$ we set $\tilde{r}_{D, A}=\check{r}_{D, A}=$ 0 ; if $A \not \leq D$ we set $r_{A, D}=\hat{r}_{A, D}=0$.

Lemma 11.4. For any $B \leq A$ in $X_{\Xi}$ we have

(a) $\tilde{r}_{B, A}=\overline{\hat{r}_{B, A}}$;

(b) $r_{B, A}=\overline{\tilde{r}_{B, A}}$.

We have $\tilde{\mathbf{b}}\left(\bar{z}^{\dagger} A\right)=\sum_{D} x_{D, A} D \in \mathbf{M}_{c}, \hat{\mathbf{b}}(z B)=\sum_{D} y_{B, D} D \in \mathbf{M}_{c}$, where $x_{D, A}, y_{B, D} \in \mathcal{A}$. By 11.2 , we have

$$
\left(\tilde{\mathbf{b}}\left(\bar{z}^{\dagger} A\right) \| B\right)=\overline{(A \| \hat{\mathbf{b}}(z B))}
$$

or equivalently $\left(\sum_{D} x_{D, A} D \| B\right)=\overline{\left(A \| \sum_{D} y_{B, D} D\right)}$. Using 10.6(d), we deduce

$$
\sum_{\tau \in \hat{C}} x_{\tau * B, A} \epsilon_{\tau} \tau=\sum_{\tau \in \hat{C}} \overline{y_{B, \tau^{-1} * A}} \epsilon_{\tau} \tau \in R_{H},
$$

hence

(c) $x_{\tau * B, A}=\overline{y_{B, \tau^{-1} * A}}$

for any $\tau \in \hat{C}$. We write $z=\sum_{\tau \in \hat{C}} z_{\tau} \tau$ (finite sum) with $z_{\tau} \in \mathcal{A}$. We have $\tilde{\mathbf{b}}\left(\bar{z}^{\dagger} A\right)=z^{\dagger} \tilde{\mathbf{b}}^{\prime}(A)$ hence $\sum_{D} x_{D, A} D=z^{\dagger} \sum_{D} \tilde{r}_{D, A} D$ and

(d) $x_{D, A}=\sum_{\tau} z_{\tau} \epsilon_{\tau} \tilde{r}_{\tau * D, A}$.

We have $\hat{\mathbf{b}}(z B)=\bar{z} \hat{\mathbf{b}}^{\prime}(B)$ hence $\sum_{D} y_{B, D} D=\bar{z} \sum_{D} \hat{r}_{B, D} D$,

(e) $y_{B, D}=\sum_{\tau} \bar{z}_{\tau} \epsilon_{\tau} \hat{r}_{B, \tau^{-1} * D}$.

Using now (c),(d),(e), we see that, for any $\tau^{\prime} \in \hat{C}$,

$$
\sum_{\tau} z_{\tau} \epsilon_{\tau} \tilde{r}_{\left(\tau \tau^{\prime}\right) * B, A}=\sum_{\tau} z_{\tau} \epsilon_{\tau} \overline{\hat{r}_{B,\left(\tau^{-1} \tau^{\prime-1}\right) * A}} .
$$

Clearly, $\hat{r}_{B,\left(\tau^{-1} \tau^{\prime-1}\right) * A}=\hat{r}_{\left(\tau \tau^{\prime}\right) * B, A}$. Hence

(f) $\sum_{\tau} z_{\tau} \epsilon_{\tau} \tilde{r}_{\left(\tau \tau^{\prime}\right) * B, A}=\sum_{\tau} z_{\tau} \epsilon_{\tau} \overline{\hat{r}_{\left(\tau \tau^{\prime}\right) * B, A}}$. 
Let

$$
g_{1}=\sum_{\tau} \epsilon_{\tau} \tilde{r}_{\tau * B, A} \tau^{-1} \in R_{H, \geq}, \quad g_{2}=\sum_{\tau} \epsilon_{\tau} \overline{\hat{r}_{\tau * B, A}} \tau^{-1} \in R_{H, \geq}
$$

$\left(R_{H, \geq}\right.$ as in 9.15.) Then (f) implies $z g_{1}=z g_{2}$. Since $z$ is invertible in $R_{H, \geq}$, it follows that $g_{1}=g_{2}$. This proves (a). The proof of (b) is entirely similar. The lemma is proved.

Lemma 11.5. The elements $\tilde{\pi}_{A, B}, \hat{\pi}_{B, A}, \check{\pi}_{A, B}, \pi_{B, A}$ in 9.17(b), 9.18(b), 9.19(b), 9.17(d), satisfy for $A \leq A^{\prime}$ in $X_{\Xi}$ :

(a) $\sum_{D ; A \leq D \leq A^{\prime}} \tilde{\pi}_{A, D} \hat{\pi}_{D, A^{\prime}}=\delta_{A, A^{\prime}}$,

(b) $\sum_{D ; A \leq D \leq A^{\prime}} \check{\pi}_{A, D} \pi_{D, A^{\prime}}=\delta_{A, A^{\prime}}$.

We prove (a). For any $A \leq B$ we define $\pi_{A, B}^{\prime}$ by the system of equations

$$
\sum_{D ; A \leq D \leq A^{\prime}} \tilde{\pi}_{A, D} \pi_{D, A^{\prime}}^{\prime}=\delta_{A, A^{\prime}}
$$

for any $A \leq A^{\prime}$ in $X_{\Xi}$. This system of equations has a unique solution and it clearly satisfies

$$
\pi_{A, A}^{\prime}=1, \pi_{A, B}^{\prime} \in v^{-1} \mathbf{Z}\left[v^{-1}\right] \text { for } A<B .
$$

It is then enough to show that $\pi_{A, B}^{\prime}=\hat{\pi}_{A, B}$ for all $A \leq B$. From the equalities

$$
\begin{gathered}
\hat{\mathbf{b}}^{\prime}\left(\sum_{A \in X_{\Xi} ; B \leq A} \hat{\pi}_{B, A} A\right)=\sum_{A \in X_{\Xi} ; B \leq A} \hat{\pi}_{B, A} A, \\
\hat{\mathbf{b}}^{\prime}(A)=\sum_{D \geq A} \hat{r}_{A, D} D
\end{gathered}
$$

(see 9.18, 11.3) we deduce

$$
\hat{\pi}_{B, D}=\sum_{A ; B \leq A \leq D} \overline{\hat{\pi}_{B, A}} \hat{r}_{A, D}
$$

or in matrix form, $\hat{\pi}=\overline{\hat{\pi}} \hat{r}$. In fact, this equation, together with the condition $\hat{\pi}_{A, A}=1, \hat{\pi}_{A, B} \in v^{-1} \mathbf{Z}\left[v^{-1}\right]$ for $A<B$, characterizes $\hat{\pi}$. Hence it is enough to show that the matrix $\pi^{\prime}$ satisfies the same equation as $\hat{\pi}$, that is, that

(c) $\pi^{\prime}=\overline{\pi^{\prime}} \hat{r}$.

From

$$
\tilde{\mathbf{b}}^{\prime}\left(\sum_{A ; A \leq B} \tilde{\pi}_{A, B} A\right)=\sum_{A ; A \leq B} \tilde{\pi}_{A, B} A, \quad \tilde{\mathbf{b}}^{\prime}(A)=\sum_{D \leq A} \tilde{r}_{D, A} D,
$$

(see $9.17,11.3$ ) we deduce

$$
\tilde{\pi}_{D, B}=\sum_{A ; D \leq A \leq B} \tilde{r}_{D, A} \overline{\tilde{\pi}_{A, B}}
$$

for any $D \leq B$, or in matrix form, $\tilde{\pi}=\tilde{r} \overline{\tilde{\pi}}$. Taking inverses gives $\pi^{\prime}=\overline{\pi^{\prime}} \tilde{r}^{-1}$. Hence to show (c), it is enough to show that

(d) $\tilde{r}^{-1}=\hat{r}$.

Since $\tilde{\mathbf{b}}^{\prime 2}=1$, we have the matrix equation $\tilde{r} \bar{r}=1$. Thus to prove (d), it is enough to show that $\hat{r}=\overline{\tilde{r}}$. But this follows from 11.4(a). This proves (a). The proof of (b) is entirely similar. The lemma is proved. 
11.6. The pairing $\mathbf{M}_{c} \times \mathbf{M}_{c} \rightarrow \mathcal{A}$ given by $m, m^{\prime} \mapsto \partial\left(m \| m^{\prime}\right)$ (where $\left(m \| m^{\prime}\right)$ is as in 10.6(c)) can be extended to a pairing

$$
\mathbf{M}_{\leq} \times \mathbf{M}_{\geq} \rightarrow \mathcal{A}
$$

given by $m, m^{\prime} \mapsto \sum_{A} m_{A} m_{A}^{\prime}$ for $m=\sum_{A} m_{A} A, m^{\prime}=\sum_{A^{\prime}} A^{\prime}$ with $m_{A}, m_{A}^{\prime} \in \mathcal{A}$. (See 10.6(d).) We denote this pairing by $\partial(\|)$ even though $\left(m \| m^{\prime}\right)$ is not defined. With this notation we can reformulate 11.5 as follows.

Lemma 11.7. For $A, A^{\prime} \in X_{\Xi}$ we have

(a) $\partial\left(A_{\leq} \| \hat{A_{\geq}^{\prime}}\right)=\delta_{A, A^{\prime}}$;

(b) $\partial\left(\tilde{A_{\leq}} \| A_{\geq}^{\prime}\right)=\delta_{A, A^{\prime}}$.

With the notation of 11.5 , we see that (a) is equivalent to the matrix equation $\hat{\pi} \tilde{\pi}=1$. This is equivalent to the matrix equation $\tilde{\pi} \hat{\pi}=1$, which is just 11.5 (a). A similar argument gives (b).

\section{Boundedness of POLES}

12.1. We preserve the setup of 9.1.

Lemma 12.2. For any $m, m^{\prime} \in\left(\mathbf{M}_{c}\right)_{\mathfrak{R}}$ we have

(a) $\left(m \| \tilde{\mathbf{t}}\left(m^{\prime}\right)\right)=\left(\tilde{\mathbf{t}}(m) \| m^{\prime}\right)^{\dagger}$,

(b) $\left(m \| \mathbf{t}\left(m^{\prime}\right)\right)=\left(\mathbf{t}(m) \| m^{\prime}\right)^{\dagger}$.

From 5.6(a) it follows easily that $\left(\varpi^{*} \xi \| \xi^{\prime}\right)=\left(\xi \| \varpi^{*} \xi^{\prime}\right)^{\dagger}$ for $\xi, \xi^{\prime} \in \mathfrak{E}_{e}$. This implies, by 10.6(a) that (b) holds. Now (b) follows from (a) using the equality

(c) $z\left(z^{\dagger}\right)^{-1} \mathbf{t}(m)=\tilde{\mathbf{t}}(m)$.

Lemma 12.3. The maps $\tilde{\mathbf{t}}, \mathbf{t}:\left(\mathbf{M}_{c}\right)_{\mathfrak{R}} \rightarrow\left(\mathbf{M}_{c}\right)_{\mathfrak{R}}$ are involutions.

Using 10.6(a) we have $\tilde{\mathbf{t}}^{2}=\Phi \varpi^{*} \Phi^{-1} \Phi \varpi^{*} \Phi^{-1}=1$ since $\varpi^{*}=1$. By 12.2(c),

$$
\tilde{\mathbf{t}}^{2}(m)=\tilde{\mathbf{t}}\left(z\left(z^{\dagger}\right)^{-1} \mathbf{t}(m)\right)=z^{-1} z^{\dagger} \tilde{\mathbf{t}}(\mathbf{t}(m))=\mathbf{t}^{2}(m) .
$$

Thus, $\mathbf{t}^{2}=1$. The lemma is proved.

12.4. Define $\mathbf{t}_{1}: \mathbf{M}_{c} \rightarrow \mathbf{M}_{c}$ by $\mathbf{t}_{1}(m)=z \mathbf{t}(m)=z^{\dagger} \tilde{\mathbf{t}}(m)$.

For $m, m^{\prime} \in \mathbf{M}_{c}$ we have (using 12.2, 12.3)

$$
\left(\mathbf{t}_{1}(m) \| \mathbf{t}_{1}\left(m^{\prime}\right)\right)=z z^{\dagger}\left(\mathbf{t}(m) \| \mathbf{t}\left(m^{\prime}\right)\right)=z z^{\dagger}\left(\mathbf{t}^{2}(m) \| m^{\prime}\right)^{\dagger}=z z^{\dagger}\left(m \| m^{\prime}\right)^{\dagger} .
$$

In particular, $\left(\mathbf{t}_{1}(A) \| \mathbf{t}_{1}(A)\right)=z z^{\dagger}$. Hence $\partial\left(\mathbf{t}_{1}(A) \| \mathbf{t}_{1}(A)\right)=\partial\left(z z^{\dagger}\right)$. Setting $\mathbf{t}_{1}(A)=\sum_{D} g_{D, A} D$, we can rewrite this, using 10.6(d):

$$
\sum_{D} g_{D, A}^{2}=\partial\left(z z^{\dagger}\right) \in 1+v^{-1} \mathbf{Z}\left[v^{-1}\right] .
$$

It follows that, for fixed $A$ we have

$g_{D, A} \in \pm 1+v^{-1} \mathbf{Z}\left[v^{-1}\right]$ for one value of $D$, denoted by $D=A^{*}$;

$g_{D, A} \in v^{-1} \mathbf{Z}\left[v^{-1}\right]$ for all $D \neq A^{*}$.

Thus, $\mathbf{t}_{1}$ maps $\mathfrak{m}_{c}$ into itself and we have $\mathbf{t}_{1}(A)=A^{*} \bmod v^{-1} \mathfrak{m}_{c}$. Since $\mathbf{t}_{1}^{2}=$ $z z^{\dagger}: \mathfrak{m}_{c} \rightarrow \mathfrak{m}_{c}$ is the identity map modulo $v^{-1} \mathfrak{m}_{c}$, it follows that

$\left(A^{*}\right)^{*}=1$ for any $A$.

Lemma 12.5. (a) For any $A \leq D$ we have $r_{A, D} \in v^{-l\left(w_{0 *}\right)} \mathbf{Z}[v]$; more precisely, $r_{A, D} \in \pm 1+v^{-l\left(w_{0 *}\right)+1} \mathbf{Z}[v]$ if $D=\kappa_{0}\left(A^{*}\right)$ and $r_{A, D} \in v^{-l\left(w_{0 *}\right)+1} \mathbf{Z}[v]$ if $D \neq \kappa_{0} A^{*}$.

(b) For any $D \leq A$ we have $\tilde{r}_{D, A} \in v^{-b(e)} \mathbf{Z}[v]$; more precisely, $\tilde{r}_{D, A} \in \pm 1+$ $v^{-b(e)+1} \mathbf{Z}[v]$ if $D=\kappa_{0}\left(\rho A^{*}\right)$ and $\tilde{r}_{D, A} \in v^{-b(e)+1} \mathbf{Z}[v]$ if $D \neq \kappa_{0}\left(\rho A^{*}\right)$. 
From 10.6(b) we deduce

(c) $\kappa_{0} \mathbf{t}_{1} \kappa_{0}=\bar{z}^{\dagger} z^{-1} \mathbf{t}_{1}: \mathbf{M}_{c} \rightarrow \mathbf{M}_{c}$.

The bijection $\kappa_{0}: \mathbf{M}_{c} \rightarrow \mathbf{M}_{c}$ extends in an obvious way to bijections $\kappa_{0}^{\prime}: \mathbf{M}_{\leq} \stackrel{\sim}{\rightarrow}$ $\mathbf{M}_{\geq}, \kappa_{0}^{\prime \prime}: \mathbf{M}_{\geq} \stackrel{\sim}{\rightarrow} \mathbf{M}_{\leq}$(inverse to each other).

We write $z^{-1}=\sum_{\tau \in \hat{C}_{\leq}} z_{\tau} \tau$ where $z_{\tau} \in v^{-1} \mathbf{Z}\left[v^{-1}\right]$ for $\tau \in \hat{C}_{\leq}, \tau \neq 1$ and $z_{1}=1$.

We prove (a). In $\mathbf{M}_{\leq}$we have

$$
\begin{aligned}
& \sum_{D^{\prime}} \bar{r}_{A, D^{\prime}} \kappa_{0}\left(D^{\prime}\right)=\kappa_{0}^{\prime \prime} \sum_{D^{\prime}} r_{A, D^{\prime}} D^{\prime}=\kappa_{0}^{\prime \prime} \mathbf{b}^{\prime}(A) \\
& =\kappa_{0}^{\prime \prime}\left((-v)^{-l\left(w_{0 *}\right)} z^{-1} \mathbf{t}_{1}\left(\kappa_{0}(A)\right)\right)=(-v)^{l\left(w_{0 *}\right)}\left(\bar{z}^{\dagger}\right)^{-1} \kappa_{0} \mathbf{t}_{1} \kappa_{0}(A) \\
& =(-v)^{l\left(w_{0 *}\right)} z^{-1} \mathbf{t}_{1}(A)=(-v)^{l\left(w_{0 *}\right)} z^{-1} \sum_{D} g_{D, A} D
\end{aligned}
$$

Hence

$$
\bar{r}_{A, \kappa_{0} D}=\sum_{\tau}(-v)^{l\left(w_{0 *}\right)} z_{\tau} \epsilon_{\tau} g_{\tau^{-1} * D, A} .
$$

By 12.4, the right hand side is in $v^{l\left(w_{0 *}\right)} \mathbf{Z}\left[v^{-1}\right]$. The first assertion of (a) follows. The same argument gives the second assertion of (a).

We prove (b). In $\mathbf{M}_{\geq}$we have

$$
\begin{aligned}
& \sum_{D^{\prime}} \overline{\tilde{r}}_{D^{\prime}, A} \kappa_{0}\left(D^{\prime}\right)=\kappa_{0}^{\prime} \sum_{D^{\prime}} \tilde{r}_{D^{\prime}, A} D^{\prime}=\kappa_{0}^{\prime} \tilde{\mathbf{b}}^{\prime}(A) \\
& =\kappa_{0}^{\prime}\left((-1)^{l\left(w_{0 *}\right)}(-v)^{-b(e)} \rho\left(z^{\dagger}\right)^{-1} \mathbf{t}_{1}\left(\kappa_{0}(A)\right)\right) \\
& =(-1)^{l\left(w_{0 *}\right)}(-v)^{b(e)} \rho^{-1} \bar{z}^{-1} \kappa_{0} \mathbf{t}_{1} \kappa_{0}(A)=(-1)^{l\left(w_{0 *}\right)}(-v)^{b(e)} \rho\left(z^{\dagger}\right)^{-1} \mathbf{t}_{1}(A) .
\end{aligned}
$$

Hence

$$
\overline{\tilde{r}}_{\kappa_{0} D, A}=\sum_{\tau}(-1)^{l\left(w_{0 *}\right)}(-v)^{b(e)} z_{\tau} \epsilon_{\tau} \epsilon_{\rho} g_{\left(\rho^{-1} \tau\right) * D, A} .
$$

By 12.4, the right hand side is in $v^{b(e)} \mathbf{Z}\left[v^{-1}\right]$. The first assertion of (b) follows. The same argument gives the second assertion of (b). The lemma is proved.

Proposition 12.6. (a) For $B \leq A$ we have $\pi_{B, A} \in v^{-l\left(w_{0 *}\right)} \mathbf{Z}[v]$. More precisely, $\pi_{B, A} \in v^{-l\left(w_{0 *}\right)+1} \mathbf{Z}[v]$ if $D \neq \kappa_{0}\left(A^{*}\right)$ and $\pi_{B, A} \in \pm v^{-l\left(w_{0 *}\right)}+v^{-l\left(w_{0 *}\right)+1} \mathbf{Z}[v]$ if $D=\kappa_{0}\left(\rho A^{*}\right)$.

(b) For $A \leq B$ we have $\tilde{\pi}_{A, B} \in v^{-b(e)} \mathbf{Z}[v]$. More precisely, $\tilde{\pi}_{B, A} \in v^{-b(e)+1} \mathbf{Z}[v]$ if $D \neq \kappa_{0}\left(\rho A^{*}\right)$ and $\tilde{\pi}_{A, B} \in \pm v^{-b(e)}+v^{-b(e)+1} \mathbf{Z}[v]$ if $D=\kappa_{0}\left(\rho A^{*}\right)$.

We prove (a). For $B \leq D$ we have as in the proof of 11.5 :

(c) $\pi_{B, D}=\sum_{A ; B \leq A \leq D} \overline{\pi_{B, A}} r_{A, D}$.

For any $A$ in the sum we have $\pi_{B, A} \in \mathbf{Z}\left[v^{-1}\right]$ hence $\overline{\pi_{B, A}} \in \mathbf{Z}[v]$ and $r_{A, D} \in$ $v^{-l\left(w_{0 *}\right)} \mathbf{Z}[v]$ (by $12.5(\mathrm{a})$ ). Hence the right hand side of (c) is in $v^{-l\left(w_{0 *}\right)} \mathbf{Z}[v]$. The first assertion of (a) is proved. The same argument gives the second assertion of (a).

We prove (b). For $D \leq B$ we have as in the proof of 11.5:

(d) $\tilde{\pi}_{D, B}=\sum_{A ; D \leq A \leq B} \tilde{r}_{D, A} \overline{\tilde{\pi}_{A, B}}$.

For any $A$ in the sum we have $\pi_{A, B} \in \mathbf{Z}\left[v^{-1}\right]$ hence $\overline{\tilde{\pi}_{A, B}} \in \mathbf{Z}[v]$ and $\tilde{r}_{D, A} \in$ $v^{-b(e)} \mathbf{Z}[v]$ (by 12.5(b)). Hence the right hand side of (d) is in $v^{-b(e)} \mathbf{Z}[v]$. The first assertion of (b) is proved. The same argument gives the second assertion of (b). The proposition is proved. 
12.7. We conjecture that for $B \in X_{\Xi}$, we have $B_{\leq} \in \mathbf{M}_{c}, B_{\geq} \in \mathbf{M}_{c}$. Note that 12.6 provides some support for this conjecture by showing that there are only finitely many powers of $v$ that can appear with a non-zero coefficient in some $\pi_{B, A}$ or in some $\tilde{\pi}_{B, A}$ (with $A$ variable). In the remainder of this section we develop a method which shows at least that if all of $B_{\leq}$are in $\mathbf{M}_{c}$, then all of $B_{\geq}$are in $\mathbf{M}_{c}$ (and conversely).

12.8. Let $R_{H, \text { int }}=\mathbf{Z}[\hat{C}]\left(\left(v^{-1}\right)\right)$ be the ring of power series in $v^{-1}$ with coefficients in the group ring of $\hat{C}$ over $\mathbf{Z}$. Since $R_{H}=\mathbf{Z}[\hat{C}]\left[v, v^{-1}\right]$, we can regard $R_{H}$ naturally as a subring of $R_{H, \text { int }}$. By 8.15(b), the elements $z, z^{\dagger}$ of $R_{H}$ are invertible in $R_{H, \text { int }}$.

Let $\mathbf{M}_{\text {int }}$ be the subset of $\mathbf{M}$ consisting of the formal sums $m=\sum_{A \in X_{\Xi}} m_{A} A$ (with $m_{A} \in \mathcal{A}$ ) such that

(a) there exists $n_{0} \in \mathbf{Z}$ such that $v^{n_{0}} m_{A} \in \mathbf{Z}\left[v^{-1}\right]$ for all $A \in X_{\Xi}$;

(b) for any $n \in \mathbf{Z}$, there are only finitely many $A \in X_{\Xi}$ such that $v^{n}$ appears with non-zero coefficient in $m_{A}$.

Note that $\mathbf{M}_{c}$ is naturally contained in $\mathbf{M}_{i n t}$ and that the $R_{H}$-module structure on $\mathbf{M}_{c}$ extends naturally to an $R_{H, \text { int }}$-module structure on $\mathbf{M}_{\text {int }}$.

Proposition 12.9. The following six statements are equivalent.

(1) $B_{\geq} \in \mathbf{M}_{\text {int }}$ for all $B \in X_{\Xi}$;

(2) $B \leq \in \mathbf{M}_{\text {int }}$ for all $B \in X_{\Xi}$;

(3) $\hat{B}_{\geq} \in \mathbf{M}_{\text {int }}$ for all $B \in X_{\Xi}$;

(4) $\check{B}_{\leq} \in \mathbf{M}_{\text {int }}$ for all $B \in X_{\Xi}$;

(5) $B \geq \in \mathbf{M}_{c}$ for all $B \in X_{\Xi}$;

(6) $B_{\leq} \in \mathbf{M}_{c}$ for all $B \in X_{\Xi}$.

Since $B_{\leq}=z \check{B_{\leq}}$and $z$ is invertible in $R_{H, \text { int }}$, we see that (2),(4) are equivalent. Since $B_{\geq}=z^{\dagger} \hat{B_{\geq}}$and $z^{\dagger}$ is invertible in $R_{H, i n t}$, we see that (1),(3) are equivalent. We show that (1),(5) are equivalent. Trivially, if (5) holds, then (1) holds. Assume now that (1) holds. Let $B \in X_{\Xi}$. By (1) there are only finitely many $A$ such that $v^{n}$ with $n \in\left[-l\left(w_{0 *}\right), 0\right]$ appears with non-zero coefficient in $\pi_{B, A}$. By 12.6(a), if $n \notin\left[-l\left(w_{0 *}\right), 0\right]$, then $v^{n}$ appears with coefficient in $\pi_{B, A}$ for any $A$. It follows that $B \geq \in \mathbf{M}_{c}$ and (5) holds.

Similarly, (2),(6) are equivalent. Thus, (1),(3),(5) are equivalent and (2),(4),(6) are equivalent.

We show that (6) implies (3). Since (6) is assumed to hold,

(a) for any $B$, the set $\left\{A \mid \tilde{\pi}_{A, B} \neq 0\right\}$ is finite.

We show that

(b) for any $A$, the set $\left\{B \mid \tilde{\pi}_{A, B} \neq 0\right\}$ is finite.

We can find a finite subset $B_{1}, B_{2}, \ldots, B_{m}$ of $X_{\Xi}$ so that any $B \in X_{\Xi}$ is of the form $\tau * B_{k}$ for a unique $k \in[1, m]$ and a unique $\tau \in \hat{C}$. To show (b) we must show that $\left\{(k, \tau) \mid \tilde{\pi}_{A, \tau * B_{k}} \neq 0\right\}$ is finite. From the definitions we have $\tilde{\pi}_{A, \tau * B_{k}}=$ $\tilde{\pi}_{\tau^{-1} * A, B_{k}}$. Thus we must show that $Z=\left\{(k, \tau) \mid \tilde{\pi}_{\tau^{-1} * A, B_{k}} \neq 0\right\}$ is finite. If $(k, \tau) \in Z$, then using (a) for $B=B_{k}$, we see that $\tau^{-1} * A$ must belong to a fixed finite set. Hence $\tau$ must belong to a fixed finite set. Hence $Z$ is finite and (b) is proved.

Let $D \in X_{\Xi}$ and let any $n \in \mathbf{Z}$. Let $Y_{D, n}$ be the set of all $A \in X_{\Xi}$ such that $v^{n}$ appears with non-zero coefficient in $\hat{\pi}_{D, A}$. If $n>0$, then $Y_{D, n}=\emptyset$. If $n=0$, then $Y_{D, n}=\{D\}$. Assume now that $n<0$. 
From $11.5(\mathrm{a})$ we see that

$$
\hat{\pi}_{D, A}=\sum(-1)^{r} \tilde{\pi}_{A_{0}, A_{1}} \tilde{\pi}_{A_{1}, A_{2}} \ldots \tilde{\pi}_{A_{r-1}, A_{r}}
$$

where the sum is taken over all sequences $A_{0}, A_{1}, \ldots, A_{r}$ in $X_{\Xi}$ such that $A=A_{0}<$ $A_{1}<\cdots<A_{r}=D$. Note that each $p_{A_{j}, A_{j+1}}$ is in $v^{-1} \mathbf{Z}\left[v^{-1}\right]$. Hence, if $A \in Y_{D, n}$, then in the sum above we may restrict $r$ to be in $[0,-n]$. By (b) there are only finitely many sequences $A_{0}, A_{1}, \ldots, A_{r}$ in $X_{\Xi}$ such that $r \in[0,-n], A_{r}=D$ and

$$
\tilde{\pi}_{A_{0}, A_{1}} \tilde{\pi}_{A_{1}, A_{2}} \ldots \tilde{\pi}_{A_{r-1}, A_{r}} \neq 0 \text {. }
$$

Since $A=A_{0}$ for such a sequence we see that $A$ is in a fixed finite set. Thus, $Y_{D, n}$ is finite for any $D, n$. We see that $\hat{D}_{\geq} \in \mathbf{M}_{\text {int }}$. We have thus proved that (6) implies (3).

An entirely similar proof shows that (5) implies (4). The proposition is proved.

\section{EXAMPle OF AN ELEMENT OF $\mathbf{B}_{\mathcal{B}_{e}}^{ \pm}$}

13.1. We return to the general setup of 1.8. We consider the following conditions on $(e, h, f)$ :

(a) there exists a parabolic subalgebra $\mathfrak{q}^{\prime}$ of $\mathfrak{g}$ such that $e$ is in the Richardson orbit in $\mathfrak{n}_{\mathfrak{q}^{\prime}}$;

(b) $e$ is regular in a Levi subalgebra of some parabolic subalgebra of $\mathfrak{g}$.

For example, (a),(b) certainly hold if $G$ is of type $A$.

In the remainder of this section we will assume that (a),(b) are satisfied. We choose a parabolic subalgebra $\mathfrak{p}$ as in (b) and we define $K^{!}, W^{!}, W_{!}$in terms of $\mathfrak{q}^{\prime}$ in the same way as $K, W^{*}, W_{*}$ were defined in terms of $\mathfrak{p}$ in $6.1,6.14$.

13.2. Let $Y^{\prime}$ be an irreducible component of $\mathcal{B}_{e}$. Since $\mathfrak{Z}$ is partitioned into locally closed subvarieties $\mathfrak{Z}_{w}$ which are irreducible of dimension $b(e)$ and $Y^{\prime}$ is a projective, irreducible subvariety of $\mathfrak{Z}$ of dimension $b(e)$, there exists a unique $w \in W_{*}$ such that $\mathfrak{Z}_{w}$ is an open dense subset of $Y^{\prime}$.

The map $Y^{\prime} \mapsto w$ is an injective map from the set of irreducible components of $\mathcal{B}_{e}$ into $W_{*}$.

13.3. Let $Y=\left\{\mathfrak{b} \in \mathcal{B} \mid \mathfrak{b} \subset \mathfrak{q}^{\prime}\right\}$. From 13.1(a) it follows that $Y$ is an irreducible component of $\mathcal{B}_{e}$. We can find an $H$-equivariant line bundle $\mathcal{L}$ on $Y$ such that $\underline{\mathcal{L}} \otimes \underline{\mathcal{L}}$ is isomorphic to $f \Omega$ where $\Omega$ is the canonical line bundle of $Y$ and $f \in \hat{H}$. Moreover, $\underline{\mathcal{L}}$ is unique up to multiplication by an element in $\hat{H}$.

By 13.2 , there is a unique $w \in W_{*}$ such that $\mathfrak{Z}_{w}$ is an open dense subset of $Y$.

Lemma 13.4. For any $i \in K^{!}$we have $\tilde{T}_{\sigma_{i}} \underline{\mathcal{L}}=v \underline{\mathcal{L}}$.

The proof is along the lines of the proof of [L6, 3.8].

In the remainder of this section we assume that $G$ is adjoint.

Lemma 13.5. Let $w \in W_{*}$ be as in 13.3. We can normalize $\underline{\mathcal{L}}$ uniquely so that $\Phi: \mathfrak{E}_{e} \stackrel{\sim}{\rightarrow}\left(\mathfrak{m}_{c}\right)_{\mathfrak{R}}$ carries $\underline{\mathcal{L}}$ to $(-1)^{l(w)+b(e) w^{-1}} A_{0}^{+}$plus an $R_{H}$-linear combination of elements ${ }^{w^{\prime-1}} A_{0}^{+}$with $w^{\prime} \in W_{*}, w^{\prime}<w$.

The image of $\mathcal{L}$ under $K_{H}\left(\mathfrak{Z}_{\leq w}\right) \rightarrow K_{H}\left(\mathfrak{Z}_{w}\right)$ (inverse image under the open imbedding $\left.\mathfrak{Z}_{w} \subset \mathfrak{Z}_{\leq w}\right)$ is just the restriction of the line bunde $\underline{\mathcal{L}}$ to $\mathfrak{Z}_{w}$. Using 6.3, we see that any $H$-equivariant line bundle on $\mathfrak{Z}_{w}$ is of the form $f \mathfrak{O}_{\mathfrak{Z}_{w}}$ for some $f \in \hat{H}$. Replacing if necessary $\underline{\mathcal{L}}$ by $f^{-1} \underline{\mathcal{L}}$, we may assume that the image of 
$\underline{\mathcal{L}}$ under $K_{H}\left(\mathfrak{Z}_{\leq w}\right) \rightarrow K_{H}\left(\mathfrak{Z}_{w}\right)$ is $v^{b(e)} v^{-l(w)} \rho \mathfrak{O}_{\mathfrak{Z}_{w}}$. By the proof of 8.9, we have $\tilde{T}_{w} \mathfrak{O}_{\mathfrak{Z}_{1}} \in K_{H}\left(\overline{\mathfrak{Z}}_{\leq w}\right)$ and the image of this element under $K_{H}\left(\mathfrak{Z}_{\leq w}\right) \rightarrow K_{H}\left(\mathfrak{Z}_{w}\right)$ is $(-v)^{-l(w)} \mathfrak{O}_{\mathfrak{Z}_{w}}$. Thus, $\underline{\mathcal{L}}-(-1)^{l(w)} v^{b(e)} \rho \tilde{T}_{w} \mathfrak{O}_{\mathfrak{Z}_{1}}$ is in the kernel of $K_{H}\left(\mathfrak{Z}_{\leq w}\right) \rightarrow$ $K_{H}\left(\mathfrak{Z}_{w}\right)$ hence it belongs to $K_{H}\left(\mathfrak{Z}_{<w}\right)$; it is then an $R_{H}$-linear combination of elements of the form $\tilde{T}_{w^{\prime}} \mathfrak{O}_{\mathfrak{Z}_{1}}$ with $w^{\prime} \in W_{*}, w^{\prime}<w$ (again by the arguments in 8.9). Now $\tilde{T}_{w} \mathfrak{O}_{\mathfrak{Z}_{1}}$ is equal to $\tilde{T}_{w^{-1}}^{-1} \mathfrak{O}_{\mathfrak{Z}_{1}}$ plus an $\mathcal{A}$-linear combination of elements $\tilde{T}_{w^{\prime-1}}^{-1} \mathfrak{O}_{\mathfrak{Z}_{1}}$ with $w^{\prime} \in W_{*}, w^{\prime}<w$. It follows that $\underline{\mathcal{L}}$ equals $(-1)^{l(w)} v^{b(e)} \rho \tilde{T}_{w^{-1}}^{-1} \mathfrak{O}_{\mathfrak{Z}_{1}}$ plus an $R_{H^{-}}$-linear combination of elements $v^{b(e)} \rho \tilde{T}_{w^{\prime-1}}^{-1} \mathfrak{O}_{\mathfrak{Z}_{1}}$ with $w^{\prime} \in W_{*}, w^{\prime}<w$. Now the result follows from 10.2 .

Lemma 13.6. Let $m^{\prime}=\sum_{w^{\prime} \in W_{*}} g_{w^{\prime}}\left(w^{w^{-1}} A_{0}^{+}\right) \in \mathbf{M}_{c}$ where $g_{w^{\prime}} \in R_{H}$. Let $i \in I$. The following two conditions are equivalent:

(a) $\tilde{T}_{\sigma_{i}} m=v m$;

(b) $g_{\sigma_{i} w^{\prime}}=v g_{w^{\prime}}$ for any $w \in W_{*}$ such that $l\left(\sigma_{i} w\right)=l(w)-1$ and $g_{w^{\prime}}=0$ for any $w^{\prime} \in W_{*}$ such that $l\left(\sigma_{i} w^{\prime}\right)=l\left(w^{\prime}\right)+1, \sigma_{i} w^{\prime} \notin W_{*}$.

The proof is along the same lines as that of 8.14.

13.7. We consider the following property of $K, K^{!}$:

(a) $\operatorname{dim}\left\{m^{\prime} \in\left(\mathbf{M}_{c}\right)_{\mathfrak{R}} \mid \tilde{T}_{\sigma_{i}} m^{\prime}=v m^{\prime} \forall i \in K^{!}\right\}=1$.

One can show that this property is satisfied if $G=P G L_{n}$.

In the remainder of this section we assume that (a) is satisfied for our $(e, h, f)$.

13.8. Let $w \in W_{*}$ be as in 13.3. Let $m=(-1)^{l(w)+b(e)} \Phi(\underline{\mathcal{L}}) \in \mathbf{M}_{c}$. (We assume that $\mathcal{L}$ is normalized as in 13.5.) By 13.4, 13.5, we have

(a) $m=w^{-1} A_{0}^{+}+\sum_{w^{\prime} \in W_{*}, w^{\prime}<w} g_{w^{\prime}}\left(w^{w^{\prime-1}} A_{0}^{+}\right)$, where $g_{w^{\prime}} \in R_{H}$,

(b) $\tilde{T}_{\sigma_{i}} m=v m$ for any $i \in K^{!}$.

Lemma 13.9. (a) $w$ has maximal length in $W^{!} w$.

(b) $W^{!} w \subset W_{*}$.

We prove (a). We write $m=\sum_{w^{\prime} \in W_{*}} g_{w^{\prime}}\left(w^{\prime-1} A_{0}^{+}\right)$as in 13.6. It suffices to show that $l\left(\sigma_{i} w\right)<l(w)$ for any $i \in K^{!}$. Assume that $i \in K^{!}$and $l\left(\sigma_{i} w\right)=l(w)+1$. Since $g_{w} \neq 0$, we see from 13.6(b) that $\sigma_{i} w \in W_{*}$ and that $g_{\sigma_{i} w}=v^{-1} g_{w} \neq 0$. This contradicts 13.8(a).

We prove (b). We show that $y \in W_{*}$ for $y \in W^{!} w$ by descending induction on $l(y)$. Our claim is true in the case where $y$ has maximal length in $W^{!} w$ by (a). Assume now that $y \in W_{*}, l(y)<l(w)$. We can find $i \in K^{!}$such that $l\left(\sigma_{i} y\right)=$ $l(y)+1$. By the induction hypothesis we have $\sigma_{i} y \in W_{*}$. This implies $y \in W_{*}$. (In general, if $y^{\prime} \in W_{*}$ and $\sigma_{i} y^{\prime}<y^{\prime}$ then $\sigma_{i} y^{\prime} \in W_{*}$.) The lemma is proved.

13.10. Using 13.7(a), we see that the vector $m$ in 13.8 is proportional to the vector $\sum_{t \in W^{!}} v^{l(t)}\left({ }^{(t w)^{-1}} A_{0}^{+}\right)$, which belongs to the space in 13.7(a), by 13.9(b) and 13.6. Since ${ }^{w^{-1}} A_{0}^{+}$appears with coefficient 1 in both vectors, we see that

(a) $m=\sum_{t \in W^{!}} v^{l(t)}\left({ }^{(t w)^{-1}} A_{0}^{+}\right)$.

13.11. From 13.7(a) we see also that

(a) $w$ is the unique element of $W_{*}$ such that $w$ has maximal length in $W^{!} w$.

Indeed, if $w^{\prime} \in W_{*}$ has maximal length in $W^{!} w^{\prime}$, then $\sum_{t \in W^{!}} v^{l(t)}\left({ }^{\left(t w^{\prime}\right)^{-1}} A_{0}^{+}\right)$ would again be a vector in the space in 13.7(a), hence it would be proportional to $m$, which forces $w=w^{\prime}$. 
Lemma 13.12. $\tilde{\mathbf{b}}(m)=v^{-2 b(e)} m$.

From the $\mathcal{H}$-semilinearity property of $\tilde{\mathbf{b}}:\left(\mathbf{M}_{c}\right)_{\Re} \rightarrow\left(\mathbf{M}_{c}\right)_{\mathfrak{R}}$ we see easily that $\tilde{\mathbf{b}}$ maps the space in 13.7 (a) into itself. In particular, we have $\tilde{\mathbf{b}}(m)=f m$ for some $f \in \mathfrak{R}$. Since $\tilde{\mathbf{b}}$ maps $\mathbf{M}_{c}$ into $\left(z^{\dagger}\right)^{-1} \mathbf{M}_{c}$, we must have $f \in\left(z^{\dagger}\right)^{-1} R_{H}$. Then the equality $\tilde{\mathbf{b}}(m)=f m$ implies the equality $\tilde{\mathbf{b}}^{\prime}(m)=f m$ in $\mathbf{M}_{\leq}$. Let $w_{0}^{!}$be the longest element of $W^{!}$. Since $l\left(w_{0}^{!}\right)=b(e)$, we have $m=v^{b(e)}\left({ }^{\left(w_{0}^{\leftarrow} w\right)^{-1}} A_{0}^{+}\right)$plus an $\mathcal{A}$-linear combination of alcoves $A^{\prime}<\left(w_{0}^{!} w\right)^{-1} A_{0}^{+}$.

Using 9.16(b), we deduce that $\tilde{\mathbf{b}}^{\prime}(m)=v^{-b(e)}\left({\left(w_{0}^{!} w\right)^{-1}}^{+} A_{0}^{+}\right)$plus a formal $\mathcal{A}$-linear combination of alcoves $A^{\prime}<{ }^{\left(w_{0}^{\prime} w\right)^{-1}} A_{0}^{+}$.

Using now $\tilde{\mathbf{b}}^{\prime}(m)=f m$ and comparing the coefficients of $\left(w_{0}^{!} w\right)^{-1} A_{0}^{+}$, we see that $v^{-b(e)}=f v^{b(e)}$. The lemma follows.

Theorem 13.13. (a) We have $v^{-b(e)} m=B_{\leq}$where $B=\left(w_{0}^{!} w\right)^{-1} A_{0}^{+}$.

(b) We have $v^{-b(e)} \underline{\mathcal{L}} \in \mathbf{B}_{\mathcal{B}_{e}}^{ \pm}$.

From 13.10(a) we have

$$
v^{-b(e)} m=\sum_{t \in W^{!}} v^{l(t)-b(e)}\left({ }^{(t w)^{-1}} A_{0}^{+}\right) \in B+v^{-1} \mathfrak{m}_{c} ;
$$

from 13.12, we have $\tilde{\mathbf{b}}\left(v^{-b(e)} m\right)=v^{-b(e)} m$. This proves (a). Now (b) follows from (a), using 10.7(c). The theorem is proved.

14. A case Where the $\left(\mathcal{H}, R_{H}\right)$-module $K_{H}\left(\mathcal{B}_{e}\right)$ Is CyClic

14.1. In this section we assume that $(e, h, f)$ satisfies 13.1(a),(b). In fact we shall assume that, in addition, the following strengthened form of 13.1(a) holds:

(a) there exists a homomorphism $\iota: \mathbf{C}^{*} \rightarrow G$ with corresponding weight decomposition $\mathfrak{g}=\bigoplus_{n \in 2 \mathbf{Z}} \mathfrak{g}^{n}$ such that, if we set $\mathfrak{q}^{\prime}=\bigoplus_{n \geq 0} \mathfrak{g}^{n}$ (with corresponding subgroup $\left.Q^{\prime}\right), \mathfrak{n}^{\prime}=\bigoplus_{n>0} \mathfrak{g}^{n}$, then $e \in \mathfrak{g}^{2}, h \in \mathfrak{g}^{0}, f \in \mathfrak{g}^{-2}$ and $e$ is in the unique open $Q^{\prime}$-orbit in $\mathfrak{n}^{\prime}$.

It is likely that this automatically holds in the presence of $13.1(\mathrm{a}),(\mathrm{b})$. It certainly holds if $G=S L_{N}$. (See 14.12.)

14.2. Let $\iota, \mathfrak{q}^{\prime}, \mathfrak{n}^{\prime}, \mathfrak{l}^{\prime}=\mathfrak{g}^{0}$ be as in $14.1(\mathrm{a})$. Let

$$
\begin{gathered}
\tilde{Z}(e, h, f)=\left\{(g, \lambda) \in G \times \mathbf{C}^{*} \mid \operatorname{Ad}(g) e=\lambda^{2} e, \operatorname{Ad}(g) h=h, \operatorname{Ad}(g) f=\lambda^{-2} f\right\}, \\
Z(e, h, f)=\{g \in G \mid \operatorname{Ad}(g) e=e, \operatorname{Ad}(g) h=h, \operatorname{Ad}(g) f=f\} .
\end{gathered}
$$

We have an isomorphism

(a) $Z(e, h, f)^{0} \times \mathbf{C}^{*} \stackrel{\sim}{\rightarrow} \tilde{Z}(e, h, f)^{0}$

given by $(g, \lambda) \mapsto(g \phi(\lambda), \lambda)$.

Note that $T_{1}=\left\{(\iota(\lambda), \lambda) \mid \lambda \in \mathbf{C}^{*}\right\} \subset \tilde{Z}(e, h, f)^{0}$. Also $T_{2}=\left\{(\phi(\lambda), \lambda) \mid \lambda \in \mathbf{C}^{*}\right\}$ is in the centre of $\tilde{Z}(e, h, f)^{0}$. Under (a), $T_{2}$ corresponds to $1 \times \mathbf{C}^{*}$ and $T_{1}$ corresponds to a one-dimensional torus $T_{1}^{\prime}$ in $Z(e, h, f)^{0} \times \mathbf{C}^{*}$. Now $T_{1}^{\prime}$ is contained in some maximal torus of $Z(e, h, f)^{0} \times \mathbf{C}^{*}$ (necessarily of form $C \times \mathbf{C}^{*}$ where $C$ is a maximal torus of $\left.Z(e, h, f)^{0}\right)$. We shall assume that $C$ in 1.8 is the one just considered. Then $T_{1} \subset C T_{2}$, hence

(b) $\iota\left(\mathbf{C}^{*}\right) \subset C \phi\left(\mathbf{C}^{*}\right)$. 
Now let $\mathfrak{l}$ be the centralizer of $C$ in $\mathfrak{g}$ and let $\mathfrak{p}$ be any parabolic subalgebra of $\mathfrak{g}$ with Levi $\mathfrak{l}$. We have $e, h, f \in \mathfrak{l}$. Condition 13.1(b) implies that $e$ is regular in $\mathfrak{l}$. Thus, $\mathfrak{p}, \mathfrak{l}$ are as in 8.1.

14.3. As in 13.1 , let $K^{!}, W^{!}, W_{!}, \leq$be defined in terms of $\mathfrak{q}^{\prime}$ in the same way as $K, W^{*}, W_{*}, \leq$ were defined in terms of $\mathfrak{p}$ in $6.1,6.14$. We define a partition

$$
\mathcal{B}_{e}=\bigsqcup_{w \in W !} \mathcal{B}_{e, w}
$$

where $\mathcal{B}_{e, w}$ is the set of all $\mathfrak{b} \in \mathcal{B}_{e}$ such that, for some $\mathfrak{b}^{\prime} \in \mathcal{B}, \mathfrak{b}^{\prime} \subset \mathfrak{q}^{\prime}$, the relative position of $\mathfrak{b}, \mathfrak{b}^{\prime}$ is $w$.

Since $e$ is a Richardson element of $\mathfrak{n}^{\prime}$ (see 13.1(a)), we can apply [DLP, 3.2, 3.3] and we see that

(a) each $\mathcal{B}_{e, w}$ is smooth of pure dimension $b(e)$.

Let $\mathcal{B}_{e, \leq w}=\bigcup_{w_{1} \in W_{!} ; w_{1} \leq w} \mathcal{B}_{e, w_{1}}, \mathcal{B}_{e,<w}=\mathcal{B}_{e, \leq w}-\mathcal{B}_{e, w}$.

Again, since $e$ is a Richardson element of $\mathfrak{n}^{\prime}, H$ stabilizes $\mathfrak{q}^{\prime}$; it follows that $\mathcal{B}_{e, w}, \mathcal{B}_{e, \leq w}, \mathcal{B}_{e,<w}$ are $H$-stable subvarieties of $\mathcal{B}$.

14.4. Let $w \in W_{!}, i \in I$ be such that $\sigma_{i} w=w^{\prime}<w$. Then $w^{\prime} \in W_{\text {! }}$ and we can define $\zeta: \mathcal{B}_{e, w} \rightarrow \mathcal{B}_{e, w^{\prime}}$ as follows.

Let $\mathfrak{b} \in \mathcal{B}_{e, w}$. There is a unique $\mathfrak{b}^{\prime} \in \mathcal{B}$ such that $\mathfrak{b}, \mathfrak{b}^{\prime}$ are in relative position $\sigma_{i}$ and $\mathfrak{b}^{\prime}, \mathfrak{b}^{\prime \prime}$ are in relative position $w^{\prime}$, for some $\mathfrak{b}^{\prime \prime} \in \mathcal{B}, \mathfrak{b}^{\prime \prime} \subset \mathfrak{q}^{\prime}$. Since $e \in \mathfrak{q}^{\prime}$, $\exp (e) \mathfrak{b}^{\prime}$ satisfies the defining properties of $\mathfrak{b}^{\prime}$, hence it is equal to $\mathfrak{b}^{\prime}$. Thus, $e \in \mathfrak{b}^{\prime}$, hence $\mathfrak{b}^{\prime} \in \mathcal{B}_{e, w^{\prime}}$. This defines $\zeta$.

Since $\mathfrak{b}, \mathfrak{b}^{\prime}$ both contain $e$ and are in relative position $\sigma_{i}$, it follows that any point on the $i$-line in $\mathcal{B}$ containing $\mathfrak{b}$ must contain $e$. Hence that line (minus $\mathfrak{b}^{\prime}$ ) is contained in $\mathcal{B}_{e, w}$. Thus, $\zeta$ is a line bundle over its image, a closed smooth submanifold of $\mathcal{B}_{e, w^{\prime}}$ of pure codimension 1. Since $\mathcal{B}_{e, w}, \mathcal{B}_{e, w^{\prime}}$ are smooth (14.3(a)), the map $\zeta^{*}: K_{H}\left(\mathcal{B}_{e, w^{\prime}}\right) \rightarrow K_{H}\left(\mathcal{B}_{e, w}\right)$ is well defined.

Lemma 14.5. $\zeta^{*}$ is surjective.

By an argument like the one in 1.13(a),(b), we see that $\mathcal{B}_{e, w}, \mathcal{B}_{e, w^{\prime}}$ are vector bundles over $\mathcal{B}_{e, w}^{\iota\left(\mathbf{C}^{*}\right)}, \mathcal{B}_{e, w^{\prime}}^{\left(\mathbf{C}^{*}\right)}$. We are reduced to showing that

$$
j^{*}: K_{H}\left(\mathcal{B}_{e, w^{\prime}}^{\iota\left(\mathbf{C}^{*}\right)}\right) \rightarrow K_{H}\left(\mathcal{B}_{e, w}^{\iota\left(\mathbf{C}^{*}\right)}\right)
$$

is surjective, where $j: \mathcal{B}_{e, w}^{\iota\left(\mathbf{C}^{*}\right)} \rightarrow \mathcal{B}_{e, w^{\prime}}^{\iota\left(\mathbf{C}^{*}\right)}$ is a natural imbedding (of smooth projective varieties) induced by $\zeta$. Note that the fixed point sets of $\iota\left(\mathbf{C}^{*}\right)$ are $H$-stable, by 14.2(b).

Now $j^{*}, j_{*}$ are adjoint with respect to the bilinear forms 1.5(a) for these two varieties, and these bilinear forms are perfect, by 1.7 (c) (since the $H$-action on both varieties has only finitely many fixed points, by 13.1(b)). We are therefore reduced to showing that $j_{*}: K_{H}\left(\mathcal{B}_{e, w}^{\iota\left(\mathbf{C}^{*}\right)}\right) \rightarrow K_{H}\left(\mathcal{B}_{e, w^{\prime}}^{\iota\left(\mathbf{C}^{*}\right)}\right)$ is injective. But after tensoring with $\mathfrak{R}$ over $R_{H}$, it is certainly injective since for the fixed point sets of $H$ we just have the inclusion of one finite set in another finite set. Since our $K_{H}$ groups are free over $R_{H}$, the map is also injective before tensoring. The lemma is proved. 
14.6. In the setup of $14.4, \mathcal{B}_{e, \leq w}$ is an $i$-saturated subvariety of $\mathcal{B}_{e}$. Hence $r_{i}$ : $K_{H}\left(\mathcal{B}_{e, \leq w}\right) \rightarrow K_{H}\left(\mathcal{B}_{e, \leq w}\right)$ is defined. As in 7.5 , there is a unique $R_{H}$-linear map

$$
f: K_{H}\left(\mathcal{B}_{e, w^{\prime}}\right) \rightarrow K_{H}\left(\mathcal{B}_{e, w}\right)
$$

such that the composition

$$
K_{H}\left(\mathcal{B}_{e, \leq w^{\prime}}\right) \rightarrow K_{H}\left(\mathcal{B}_{e, w^{\prime}}\right) \stackrel{f}{\rightarrow} K_{H}\left(\mathcal{B}_{e, w}\right)
$$

(where the first map is inverse image under $\mathcal{B}_{e, w^{\prime}} \subset \mathcal{B}_{e, \leq w^{\prime}}$ ) is equal to the composition

$$
K_{H}\left(\mathcal{B}_{e, \leq w^{\prime}}\right) \rightarrow K_{H}\left(\mathcal{B}_{e, \leq w}\right) \stackrel{r_{i}}{\longrightarrow} K_{H}\left(\mathcal{B}_{e, \leq w}\right) \rightarrow K_{H}\left(\mathcal{B}_{e, w}\right)
$$

(where the first map is direct image under $\mathcal{B}_{e, \leq w^{\prime}} \subset \mathcal{B}_{e, \leq w}$ and the third map is inverse image under $\mathcal{B}_{e, w} \subset \mathcal{B}_{e, \leq w}$ ).

Lemma 14.7. $f=v^{-1} \zeta^{*}$.

The proof is similar to that of 7.6(a). It is based on the fact that the intersection in $\Lambda \times \Lambda$ (smooth of dimension $4 \nu$ ) of the subvariety $\bar{Z}_{i}$ (smooth of dimension $2 \nu$ ) with the subvariety $\Lambda \times \mathcal{B}_{e, w}$ (smooth of dimension $2 \nu+b(e)$, by $14.3(\mathrm{a})$ ) is transversal: it is $\mathcal{B}_{e, w^{\prime}}$ (smooth of dimension $b(e)$, by 14.3(a)). The lemma follows.

14.8. We regard $K_{H}\left(\mathcal{B}_{e, 1}\right)$ as a subspace of $K_{H}\left(\mathcal{B}_{e}\right)$ via direct image under $\mathcal{B}_{e, 1} \subset$ $\mathcal{B}_{e}$

Lemma 14.9. The $\mathcal{H}$-module $K_{H}\left(\mathcal{B}_{e}\right)$ is generated by $K_{H}\left(\mathcal{B}_{e, 1}\right)$.

The proof is entirely analogous to the proof of surjectivity of the map $7.10(\mathrm{~d})$, given in 7.11; instead of using 7.6, we use 14.5, 14.7.

In 14.10, 14.11, we assume that $G$ is simply connected.

14.10. We have $\mathcal{B}_{e, 1}=Y$ (see 13.3). Note that the subspace $K_{H}\left(\mathcal{B}_{e, 1}\right)$ of $K_{H}\left(\mathcal{B}_{e}\right)$ is stable under the operators $\theta_{x}: K_{H}\left(\mathcal{B}_{e}\right) \rightarrow K_{H}\left(\mathcal{B}_{e}\right)$ with $x \in \mathcal{X}$.) Let $\underline{\mathcal{L}}$ be an $H$-equivariant line bundle on $\mathcal{B}_{e, 1}=Y$ as in 13.3. By [L5, 7.8(c)], for any $H$ equivariant line bundle $\mathcal{L}^{\prime}$ on $\mathcal{B}_{e, 1}$, the $R_{H}$-module $K_{H}\left(\mathcal{B}_{e, 1}\right)$ is generated by the elements $\theta_{x} \mathcal{L}^{\prime}$ with $x \in \mathcal{X}$. (It is here that the simply connectedness of $G$ is used.) In particular, this holds for $\mathcal{L}^{\prime}=\underline{\mathcal{L}}$. Hence from 14.9 we deduce the following result.

Theorem 14.11. The $\left(\mathcal{H}, R_{H}\right)$-module $K_{H}\left(\mathcal{B}_{e}\right)$ is generated by $\underline{\mathcal{L}}$.

Lemma 14.12. If $G=S L_{N}$, then condition 14.1(a) is automatically satisfied.

Let $G=S L(V)$, where $V$ is an $N$-dimensional vector space over C. Let $\epsilon$ : $V \rightarrow V$ be a nilpotent endomorphism. Define $a_{1}, a_{2}, a_{3}, \ldots$ by $a_{j}=\operatorname{dim} \operatorname{ker} \epsilon^{j}-$ $\operatorname{dim} \operatorname{ker} \epsilon^{j-1}$. Then $a_{1} \geq a_{2} \geq a_{3} \geq \ldots$ and $a_{1}+a_{2}+\cdots=N$. Consider a direct sum decomposition $V=V_{1} \oplus V_{2} \oplus \ldots$ with $\operatorname{dim} V_{j}=a_{j}$. For $j \leq 0$ we set $V_{j}=0$. Let $\mathfrak{q}^{\prime} \subset \mathfrak{s}_{\mathfrak{l}}(V)$ be the stabilizer of the filtration $V_{1} \subset V_{1} \oplus V_{2} \subset \ldots$ It is well known that $\epsilon$ belongs to the Richardson orbit in $\mathfrak{n}^{\prime}=\mathfrak{n}_{\mathfrak{q}^{\prime}}$. We grade $\mathfrak{g}$ by $\mathfrak{g}^{2 n}=$ $\left\{T \in \mathfrak{s l}(V) \mid T\left(V_{j+n}\right) \subset V_{j} \forall j\right\}$ and $\mathfrak{g}^{2 n+1}=0$. Then $\bigoplus_{n>0} \mathfrak{g}^{n}=\mathfrak{q}^{\prime}, \bigoplus_{n>0} \mathfrak{g}^{n}=\mathfrak{n}^{\prime}$. In $\mathfrak{g}^{2}$ we can find $T$ such that $T$ restricts to an injective map $V_{j+1} \rightarrow V_{j}$ for $j \in[1, n-1]$ and $T\left(V_{1}\right)=0$. We have $\operatorname{dim} \operatorname{ker} T=a_{1}, \operatorname{dim} \operatorname{ker} T^{2}=a_{1}+a_{2}$, etc. Thus $\operatorname{dim} \operatorname{ker} T^{j}=\operatorname{dim} \operatorname{ker} \epsilon^{j}$ for all $j$ hence $T \in \operatorname{Ad}(G) \epsilon . \operatorname{But} \operatorname{Ad}(G) \epsilon \cap \mathfrak{n}^{\prime}$ is the Richardson orbit in $\mathfrak{n}^{\prime}$. Thus, $T$ belongs to the Richardson orbit in $\mathfrak{n}^{\prime}$. By a known graded variant of the Morozov-Jacobson theorem, there exists $T^{\prime \prime} \in \mathfrak{g}^{-2}, T^{\prime} \in \mathfrak{g}^{0}$ such that $T, T^{\prime}, T^{\prime \prime}$ is an $s l_{2}$-triple. Note that $(e, h, f)$ is in the $G$-orbit of $T, T^{\prime}, T^{\prime \prime}$. Thus, condition 14.1(a) is verified. The lemma is proved. 


\section{RELAXING THE SIMPLY CONNECTEDNESS ASSUMPTION}

15.1. In this section we assume that $G$ is adjoint. Let $\pi: \tilde{G} \rightarrow G$ be a simply connected covering of $G$. We assume that $(e, h, f)$ satisfies conditions 13.1(b), 14.1(a), 13.7(a) relative to $\tilde{G}$. Let $\mathfrak{q}^{\prime}, \mathfrak{p}$ be as in $14.1,14.2$. What in 14.2 was denoted by $C$ will now be denoted by $\tilde{C}$, and we set $C=\pi(\tilde{C}), H=C \times \mathbf{C}^{*}, \tilde{H}=\tilde{C} \times \mathbf{C}^{*}$. The notation $\mathcal{X}, \mathcal{H}$ will refer to $G$; the analogous objects for $\tilde{G}$ will be denoted by $\tilde{\mathcal{X}}, \tilde{\mathcal{H}}$. Note that $\mathcal{X}$ may be naturally identified with a subgroup of $\tilde{\mathcal{X}}$ and $\mathcal{H}$ may be naturally identified with a subalgebra of $\tilde{\mathcal{H}}$.

The finite group $\underline{\mathcal{X}}=\tilde{\mathcal{X}} / \mathcal{X}$ acts on $W^{a}$ (see 9.1) by Coxeter group automorphisms $(\iota, w) \mapsto{ }^{\iota} w$ as in $[\mathrm{L} 5,1.3]$.

The results in $[\mathrm{L} 5, \S 1]$ give us an imbedding $\iota \mapsto \tilde{T}_{\iota}$ of $\underline{\mathcal{X}}$ into the group of invertible elements of $\tilde{\mathcal{H}}$ such that $\iota \otimes h \mapsto \tilde{T}_{\iota} h$ defines an isomorphism

$$
\mathcal{A}[\underline{\mathcal{X}}] \otimes_{\mathcal{A}} \mathcal{H} \stackrel{\sim}{\rightarrow} \tilde{\mathcal{H}}
$$

and $\tilde{T}_{\iota} \mathcal{H} \tilde{T}_{\iota}^{-1}=\mathcal{H}$ for all $\iota \in \underline{\mathcal{X}}$. More precisely, $\tilde{T}_{\iota} \tilde{T}_{s} \tilde{T}_{\iota}^{-1}=\tilde{T}_{\iota_{s}}$ for $s \in S$.

For any $x \in \tilde{\mathcal{X}}$ we denote by $\underline{x}$ the image of $x$ in $\underline{\mathcal{X}}$.

15.2. By 1.17(a), we have canonically

(a) $R_{\tilde{H}} \otimes_{R_{H}} K_{H}\left(\mathcal{B}_{e}\right)=K_{\tilde{H}}\left(\mathcal{B}_{e}\right)$.

In particular, $K_{H}\left(\mathcal{B}_{e}\right)$ may be naturally identified with a subgroup of $K_{\tilde{H}}\left(\mathcal{B}_{e}\right)$.

Let $\Gamma$ be the kernel of the canonical map $\tilde{H} \rightarrow H$ or equivalently the kernel of the canonical map $\tilde{C} \rightarrow C$. Let $\hat{\Gamma}=\operatorname{Hom}\left(\Gamma, \mathbf{C}^{*}\right)$. For any $\omega \in \hat{\Gamma}$ let $\hat{\tilde{C}}_{\omega}$ (resp. $\hat{\tilde{H}}_{\omega}$ ) be the set of characters of $\tilde{C}$ (resp. $\tilde{H}$ ) whose restriction to $\Gamma$ is $\omega$. We have a direct sum decomposition (as an $R_{H}$-module)

$$
R_{\tilde{H}}=\bigoplus_{\omega \in \hat{\Gamma}} R_{\tilde{H}, \omega}
$$

where $R_{\tilde{H}, \omega}$ is the subgroup of $R_{\tilde{H}}$ spanned by $\hat{\tilde{H}}_{\omega}$ (in particular, $R_{\tilde{H}, 1}=R_{H}$ ). Combining this with (a) we obtain a direct sum decomposition (as $\mathcal{H}$-modules)

(b) $K_{\tilde{H}}\left(\mathcal{B}_{e}\right)=\bigoplus_{\omega \in \hat{\Gamma}} K_{\tilde{H}}\left(\mathcal{B}_{e}\right)_{\omega}$ where $K_{\tilde{H}}\left(\mathcal{B}_{e}\right)_{\omega}=R_{\tilde{H}, \omega} K_{H}\left(\mathcal{B}_{e}\right)$ (in particular, $K_{\tilde{H}}\left(\mathcal{B}_{e}\right)_{1}=K_{H}\left(\mathcal{B}_{e}\right)$ ).

Let $x \mapsto[x]$ be the homomorphism $\tilde{\mathcal{X}} \rightarrow \hat{\tilde{C}}$ with kernel $\mathcal{X}_{K}$ defined as in 8.2 (for $\tilde{G}$ instead of $G$ ).

Let $x \mapsto[[x]]$ be the composition $\tilde{\mathcal{X}} \rightarrow \hat{\tilde{C}} \rightarrow \hat{\Gamma}$ where the first map is $x \mapsto[x]$ and the second one is restriction of characters.

Lemma 15.3. Let $x \in \tilde{\mathcal{X}}$. We have

(a) $[x] K_{\tilde{H}}\left(\mathcal{B}_{e}\right)_{\omega} \subset K_{\tilde{H}}\left(\mathcal{B}_{e}\right)_{\omega[[x]]}$;

(b) $\theta_{-x} K_{\tilde{H}}\left(\mathcal{B}_{e}\right)_{\omega} \subset K_{\tilde{H}}\left(\mathcal{B}_{e}\right)_{\omega[[-x]]}$;

(c) $\tilde{T}_{\underline{x}} K_{\tilde{H}}\left(\mathcal{B}_{e}\right)_{\omega} \subset K_{\tilde{H}}\left(\mathcal{B}_{e}\right)_{\omega[[-x]]}$.

(a),(b) follow from the definitions; (c) follows from (b) since, by [L5, 1.10], we have

(d) $\theta_{-x}=h_{x} \tilde{T}_{\underline{x}}$

where $h_{x} \in \mathcal{H}$ is invertible. 
15.4. Let $Y \subset \mathcal{B}_{e}$ be as in 13.3. We have $K_{\tilde{H}}(Y) \subset K_{\tilde{H}}\left(\mathcal{B}_{e}\right), K_{H}(Y) \subset K_{H}\left(\mathcal{B}_{e}\right)$ and the direct sum decomposition 15.2(b) induces a direct sum decomposition

(b) $K_{\tilde{H}}(Y)=\bigoplus_{\omega \in \hat{\Gamma}} K_{\tilde{H}}(Y)_{\omega}$ where $K_{\tilde{H}}(Y)_{\omega}=R_{\tilde{H}, \omega} K_{H}(Y)$ (in particular, $K_{\tilde{H}}(Y)_{1}=K_{H}(Y)$ ). The operators $[x], \theta_{x}: K_{\tilde{H}}\left(\mathcal{B}_{e}\right) \rightarrow K_{\tilde{H}}\left(\mathcal{B}_{e}\right)$ map $K_{\tilde{H}}(Y)$ into itself $(x \in \tilde{\mathcal{X}})$ and from 15.3 we deduce that

$$
\begin{aligned}
{[x] K_{\tilde{H}}(Y)_{\omega} } & \subset K_{\tilde{H}}(Y)_{\omega[[x]]}, \\
\theta_{x} K_{\tilde{H}}(Y)_{\omega} & \subset K_{\tilde{H}}(Y)_{\omega[[x]]} .
\end{aligned}
$$

for $x \in \tilde{\mathcal{X}}$. In particular, $[x] \theta_{-x}$ maps $K_{\tilde{H}}(Y)_{1}=K_{H}(Y)$ into itself for $x \in \tilde{\mathcal{X}}$.

Let $\mathcal{L}^{\prime}$ be any $H$-equivariant line bundle on $Y$. We shall need the following result.

Lemma 15.5. The $R_{H}$-module $K_{H}(Y)$ is generated by the elements $[x] \theta_{-x} \mathcal{L}^{\prime}$ for various $x \in \tilde{\mathcal{X}}$.

Let $\tilde{T}_{1}$ be a maximal torus of $\tilde{G}$ containing $\tilde{C}$ and contained in the parabolic subgroup of $\tilde{G}$ corresponding to $\mathfrak{q}^{\prime}$. Let $T_{1}=\pi\left(T_{1}\right)$. The $\tilde{H}$-action (resp. $H$-action) on $Y$ is the restriction of the natural action of $\tilde{T}_{1} \times \mathbf{C}^{*}$ (resp. $\left.T_{1} \times \mathbf{C}^{*}\right)$ on $Y$. It is then enough to prove a statement analogous to that of the lemma in which $H$ is replaced by $T_{1} \times \mathbf{C}^{*}$. That statement can be deduced from [L5, 7.8] (or rather, its obvious extension to the case of a reductive group with simply connected derived group).

Lemma 15.6. Let $\underline{\mathcal{L}} \in K_{H}\left(\mathcal{B}_{e}\right)$ be as in 13.3. The $\left(\mathcal{H}, R_{H}\right)$-module $K_{H}\left(\mathcal{B}_{e}\right)$ is generated by the elements $[x] \theta_{-x} \underline{\mathcal{L}}$ for various $x \in \tilde{\mathcal{X}}$.

This follows immediately from 14.9 and 15.5.

15.7. We define an action of $\tilde{\mathcal{X}}$ on $K_{\tilde{H}}\left(\mathcal{B}_{e}\right)$ by

(a) $x: \xi \mapsto x \circ \xi=[x]\left(\tilde{T}_{\underline{x}} \xi\right)$

where $\tilde{T}_{\underline{x}}$ acts by the $\tilde{\mathcal{H}}$-action and $[x]$ acts by the $R_{\tilde{H}}$-module structure. From 15.3 we see that this restricts to an action of $\tilde{\mathcal{X}}$ on $K_{H}\left(\mathcal{B}_{e}\right)$.

We can now state the following variant (for adjoint groups) of Theorem 14.11.

Proposition 15.8. Let $\underline{\mathcal{L}} \in K_{H}\left(\mathcal{B}_{e}\right)$ be as in 13.3. Then the elements $x \circ \underline{\mathcal{L}}$ (for various $x \in \tilde{\mathcal{X}}$ ) generate the $\mathcal{H}$-module $K_{H}\left(\mathcal{B}_{e}\right)$.

Let $\xi \in K_{H}\left(\mathcal{B}_{e}\right)$. By 15.6 we have

$$
\xi=\sum_{x \in \tilde{\mathcal{X}}, x^{\prime} \in \mathcal{X}} h_{x, x^{\prime}}\left[x^{\prime}\right][x] \theta_{-x} \underline{\mathcal{L}}
$$

where $h_{x, x^{\prime}} \in \mathcal{H}$ are 0 for all but finitely many $x, x^{\prime}$. Using now $15.3(\mathrm{~d})$, we obtain

$$
\begin{aligned}
\xi & =\sum_{x \in \tilde{\mathcal{X}}, x^{\prime} \in \mathcal{X}} h_{x, x^{\prime}}\left[x^{\prime}\right][x] h_{x} \tilde{T}_{\underline{x}} \underline{\mathcal{L}}=\sum_{x \in \tilde{\mathcal{X}}, x^{\prime} \in \mathcal{X}} h_{x, x^{\prime}} h_{x}\left[x^{\prime}\right][x] \tilde{T}_{\underline{x}} \underline{\mathcal{L}} \\
& =\sum_{x \in \tilde{\mathcal{X}}, x^{\prime} \in \mathcal{X}} h_{x, x^{\prime}} h_{x}\left[x^{\prime}+x\right] \tilde{T}_{x+x^{\prime}} \underline{\mathcal{L}},
\end{aligned}
$$

since $\underline{x}^{\prime}=0$. The proposition is proved. 
15.9. For $x \in \tilde{\mathcal{X}}$, the map $\tilde{t}_{x}: \xi \mapsto x \circ \xi$ of $K_{H}\left(\mathcal{B}_{e}\right)$ into itself is $R_{H}$-linear and $\mathcal{H}$ semilinear with respect to the automorphism of $\mathcal{H}$ given by conjugation by $\tilde{T}_{\underline{x}}$ in $\tilde{\mathcal{H}}_{\text {. }}$ Hence it extends to an $\mathfrak{R}$-linear map $\tilde{t}_{x}$ of $\mathfrak{E}_{e}$ into itself which is again $\mathcal{H}$-semilinear as above.

15.10. In the setup of 9.1 , we may identify $\tilde{\mathcal{X}}$ with the set of vectors $x \in \mathcal{V}$ such that $\check{\alpha}_{i}(x) \in \mathbf{Z}$ for any $i \in I$. The definition of the action $x \mapsto \gamma_{x}$ of $\mathcal{X}$ on $\Xi$ and $X_{\Xi}$ (see 9.2) extends without change to a definition of an action $x \mapsto \gamma_{x}$ of $\tilde{\mathcal{X}}$ on $\Xi$ and $X_{\Xi}$. For $x \in \tilde{\mathcal{X}}$ we define an $\mathcal{A}$-linear map $t_{x}: \mathbf{M}_{c} \rightarrow \mathbf{M}_{c}$ by $t_{x}(A)=(-1)^{\mu_{K}^{\prime}(x)} \gamma_{x}(A)$. This is well defined since $\mu_{K}^{\prime}(x) \in \mathbf{Z}$, by $8.3(\mathrm{~b})$. Note that $x \mapsto t_{x}$ is an action of $\tilde{\mathcal{X}}$. The map $t_{x}: \mathbf{M}_{c} \rightarrow \mathbf{M}_{c}$ is $R_{H}$-linear and $\mathcal{H}$ semilinear with respect to the automorphism of $\mathcal{H}$ given by conjugation by $\tilde{T}_{\underline{x}}$ in $\tilde{\mathcal{H}}$. Hence it extends to an $\mathfrak{R}$-linear map $t_{x}$ of $\left(\mathbf{M}_{c}\right)_{\mathfrak{R}}$ into itself which is again $\mathcal{H}$-semilinear as above. Using the (absolute) irreducibility of the $\mathcal{H}$-module $\left(\mathbf{M}_{c}\right)_{\mathfrak{R}}$, it then follows that the map $\Phi \tilde{t}_{x} \Phi^{-1}:\left(\mathbf{M}_{c}\right)_{\mathfrak{R}} \rightarrow\left(\mathbf{M}_{c}\right)_{\mathfrak{R}}$ must be a multiple of the map $\gamma_{x}:\left(\mathbf{M}_{c}\right)_{\mathfrak{R}} \rightarrow\left(\mathbf{M}_{c}\right)_{\mathfrak{R}}$. Thus, $\Phi t_{x} \Phi^{-1}=f_{x} t_{x}$ for some $f_{x} \in \mathfrak{R}-\{0\}$. Clearly, $x \mapsto f_{x}$ is a homomorphism $\tilde{\mathcal{X}} \rightarrow \mathfrak{R}-\{0\}$. For $x \in \mathcal{X}$ both the action $t_{x}$ and $\tilde{t}_{x}$ are given by the $R_{H}$-module structures. Hence $f_{x}=1$ for $x \in \mathcal{X}$. Thus, $x \mapsto f_{x}$ factors through a homomorphism $\tilde{\mathcal{X}} / \mathcal{X} \rightarrow \mathfrak{R}-\{0\}$. Since $\tilde{\mathcal{X}} / \mathcal{X}$ is finite and $\mathfrak{R}-\{0\}$ contains no roots of 1 other than \pm 1 , it follows that $f_{x}= \pm 1$ for any $x \in \tilde{\mathcal{X}}$.

15.11. Let $\mathbf{M}_{c}^{\prime}$ be the $\mathcal{H}$-submodule of $\mathbf{M}_{c}$ generated by the elements

$$
t_{x}\left(\sum_{t \in W^{!}} v^{l(t)}\left({ }^{(t w)^{-1}} A_{0}^{+}\right)\right)
$$

where $x$ runs over $\tilde{X}$ and $w$ is as in $13.11(\mathrm{a})$. Let $\mathbf{M}_{c}^{\prime \prime}$ be the set of all $m^{\prime} \in\left(\mathbf{M}_{c}\right)_{\mathfrak{R}}$ such that $\left(m_{1} \| m^{\prime}\right) \in R_{H}$ for all $m_{1} \in \mathbf{M}_{c}^{\prime}$.

The following result describes $K_{H}\left(\mathcal{B}_{e}\right), K_{H}\left(\Lambda_{e}\right)$ in an almost combinatorial way.

Theorem 15.12. (a) $\Phi$ maps $K_{H}\left(\mathcal{B}_{e}\right)$ isomorphically onto $\mathbf{M}_{c}^{\prime}$.

(b) $\Phi$ maps $K_{H}\left(\Lambda_{e}\right)$ isomorphically onto $\mathbf{M}_{c}^{\prime \prime}$.

(a) follows from $15.8,15.10$, and the results in $\S 13$. To prove (b), it is enough to show that the pairing $(\|): K_{H}\left(\mathcal{B}_{e}\right) \times K_{H}\left(\Lambda_{e}\right) \rightarrow R_{H}$ is perfect. This follows from $8.1(\mathrm{a})$, since $(\|)$ is obtained by twisting $(:)$ using some transformations which are invertible over $R_{H}$. The theorem is proved.

15.13. In addition to the assumptions in 15.11 we make the following assumption:

(a) For any $B \in X_{\Xi}$ we have $B_{\leq} \in \mathbf{M}_{c}^{\prime}$; moreover, $\left\{B_{\leq} \mid B \in X_{\Xi}\right\}$ is an $\mathcal{A}$-basis of $\mathbf{M}_{c}^{\prime}$.

(It is likely that (a) follows automatically from the assumptions in 15.11.) We show that, in this case, Conjecture 5.12 holds for $(e, h, f)$ except possibly for 5.12 $(g)$. Using 15.12(a), 10.7(a),(c), we see that $\mathbf{B}_{\mathcal{B}_{e}}^{ \pm}=\Phi^{-1}\{ \pm B \leq \mid B \in X\}$ and that this is a signed basis of the $\mathcal{A}$-module $K_{H}\left(\mathcal{B}_{e}\right)$. Thus, 5.12(a) holds in our case. By 12.9, we have $B_{\geq} \in \mathbf{M}_{c}$ hence $\hat{B_{\geq}} \in\left(z^{\dagger}\right)^{-1} \mathbf{M}_{c}$ for all $B$. By (a) and 11.7(a), $\left\{\hat{B_{\geq}} \mid B \in X_{\Xi}\right\}$ is an $\mathcal{A}$-basis of $\mathbf{M}_{c}^{\prime \prime}$. Using $15.12(\mathrm{~b}), 10.7(\mathrm{~b}),(\mathrm{d})$, we see that $\mathbf{B}_{\Lambda_{e}}^{ \pm}=\Phi^{-1}\left\{ \pm B_{\geq} \mid B \in X\right\}$ and that this is a signed basis of the $\mathcal{A}$-module $K_{H}\left(\Lambda_{e}\right)$. Thus, 5.12(b) holds in our case. The argument above also yields $5.12(\mathrm{e})$,(f). Now $5.12(\mathrm{c}),(\mathrm{d})$ follow from $10.10 ; 5.12(\mathrm{~h})$ holds by 8.20 . 


\section{EXAMPLES}

16.1. By [L6], Conjecture 5.12 holds for $e$ subregular nilpotent in $G$ of type $D_{n}$ or $E_{n}$. In this section we give a number of examples where the arguments in 15.13 can be applied, yielding most of the statements of 5.12. In these examples $G$ is adjoint and $(e, h, f)$ is as in 8.1.

16.2. In this subsection we assume that $e=h=f=0$. Conditions 13.1(b), 14.1(a) are trivially satisfied; we may take $K=\emptyset, K^{!}=I$. Condition 13.7(a) is also satisfied. Condition 15.13(a) holds by results in [L1]. Hence by 15.13 , conjecture 5.12 holds in this case except possibly for $5.12(\mathrm{~g})$.

16.3. Assume now that $k$ is an integer $\geq 1$ and $V$ is a $\mathbf{C}$-vector space of dimension $N \geq 2 k$. Let $G=P G L(V)$ so that $\mathfrak{g}=\mathfrak{s l}(V)$. Assume that $(e, h, f)$ is as in 8.1 such that $e$ has exactly two Jordan blocks, of sizes $k, N-k$. Note that the assumptions of 15.1 are satisfied in this case. (See 14.12.)

In this case, the alcoves in $X_{\Xi}$ can be indexed by sequences $\left(a_{i}\right)_{i \in \mathbf{Z}}$ with $a_{i} \in \mathbf{Z}$ such that

(a) $a_{i}<a_{i+1}$ and $a_{i+k}=a_{i}+N$ for all $i$.

Let $A_{\left(a_{i}\right)}$ be the alcove corresponding to $\left(a_{i}\right)$. Moreover, we may identify $S=\mathbf{Z} / N \mathbf{Z}$ so that the $\mathcal{H}$-module structure on $\mathbf{M}_{c}$ is described as follows.

Assume that $\left(a_{i}\right),\left(a_{i}^{\prime}\right), n$ are such that for some residue class $\gamma$ (modulo $k$ ) in $\mathbf{Z}$ we have

(b) $a_{i}^{\prime}=a_{i}+1, a_{i}=n \bmod N$ if $i \in \gamma$ and $a_{i}^{\prime}=a_{i}$ if $i \notin \gamma$.

Then $\tilde{T}_{n} A_{\left(a_{i}\right)}=A_{\left(a_{i}^{\prime}\right)}$ and $\tilde{T}_{n} A_{\left(a_{i}^{\prime}\right)}=A_{\left(a_{i}\right)}+\left(v-v^{-1}\right) A_{\left(a_{i}^{\prime}\right)}$.

Assume that $\left(a_{i}\right), n$ are such that there is no $\left(a_{i}^{\prime}\right)$ so that (b) holds. Then $\tilde{T}_{n} A_{\left(a_{i}\right)}=-v^{-1} A_{\left(a_{i}\right)}$

Assume that $\left(a_{i}^{\prime}\right), n$ are such that there is no $\left(a_{i}\right)$ so that (b) holds. Then $\tilde{T}_{n} A_{\left(a_{i}^{\prime}\right)}=-v^{-1} A_{\left(a_{i}^{\prime}\right)}$

For any $A \in X_{\Xi}$, the elements $A_{\leq}$can be computed explicitly, at least if $k \in$ $\{1,2,3\}$. The result is as follows.

Case $k=1$. Let $A=A_{\left(a_{i}\right)}$. Then

$$
A_{\leq}=A_{\left(\ldots a_{0} \ldots\right)}+v^{-1} A_{\left(\ldots a_{0}-1 \ldots\right)} .
$$

Case $k=2$. Let $A=A_{\left(a_{i}\right)}$ be such that $a_{i+1}-a_{i} \geq 2$ for all $i$. Then

$$
A_{\leq}=A_{\left(\ldots a_{0}, a_{1} \ldots\right)}+v^{-1} A_{\left(\ldots a_{0}-1, a_{1} \ldots\right)}+v^{-1} A_{\left(\ldots a_{0}, a_{1}-1 \ldots\right)}+v^{-2} A_{\left(\ldots a_{0}-1, a_{1}-1 \ldots\right)} .
$$

Let $A=A_{\left(a_{i}\right)}$ be such that $a_{i}=a, a_{i+1}=a+1$ for some $i$. Then

$$
A_{\leq}=A_{(\ldots a, a+1 \ldots)}+v^{-1} A_{(\ldots a-1, a+1 \ldots)}+v^{-1} A_{(\ldots a-2, a \ldots)}+v^{-2} A_{(\ldots a-2, a-1 \ldots)} .
$$

Case $k=3$. Let $A=A_{\left(a_{i}\right)}$ be such that $a_{i+1}-a_{i} \geq 2$ for all $i$. Then

$$
\begin{aligned}
& A_{\leq}=A_{\left(\ldots a_{0}, a_{1}, a_{2} \ldots\right)}+v^{-1} A_{\left(\ldots a_{0}-1, a_{1}, a_{2} \ldots\right)}+v^{-1} A_{\left(\ldots a_{0}, a_{1}-1, a_{2} \ldots\right)} \\
& +v^{-1} A_{\left(\ldots a_{0}, a_{1}, a_{2}-1 \ldots\right)}+v^{-2} A_{\left(\ldots a_{0}-1, a_{1}-1, a_{2} \ldots\right)}+v^{-2} A_{\left(\ldots a_{0}, a_{1}-1, a_{2}-1 \ldots\right)} \\
& +v^{-2} A_{\left(\ldots a_{0}-1, a_{1}, a_{2}-1 \ldots\right)}+v^{-3} A_{\left(\ldots a_{0}-1, a_{1}-1, a_{2}-1 \ldots\right) .}
\end{aligned}
$$


Let $A=A_{\left(a_{i}\right)}$ be such that for some $i, a_{i}=a, a_{i+1}=a+1, a_{i-1}=b \leq a-3, a_{i+2} \geq$ $a+3$. Then

$$
\begin{aligned}
& A_{\leq}=A_{(\ldots b, a, a+1 \ldots)}+v^{-1} A_{(\ldots b-1, a, a+1 \ldots)}+v^{-1} A_{(\ldots b, a-1, a+1 \ldots)} \\
& +v^{-1} A_{(\ldots b, a-2, a \ldots)}+v^{-2} A_{(\ldots b-1, a-1, a \ldots)}+v^{-2} A_{(\ldots b, a-2, a-1 \ldots)} \\
& +v^{-2} A_{(\ldots b-1, a-2, a \ldots)}+v^{-3} A_{(\ldots b-1, a-2, a-1 \ldots)} .
\end{aligned}
$$

Let $A=A_{\left(a_{i}\right)}$ be such that for some $i, a_{i}=a, a_{i+1}=a+1, a_{i-1}=a-2, a_{i+2} \geq a+3$. Then

$$
\begin{aligned}
& A_{\leq}=A_{(\ldots a-2, a, a+1 \ldots)}+v^{-1} A_{(\ldots a-3, a, a+1 \ldots)}+v^{-1} A_{(\ldots a-2, a-1, a+1 \ldots)} \\
& +v^{-1} A_{(\ldots a-4, a-2, a \ldots)}+v^{-2} A_{(\ldots a-3, a-1, a+1 \ldots)}+v^{-2} A_{(\ldots a-4, a-2, a-1 \ldots)} \\
& +v^{-2} A_{(\ldots a-4, a-3, a \ldots)}+v^{-3} A_{(\ldots a-4, a-3, a-1 \ldots)} .
\end{aligned}
$$

Let $A=A_{\left(a_{i}\right)}$ be such that for some $i, a_{i-1}=a-1, a_{i}=a, a_{i+1}=a+1$. Then

$$
\begin{aligned}
& A_{\leq}=A_{(\ldots a-1, a, a+1 \ldots)}+v^{-1} A_{(\ldots a-2, a, a+1 \ldots)}+v^{-1} A_{(\ldots a-3, a-1, a+1 \ldots)} \\
& +v^{-1} A_{(\ldots a-4, a-1, a \ldots)}+v^{-2} A_{(\ldots a-3, a-2, a+1 \ldots)}+v^{-2} A_{(\ldots a-4, a-2, a \ldots)} \\
& +v^{-2} A_{(\ldots a-4, a-3, a-1 \ldots)}+v^{-3} A_{(\ldots a-4, a-3, a-2 \ldots)} .
\end{aligned}
$$

In particular, $A_{\leq} \in \mathbf{M}_{c}$ for all $A$. Using the formulas above one can check that we also have $A_{\leq} \in \mathbf{M}_{c}^{\prime}$ (notation of 15.11). Hence the arguments in 15.13 are applicable, so that conjecture 5.12 holds in this case except possibly for $5.12(\mathrm{~g})$.

16.4. Let $V$ be a $\mathbf{C}$-vector space of dimension $2 N \geq 6$ with a given symmetric perfect bilinear pairing $():, V \times V \rightarrow \mathbf{C}$. Assume that $G=S O(V) / \pm 1$, so that $\mathfrak{g}=\mathfrak{s} \mathfrak{o}(V)$. We list the elements of $I$ as

$$
\begin{array}{lrrrr}
1 & 2 & 3 & \ldots & N-1 \\
1^{\prime} & & &
\end{array}
$$

where $\sigma_{i}, \sigma_{j}$ do not commute if $i, j$ are adjacent elements in the same row or column and $\sigma_{i}, \sigma_{j}$ commute, otherwise. Let $(e, h, f)$ be as in 8.1, such that with the notation of 8.1 , we have $K=I-\{N-1\}$. Then $e: V \rightarrow V$ has one Jordan block of size $2 N-3$ and 3 Jordan blocks of size 1. Thus $e$ satisfies condition 13.1(b).

We show that $(e, f, h)$ satisfies condition 14.1(a). Consider a direct sum decomposition

$$
V=V_{-N+2} \oplus V_{-N+3} \oplus \ldots \oplus V_{-1} \oplus V_{0} \oplus V_{1} \oplus \ldots \oplus V_{N-2},
$$

where $\operatorname{dim} V_{0}=4, \operatorname{dim} V_{k}=1$ for $\pm k \in[1, N-2]$, such that $\left(V_{k}, V_{k^{\prime}}\right)=0$ for $k+k^{\prime} \neq 0$. We set $V_{k}=0$ for $|k|>N-2$. We define

$$
\begin{gathered}
\mathfrak{g}^{2 n}=\left\{T \in \mathfrak{s o}(V) \mid T\left(V_{j+n}\right) \subset V_{j} \quad \forall j\right\}, \\
\mathfrak{g}^{2 n+1}=0 .
\end{gathered}
$$

Then $\mathfrak{g}=\bigoplus_{n} \mathfrak{g}^{n}$ is a grading of the Lie algebra $\mathfrak{g}$ and $\mathfrak{q}^{\prime}=\bigoplus_{n \geq 0} \mathfrak{g}^{n}$ is a parabolic subalgebra. It consists of all $T \in \mathfrak{g}$ such that, for any $k, T$ maps $V_{1} \oplus V_{2} \oplus \ldots \oplus V_{k}$ into itself. The nil-radical of $\mathfrak{q}^{\prime}$ is $\mathfrak{n}^{\prime}=\bigoplus_{n \geq 0} \mathfrak{g}^{n}$. It consists of all $T \in \mathfrak{g}$ such that, for any $k, T$ maps $V_{1} \oplus V_{2} \oplus \ldots \oplus V_{k}$ into $V_{1} \oplus V_{2} \oplus \ldots \oplus V_{k-1}$. It is easy to see that $e$ belongs to the Richardson orbit in $\mathfrak{n}^{\prime}$.

In $\mathfrak{g}^{2}$ we can find $T \in \mathfrak{s o}(V)$ such that the following holds: there exists an orthogonal direct sum decomposition $V_{0}=V_{0}^{\prime} \oplus V_{0}^{\prime \prime}$ with $\operatorname{dim} V_{0}^{\prime}=1, \operatorname{dim} V_{0}^{\prime \prime}=3$ such that $T\left(V_{0}^{\prime \prime}\right)=0$ and $T$ restricts to isomorphisms $V_{j+1} \stackrel{\sim}{\rightarrow} V_{j}$ for $j \in[-N+$ $2,-2] \cup[1, N-3]$ and $V_{1} \stackrel{\sim}{\rightarrow} V_{0}^{\prime} \stackrel{\sim}{\rightarrow} V_{-1}$. 
Clearly, $T \in \operatorname{Ad}(G) e$. But $\operatorname{Ad}(G) e \cap \mathfrak{n}^{\prime}$ is the Richardson orbit in $\mathfrak{n}^{\prime}$. Thus, $T$ belongs to the Richardson orbit in $\mathfrak{n}^{\prime}$. By a known graded variant of the MorozovJacobson theorem, there exists $T^{\prime \prime} \in \mathfrak{g}^{-2}, T^{\prime} \in \mathfrak{g}^{0}$ such that $T, T^{\prime}, T^{\prime \prime}$ is an $\mathfrak{s l}_{2}$-triple. Note that $(e, h, f)$ is in the $G$-orbit of $T, T^{\prime}, T^{\prime \prime}$. Thus, condition 14.1(a) is verified.

With the notation of $\S 13$, we have $K^{!}=\left\{1,1^{\prime}\right\}$. It is easy to see that $K, K^{!}$ satisfy the condition 13.7 (a). Thus the assumptions of 15.1 are verified in our case.

The alcoves in $X_{\Xi}$ can be labelled as

$$
A_{n},(n \in \mathbf{Z}) \text {, and } A_{n}^{\prime},(n \in \mathbf{Z}, n=1 \bmod N-1),
$$

in such a way that $A_{n} \leq A_{n+1}$ for any $n$ and $A_{n-1} \leq A_{n}^{\prime} \leq A_{n+1}$ for any $n=1$ $\bmod N-1$.

The elements $B_{\leq}$can be computed for any alcove $B$. They are given

$$
\begin{aligned}
& \left(A_{n}\right)_{\leq}=A_{n}+v^{-1} A_{n-1}+v^{-1} A_{n-2 N+3}+v^{-2} A_{n-2 N+2}^{\prime}, \\
& \left(A_{n}^{\prime}\right)_{\leq}=A_{n}^{\prime}+v^{-1} A_{n-1}+v^{-1} A_{n-2 N+3}+v^{-2} A_{n-2 N+2},
\end{aligned}
$$

if $n=1 \bmod N-1$,

$$
\left(A_{n}\right)_{\leq}=A_{n}+v^{-1} A_{n-1}+v^{-1} A_{n-1}^{\prime}+v^{-2} A_{n-2},
$$

if $n=2 \bmod N-1$,

$$
\left(A_{n}\right)_{\leq}=A_{n}+v^{-1} A_{n-1}+v^{-1} A_{n^{\prime \prime}+1}+v^{-2} A_{n^{\prime \prime}},
$$

if $2+(p-1)(N-1)<n<1+p(N-1)$, and $n^{\prime \prime}$ is given by $\left(n^{\prime \prime}+n\right) / 2=$ $1+(p-1)(N-1)$. In particular, $B_{\leq} \in \mathbf{M}_{c}$. Using the formulas above one can check that we also have $B_{\leq} \in \mathbf{M}_{c}^{\prime}$ (notation of 15.11). Hence the arguments in 15.13 are applicable, so that Conjecture 5.12 holds in this case except possibly for $5.12(\mathrm{~g})$.

16.5. The elements $B_{\geq}$can be computed for any alcove $B$. They are given by:

$$
\begin{aligned}
& \left(A_{n}\right)_{\geq}=A_{n}+\sum_{t \in[1, N-2]}(-v)^{-t} A_{n+t}+(-v)^{-N+1}\left(A_{n+N-1}+A_{n+N-1}^{\prime}\right) \\
& +\sum_{t \in[1, N-2]}(-v)^{-t-N+1} A_{n+N-1+t}+(-v)^{-2 N+2} A_{n+2 N-2}^{\prime}, \\
& \left(A_{n}^{\prime}\right)_{\geq}=A_{n}^{\prime}+\sum_{t \in[1, N-2]}(-v)^{-t} A_{n+t}+(-v)^{-N+1}\left(A_{n+N-1}+A_{n+N-1}^{\prime}\right) \\
& +\sum_{t \in[1, N-2]}(-v)^{-t-N+1} A_{n+N-1+t}+(-v)^{-2 N+2} A_{n+2 N-2},
\end{aligned}
$$

if $n=1 \bmod N-1$,

$$
\begin{aligned}
& \left(A_{n}\right)_{\geq}=\sum_{\substack{t \in[0,2 N-3] \\
+}}(-v)^{-t} A_{n+t}+\sum_{\substack{t \in[0,2 N-3] \\
n+t=1, \bmod N-1}}(-v)^{-t} A_{n+t}^{\prime}+\sum_{\substack{t \in[1,2 N-2] \\
n^{\prime}+t=1 \bmod N-1}}(-v)^{-t} A_{n^{\prime}+t} \\
& +\sum^{-t} A_{n^{\prime}+t}^{\prime},
\end{aligned}
$$

if $1+(p-1)(N-1)<n<1+p(N-1)$ and $n^{\prime}$ is given by $\left(n^{\prime}+n\right) / 2=1+p(N-1)$.

Using these formulas and 10.9 we see that Conjecture $5.12(\mathrm{~g})$ holds in our case. 


\section{Conjectures}

17.1. We return to the setting in 1.8. We assume that $G$ is of adjoint type. Let $\left(W^{a}, S\right), \tilde{T}_{w} \in \mathcal{H}^{-}: \mathcal{H} \rightarrow \mathcal{H}$ be as in 9.1. For any $w \in W^{a}$, let $c_{w}^{\prime}$ be the unique element of $\mathcal{H}$ such that $\bar{c}_{w}^{\prime}=c_{w}^{\prime}$ and $c_{w}^{\prime}=\tilde{T}_{w} \bmod v^{-1} \sum_{y \in W^{a}} \mathbf{Z}\left[v^{-1}\right] \tilde{T}_{y}$. Let $a: W^{a} \rightarrow \mathbf{N}$ be the function defined in [L3, I]. Consider the largest number $a_{0}$ such that $c_{w}^{\prime}: K_{H}\left(\mathcal{B}_{e}\right) \rightarrow K_{H}\left(\mathcal{B}_{e}\right)$ is non-zero for some $w \in W^{a}$ with $a(w)=a_{0}$. It is known [L3, IV] that the two sided cell $\underline{c}$ containing such a $w$ is well defined (it depends only on $e$ ) and that $a_{0}=b(e)$. For any right cell $R$ contained in $\underline{c}$, we set $K_{H}\left(\mathcal{B}_{e}\right)_{\mathfrak{R}}^{R}=\sum_{w \in R} c_{w}^{\prime}\left(K_{H}\left(\mathcal{B}_{e}\right)_{\mathfrak{R}}\right)$. According to [L3, III, 3.7], we have a direct sum decomposition

(a) $K_{H}\left(\mathcal{B}_{e}\right)_{\mathfrak{R}}=\bigoplus_{R}\left(K_{H}\left(\mathcal{B}_{e}\right)_{\mathfrak{R}}^{R}\right)$

where $R$ runs over the right cells in $\underline{c}$. We conjecture that

(b) any element of $\mathbf{B}_{\mathcal{B}_{e}}^{ \pm}$is contained in one of the summands in (a).

This would give us a map

(c) $\mathbf{B}_{\mathcal{B}_{e}}^{ \pm} \rightarrow$ set of right cells in $\underline{c}$

If we assume 5.12(a) and 5.16(a) we see (by 5.17) that (c) factors through a surjective map

(d) $\underline{\mathbf{B}}_{\mathcal{B}_{e}}^{ \pm} \rightarrow$ set of right cells in $\underline{c}$.

We conjecture that the finite set $\underline{\mathbf{B}}_{\mathcal{B}_{e}}^{ \pm}$(which carries a natural action of the (finite) group of components of the centralizer of $e$ ) is the set $Y$ which appears in [L3, IV, 10.5]. In particular, we expect that the orbits of the finite group above are precisely the fibres of the map (d).

17.2. Assume that $G$ is adjoint. Let $\mathfrak{g}^{\prime}$ be the Lie algebra of an adjoint algebraic group of the same type as $G$, but over an algebraic closure $\mathbf{k}$ of the field with $p$ elements ( $p$ sufficiently large). Let $\chi$ be a nilpotent element in $\mathfrak{g}^{\prime}$ of the same type as $e \in \mathfrak{g}$. Let $\mathcal{C}_{\chi}$ be the abelian category considered in [L5, 14.1]. Its objects are modules over a k-algebra $U_{\chi}$ of dimension of $p^{\text {dimg }} \mathfrak{g}$ wich are at the same time modules over a torus with the same character group as $C$. We fix a generic block of this category. Let $\mathbf{I}$ be an indexing set for the simple objects in this block. Note that $\hat{C}$ acts naturally and freely on $\mathbf{I}$. For $\mathbf{i} \in \mathbf{I}$, let $L_{\mathbf{i}}$ be the corresponding simple object of $\mathcal{C}_{\chi}$ and let $Q_{\mathbf{i}}$ be the indecomposable projective cover of $L_{\mathbf{i}}$. For $\mathbf{i} \in \mathbf{I}$ we have $Q_{\mathbf{i}}=\sum_{\mathbf{i}^{\prime} \in \mathbf{I}} n_{\mathbf{i}_{\mathbf{i}} \mathbf{i}^{\prime}} L_{\mathbf{i}^{\prime}}$ in the appropriate Grothendieck group. Here $n_{\mathbf{i}, \mathbf{i}^{\prime}} \in \mathbf{N}$ are 0 for all but finitely many $\mathbf{i}^{\prime}$.

We conjecture (cf. $[\mathrm{L} 7,2.4]$ ) that there exists a bijection $\zeta: \mathbf{I} \stackrel{\sim}{\rightarrow} \mathbf{B}_{\Lambda_{e}}^{ \pm} / \pm 1$ which is compatible with the natural free $\hat{C}$-actions on the two sets, such that

$$
n_{\mathbf{i}, \mathbf{i}^{\prime}}=\left|\partial\left(\nabla_{e} \zeta(\mathbf{i})|| \zeta\left(\mathbf{i}^{\prime}\right)\right)_{v=-1}\right|
$$

for all $\mathbf{i}, \mathbf{i}^{\prime} \in \mathbf{I}$. (This conjecture is a revised form of $[\mathrm{L} 5,14.5]$.)

The category $\mathcal{C}_{\chi}$ admits a quantum analogue (in characteristic 0 ) in which instead of $\mathfrak{g}^{\prime}$ one uses a quantum group (as in [DK]) at a root of 1 . I expect that exactly the same formalism as in the conjecture above applies in this quantum case.

17.3. Assume now that $(e, h, f), \mathfrak{l}, W^{*}$ are as in 8.1. In this case, we can assume that $\mathbf{I}=X_{\Xi}\left(\right.$ as in 9.2, 9.4). Hence $L_{A}, Q_{A}$ are defined for $A \in X_{\Xi}$ and $n_{A, A^{\prime}} \in \mathbf{N}$ are defined for $A, A^{\prime} \in X_{\Xi}$. Moreover, we can consider for each $A \in X_{\Xi}$, the corresponding "baby Verma module" $Z_{A}$ with simple quotient $L_{A}$. We conjecture that 
(a) $\zeta: X_{\Xi} \stackrel{\sim}{\rightarrow} \mathbf{B}_{\Lambda_{e}}^{ \pm} / \pm$is given by $\Phi(\zeta(B))= \pm \hat{B_{\geq}}$(see 10.7(b)),

(b) $Z_{A}=\sum_{B^{\prime} ; A>B^{\prime}} \pi_{B^{\prime}, A}(-1) L_{B^{\prime}}$ and

(c) $Q_{B}=\sum_{A ; A \geq B}\left|W^{*}\right| \pi_{B, A}(-1) Z_{A}$,

for any $A, B \in X_{\Xi}$ in the Grothendieck group of our block. Here $\pi_{B, A}$ is as in $9.17(\mathrm{~d})$. (In particular, the sums (b),(c) should have only finitely many non-zero terms.)

We show that this conjecture implies the conjecture 17.2(a) (in the present case). Indeed, assuming that (a),(b),(c) hold, we have

$$
Q_{B}=\sum_{B^{\prime}}\left|W^{*}\right| \sum_{A ; A \geq B ; A \geq B^{\prime}} \pi_{B, A}(-1) \pi_{B^{\prime}, A}(-1) L_{B^{\prime}} .
$$

We note that $\left|W^{*}\right|=\nabla_{e, \mathrm{r}}(-1)$ and we use 10.9 ; we obtain

$$
Q_{B}=\sum_{B^{\prime}}\left|\partial\left(\nabla_{e} \zeta(B)|| \zeta\left(B^{\prime}\right)\right)_{v=-1}\right| L_{B^{\prime}}
$$

so that 17.2 (a) holds.

\section{INDEX OF NOTATION}

0.2. $G, \mathfrak{g}, \mathfrak{g}_{n i l}, \mathcal{B}, \nu, \Lambda^{\prime}, \Lambda, \mathfrak{n}_{\mathfrak{p}}, I, \mathbf{X}, \mathcal{X}, L_{x}, \alpha_{i}, \check{\alpha}_{i}, \mathcal{X}_{\geq}, \mathcal{X}_{\leq}, \sigma_{i}, W, l: W \rightarrow \mathbf{N}, \mathcal{A}, \mathcal{H}$

1.1. $\chi_{X}, A_{*}(X), \operatorname{Coh}_{H}(X), \operatorname{Vec}_{H}(X), K_{H}(X), K_{H}^{i}(X), R_{H}$

1.8. $e, h, f, \phi, \mathfrak{z}_{\mathfrak{m}}(f), \mathcal{B}_{e}, \Sigma_{e}^{\prime}, \Sigma_{e}, \Lambda_{e}^{\prime}, \Lambda_{e}, C, H, \mathfrak{R}$

1.15. $\mathfrak{E}_{e}$

2.4. (:)

3.1. $V, \Omega_{V},^{\dagger}: R_{H} \rightarrow R_{H}{ }^{-}: R_{H} \rightarrow R_{H}, \mathfrak{t}, \nabla_{e}, \nabla_{e}^{\prime}$

4.2. $\mathcal{O}_{\sigma_{i}}, \overline{\mathcal{O}}_{i}, \bar{Z}_{i}$

5.1. $I_{e}, b(e)$

5.7. $\Upsilon$

5.8. $(\|)$

5.9. $\partial$

5.11. $\tilde{\beta}, \beta, D_{\mathcal{B}_{e}}, D_{\Lambda_{e}}, \mathbf{B}_{\mathcal{B}_{e}}^{ \pm}, \mathbf{B}_{\Lambda_{e}}^{ \pm}$

6.1. p , n, $P, L, U, \Pi^{\prime}, \Pi, \tilde{\Pi}, \mathfrak{Z}, \tilde{\mathfrak{Z}}, K, W^{*}, W_{*}, \underline{\mathcal{B}}_{e}, \underline{\Lambda}_{e}, \mathfrak{b}^{!}$

7.9. $\underline{\mathcal{H}}$

8.1. $\varpi$

8.2. $\mathcal{X}_{K}$

8.3. $\mu_{K}^{\prime}$

8.15. $z$

9.1. $\mathcal{V}, \mathcal{C}^{+}, \mathcal{I}, \mathcal{F}, X, S, W^{a}, A_{0}^{+}, \mathcal{H}_{\mathrm{ad}}, \leq$

9.2. $X_{\Xi}, \gamma_{x}: \Xi \rightarrow \Xi, M^{\Xi}, M_{c}^{\Xi}$

9.4. $\Xi, \mathbf{M}_{c}$

9.7. $\epsilon_{\tau}, \tau * B$

9.8. $\kappa_{0}, \mathbf{e}_{0}$

9.9. $\mathbf{t}$

9.10. $\tilde{\mathbf{t}}, \tilde{\mathbf{e}}_{0}$

9.11. $\tilde{\mathbf{b}}, \mathbf{b}$

9.13. $A_{0}^{!}$

9.15. $\mathbf{M}_{\leq}, \mathbf{M}_{\geq}$

9.16. $\tilde{\mathbf{b}}^{\prime}, \mathbf{b}^{\prime}$

9.17. $B_{\leq}, \tilde{\pi}_{A, B}, B_{\geq}, \pi_{B, A}$ 
9.18. $\hat{\mathbf{b}}, \hat{\mathbf{b}}^{\prime}, \hat{B}_{\geq}, \hat{\pi}_{B, A}$

9.19. $\check{\mathbf{b}}, \check{\mathbf{b}}^{\prime}, \check{B}_{\leq}, \check{\pi}_{A, B}$

10.2. $\Phi$

\section{REFERENCES}

[BB] A. Bialynicki-Birula, Some theorems on actions of algebraic groups, Ann. of Math. 98 (1973), 480-497. MR 51:3186

[BS] W. M. Beynon and N. Spaltenstein, Green functions of finite Chevalley groups of type $E_{n}(n=6,7,8)$, J. of Algebra 88 (1984), 584-614. MR 85k:20136

[C] R. W. Carter, Finite groups of Lie type. Conjugacy classes and complex characters, John Wiley, 1985. MR 87d:20060

[DK] C. De Concini and V. Kac, Representations of quantum groups at roots of 1 , Operator Algebras, Unitary Representations, Enveloping Algebras and Invariant Theory (Colloque Dixmier), A. Connes et al. (eds.) Progress in Math., vol. 92, Birkhäuser, Boston, 1990. MR 92g:17012

[DLP] C. De Concini, G. Lusztig and C. Procesi, Homology of the zero-set of a nilpotent vector field on a flag manifold, J. Amer. Math. Soc. 1 (1988), 15-34. MR 89f: 14052

[D] D. Dokovic, Classification of nilpotent elements in simple exceptional real Lie algebras of inner type and description of their centralizers, J. of Alg. 112 (1988), 503-524. MR 89b: 17010

[F] W. Fulton, Intersection theory, 2nd ed., Springer Verlag, Berlin, Heidelberg, 1998. MR 99d:14003

[KL] D. Kazhdan and G. Lusztig, Proof of the Deligne-Langlands conjecture for Hecke algebras, Invent. Math. 87 (1987), 153-215. MR 88d:11121

[L1] G. Lusztig, Hecke algebras and Jantzen's generic decomposition patterns, Adv. in Math. 37 (1980), 121-164. MR 82b:20059

[L2] G. Lusztig, Intersection cohomology complexes on a reductive group, Invent. Math. $\mathbf{7 5}$ (1984), 205-272. MR 86d:20050

[L3] G. Lusztig, Cells in affine Weyl groups, Algebraic groups and related topics, Adv. Stud. Pure Math., vol. 6, North-Holland and Kinokuniya, Tokyo and Amsterdam, 1985, pp. 255287; II, J. Algebra 109 (1987), 536-548; III, J. Fac. Sci. Tokyo U. (IA) 34 (1987), 223-243; IV, J. Fac. Sci. Tokyo U. (IA) 36 (1989), 297-328. MR 87h:20074; MR 88m:20103a; MR 88m:20103b; MR 90k:20068

[L4] G. Lusztig, Periodic $W$-graphs, Represent. Theory (electronic) 1 (1997), 207-279. MR 99a:20042

[L5] G. Lusztig, Bases in equivariant K-theory, Represent. Theory (electronic) 2 (1998), 298369. MR 99i: 19005

[L6] G. Lusztig, Subregular nilpotent elements and bases in K-theory, Canad. J. Math. (1999).

[L7] G. Lusztig, Representation theory in characteristic $p$, Proceedings of Taniguchi Conference, Nara '98 (to appear).

[Se] J. Sekiguchi, Remarks on real nilpotent orbits of a symmetric pair, J. Math. Soc. Japan 39 (1987), 127-138. MR 88g:53053

[S] P. Slodowy, Simple algebraic groups and simple singularities, Lecture Notes in Math. 815, Springer Verlag, Berlin-Heidelberg-New York, 1980. MR 82g:14037

[Th] R. W. Thomason, Une formule de Lefschetz en K-théorie équivariante algébrique, Duke Math.J. 68 (1992), 447-462. MR 93m:19007

Institute for Advanced Study, Princeton, New Jersey 08540

Current address: Department of Mathematics, Massachusetts Institute of Technology, Cambridge, Massachusetts 02139

E-mail address: gyuri@math.mit.edu 